\title{
The role of Fluorine-18-Fluorodeoxyglucose positron emission tomography in staging and restaging of patients with osteosarcoma
}

\author{
Natale Quartuccio ${ }^{*}$, Giorgio Treglia2* ${ }^{2}$ Marco Salsano ${ }^{3}$, Maria Vittoria Mattoli $^{4}$, \\ Barbara Muoio ${ }^{5}$, Arnoldo Piccardo ${ }^{6}$, Egesta Lopci $^{7}$, Angelina Cistaro

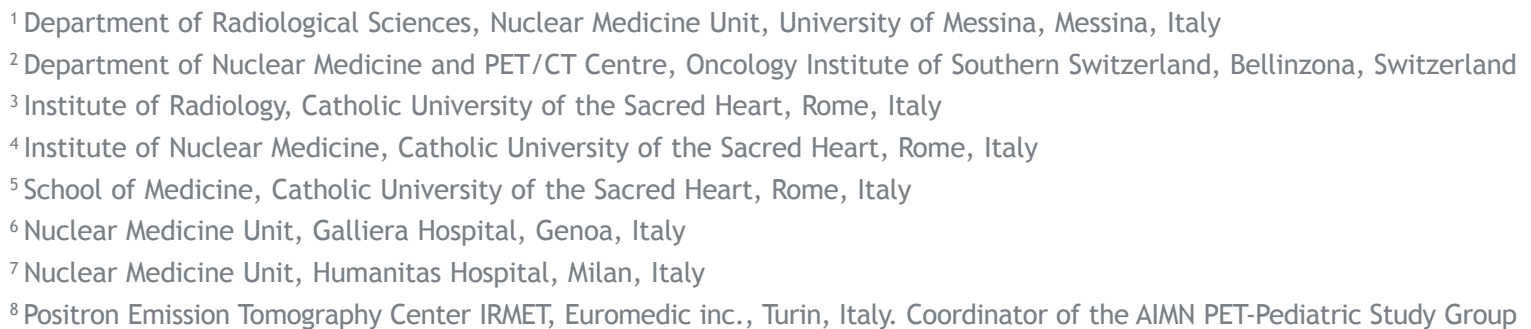

Radiol Oncol 2013; 47(2): 97-102.

Received 23 October 2012

Accepted 14 November 2012

Correspondence to: Angelina Cistaro, MD, Coordinator of the AIMN PET-Pediatric Study Group, Positron Emission Tomography Centre IRMET S.p.A., Via O. Vigliani 89, Turin 10136, Italy. Phone: +39 0113160158; Fax: +39 0113160828; E-mail: a.cistaro@irmet.com

*Natale Quartuccio and Giorgio Treglia equally contributed to this article sharing the first authorship

Disclosure: The authors have no conflicts of interest to disclose.

Background. The objective of this study is to systematically review the role of positron emission tomography (PET) and $\mathrm{PET} /$ computed tomography (PET/CT) with Fluorine-18-Fluorodeoxyglucose (FDG) in patients with osteosarcoma (OS). Methods. A comprehensive literature search of published studies through October 10 th, 2012 in PubMed/MEDLINE, Embase and Scopus databases regarding whole-body FDG-PET and FDG-PET/CT in patients with OS was performed. Results. We identified 13 studies including 289 patients with OS. With regard to the staging and restaging of OS, the diagnostic performance of FDG-PET and PET/CT seem to be high; FDG-PET and PET/CT seem to be superior to bone scintigraphy and conventional imaging methods in detecting bone metastases; conversely, spiral CT seems to be superior to FDG-PET in detecting pulmonary metastases from OS

Conclusions. Metabolic imaging may provide additional information in the evaluation of OS patients. The combination of FDG-PET or FDG-PET/CT with conventional imaging methods seems to be a valuable tool in the staging and restaging of $O S$ and may have a relevant impact on the treatment planning.

Key words: osteosarcoma; bone sarcoma; ${ }^{18 F-F l u o r o d e o x y g l u c o s e ; ~ P E T / C T ; ~ p o s i t r o n ~ e m i s s i o n ~ t o m o g r a p h y ~}$

\section{Introduction}

Osteosarcoma (OS) is the most common primary malignant bone tumour in children and adolescents, with a peak of incidence at the age of 15-19 years. ${ }^{1}$ OS is a tumour derived from primitive mesenchymal cells originating from bone and rarely from soft tissue. ${ }^{2}$ Although OS can occur in any bone, it is most common in the metaphyses of long bones: distal femur, proximal tibia, proximal humerus, and around the knee. ${ }^{3-5}$ OS has a high tendency to metastatic spread: $80 \%$ of all metastases arise in the lungs (20\% of them at initial diagnosis) but metastases can also develop in bone and rarely in lymph nodes. ${ }^{6-9}$ The 5-year survival rate for OS patients with metastases is $20 \%$ compared to $65 \%$ for patients with localised disease. ${ }^{10}$ 
Usually, the treatment scheme for patients with OS is comprised of pre-operative chemotherapy, surgical removal of all detectable tumour sites and/or local treatment, followed by post-operative chemotherapy. The prognosis for patients with metastatic disease or recurrent disease remains poor. $^{11,12}$ In order to correctly evaluate patients with OS in staging and restaging, a variety of diagnostic imaging modalities may be used, such as radiography, computed tomography (CT), magnetic resonance imaging (MRI), and bone scintigraphy. ${ }^{13}$ Fluorine-18-Fluorodeoxyglucose positron emission tomography (FDG-PET) has been successfully used to evaluate different malignant tumours ${ }^{14,15}$, such as musculoskeletal tumours. ${ }^{16}$ Tumour cells have a metabolic activity higher than normal cells and usually show an increased uptake of FDG, a glucose analogue. Like many other malignant tumours, OS have an increased rate of glycolysis, and consequently demonstrates an increased uptake of FDG. Standardised uptake value (SUV) can be used as semi-quantitative measure of the metabolic activity of a specific region of interest. ${ }^{17}$ Through the use of hybrid devices, integrating the high sensitivity of FDG-PET with the high spatial resolution of computed tomography (CT), a better diagnostic accuracy of PET/CT than PET and CT alone in detecting malignant tumours, such as OS, can be achieved. ${ }^{16-18}$ Several studies have shown the potential role of FDG-PET and PET/CT in the diagnosis of OS; however, a systematic review of published data in this field was lacking.

The purpose of this study is therefore to systematically review published data on the diagnostic performance of FDG-PET or PET/CT in patients with OS in order to assess the accuracy of these functional imaging methods in this setting.

\section{Materials and methods}

\section{Search strategy}

A comprehensive computer literature search of the PubMed/MEDLINE, Embase and Scopus databases was conducted in order to find relevant published articles on the diagnostic accuracy of FDG-PET and FDG-PET/CT in patients with osteosarcoma (OS). We used a search algorithm based on a combination of the terms: (a) "sarcoma" or "sarcomas " or "osteosarcoma" or "osteogenic sarcoma " or "bone sarcoma " or "bone sarcomas" or "pediatric tumors" or "pediatric tumours" or "pediatric sarcomas" or "pediatric sarcoma" or "childhood sarcomas" or "bone tumors" or "bone tumours" or "osseous sarcomas" or "skeletal sarcomas" or "skeletal sarcoma" or "musculoskeletal sarcomas" and (b) "positron emission tomography" or "Positron emission Tomography And Computed Tomography" or "PET". No beginning date limit was used; the search was updated until October $10^{\text {th }}$ 2012. Only articles in English language were selected. To expand our search, references of the retrieved articles were also screened for additional studies.

\section{Study selection}

Studies or subsets in studies investigating the diagnostic accuracy of FDG-PET or FDG-PET/CT in patients with OS were eligible for inclusion. Review articles, editorials or letters, comments, conference proceedings, articles not in the field of interest of this review, and case reports were excluded from this review. Only those studies or subsets in studies that satisfied all of the following criteria were included in the systematic review: (1) FDG-PET or FDG-PET/CT performed in patients with OS; (2) articles on the diagnostic accuracy of FDG-PET and FDG-PET/CT; (3) sample size of at least 10 patients with OS were included in the systematic review in order to select only the most relevant articles about the role of FDG-PET in osteosarcoma. Furthermore, this choice allowed reducing the publication bias. In fact, articles with a low number of patients usually report positive findings which further studies with a higher number of patients may exclude. When a possible overlap in patient data was found, the most complete article was included.

Four researchers (NQ, GT, MS and MVM) independently reviewed the titles and abstracts of the retrieved articles, applying the selection criteria mentioned above. Articles were rejected if they were clearly ineligible. The same four researchers then independently reviewed the full-text version of the remaining articles to determine their eligibility for the inclusion. Disagreements were resolved in a consensus meeting.

\section{Data extraction}

For each included study in the systematic review, information was collected concerning the basic study (authors, year of publication, journal, country of origin), device used (PET or PET/CT), and patient characteristics (number of patients undergoing PET or PET/CT, mean age, sex, and number of patients with OS). Finally, the main findings of all articles included in this review are shown in the results. 
TABLE 1. Characteristics of the studies included in the systematic review

\begin{tabular}{|c|c|c|c|c|c|c|c|c|c|}
\hline Authors & Year & Journal & Country & Study Design & Device used & $\begin{array}{c}\text { Number } \\
\text { of patients } \\
\text { performing } \\
\text { PET }\end{array}$ & $\begin{array}{c}\text { Mean } \\
\text { age } \\
\text { (years) }\end{array}$ & $\begin{array}{c}\text { Sex } \\
\text { (\%male) }\end{array}$ & $\begin{array}{l}\text { Number } \\
\text { of OS }\end{array}$ \\
\hline Garcia et al. ${ }^{23}$ & 1996 & J Nucl Med & USA & Prospective & PET & 48 & 40 & 50 & 18 \\
\hline Schulte et al. ${ }^{19}$ & 2000 & J Nucl Med & Germany & Prospective & PET & 202 & 28 & 63 & 44 \\
\hline Franzius et al. ${ }^{29}$ & 2001 & Ann Oncol & Germany & Retrospective & PET & 71 & 14 & 63 & 32 \\
\hline lagaru et al. ${ }^{30}$ & 2006 & $\begin{array}{l}\text { Nucl Med } \\
\text { Commun }\end{array}$ & USA & Retrospective & PET and PET/CT & 106 & 45 & 49 & 21 \\
\hline Kneisl et al. ${ }^{20}$ & 2006 & $\begin{array}{l}\text { Clin Orthop } \\
\text { Relat Res }\end{array}$ & USA & Retrospective & PET & 55 & NA & 51 & 38 \\
\hline Tateishi et al. ${ }^{25}$ & 2007 & Radiology & Japan & Retrospective & PET and PET/CT & 117 & 42 & 59 & 19 \\
\hline Völker et al. ${ }^{24}$ & 2007 & J Clin Oncol & Germany & Prospective & PET & 46 & 13 & 52 & 11 \\
\hline Charest et al. ${ }^{21}$ & 2009 & $\begin{array}{l}\text { Eur J Nucl } \\
\text { Med Mol } \\
\text { Imaging }\end{array}$ & Canada & Retrospective & $\mathrm{PET} / \mathrm{CT}$ & 212 & 47 & 52 & 24 \\
\hline London et al. ${ }^{27}$ & 2011 & $\begin{array}{l}\text { Pediatr } \\
\text { Radiol }\end{array}$ & Australia & Retrospective & $\mathrm{PET} / \mathrm{CT}$ & 41 & 13 & 63 & 20 \\
\hline $\begin{array}{l}\text { Bandopadhyaya } \\
\text { et al. }{ }^{28}\end{array}$ & 2012 & ISRN Oncol & India & Prospective & $\mathrm{PET} / \mathrm{CT}$ & 22 & 21 & 63 & 22 \\
\hline Cistaro et al. ${ }^{31}$ & 2012 & $\begin{array}{l}\text { Pediatr Blood } \\
\text { Cancer }\end{array}$ & Italy & Retrospective & $\mathrm{PET} / \mathrm{CT}$ & 18 & 14 & 61 & 11 \\
\hline Fuglø et al. ${ }^{21}$ & 2012 & $\begin{array}{l}\text { Eur J Nucl } \\
\text { Med Mol } \\
\text { Imaging }\end{array}$ & Denmark & Retrospective & $\mathrm{PET} / \mathrm{CT}$ & 30 & 30 & 47 & 14 \\
\hline
\end{tabular}

OS = osteosarcoma; NA = not available

\section{Results}

\section{Literature search}

The comprehensive computer literature search from the PubMed/MEDLINE, Embase, and Scopus databases revealed 28316 articles. Reviewing titles and abstracts, 13 articles comprising a total sample size of 289 patients with OS were selected applying the inclusion criteria mentioned above. ${ }^{19-31}$ These 13 studies were retrieved in their full-text version and included in this systematic review. No additional studies were found screening the references. The characteristics of the studies included are shown in Table 1.

\section{Literature data discussion}

\section{Initial assessment: grading and staging}

In 2000, Schulte et al. ${ }^{19}$ firstly demonstrated that FDG-PET, although never replacing biopsy, is a useful tool for estimating the biologic activity of skeletal lesions, including OS lesions. The authors evaluated the efficiency of FDG-PET in grading 202 patients with primary bone tumours, 44 of them with OS. FDG uptake was evaluated semi-quantitatively by determining the tumour-to-background ratio (T/B). Although sarcomas showed significantly higher $\mathrm{T} / \mathrm{B}$ values than benign lesions, in a few cases it was not possible to discriminate between benign and malignant lesions. Using a T/B cut-off level of 3.0 for malignancy, the sensitivity (SS), specificity (SP), accuracy, positive predictive value (PPV), and negative predictive (NPV) of FDG-PET were $93 \%, 66.7 \%, 81.7 \%, 78.7 \%$ and $87.9 \%$, respectively. No false negative findings occurred for patients with OS. ${ }^{16}$ In 2006, Kneisl and co-workers ${ }^{20}$ investigated the usefulness of FDG-PET in detecting occult non-pulmonary metastases at the initial work-up of 55 patients with bone sarcoma, 38 of them with OS. Only one of 38 OS patients (3\%) has been upstaged by FDG-PET. Thus, according to the authors, in consideration of the high cost of the study, the ability of PET scan to detect occult non-pulmonary metastases has a minimal influence in the clinical management of OS patients at initial work-up. ${ }^{20}$ In 2009, Charest et al. ${ }^{21}$ evaluated the diagnostic performance of FDG-PET/CT 
for detection of soft tissue and osseous sarcomas in 212 patients, including 24 OS. SS of FDG-PET/ CT for diagnosis of OS was $94.7 \%$, detecting 12/12 tumours in the initial assessment and 6/7 tumours in the restaging, with mean SUV of 8.9.21 Further confirming results were documented in 2012 by Fuglø et al. ${ }^{22}$ who retrospectively studied a group of 89 patients with high-grade soft tissue sarcomas (59) and bone sarcomas (30, 14 of which were OS) in the initial assessment. Limiting the analysis to the detection efficiency of FDG-PET/CT for distant metastases from bone sarcoma the SS, SP, accuracy, PPV and NPV were 88\%, 95\%, 95\%, 87\% and 98\%, respectively. In the lymph nodal based analysis FDG-PET/CT showed also high SS, SP, accuracy and NPV (100\%, 90\%, 91\% and 100\%, respectively) but a very low PPV (20\%) due to confounding inflammatory tissue with high glucose metabolism in most of the patients of the study. ${ }^{22}$

\section{Comparison with conventional imaging: staging and restaging}

In 1996, Garcia et al. ${ }^{23}$ compared the diagnostic accuracy of FDG-PET and 99mTc-sestaMIBI scintigraphy in 48 patients with suspected recurrent/residual musculoskeletal sarcomas, including 18 OS. FDG-PET appeared to be more sensitive to ${ }^{99 \mathrm{~m} T \mathrm{C}-}$ sestaMIBI scintigraphy in detecting active musculoskeletal sarcomas, with overall SS, SP, PPV and NPV of $98 \%, 90 \%, 98 \%$ and $90 \%$, respectively. ${ }^{23}$

Völker et al. ${ }^{24}$ also evaluated the impact of FDGPET for initial staging and therapy planning in 46 pediatric sarcoma patients, 11 of them with OS, demonstrating that the combination of FDG-PET with the conventional imaging is a valuable tool for the initial staging of OS and it has a relevant impact on therapy decisions. In fact, FDG-PET and conventional imaging reached the same efficiency in the detection of primary tumors (accuracy: 100\%). In addition FDG-PET showed a higher SS than conventional imaging regarding the detection of nodal metastases (95\% versus 25\%) and bone metastases. In particular, bone scintigraphy showed a higher number of false-negative lesions compared with FDG-PET. Instead CT was more reliable than FDGPET in depicting lung metastases, owing to their small size. Additionally combination of FDG-PET and conventional imaging changed the treatment planning in some cases. ${ }^{24}$ In the same year, Tateishi et al. ${ }^{25}$ demonstrated that the staging accuracy of combined PET/CT and conventional imaging is significantly higher than that of FDG-PET alone $(p<0.0001)$. The authors retrospectively compared the diagnostic accuracy of FDG-PET/CT, FDG-PET and conventional imaging (bone scintigraphy, chest radiography, diagnostic CT of the chest and abdomen and locoregional MRI) in detecting nodal and distant metastases in a group of soft-tissue and bone sarcomas (including 19 OS). The standard of reference was histology or adequate follow-up. FDGPET/CT showed to be superior to FDG-PET and conventional imaging in detecting nodal metastases. Similar results were documented comparing the ability of imaging modalities in detecting distant metastases. The authors conclude that the inclusion of FDG-PET/CT to the initial imaging workup yields to a more accurate preoperative staging of bone and soft-tissue sarcomas (mainly because of the more accurate M-staging) and this is important in determining the appropriate treatment. ${ }^{25}$

In 2009, Piperkova et al. ${ }^{26}$ retrospectively reviewed 93 patients with bone and soft tissue sarcomas (15 of them with OS) who underwent FDGPET/CT scan. The authors analyzed the results differentiating for FDG-PET alone, CT alone and combined FDG-PET/CT. For the initial staging, the combined FDG-PET/CT revealed the best performance, when compared with FDG-PET and CT alone, with a SS, SP, PPV, and NPV of $100 \%$. Also for the re-staging group, the combined FDG-PET/ CT revealed the best results with SS and SP of $100 \%$ and $95.9 \%$, respectively. The authors concluded that in bone and soft tissue sarcomas for the initial staging and re-staging FDG-PET/CT has higher accuracy than FDG-PET and CT alone. ${ }^{26}$

In 2011, London et al. ${ }^{27}$ evaluated the performance of FDG-PET/CT compared to the conventional imaging in detecting malignant lesions with particular attention to lung metastases and predicting a histological response to chemotherapy in 41 children with primary bone tumours (20 patients with OS). On a lesion based analysis, the SS, SP, and accuracy of FDG-PET/CT were $81.8 \%$, 97.5\%, and $95.9 \%$, respectively. In the lung lesion analysis, the SS, SP, and accuracy of FDG-PET/CT were $80.0 \%, 95.8 \%$, and $93.0 \%$, respectively. The authors concluded that FDG-PET/CT appears more accurate than the conventional imaging in detecting malignant lesions in childhood primary bone tumors, excluding lung lesions. ${ }^{27}$

In their prospective study in 2012, Bandopadhyaya et al..$^{28}$ evaluated 22 biopsy proved OS patients undergoing FDG-PET/CT and 99mTcDimercaptosuccinic acid (99mTc-DMSA) whole body scintigraphy and compared the detection efficiency of the two imaging modalities. In detecting the primary lesion ${ }^{99 m}$ Tc-DMSA scintigraphy 
showed the same SS (100\%) of FDG-PET but lower SS in depicting lung metastases probably because the limited resolution of gamma camera respect to $\mathrm{PET} / \mathrm{CT}$, which instead reported a SS of $100 \% .^{28}$

\section{Comparison with conventional Imaging: evaluation of pulmonary lesions}

Franzius et al. ${ }^{29}$ compared FDG-PET and spiral thoracic $\mathrm{CT}$ in detecting pulmonary metastases from malignant primary osseous tumors in 71 patients, including 32 patients with OS. In OS patients, FDGPET revealed a SS, SP, and accuracy of $50 \%, 100 \%$, and $92 \%$, respectively. In all 71 patients (32 with OS and 39 with Ewing sarcoma), spiral thoracic CT revealed a SS, SP, and accuracy of $75 \%, 100 \%$, and $94 \%$, respectively. The authors concluded that spiral CT seemed to be superior compared to FDGPET in detecting pulmonary metastases from malignant primary bone tumors, although a positive FDG-PET result can be used to confirm abnormalities seen on thoracic CT scans as neoplastic. ${ }^{29}$ In 2006, Iagaru et al..$^{30}$ published a retrospective study of 106 patients with the histological diagnosis of osseous and soft tissue sarcomas (21 of them with OS), assessing the ability of FDG-PET and FDG$\mathrm{PET} / \mathrm{CT}$ versus chest $\mathrm{CT}$ in detecting pulmonary metastases. Overall, concordant PET and CT detection of pulmonary metastases was noted in 27 patients $(67.5 \%)$. For all the patients, the SS and SP for FDG-PET were $68.3 \%$ and $98.4 \%$, respectively. CT had a SS of $95.1 \%$ and SP of $92.3 \%$. The authors demonstrated that $\mathrm{CT}$ of the chest was more sensitive than PET in detecting pulmonary metastases from OS; a significant portion of pulmonary nodules $>1 \mathrm{~cm}$ on CT are PET-negative; sub-centimeter CT lesions should not be considered false positive if inactive on PET; a negative PET scan in the presence of suspicious CT findings in the chest cannot reliably exclude pulmonary metastases from osseous and soft tissue sarcomas. ${ }^{30}$ In 2012 Cistaro et al..$^{31}$ studied 18 patients, 11 of which with OS, who had undergone FDG-PET/CT scan. They firstly attempted to find a SUVmax cut-off value helpful in discriminating the nature of the pulmonary nodules in pediatric bone sarcoma patients. They showed that a SUVmax threshold $>1.09$ was highly suggestive of malignancy when the nodule diameter was $>6 \mathrm{~mm}$. No significant advantage was found in the semi-quantitative analysis (SUV max and $\mathrm{SUV}_{\text {ratio }}$ ) for the assessment of lesions below 6 $\mathrm{mm}$. In the entire group of patients ${ }^{18} \mathrm{~F}-\mathrm{FDG}-\mathrm{PET} /$ CT had a SS of $90.3 \%$, a SP of $87.5 \%$, a PPV of $87.5 \%$, and a NPV of $90.3 \%$ and an accuracy of $88.9 \% .{ }^{31}$

\section{Conclusions and general remarks}

From this systematic review on the role of FDGPET and FDG-PET/CT in patients with OS, we are led to conclude that:

1) The combined metabolic and morphological information of FDG-PET/CT imaging allows a high diagnostic accuracy for the detection of OS; FDGPET/CT is significantly more accurate than FDGPET alone and improves staging and restaging in patients with OS.

2) With regard to the staging and restaging of OS, the SS, SP, and accuracy of FDG-PET and PET/ CT seem to be high; FDG-PET and PET/CT seem to be superior to bone scintigraphy and conventional imaging methods in detecting bone metastases; conversely, spiral CT seems to be superior to FDGPET in detecting pulmonary metastases from OS. A combination of FDG-PET/CT with conventional imaging methods is a valuable tool for staging and restaging of OS and may have a relevant impact on the treatment planning.

3) Most of the articles included in this systematic review evaluated the diagnostic accuracy of FDGPET or PET/CT in mixed populations with different types of sarcomas, including some patients with OS. Further large prospective and multicenter studies evaluating the diagnostic accuracy of FDGPET/CT in patients with OS are needed.

\section{References}

1. Mirabello L, Troisi RJ, Savage SA. Osteosarcoma incidence and survival rates from 1973 to 2004: data from the Surveillance, Epidemiology, and End Results Program. Cancer 2009; 115: 1531-43.

2. Ritter J, Bielack SS. Osteosarcoma. Ann Oncol 2010; 21: 320-5.

3. Arndt CA, Crist WM. Common musculoskeletal tumors of childhood and adolescence. N Engl J Med 1999; 341: 342-52.

4. Bielack SS, Kempf-Bielack B, Delling G, Exner GU, Flege S, Helmke K, et al. Prognostic factors in high-grade osteosarcoma of the extremities or trunk: an analysis of 1,702 patients treated on neoadjuvant cooperative osteosarcoma study group protocols. J Clin Oncol 2002; 20: 776-90.

5. Bielack $S$, Jürgens $H$, Jundt $G$, Kevric $M$, Kühne $T$, Reichardt $P$, et al. Osteosarcoma: the COSS experience. Cancer Treat Res 2009; 152: 289-308.

6. Bacci G, Rocca M, Salone M, Balladelli A, Ferrari S, Palmerini E, et al. High grade osteosarcoma of the extremities with lung metastases at presentation: treatment with neoadjuvant chemotherapy and simultaneous resection of primary and metastatic lesions. J Surg Oncol 2008; 98: 415-20.

7. Bielack SS, Carrle D, Hardes J, Schuck A, Paulussen M. Bone tumors in adolescents and young adults. Curr Treat Options Oncol 2008; 9: 67-80.

8. Messerschmitt PJ, Garcia RM, Abdul-Karim FW, Greenfield EM, Getty PJ. Osteosarcoma. J Am Acad Orthop Surg 2009; 17: 515-27.

9. Kager L, Zoubek A, Pötschger U, Kastner U, Flege S, Kempf-Bielack B, et al. Cooperative German-Austrian-Swiss Osteosarcoma Study Group. Primary metastatic osteosarcoma: presentation and outcome of patients treated on neoadjuvant Cooperative Osteosarcoma Study Group protocols. I Clin Oncol 2003; 21: 2011-8. 
10. Posthuma De Boer J, Witlox MA, Kaspers GJ, van Royen BJ. Molecular alterations as target for therapy in metastatic osteosarcoma: a review of literature. Clin Exp Metastasis 2011; 28: 493-503.

11. Kim DH, Kim SY, Lee HJ, Song BS, Kim DH, Cho JB, et al. Assessment of Chemotherapy Response Using FDG-PET in Pediatric Bone Tumors: A Single Institution Experience. Cancer Res Treat 2011; 43: 170-5.

12. Wuisman P, Enneking WF. Prognosis for patients who have osteosarcoma with skip metastasis. J Bone Joint Surg Am 1990; 72: 60-8.

13. Xu J, Shen J, Ding Y, Shen HY, Zeng ZP, Ma RF, et al. The clinical value of combined use of MR imaging and multi-slice spiral CT in limb salvage surgery for orthopaedic oncology patients: initial experience in nine patients. Radiol Oncol 2012; 46: 189-97.

14. Chen J, Zhao Y, Li X, Sun $P$, Wang $M$, Wang $R$, et al. Imaging primary prostate cancer with 11C-Choline PET-CT: relation to tumour stage, Gleason score and biomarkers of biologic aggressiveness. Radiol Oncol 2012; 46: 179-88.

15. Kim JS, Jeong YJ, Sohn MH, Jeong HJ, Lim ST, Kim DW, et al. Usefulness of F-18 FDG PET/CT in subcutaneous panniculitis-like T cell lymphoma: disease extent and treatment response evaluation. Radiol Oncol 2012; 46: 279-83.

16. Lakkaraju A, Patel CN, Bradley KM, Scarsbrook AF. PET/CT in primary musculoskeletal tumours: a step forward. Eur Radiol 2010; 20: 2959-72.

17. Treglia G, Cason E, Fagioli G. Recent applications of nuclear medicine in diagnostics (first part). Ital J Med. 2010; 4: 84-91

18. Treglia G, Salsano M, Stefanelli A, Mattoli MV, Giordano A, Bonomo L Diagnostic accuracy of ${ }^{18}$ F-FDG-PET and PET/CT in patients with Ewing sarcoma family tumours: a systematic review and a meta-analysis. Skeletal Radiol 2012; 41: 249-56.

19. Schulte M, Brecht-Krauss D, Heymer B, Guhlmann A, Hartwig E, Sarkar MR, et al. Grading of tumors and tumorlike lesions of bone: evaluation by FDG PET. J Nucl Med 2000; 41: 1695-701.

20. Kneisl JS, Patt JC, Johnson JC, Zuger JH. Is PET useful in detecting occult nonpulmonary metastases in pediatric bone sarcomas? Clin Orthop Relat Res 2006; 450: 101-4.

21. Charest M, Hickeson M, Lisbona R, Novales-Diaz JA, Derbekyan V, Turcotte RE. FDG PET/CT imaging in primary osseous and soft tissue sarcomas: a retrospective review of 212 cases. Eur J Nucl Med Mol Imaging 2009; 36: 1944-51.

22. Fuglø HM, Jørgensen SM, Loft A, Hovgaard D, Petersen MM. The diagnostic and prognostic value of 18F-FDG PET/CT in the initial assessment of highgrade bone and soft-tissue sarcoma. A retrospective study of 89 patients. Eur J Nucl Med Mol Imaging 2012; 39: 1416-24.

23. Garcia R, Kim EE, Wong FC, Korkmaz M, Wong WH, Yang DJ, et al. Comparison of fluorine-18-FDG PET and technetium-99m-MIBI SPECT in evaluation of musculoskeletal sarcomas. J Nucl Med 1996; 37: 1476-9.

24. Völker T, Denecke T, Steffen I, Misch D, Schönberger S, Plotkin M, et al. Positron emission tomography for staging of pediatric sarcoma patients: results of a prospective multicenter trial. J Clin Oncol 2007; 25: 5435-41.

25. Tateishi U, Yamaguchi U, Seki K, Terauchi T, Arai Y, Kim EE. Bone and softtissue sarcoma: preoperative staging with fluorine 18 fluorodeoxyglucose PET/CT and conventional imaging. Radiology 2007; 245: 839-47.

26. Piperkova E, Mikhaeil M, Mousavi A, Libes R, Viejo-Rullan F, Lin H. Impact of $\mathrm{PET}$ and $\mathrm{CT}$ in PET/CT studies for staging and evaluating treatment response in bone and soft tissue sarcomas. Clin Nucl Med 2009; 34: 146-50.

27. London K Stege C, Cross S, Onikul E, Graf N, Kaspers G, et al. 18F-FDG PET/ $\mathrm{CT}$ compared to conventional imaging modalities in pediatric primary bone tumors. Pediatr Radiol 2012; 42: 418-30.

28. Bandopadhyaya GP, Gupta P, Singh A, Shukla J, Rastogi S, Kumar R, et al. (99m)Tc-DMSA (V) in evaluation of osteosarcoma: comparative studies with (18)F-FDG PET/CT in detection of primary and malignant lesions. ISRN Oncol. 2012; 2012: 371830.

29. Franzius C, Daldrup-Link HE, Sciuk J, Rummeny EJ, Bielack $\mathrm{S}$, Jürgens $\mathrm{H}$, et al. FDG-PET for detection of pulmonary metastases from malignant primary bone tumors: comparison with spiral CT. Ann Oncol 2001; 12: 479-86.

30. lagaru A, Chawla S, Menendez L, Conti PS. 18F-FDG PET and PET/CT for detection of pulmonary metastases from musculoskeletal sarcomas. NuCl Med Commun 2006; 27: 795-802.

31. Cistaro A, Lopci E, Gastaldo L, Fania P, Brach Del Prever A, et al. The role of (18) F-FDG PET/CT in the metabolic characterization of lung nodules in pediatric patients with bone sarcoma. Pediatr Blood Cancer. 2012; 59: 1206-10. 


\title{
Brain and whole-body FDG-PET in diagnosis, treatment monitoring and long-term follow-up of primary CNS lymphoma
}

\author{
Sofiane Maza', Ralph Buchert², Winfried Brenner², Dieter Ludwig Munz², Eckhard Thiel ${ }^{3}$, \\ Agnieszka Korfel ${ }^{3}$, Philipp Kiewe ${ }^{3}$ \\ ${ }^{1}$ Department of Nuclear Medicine, Vivantes MVZ Spandau, Berlin, Germany \\ 2 Department of Nuclear Medicine, ${ }^{3}$ Department of Hematology, Charité-Universitätsmedizin, Berlin, Germany
}

Radiol Oncol 2013; 47(2): 103-110.

Received 18 June 2012

Accepted 29 December 2012

Correspondence to: Dr. Sofiane Maza, MD, PhD, Vivantes MVZ Spandau, Neue Bergstrasse 6, 13585 Berlin, Germany. Phone: +49 301301 31883; Fax: +49 301302 9131883; E-mail: sofiane.maza@gmail.com

Disclosure: No potential conflicts of interest were disclosed

Background. Positron emission tomography (PET) with F-18-labeled fluorodeoxyglucose (FDG) provides remarkable accuracy in detection, treatment monitoring and follow-up of systemic malignant lymphoma. Its value in the management of patients with primary central nervous system lymphoma (PCNSL) is less clear.

Patients and methods. In a prospective trial, 42 FDG-PET examinations were performed in ten immunocompetent patients with newly diagnosed or recurrent PCNSL before and repeatedly during and after the treatment. Brain and whole body FDG-PET were compared to brain MRI and extra-cerebral CT, respectively.

Results. Before the treatment, 6 of 10 patients had congruent findings on FDG-PET and MRI of the brain. Three patients had lesions on brain MRI, not detected by FDG-PET. One patient had additional FDG-PET positive lesions inconspicuous in MRI. The follow-up suggested FDG-PET to be false positive in these lesions. After the treatment, brain PET was in agreement with MRI in 6 of 8 patients. In the remaining 2 patients there were persistent lesions in brain MRI whereas FDG-uptake was reduced to normal values. In the long-term follow-up of 5 patients (63-169 weeks), 3 patients retained normal in both PET and MRI. In 2 patients a new focal pathologic FDG-uptake was detected 69 and 52 weeks after the end of the treatment. In one of these patients, recurrence was confirmed by MRI not until 9 weeks after PET. Conclusions. Brain FDG-PET may contribute valuable information for the management of PCNSL, particularly in the assessment of the treatment response. Integration of FDG-PET into prospective interventional trials is warranted to investigate prognostic and therapeutic implications.

Key words: PET-CT; primary central nervous system lymphoma; response assessment; imaging

\section{Introduction}

Magnetic resonance imaging (MRI) is the standard diagnostic modality when primary central nervous system lymphoma (PCNSL) is suspected, often showing characteristic radio-morphological features such as lesion location adjacent to cerebrospinal fluid (CSF) space, strong and homogenous contrast-enhancement, moderate oedema and absence of necrosis. MRI of the brain is commonly used for the evaluation of the treatment response, however, with several limitations. Contrast-enhancement re- flecting blood-brain barrier (BBB) disruption may be caused not only by the tumour, but also by surgical intervention, radiotherapy or chemotherapy. Misinterpretation as tumour residuum in these latter cases results in the unnecessary treatment which might cause late neurotoxicity. In follow-up, the differentiation of malignant lesions from other lesions like inflammatory processes or scars can sometimes be difficult in MRI. ${ }^{1}$

F-18-labelled fluorodeoxyglucose (FDG) positron emission tomography (PET) has demonstrated remarkable sensitivity in the detection 
of systemic non-Hodgkin's lymphoma (NHL). Furthermore, it has been shown to provide high accuracy in the differentiation between cerebral lymphomas and either high-grade gliomas or infectious lesions in patients with acquired immunodeficiency syndrome (AIDS). ${ }^{1-3}$ As FDG-uptake in the brain relates to the rate of tissue glucose metabolism, FDG-PET is not limited to the assessment of BBB integrity but provides functional information on tumor viability. ${ }^{4}$

A few studies have evaluated FDG-PET in immunocompetent PCNSL patients. ${ }^{5-13}$ However, no study has systematically addressed the value of repeated FDG-PET in both treatment-monitoring and long-term follow-up in PCNSL in the same patients. Therefore, the aim of the present prospective study was to evaluate potential diagnostic benefits of FDG-PET in a sample of immunocompetent PCNSL patients in the assessment of disease extent prior to the treatment as well as in treatment monitoring and in long-term follow-up.

\section{Patients and methods}

The research has complied with all relevant national regulations and institutional policies and has been approved by the authors' institutional review board or equivalent committee.

\section{Patients}

The study included ten immunocompetent patients (5 females, median age at inclusion 54.5 years) with PCNSL who were scheduled for the treatment at our institution. Seven patients had newly diagnosed PCNSL with a histological proof of high-grade B-cell lymphoma. Systemic lymphoma was excluded by computed tomography (CT) and bone marrow biopsy. Three patients had recurrent PCNSL with a histological proof at first diagnosis. HIV infection was excluded in all patients.

All 7 patients with newly diagnosed PCNSL received chemotherapy with high-dose methotrexate (HDMTX) for up to a total of 6 cycles. Of 3 patients treated for recurrent disease, one patient (No. 3) received HDMTX plus ifosfamide, and 2 patients (No. 9 and 10) were treated with Y-90-labeled (90Y) ibritumomab tiuxetan.

\section{Imaging protocol}

Several imaging sessions were scheduled for each patient, each session including both FDG-PET (brain and whole-body) and MRI (brain only). Initially, a 'baseline session' was performed before the treatment initiation, followed by 'therapy monitoring sessions' after 3 HDMTX cycles (in the 7 patients with newly diagnosed PCNSL), and 4-6 weeks after the completion of the treatment. Thereafter, 'follow-up sessions' were performed every 3-4 months.

\section{FDG-PET imaging}

Brain FDG-PET scans were started 60 min after intravenous injection of about $370 \mathrm{MBq}$ FDG with an acquisition time of 20 minutes, followed by a whole body scan including 6-8 bed positions. An ECAT EXACT system (30 sessions) and later a Biograph 16 system (12 sessions) was used (Siemens, Erlangen, Germany). Transversal images were generated using the standard reconstruction algorithm of the system software. The attenuation correction was based on either a Ge-68 transmission scan (ECAT EXACT) or a low-dose CT (Biograph).

Reconstructed images were first analysed visually. Suspect areas of focally increased FDG uptake were further analysed by region of interest (ROI) analysis. In the brain, circular ROIs of 6.4 $\mathrm{mm}$ diameter were placed manually in the lesion, centred at the hottest voxel. The maximum standardized uptake value (SUV max, maximum activity concentration in the ROI / (injected dose / body weight)) was used for semi-quantitative analysis. In addition, an FDG uptake ratio was computed by dividing mean SUV in the ROI by mean SUV in the (manually placed) mirror ROI in the other hemisphere (normal tissue). This scaling procedure reduces variability by elimination of global effects and, thus, improves the power for the detection of local pathological changes.

In order to determine the normal range of the FDG uptake ratio in the present study, the uptake ratio analysis was performed for healthy cortical gray matter, healthy white matter, and healthy thalamus in each brain FDG PET scan in each patient. Univariate analyses of variance with uptake ratio as dependent variable, tissue type (gray, white, thalamus) as fixed factor, and time (delay in days to baseline scan of the same patient) as covariate did not detect any significant effect. Therefore, all uptake ratios were grouped together, resulting in mean \pm 1 standard deviation of the sample $=1.010 \pm 0.045$ (range $0.913-1.149$ ). Defining the upper threshold of the normal range by mean +3 standard deviations resulted in a threshold of 1.15 for the uptake ratio to be pathologically increased. 


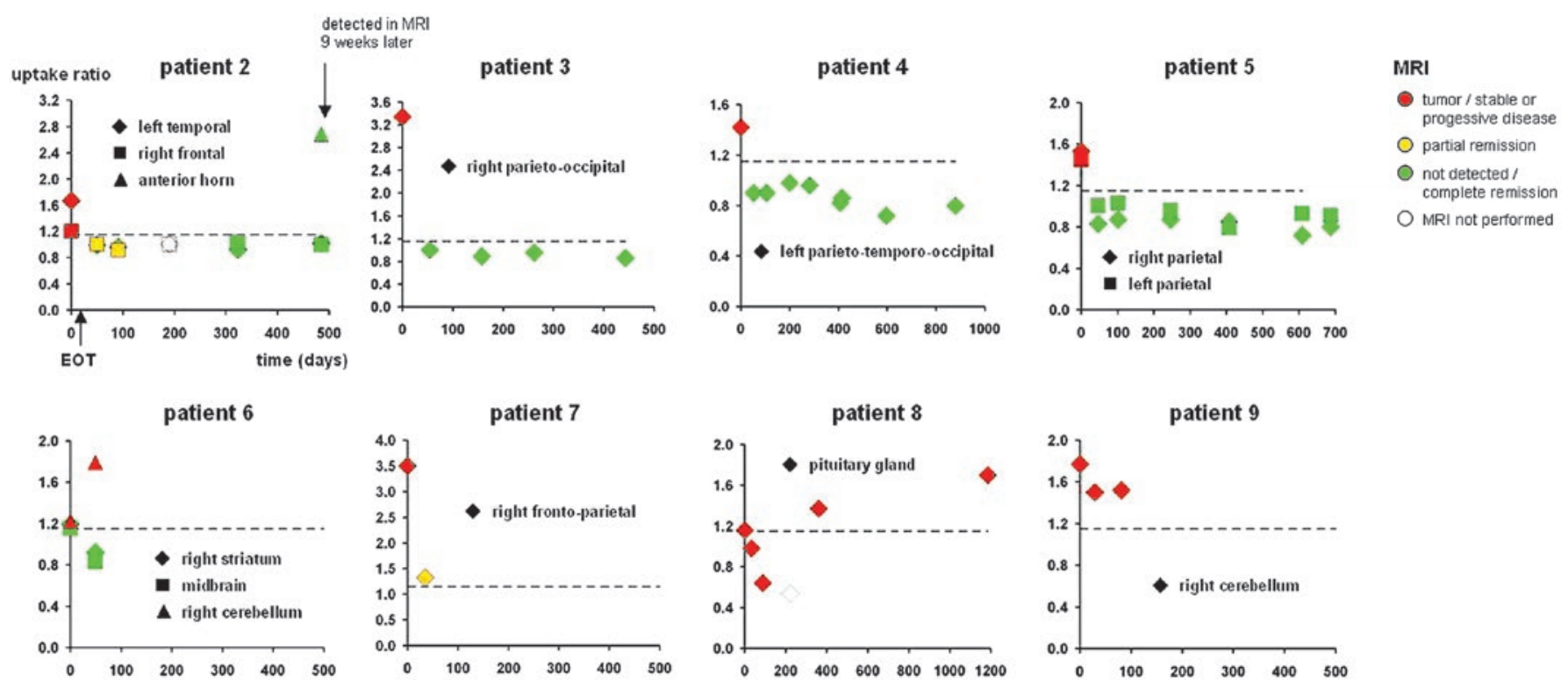

FIGURE 1. Summary of brain PET and MRI findings in all patients with at least one follow-up session. Patients 1 and 10 did not show any suspect lesion in the FDG-PET of the brain.

\section{MR imaging}

All MRI studies were carried out using a standardized protocol including sagittal T1-weighted spin echo (slice thickness $5 \mathrm{~mm}$, inter-slice gap $0.5 \mathrm{~mm}$ ), axial T2-weighted fast spin echo, coronal FLAIR and axial T1-weighted spin echo before and after Gd-DTPA injection. Additional sagittal and coronal T1-weighted spin echo images were acquired when contrast enhancement was observed on axial slices. The response on MRI was evaluated according to International PCNSL Group (IPCG) criteria. ${ }^{14}$ For response evaluation, patients had to be off-steroids to differentiate between the effect of steroids and chemotherapy.

FDG-PET and MRI were interpreted independently by an experienced nuclear medicine physician and an experienced radiologist, respectively. Both readers were blinded to clinical data.

\section{Results}

\section{Baseline}

The results at baseline are summarized in Table 1 and Figure 1. In the 7 newly diagnosed patients, baseline MRI was performed pre-biopsy in 3 and post-biopsy in 4 patients. Of patients with prebiopsy MRI (No. 2, 4, and 5), two patients had stereotactic biopsy, the other patient (No. 5) had open resection of one lesion. Of patients with postbiopsy MRI (No. 1, 6, 7, and 8), one patient (No. 1) had stereotactic biopsy, one patient (No. 8) open biopsy, and 2 patients (No. 6 and 7) had open lesion resection.

FDG-PET was performed after MRI and postbiopsy in all patients. Seven patients were on corticosteroids at the time of the PET examination.

Number and location of cerebral lesions at baseline was in agreement between PET and MRI in 6 of 10 patients (No. 3, 4, 5, 7, 8, 9; the right frontal MRI lesion in patient No. 5 had been resected prior to FDG-PET and, therefore, was excluded from the analysis (Table 1). Disagreement between PET and MRI at baseline occurred in 4 of 10 patients (No. 1, 2, 6, 10). In 3 of these patients (No. 1, 2, 10) FDGPET of the brain showed less lesions than MRI. In 2 of these 3 patients (No. 1 and 10) FDG-PET was rated entirely normal, whereas MRI detected a single contrast enhancing lesion suspicious of PCNSL in the left thalamus (No. 1) and multiple lesions in the cerebellum (No. 10), respectively. In the $3^{\text {rd }}$ of these patients (No. 2) FDG-PET was negative in a MRI lesion in the left cerebellum, but positive in the 2 further MRI lesions of this patient. In the remaining patient with disagreement between FDGPET and MRI at baseline (No. 6), FDG-PET showed 2 lesions (right striatum, midbrain) which had not been detected by MRI. A lesion in the right cerebellum of this patient was detected by both modalities. Maximal SUV value of brain lesions in the PET ranged between 3.6 and 12.5 (median 5.3), the uptake ratio ranged between 1.2 and 3.5 (median 1.5).

Whole-body FDG-PET detected extra-cerebral lesions in 2 of 10 patients (No. 3 and 4). In patient 
TABLE 1. Imaging findings at baseline

\begin{tabular}{|c|c|c|c|c|c|c|c|c|c|c|c|c|}
\hline ż & 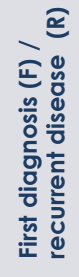 & 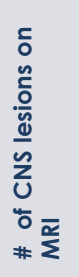 & 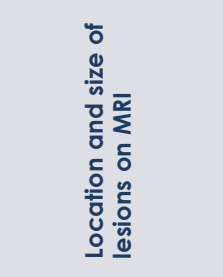 & 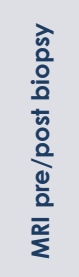 & $\begin{array}{l}\text { बे } \\
\frac{0}{0} \\
\frac{0}{0} \\
\frac{0}{0} \\
\frac{t}{0} \\
\frac{0}{x}\end{array}$ & 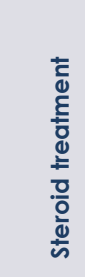 & 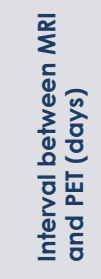 & 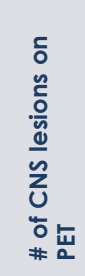 & 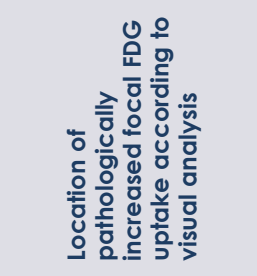 & 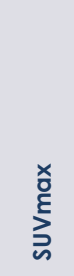 & $\begin{array}{l}\frac{0}{\bar{t}} \\
\frac{0}{0} \\
\frac{0}{0} \\
\frac{0}{3}\end{array}$ & 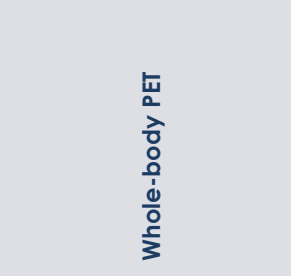 \\
\hline \multirow[t]{2}{*}{2} & $\mathrm{~F}$ & 3 & $\begin{array}{l}\text { left temporal } \\
(2.2 \times 1.25 \mathrm{~cm})\end{array}$ & pre & $\begin{array}{l}\text { Stereotactic } \\
\text { (left } \\
\text { temporal } \\
\text { lesion) }\end{array}$ & yes & 14 & 2 & left temporal & 4.1 & 1.7 & normal \\
\hline & & & right frontal & & & & & & right frontal & 4.2 & 1.2 & \\
\hline 4 & $\mathrm{~F}$ & 1 & $\begin{array}{l}\text { left parieto- } \\
\text { temporo- } \\
\text { occipital } \\
(3 \times 3 \mathrm{~cm})\end{array}$ & pre & Stereotactic & yes & 14 & 1 & $\begin{array}{l}\text { left parieto- } \\
\text { temporo-occipital }\end{array}$ & 4.2 & 1.4 & $\begin{array}{l}\text { Pathologically } \\
\text { increased FDG-uptake } \\
\text { in left kidney/adrenal } \\
\text { gland (stable in follow- } \\
\text { up PET) }\end{array}$ \\
\hline 5 & $\mathrm{~F}$ & 3 & $\begin{array}{l}\text { right frontal } \\
(3 \times 1.8 \mathrm{~cm})\end{array}$ & pre & $\begin{array}{l}\text { Open } \\
\text { resection of } \\
\text { right frontal } \\
\text { lesion }\end{array}$ & yes & 7 & 2 & $\begin{array}{l}\text { low FDG-Uptake } \\
\text { after open } \\
\text { resection right } \\
\text { frontal }\end{array}$ & & & normal \\
\hline \multirow{2}{*}{6} & & & & & & & & & right striatum & 6.0 & 1.2 & \\
\hline & & & & & & & & & midbrain & 4.6 & 1.2 & \\
\hline 7 & $\mathrm{~F}$ & 1 & $\begin{array}{l}\text { right fronto- } \\
\text { parietal } \\
(4.5 \times 2.5 \mathrm{~cm})\end{array}$ & post & $\begin{array}{l}\text { Open } \\
\text { resection }\end{array}$ & yes & 21 & 1 & right fronto-parietal & 3.6 & 3.5 & normal \\
\hline 8 & $\mathrm{~F}$ & 1 & $\begin{array}{l}\text { pituitary gland } \\
(1 \times 1 \mathrm{~cm})\end{array}$ & post & Open biopsy & no & 10 & 1 & pituitary gland & 8.7 & 1.2 & normal \\
\hline 9 & $\mathrm{R}$ & 1 & $\begin{array}{l}\text { right cerebellum } \\
(3 \times 2 \mathrm{~cm})\end{array}$ & NA & No biopsy & no & 7 & 1 & right cerebellum & 10.0 & 1.8 & normal \\
\hline 10 & $R$ & mult & $\begin{array}{l}\text { left cerebellum } \\
(3.75 \times 2 \mathrm{~cm})\end{array}$ & NA & No biopsy & yes & 23 & 0 & & & & normal \\
\hline
\end{tabular}

CNS = central nervous system; MRI = magnetic resonance imaging; SUVmax = maximal standard uptake value; TU = tumour; PET = positron emission tomography; NA = not applicable; FDG = F-18-fluorodeoxyglucose; mult = multiple 
Initial imaging
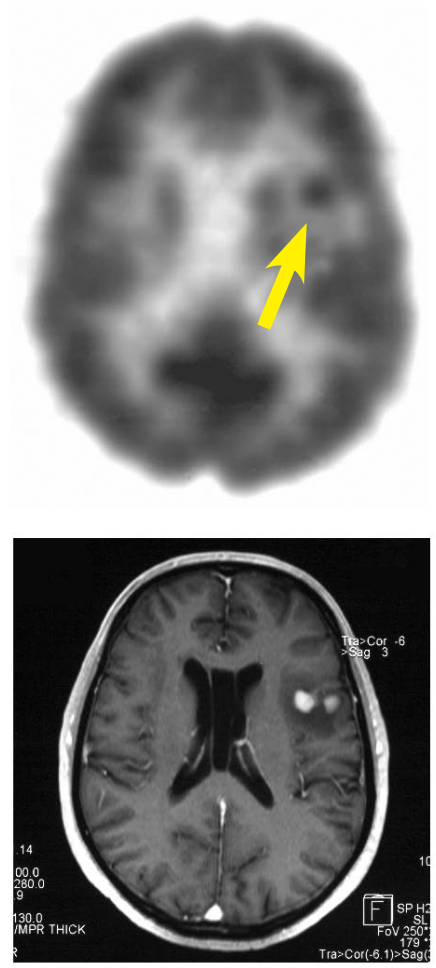

After treatment
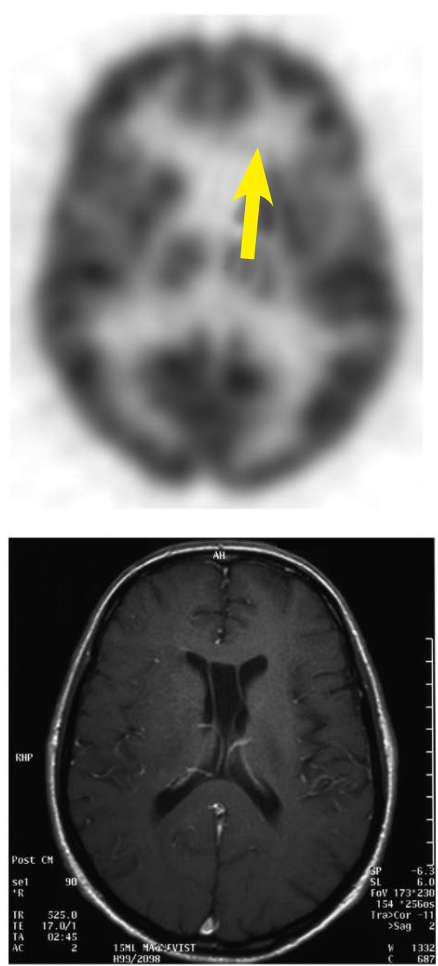

Follow-up
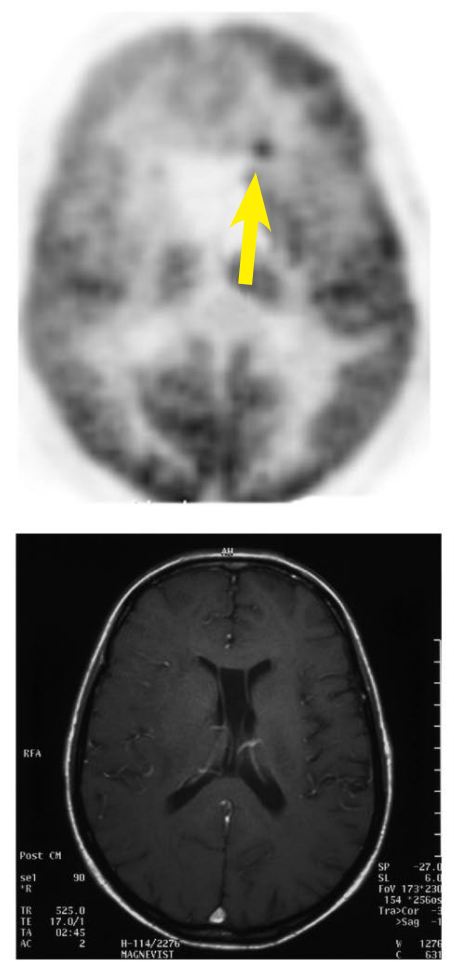

Follow-up +9 weeks

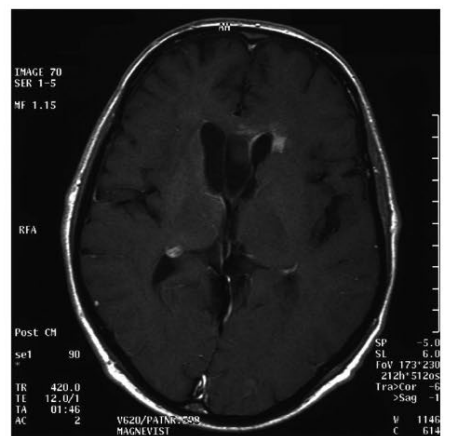

FIGURE 2. Baseline PET and MRI of patient No. 2 shows left temporal lesion. No pathological FDG-uptake was observed after therapy but a new lesion in the anterior horn was seen on PET 69 weeks later (SUV=10.5). MRI at this time point was still categorized as complete remission. A contrast-enhancing lesion appeared on MRI 9 weeks thereafter.

No. 3, who had recurrent PCNSL, pathologic FDGuptake was detected in the lung, mediastinal lymph nodes and thoracic wall. These findings were confirmed as asymptomatic systemic lymphoma manifestations by subsequent CT. In patient No. 4, pathologically increased FDG-uptake was found in the left kidney without a correlative on CT. The lesion was confirmed in the follow-up PETs.

\section{Therapy monitoring}

The results of the therapy monitoring are summarized in Table 2 and Figure 1. Imaging for therapy monitoring was performed after the completion of the therapy in all cases. An additional imaging session during 3 cycles of HDMTX was performed in 1 patient (No. 3). All patients were off steroids at the time of monitoring sessions.

The evaluation of the therapy response by MRI was performed in 9 patients. Of these, complete response $(\mathrm{CR})$ was found in 3 patients, partial response (PR) in 2, stable disease / minimal response
(SD/MR) in 2, and progressive disease (PD) in 2 patients.

Therapy monitoring by FDG-PET was performed in 8 patients. FDG-PET was in agreement with MRI in 6 of these: CR in MRI and normal FDG-PET in 3 patients (No. 3, 4, 5), PR in MRI and decreased FDG uptake compared to baseline (but still elevated) in 1 patient (No. 7), stable disease in MRI and persistent pathologic FDG uptake in 1 patient (No. 9), and PD in MRI and increase of pathologic FDG uptake in 1 lesion in PET in 1 patient (No. 6). There was a disagreement between MRI and FDG-PET in the remaining 2 patients. FDGPET was rated normal in both of these patients, whereas MRI was rated as PR (No. 2) or even SD/ MR (No. 8). The first of these patients (No. 2) did not show recurrence of the lesions detected at baseline during the follow-up of 69 weeks, suggesting that the normal FDG-PET finding after the therapy was true negative, despite of the disease progression with a novel lesion in another part of the brain at this late time point (frontal horn, Figure 2). In 
TABLE 2. Evaluation of treatment response

\begin{tabular}{llll}
\hline No. & Treatment & MRI & PET \\
\hline 1 & Ix HDMTX & ND (death) & ND (death) \\
2 & 6x HDMTX & PR & No pathologic uptake \\
3 & $\begin{array}{l}\text { 6x HDMTX }+ \\
\text { Ifosfamide }\end{array}$ & $\begin{array}{l}\text { CR (cerebral) after } 3 \text { cycles, CR } \\
\text { (thoracic) after } 6 \text { cycles }\end{array}$ & $\begin{array}{l}\text { No pathologic uptake in the brain after } 3 \text { cycles, No } \\
\text { pathologic uptake in the chest after } 6 \text { cycles }\end{array}$ \\
4 & $5 x$ HDMTX & CR & No pathologic uptake \\
5 & 6x HDMTX & CR & No pathologic uptake \\
6 & $2 x$ HDMTX & PD & Increase of pathologic uptake in 1 of 3 lesions \\
7 & $2 x$ HDMTX & PR & Pathologic uptake reduced \\
8 & 6x HDMTX & SD/MR & No pathologic uptake \\
9 & $\begin{array}{l}\text { Ibritumomab } \\
\text { tiuxetan }\end{array}$ & SD & Persistant pathologic uptake \\
10 & $\begin{array}{l}\text { Ibritumomab } \\
\text { tiuxetan }\end{array}$ & PD & ND \\
\hline
\end{tabular}

$\mathrm{PET}=$ positron emission tomography; $\mathrm{HDMTX}=$ high-dose methotrexate; $\mathrm{ND}=$ not done; $\mathrm{PR}=$ partial response; $\mathrm{CR}=\mathrm{complete}$ response; $\mathrm{PD}=$ progressive disease; $S D=$ stable disease; $M R=$ minimal response

the second of these patients (No. 8), recurrence was observed in FDG-PET 52 weeks after the treatment.

\section{Follow-up}

Results of the follow-up are summarized in Table 3 and Figure 1. A long-term follow-up by both MRI and FDG-PET was performed in 5 patients (No. 2, 3, $4,5,8)$. Median time of the follow-up was 98 weeks (range 63-169). In 3 of these patients (No. 3, 4, 5) both MRI and FDG-PET showed a complete remission during the whole period of the follow-up. In 1 patient (No. 2), a new pathologic FDG uptake (SUV $\max =10.5$, uptake ratio $=2.7$ ) was detected by PET in the anterior horn after 69 weeks. MRI revealed tumour $\mathrm{r}$ relapse not until 9 weeks after positive PET (Figure 2). In another patient (No. 8), a new pathologic FDG uptake (SUV max $=8.6$; uptake ratio $=1.4$ ) was seen on PET after 52 weeks along with documented stable disease on MRI.

\section{Discussion}

First results on FDG-PET in PCNSL were reported by Rosenfeld et al. who, investigating 10 patients, found FDG-uptake in PCNSL lesions to be similar to that of anaplastic gliomas. ${ }^{10}$ In a series of seven patients evaluated at the time of initial diagnosis, Palmedo et al. found a good correlation between gadolinium enhancement in MRI and focal FDGuptake on PET with merely one lesion missed by PET and another lesion visible only on the FLAIR
MRI sequence. ${ }^{9}$ In the present study, there was an agreement between PET and MRI before therapy in 6 of 10 patients. In 3 of the remaining 4 patients, FDG-PET showed fewer lesions suspicious of PCNSL than contrast-enhanced MRI. Two of these 3 patients were rated normal (no lesion) in FDG PET. This discrepancy might be explained by initiation of corticosteroid therapy between MRI and PET. Corticosteroid-induced reduction of FDG-uptake has been reported in cerebral lymphoma. ${ }^{5}$ In one patient, the residual contrast enhancement of a cerebellar lesion on MRI might have been caused by postoperative BBB dysfunction. In this case the negative FDG-PET might indicate the absence of vital tumour after the resection. In 1 patient, PET showed brain lesions of the increased FDG uptake that were not detected in MRI. However, neither specific neurological symptoms nor follow-up supported the presence of lymphoma in these lesions. Most likely the increased tracer uptake was due to benign postoperative processes in these cases. In summary, FDG-PET might provide valuable information in addition to MRI with respect to disease extent at initial imaging before therapy in most patients.

In the present study, FDG-PET included a whole-body scan in addition to the brain scan, in contrast to most previous studies. Whole-body FDG-PET was in agreement with CT in all patients newly diagnosed with PCNSL. The increased FDG-uptake in the left kidney in one newly diagnosed patient was without correlate in $\mathrm{CT}$, also in retrospective inspection of the $\mathrm{CT}$, and did not 
change in follow-up PET, and, therefore, most likely was not malignant. Pathological extra cerebral FDG-uptake was found in one patient with PCNSL relapse, and was subsequently confirmed as systemic lymphoma by CT. Based on this finding, the treatment was changed in this patient: systemic chemotherapy was initiated instead of whole-body immunoradiotherapy as had been planned prior to whole-body PET. This important finding might have been missed in clinical routine patient care, since abdominal or thoracic CT is not routinely performed in relapsed PCNSL patients. A previous case report by Karantanis et al. also suggests that whole-body PET might be superior to CT in the detection of extracranial disease in PCNSL. ${ }^{12}$ In a retrospective study, Mohile et al. evaluated the contribution of whole-body FDG-PET in staging and restaging of PCNSL patients. ${ }^{6}$ These authors found systemic disease in $7 \%$ of patients with suspected PCNSL, which would have been missed with conventional CT. This rate was even higher (27\%) in patients with CNS relapse. Thus, wholebody FDG-PET might result in change of the treatment strategy in a considerable fraction of patients.

Response evaluation can be difficult in PCNSL, since the residual contrast enhancement caused by the treatment-induced disruption of the BBB is not infrequent. The differentiation between $C R$ and $\mathrm{PR}$, which is of paramount importance for the decision about the further treatment, is often particularly difficult. Standardized criteria for the evaluation of the treatment response in PCNSL include the category of 'unconfirmed complete remission' $(\mathrm{CRu})$ for patients needing corticosteroids despite disappearance of all gadolinium-enhancing lesions on MRI and patients with a small but persistent contrast enhancement after biopsy or focal haemorrhage. ${ }^{14}$ FDG-PET is expected to be particularly useful in these cases. In the present longitudinal prospective study, FDG PET was performed for the response evaluation as well as during the follow-up in the same patients. FDG-PET provided different information about the therapy response than MRI in 2 of 8 patients. In both patients, FDGPET indicated CR despite of the persistent contrast enhancement in MRI. There was no recurrence of the initial lesion during the follow up of 69 weeks in one of these patients, suggesting a true negative FDG-PET, despite of disease progression with novel lesions in other parts of the brain. In the second of these patients, FDG-PET indicated a complete remission whereas MRI findings were categorized as stable disease / minor response. The recurrence was observed in FDG-PET only 52 weeks after the
TABLE 3. Long-term follow-up

\begin{tabular}{lll}
\hline No. & MRI & PET \\
\hline 1 & ND (death) & ND (death) \\
2 & $\begin{array}{l}\text { CR at 69 weeks after } \\
\text { treatment. Relapse } \\
\text { detected 78 weeks } \\
\text { after treatment }\end{array}$ & $\begin{array}{l}\text { new pathologic } \\
\text { uptake 69 weeks after } \\
\text { treatment }\end{array}$ \\
3 & CR & normal \\
4 & CR & normal \\
5 & CR & normal \\
6 & ND & ND \\
7 & ND & ND \\
8 & PR & Recurrence of \\
9 & ND & pathologic FDG 52 \\
10 & ND & ND after treatment \\
\hline
\end{tabular}

$\mathrm{PET}=$ positron emission tomography; $\mathrm{ND}=$ not done; $\mathrm{PR}=$ partial response; $\mathrm{CR}=$ complete response

treatment. These findings are in agreement with the results of Palmedo et al., who reported truly negative PET findings (confirmed by follow-up) despite the persistence of lesions on MRI in three of six patients. ${ }^{9} \mathrm{~A}$ response to initial chemotherapy as assessed by MRI has consistently been associated with the prolonged survival in PCNSL. ${ }^{15,16}$ The present findings suggest that FDG-PET might improve the response assessment and, therefore, might provide improved prognostic power compared to MRI. However, further studies with larger patient samples are required to test this hypothesis.

In the long-term follow-up of one patient, relapse was detected by FDG-PET but not by MRI at this time point. Relapse was confirmed by MRI 9 weeks later. However, while FDG-PET might have the potential to detect relapse earlier than MRI, this benefit might not be relevant in clinical routine, because making use of this benefit would require very short intervals between follow-up imaging which cannot be justified on a routine base due to the radiation exposure. In most cases, the relapse is detected by the occurrence of new neurologic symptoms rather than by routine imaging.

The main limitations of the present study are relatively small sample size, heterogeneity among the patients with respect to various factors (newly diagnosed versus recurrence, treatment strategy), a time interval of more than 2 weeks between MRI and PET at baseline in 3 out of 10 patients, corticosteroid treatment at the time of baseline FDG 
PET in 7 out of 10 patients, use of two different PET scanners with slightly different spatial resolution, and incomplete follow-up in some patients. Nevertheless, the data suggest that FDG-PET may contribute valuable information in PCNSL. Wholebody FDG-PET might be useful for staging prior to therapy. FDG-PET of the brain might be useful for the evaluation of the treatment response, particularly in case of mild residual contrast-enhancement in MRI.

\section{References}

1. Kimura N, Yamamoto Y, Kameyama R, Hatakeyama T, Kawai N, Nishiyama Y. Diagnostic value of kinetic analysis using dynamic 18F-FDG-PET in patients with malignant primary brain tumor. Nucl Med Commun 2009; 30: 602-9.

2. Heald $A E$, Hoffman JM, Bartlett JA, Waskin HA. Differentiation of central nervous system lesions in AIDS patients using positron emission tomography (PET). Int J STD AIDS 1996; 7: 337-46.

3. Pierce MA, Johnson MD, Maciunas RJ, Murray MJ, Allen GS, Harbison MA, et al. Evaluating contrast-enhancing brain lesions in patients with AIDS by using positron emission tomography. Ann Intern Med 1995; 123: 594-8.

4. Di Chiro G. Positron emission tomography using [18F] fluorodeoxyglucose in brain tumors. A powerful diagnostic and prognostic tool. Invest Radiol 1987; 22: 360-71.

5. Kuwabara $Y$, Ichiya $Y$, Otsuka M, Miyake $Y$, Gunasekera R, Hasuo K, et al. High [18F]FDG uptake in primary cerebral lymphoma: a PET study. J Comput Assist Tomogr 1988;12: 47-8.

6. Mohile NA, Deangelis LM, Abrey LE. The utility of body FDG PET in staging primary central nervous system lymphoma. Neuro Oncol 2008; 10: 223-8.

7. Mohile NA, Deangelis LM, Abrey LE. Utility of brain FDG-PET in primary CNS lymphoma. Clin Adv Hematol Oncol 2008; 6: 818-20, 840.

8. Kawai N, Okubo S, Miyake K, Maeda Y, Yamamoto Y, Nishiyama Y, et al. Use of PET in the diagnosis of primary CNS lymphoma in patients with atypical MR findings. Ann Nucl Med 2010; 24: 335-43.

9. Palmedo $\mathrm{H}$, Urbach $\mathrm{H}$, Bender H, Schlegel U, Schmidt-Wolf IG, Matthies A, et al. FDG-PET in immunocompetent patients with primary central nervous system lymphoma: correlation with MRI and clinical follow-up. Eur J NuC Med Mol Imaging 2006; 33: 164-8.

10. Rosenfeld SS, Hoffman JM, Coleman RE, Glantz MJ, Hanson MW, Schold SC. Studies of primary central nervous system lymphoma with fluorine18-fluorodeoxyglucose positron emission tomography. J Nucl Med 1992; 33: $532-6$.

11. Karantanis D, O'Eill B P, Subramaniam RM, Witte RJ, Mullan BP, Nathan MA et al. 18F-FDG PET/CT in primary central nervous system lymphoma in HIVnegative patients. Nucl Med Commun 2007; 28: 834-41.

12. Karantanis D, O'Neill BP, Subramaniam RM, Peller PJ, Witte RJ, Mullan BP, et al. Contribution of F-18 FDG PET-CT in the detection of systemic spread of primary central nervous system lymphoma. Clin Nucl Med 2007; 32: 271-4.

13. Nishiyama $Y$, Yamamoto $Y$, Monden T, Sasakawa $Y$, Kawai N, Satoh K, et al. Diagnostic value of kinetic analysis using dynamic FDG PET in immunocompetent patients with primary CNS lymphoma. Eur J Nucl Med Mol Imaging 2007; 34: 78-86.

14. Abrey LE, Batchelor TT, Ferreri AJ, Gospodarowicz M, Pulczynski EJ, Zucca $E$, et al. Report of an international workshop to standardize baseline evaluation and response criteria for primary CNS lymphoma. J Clin Oncol 2005; 23: 5034-43.

15. Kiewe $P$, Fischer $L$, Martus $P$, Thiel E, Korfel A. Primary central nervous system lymphoma: monocenter, long-term, intent-to-treat analysis. Cancer 2008; 112: 1812-20.

16. Korfel A, Martus P, Nowrousian MR, Hossfeld DK, Kirchen H, Brucher J, et al. Response to chemotherapy and treating institution predict survival in primary central nervous system lymphoma. Br J Haematol 2005; 128: 177-83. 


\title{
Poor outcome of comprehensive therapy in a case of laryngeal synovial sarcoma
}

\author{
Yang-Yang Bao1, Quin-Ying Wang¹, Shui-Hong Zhou¹, Kui Zhao², Ling-Xiang Ruan³, \\ Hong-Tian Yao

\begin{abstract}
${ }^{1}$ Department of Otolaryngology, ${ }^{2}$ Department of PET/CT centre, ${ }^{3}$ Department of Radiology, ${ }^{4}$ Department of Pathology, The First Affiliated Hospital, College of Medicine, Zejiang University, Hangzhou, Zhejiang, China
\end{abstract}

Radiol Oncol 2013; 47(2): 111-118.

Received 3 August 2012

Accepted 10 February 2013

Correspondence to: Dr Shui-Hong Zhou, Ph.D, The First Affiliated Hospital, College of Medicine, Zhejiang University, Quingchun road 79, Hangzhou; Zhejiang, China, 310003. Phone: 86-13868060120; Fax: 86-571-87236895; E-mail: zhouyunzhoush@163.com

Disclosure: No potential conflicts of interest were disclosed.

Background. Synovial sarcoma is common in the extremities. Our search revealed only 17 cases of synovial sarcoma of the larynx in the English-language literature.

Case report. We report an additional case of a 37-year-old man with primary laryngeal synovial sarcoma who underwent positron emission tomography/computed tomography (PET/CT) following the treatment. Although the patient received comprehensive therapy including surgery, radiotherapy, repeated chemotherapies, and targeted therapies, he had an unfavourable outcome and died of distant metastases.

Conclusions. In synovial sarcoma of the larynx, PET/CT can detect recurrence and metastasis. PET/CT can also predict the treatment effect in patients with synovial sarcoma.

Key words: synovial sarcoma; larynx; PET/CT; follow up

\section{Introduction}

Synovial sarcoma is common in the extremities. ${ }^{1}$ Only $3-9 \%$ of all cases of synovial sarcoma occur in the head and neck region, and the least frequent site of occurrence is the larynx.,3 Our search revealed only 17 cases of synovial sarcoma of the larynx in the English-language literature. ${ }^{2-18}$

Since few cases of laryngeal synovial sarcoma have been reported, its histogenesis, natural history, optimal treatment strategy, and long-term prognosis are unknown. Most primary synovial sarcomas of the head and neck metastasise to the lungs; among these, only a few cases were reported to have cervical metastases. ${ }^{19}$ The local recurrence rate of synovial sarcoma is $8-60 \%$. Tumours usually recur within 2 years of the initial therapy. ${ }^{20}$ The diagnostic workup for recurrence or metastasis of synovial sarcoma involves conventional imaging, including computed tomography (CT) and magnetic resonance imaging (MRI). ${ }^{21}$ During the past decade, ${ }^{18} \mathrm{~F}$-fluorodeoxyglucose $\left({ }^{18} \mathrm{FDG}\right)$ positron emission tomography (PET)/CT has become an adjunct tool to conventional imaging in the staging and follow-up of sarcoma. ${ }^{22} \mathrm{~A}$ few reports have described the use of PET/CT for synovial sarco$\mathrm{ma}^{1,20,23}$, and no report has presented the PET/CT features of laryngeal synovial sarcoma.

In the majority of cases, the surgical excision with a wide margin is the first treatment choice. The effect of chemotherapy or radiotherapy is controversial. ${ }^{24-26}$ We report a case of a patient with primary laryngeal synovial sarcoma who underwent PET/CT following treatment. Although the patient received comprehensive therapy including surgery, radiotherapy, repeated chemotherapies, and targeted therapies, he had an unfavourable outcome and died of whole-body metastasis in November 2011. 


\section{Case report}

On 4 June 2008, a 37-year-old man was referred to our department due to a 1-month history of sore throat and blood in the phlegm. He also complained of dysphagia. He denied hoarseness and respiratory dyspnoea. His medical history was unremarkable. Laryngoscopy showed a $2 \times 2.5 \mathrm{~cm}$ wine-coloured tumour in the right aryepiglottic fold that involved the inner wall of the right piriform sinus. The surface was covered with blood clots. The motion of the bilateral vocal cords and the remainder of the larynx appeared normal. Cervical lymphadenopathy was absent. CT revealed a $3.5 \times 2.2 \mathrm{~cm}$ irregular soft-tissue mass in the right aryepiglottic fold extending to the right piriform sinus, with low to moderate heterogeneous enhancement after the injection of contrast medium. No enlarged node
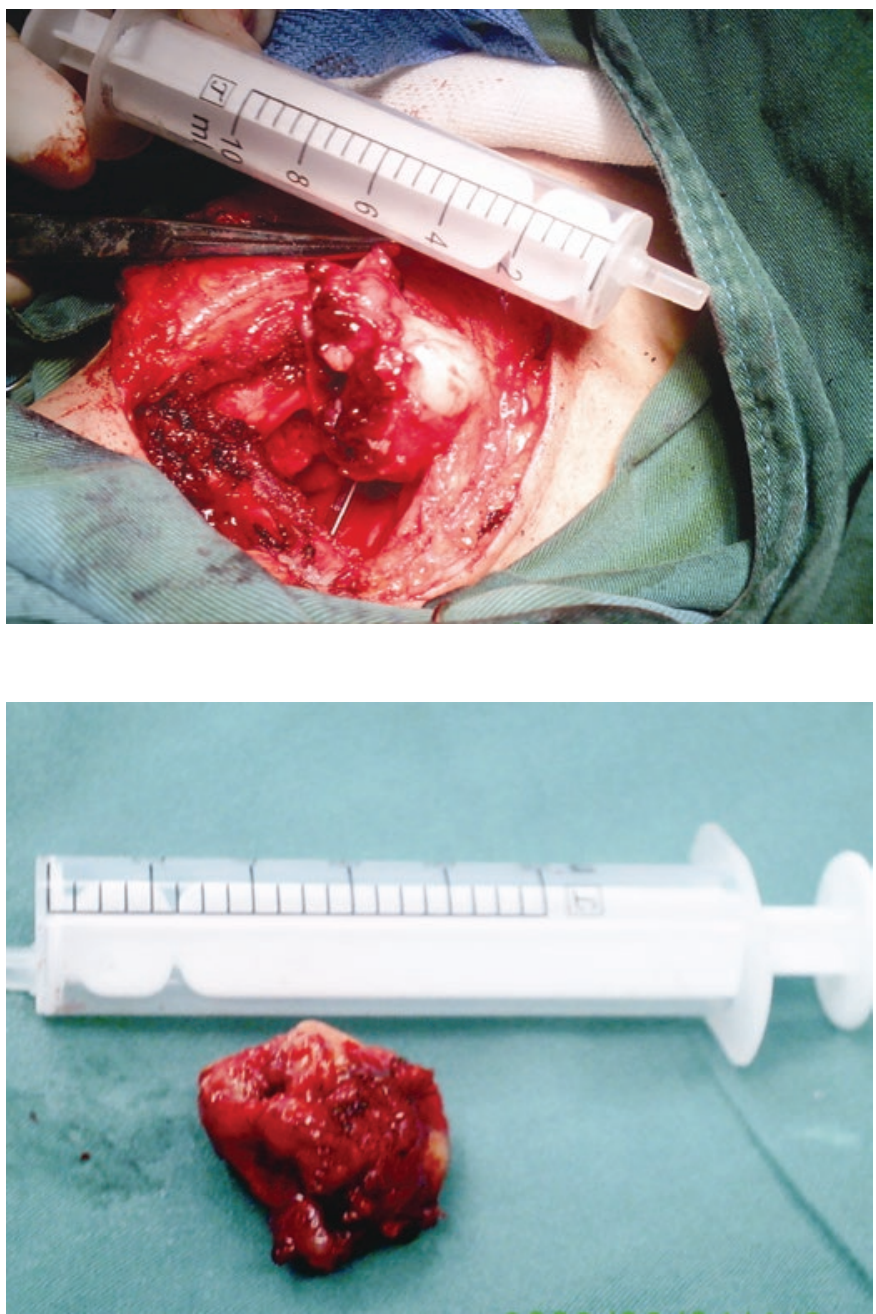

FIGURE 1. (a) The mass was tan-red and irregular and had an ulcer covered by blood clots and a fleshy cut surface. (b) Surgical sample. was present. Under general anaesthesia (a tracheotomy was performed), biopsy was performed by suspension laryngoscopy. The frozen section results suggested that the tumour was a spindle cell tumour. The tumour was removed completely via lateral cervical incision and partial laryngectomy. The laryngeal function was preserved. During the operation, we found that the tumour was located in the right supraglottic area, with a pedicle in the right aryepiglottic fold extending to the right piriform sinus. Mass was tan-red and irregular and had an ulcer covered by blood clots and a fleshy cut surface (Figure 1).

The postoperative pathological results showed that the tumour consisted of small, uniform spindle cells invading the surrounding muscles. The immunohistochemical examination for vimentin and CD99 was positive. Epithelial membrane antigen was focal positive. These results led to a diagnosis of monophasic synovial sarcoma. The resection margins were tumour free (Figure 2).

Postoperative radiotherapy was given; the total dose was 66 Gy. On 26 October 2009, MRI of the head and neck revealed a $1 \times 2 \mathrm{~cm}$ mass in the region of the right hypopharynx and laryngeal inlet. On 4 November 2009, ${ }^{18}$ FDG-PET/CT demonstrated high FDG uptake coincident with MRI findings (standardised uptake value $[S U V]_{\max }=4.1$ ) and no distant metastases (Figure 3). A partial laryngopharyngectomy was performed. After surgery, the patient was followed regularly at 2-month intervals with CT or MRI examination of the head and neck.

In August 2010, CT of the head and neck revealed a $3.6 \times 2.6 \mathrm{~cm}$ mass in the right submaxillary region. Further CT of the lungs revealed bilateral lung metastases. PET/CT revealed high FDG uptake in the right submaxillary lymph node $\left(\mathrm{SUV}_{\max }=3.2\right)$, right oropharynx $\left(\mathrm{SUV}_{\max }=5.4\right)$, and multiple nodules in the bilateral lungs $\left(\mathrm{SUV}_{\max }=4.6\right.$; Figure 4$)$. These results suggested local recurrence and cervical lymph node and lung metastases. The patient received chemotherapy and concurrent targeted treatment comprising adriamycin $\left(40 \mathrm{mg} / \mathrm{m}^{2}\right.$, days 1-2), ifosfamide (2 $\mathrm{g} / \mathrm{m}^{2}$, days $\left.1-4\right)$, dacarbazine (300 mg/m², days 1-4), and anti-epidermal growth factor receptor monoclonal antibody (nimotuzum$\mathrm{ab}, 200 \mathrm{mg} / \mathrm{m}^{2}$, days 1-4). The patient underwent three cycles of chemotherapy at 3-week intervals. On 18 November 2010, the first-line therapy package was completed. The cervical mass was treated with intratumoural injection of recombinant adenovirus p53 agent injection once a week for 4 weeks and another four cycles of nimotuzumab 
TABLE 1 English-Literature review of synovial sarcomas of larynx

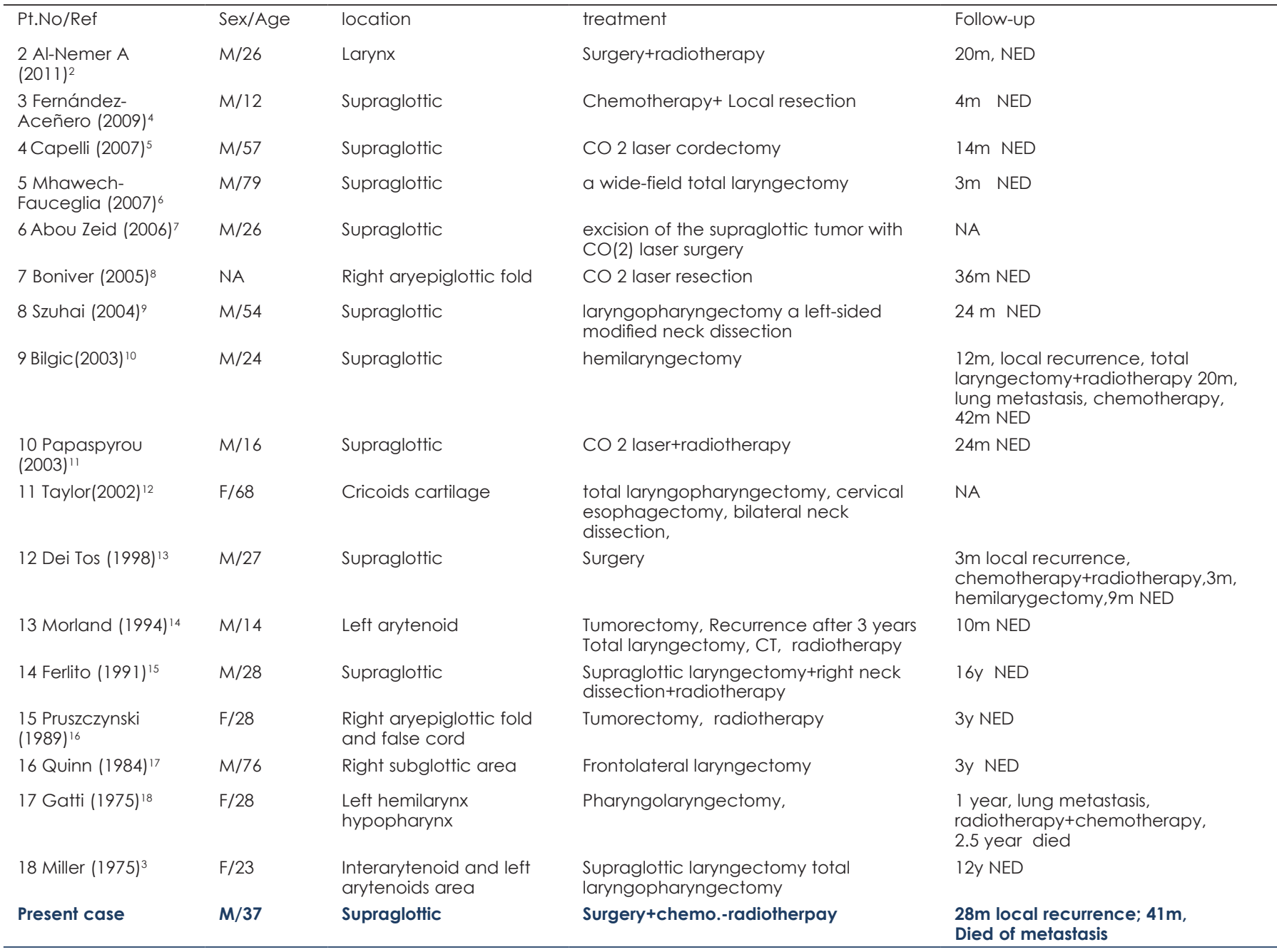

Ref $=$ number of reference; NED = no evidence of disease; NA = not available

(200 mg) at 1-week intervals. CT demonstrated no regression of the metastatic cervical lymph node and lung lesions. In February 2011, the patient underwent additional chemotherapy comprising docetaxel (110 mg, day 1), cisplatin (40 mg, days 1-3), and rh-endostatin (Endostar) (15 mg, days 1-14) every 21 days for up to five cycles. The lesions were not controlled. In July 2011, the chemotherapy was changed to cetuximab (600 mg, day 1) every week for up to three cycles. CT of the lungs and MRI of the head and neck showed that the lesions were larger. On 22 September 2011, PET/CT revealed a $7.5 \times 9 \mathrm{~cm}$ mass in the right submaxillary region with high FDG uptake $\left(\mathrm{SUV}_{\max }=5.2\right)$ involving the right parotid gland, right tongue base, right mandible, jugular vein, carotid artery, and surround- ing muscles, and multiple nodules in the bilateral lungs (largest $=6.68 \mathrm{~cm}$ in diameter; $\mathrm{SUV}_{\max }=6.02$; Figure 5). The cervical mass was treated by local cryoablation, but did not regress. In 11 November 2011, the patient died of brain metastases.

\section{Discussion}

Only 3-9\% of all cases of synovial sarcoma occur in the head and neck. The least frequent site of occurrence is the larynx. To our knowledge, only 18 cases have been reported (including the current case) (Table 1). ${ }^{2-18}$ The reported cases included 13 males, three females, and one case in which the patient's sex was not reported..$^{2-18}$ The male to female 

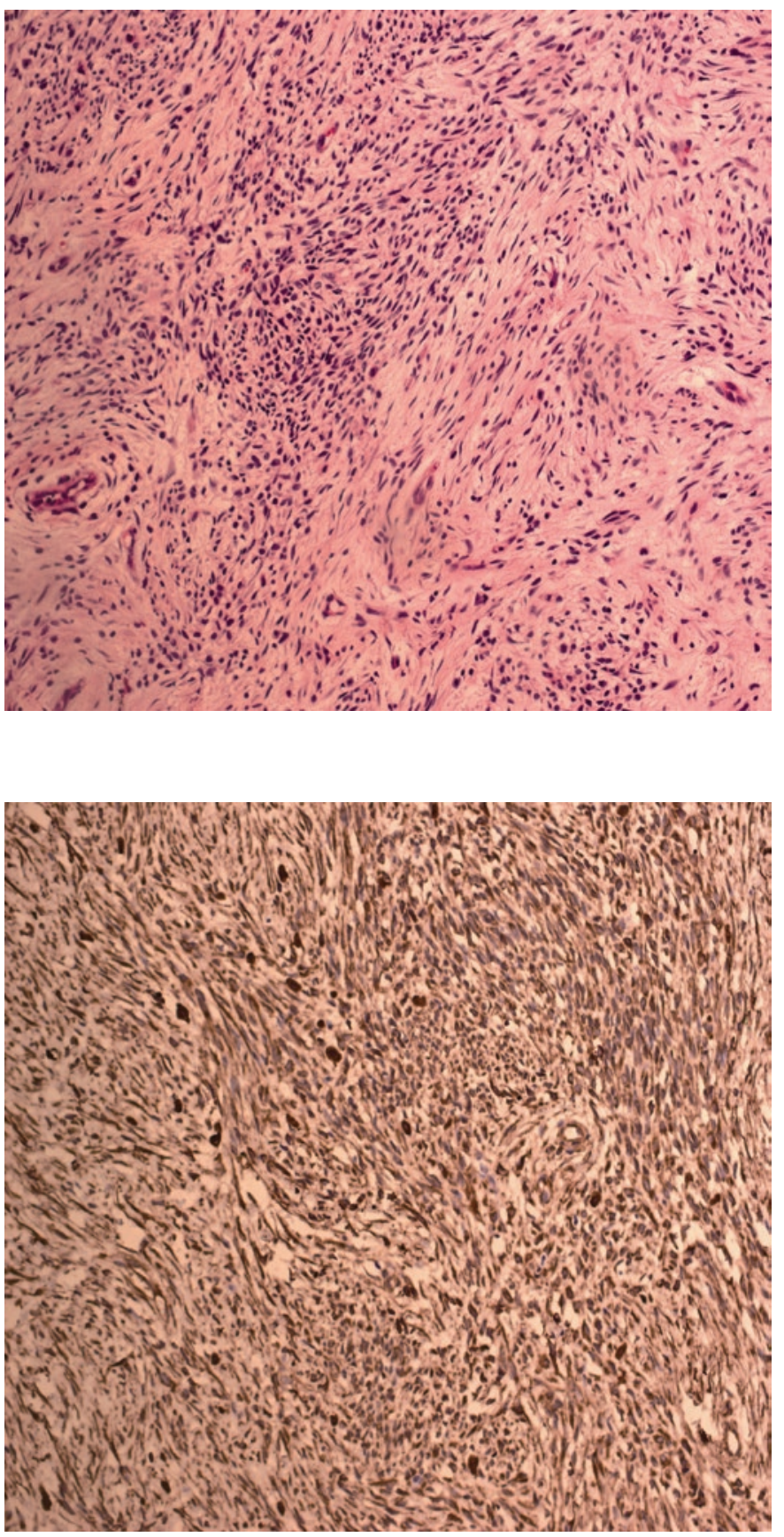

(B)

FIGURE 2. (a) Pathology showed that the tumour consisted of small, uniform spindle cells invading the surrounding muscles (HE×20). (b) Immunohistochemical examination for vimentin was positive (EliVision×20).

ratio was about $4: 1$. The age of the patients ranged from 12 to 79 years at initial presentation, with a mean age of 37 years (data were not available for four patients). ${ }^{2-18}$ Of the 17 patients for whom data were available $2-7,9-18,16(94.1 \%)$ had tumour located in the supraglottic and $1(5.9 \%)$ had tumour in the subglottis; no patient had tumour in the glottis area. For the 16 patients for whom follow-up data were available, the follow-up times ranged from 3 months to 16 years. ${ }^{2-6,8-11,13-18}$ Of these 16 patients, only four (including our patient) developed recurrence and three developed distant metastases. Only two patients (including our patient) died of the disease. The poor outcome might be related to the large extent of the laryngeal synovial sarcoma. ${ }^{18}$

In the majority of cases, the surgical excision with a wide margin is the first treatment choice for synovial carcinoma. The effect of chemotherapy or radiotherapy is controversial. ${ }^{25-27}$ The main treatment regimen for laryngeal synovial sarcoma is surgery, including laryngopharyngectomy, hemilaryngectomy, tumourectomy, total laryngectomy, or tumour resection using a $\mathrm{CO}_{2}$ laser (for localised lesions). ${ }^{3-18}$ Among the patients who underwent $\mathrm{CO}_{2}$ laser surgery, no recurrence or metastasis occurred, and the disease-free survival times were 2 years ${ }^{11}, 3$ years ${ }^{8}$, and 15 months. ${ }^{5}$ The favourable results in this group might be associated with the low volume of the tumours subjected to $\mathrm{CO}_{2}$ laser surgery. $., 8,11$ In cases of laryngeal synovial sarcoma (excluding our case and one case reported by Gatti et al. ${ }^{18}$ ), postoperative chemo/radiotherapy seemed to be effective. ${ }^{2,4,11,14-16}$ No recurrences or metastases occurred, and the longest survival time was 16 years..$^{15}$ Chemo/radiotherapy also seemed to be useful in the treatment of distant metastases and local recurrence. ${ }^{10,14}$ Some reports had a very short follow-up period ( $<2$ years), and the exact outcomes require a further investigation. ${ }^{2,4-6,9,11,13}$ Although our case involved multiple therapeutic strategies, including surgery, radiotherapy, repeated chemotherapies, and targeted therapies, the outcome was poor. This result was similar to that reported by Gatti et al. ${ }^{18}$ Consequently, the ideal treatment has yet to be established because of the limited number of available reports on laryngeal synovial sarcoma.

Magnetic resonance imaging is often recommended as a follow-up modality. ${ }^{21,27}$ Recent studies have indicated that PET is useful in the follow-up of synovial sarcoma. ${ }^{1,23}$ PET/CT has also been used as a diagnostic tool for synovial sarcoma, as well as lymphoma. ${ }^{20,28,29}$ Charest et al. demonstrated $80.0 \%$ sensitivity of PET/CT in 20 soft-tissue synovial sarcomas with a mean $\mathrm{SUV}_{\text {max }}$ of 10.9. ${ }^{28}$ Erturhan et al. reported the use of PET/CT for diagnosis and follow-up in a case of kidney synovial sarcoma. They found slight FDG uptake in the synovial sarcoma and in multiple lymph nodes $\left(\mathrm{SUV}_{\max }=3.5\right)$. PET/ $\mathrm{CT}$ detected no differentiation in multiple lymph nodes in the fourth postoperative month; howev- 


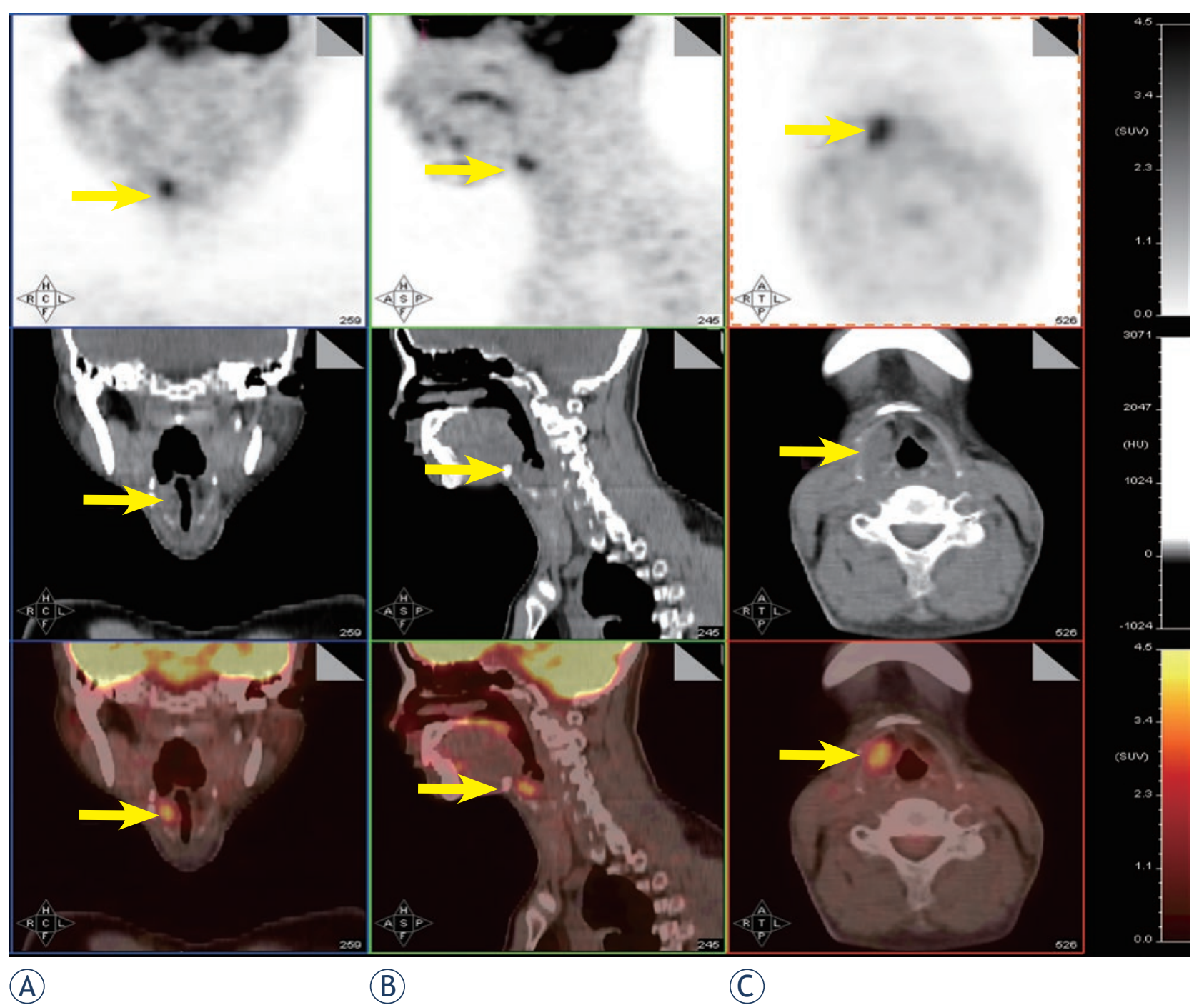

FIGURE 3. PET/CT showed high FDG uptake in the region of the right hypopharynx and laryngeal inlet $\left(S_{U} V_{\max }=4.1\right)$ and no distant metastasis.

er, CT did not show these lymph nodes, and they were reported as normal. ${ }^{23}$ Lisle et al. assessed 44 patients with synovial sarcoma before therapy and resection by FDG-PET. They found that the pretreatment tumour SUV ${ }_{\text {max }}$ predicted the overall and progression-free survival. Patients with $\mathrm{SUV}_{\max }$ $>4.35$ had reduced disease-free survival and were therefore at high risk for local recurrence and metastatic disease. ${ }^{1}$ In our case, although the patient did not undergo pre-treatment PET/CT, three repeated post-treatment PET/CT examinations demonstrated the unsatisfactory treatment outcomes. In this case, $\mathrm{SUV}_{\max }$ did not decrease in response to the various treatments. These findings were consistent with the results of Lisle et al. ${ }^{1}$ Therefore, PET/CT can be a useful workup tool for synovial sarcoma.

\section{Conclusions}

Here, we report a case of laryngeal synovial sarcoma. Although the patient received a comprehensive therapy including surgery, radiotherapy, repeated chemotherapies, and targeted therapies, he had an unfavourable outcome and died of wholebody metastases. Since cases involving the larynx are extremely rare, the treatment of laryngeal synovial sarcoma should follow the guidelines for other tumour sites. To our knowledge, this is the first report of PET/CT findings of laryngeal synovial sarcoma. PET/CT can detect local recurrence and metastasis of laryngeal synovial carcinoma. PET/ $\mathrm{CT}$ can also predict the treatment effect in patients with synovial sarcoma. 

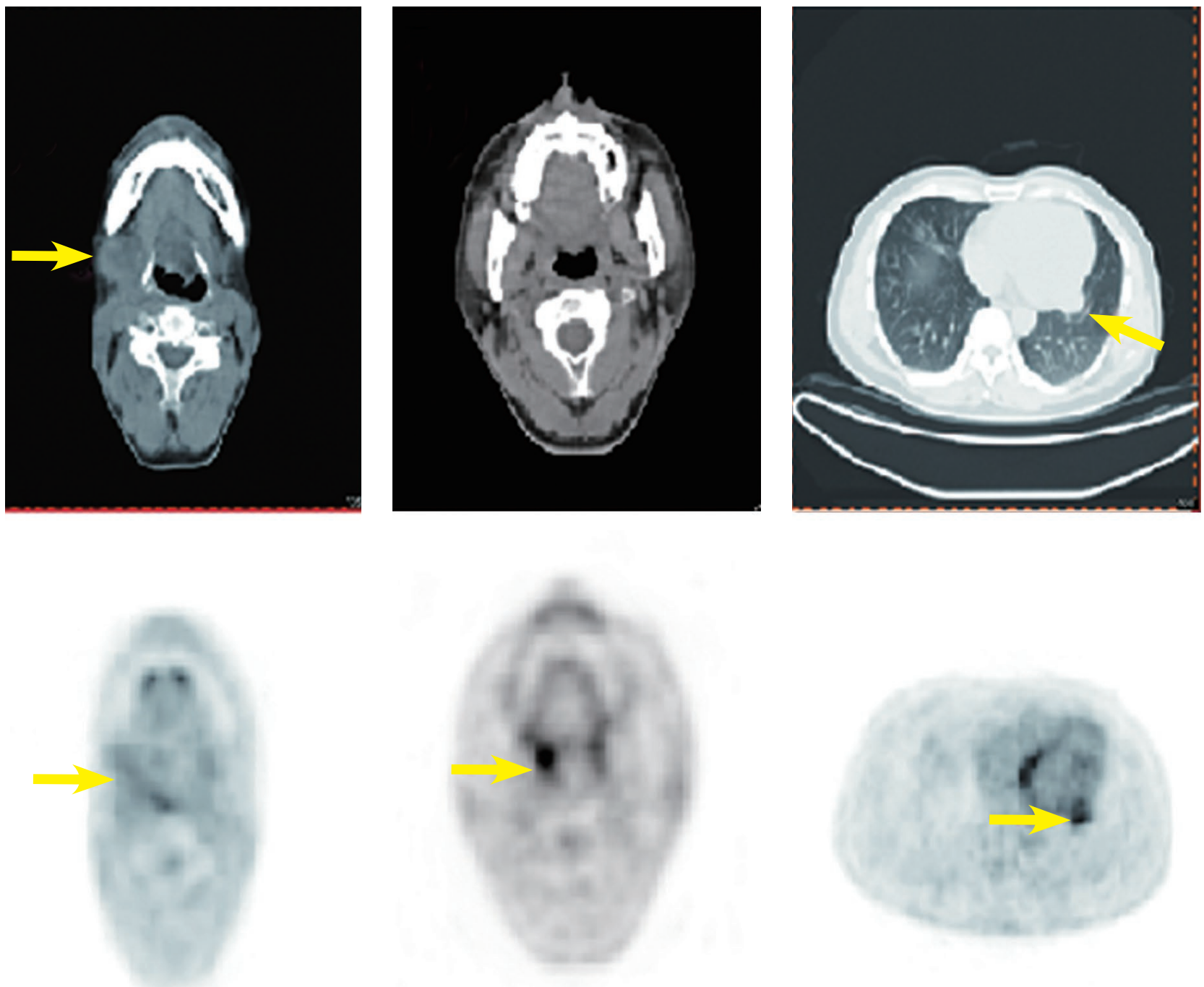

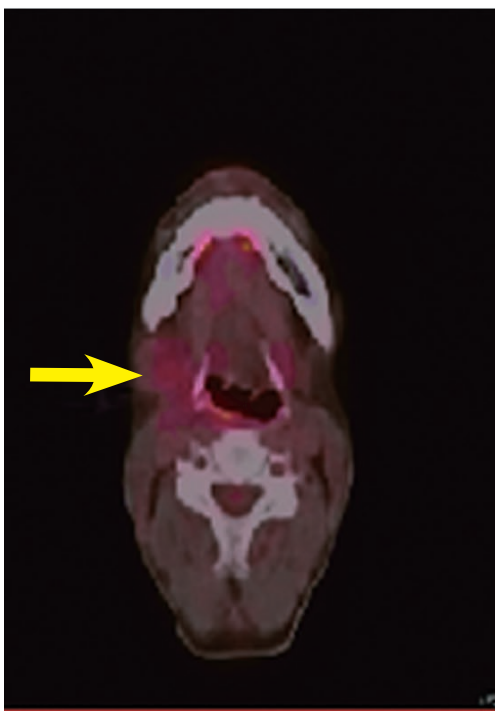

(A)

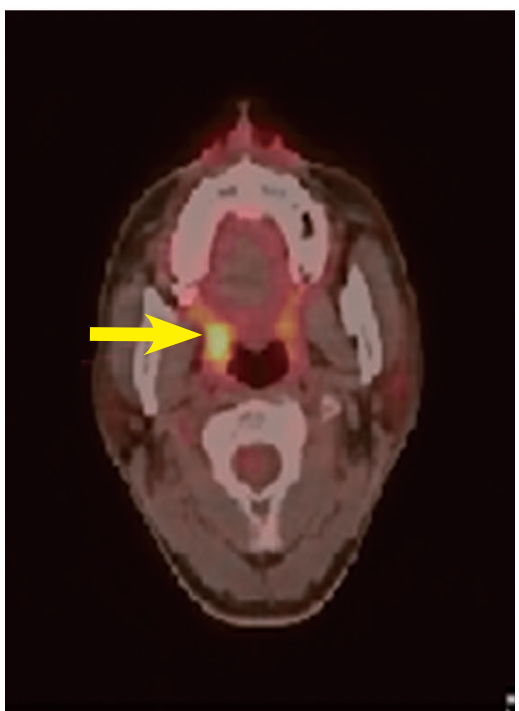

(B)
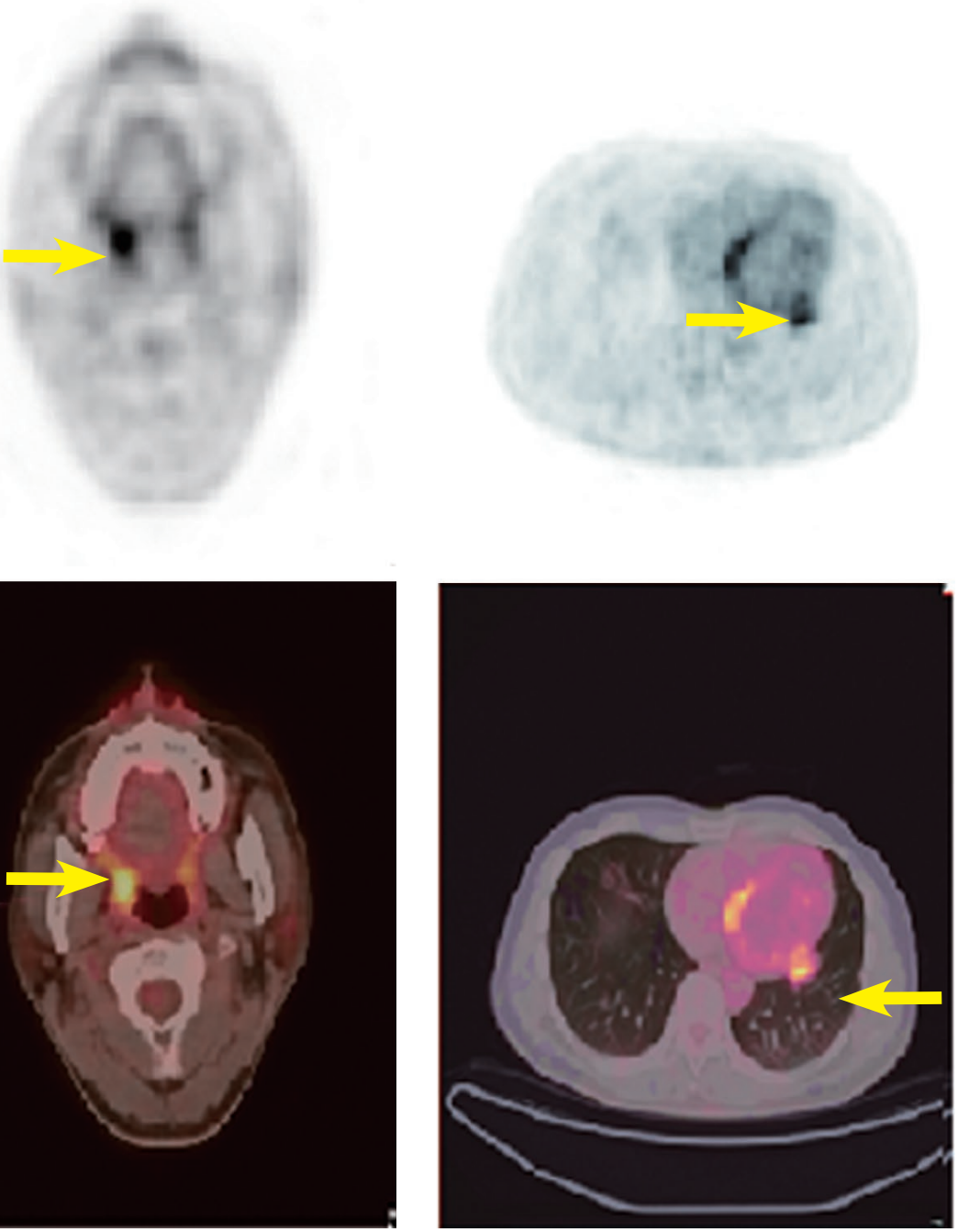

(C)

FIGURE 4. (a, b) Nine months after the second surgery, PET/CT revealed high FDG uptake in the right submaxillary lymph node (SUV $\left.{ }_{\text {max }}=3.2\right)$, right oropharynx $\left(S U V_{\text {max }}=5.4\right)$ and $(c)$ multiple nodules in both lungs $\left(S U V_{\text {max }}=4.6\right)$. 


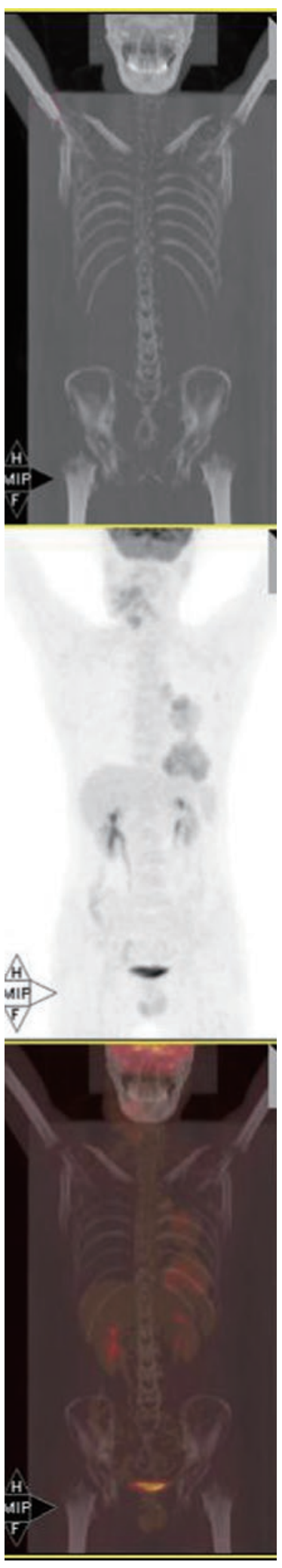

(A)
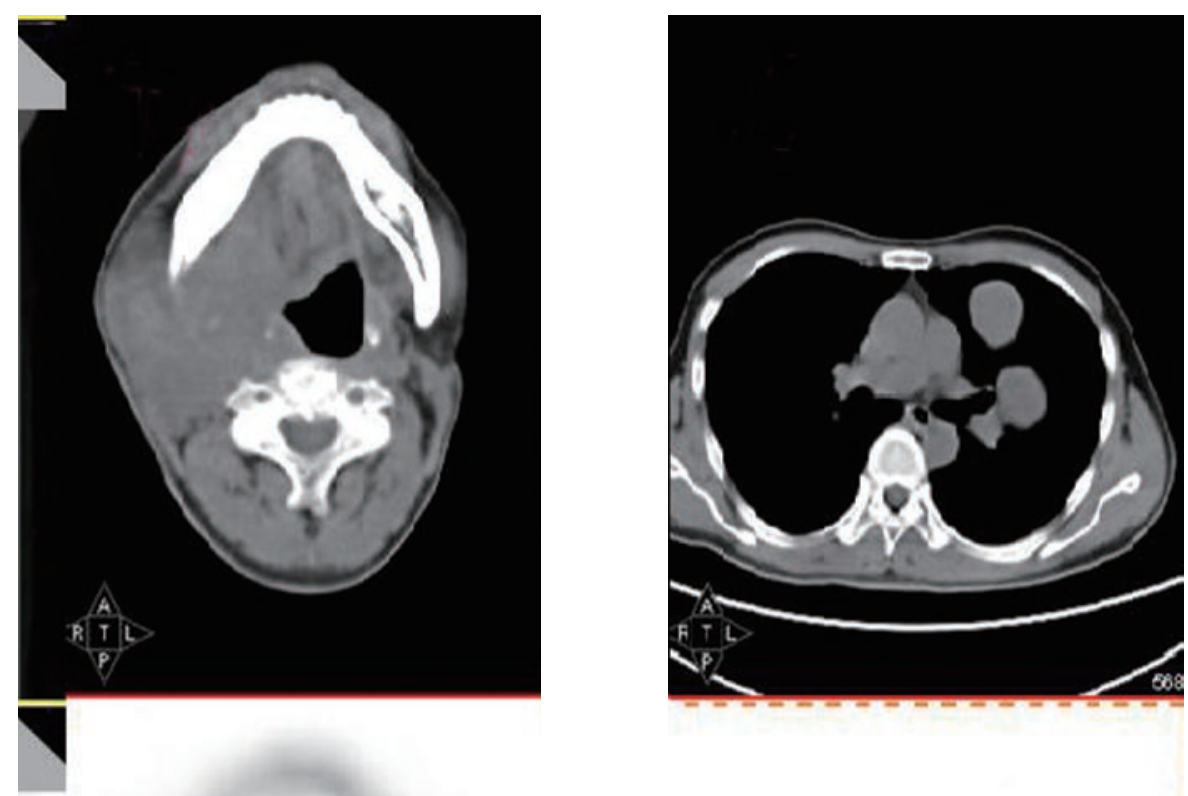

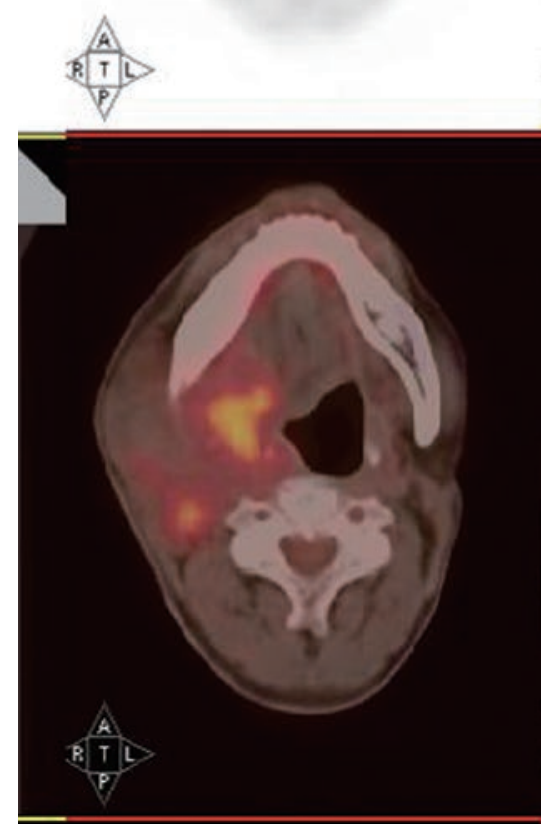

(B)
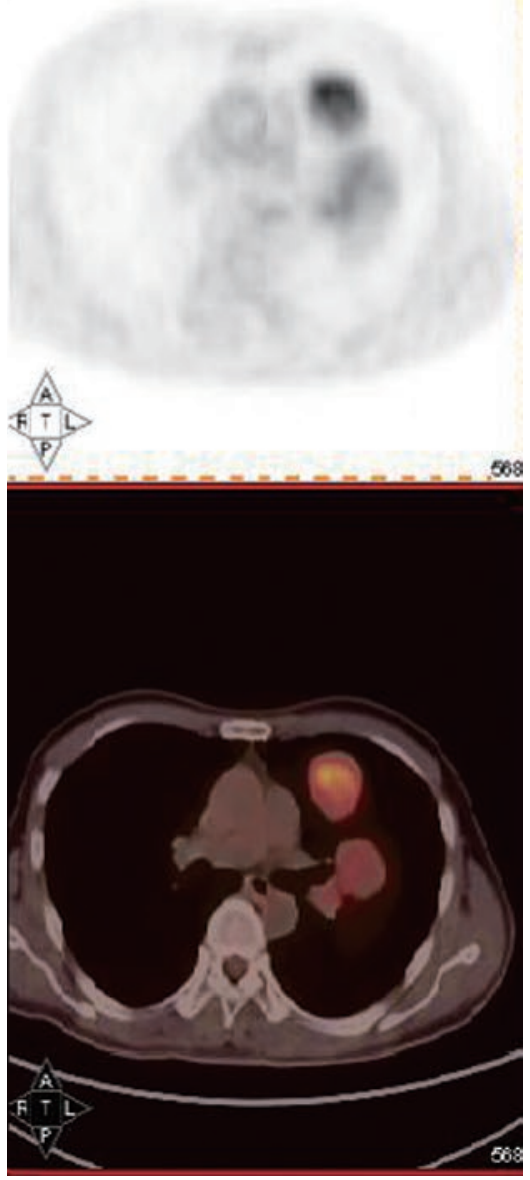

(C)

FIGURE 5. After radiotherapy, repeated chemotherapies and targeted therapies, (a) a whole body scan showed FDG uptake in the right neck and lung. (b) PET/CT revealed a $7.5 \times 9-\mathrm{cm}$ mass in the right submaxillary region with high FDG uptake (SUV ${ }_{\text {max }}=5.2$ ) involving the right parotid gland, right tongue base, right mandible, jugular vein, carotid artery, and surrounding muscles. (c) Multiple nodules in both lungs, were evident with the largest being up to $6.68 \mathrm{~cm}$ in diameter $\left(S U V_{\max }=6.02\right)$. 


\section{References}

1. Lisle JW, Eary JF, O'Sullivan J, Conrad EU. Risk assessment based on FDGPET imaging in patients with synovial sarcoma. Clin Orthop Relat Res 2009; 467: 1605-11.

2. Al-Nemer A, El-Shawarby MA. Laryngeal synovial sarcoma: Case report and literature review. Gulf J Oncolog 2011; 1: 52-6.

3. Miller LH, Santaella-Latimer L, Miller T. Synovial sarcoma of the larynx. Trans Sect Otolaryngol Am Acad Ophthalmol Otolaryngol 1975; 80: 448-451.

4. Fernández-Aceñero MJ, Larach F, Ortega-Fernández C. Non-epithelial lesions of the larynx: review of the 10-year experience in a tertiary Spanish hospital. Acta Otolaryngol 2009; 129: 108-12.

5. Capelli M, Bertino G, Morbini P, Proh M, Falco CE, Benazzo M. CO2 laser in the treatment of laryngeal synovial sarcoma: a clinical case. Tumori 2007; 93: $296-9$

6. Mhawech-Fauceglia P, Ramzy P, Bshara W, Sait S, Rigual N. Synovial sarcoma of the larynx in a 79-year-old woman, confirmed by karyotyping and fluorescence in situ hybridization analysis. Ann Diagn Pathol 2007; 11: 223-7.

7. Abou Zeid HA, Arab SA, Al-Ghamdi AM, Al-Qurain AA, Mokhazy KM. Airway management of a rare huge-size supraglottic mass. Saudi Med J 2006; 27: 711-3.

8. Boniver V, Moreau P, Lefebvre P. Synovial sarcoma of the larynx: case report and literature review. B-ENT 2005; 1: 47-51.

9. Szuhai $\mathrm{K}$, Knijnenburg J, ljszenga $\mathrm{M}$, Tanke $\mathrm{HJ}$, Baatenburg de Jong RJ, Bas Douwes Dekker P, et al. Multicolor fluorescence in situ hybridization analysis of a synovial sarcoma of the larynx with a $\mathrm{t}(\mathrm{X} ; 18)(\mathrm{p} 11.2 ; \mathrm{q} 11.2)$ and trisomies 2 and 8. Cancer Genet Cytogenet 2004; 153: 48-52.

10. Bilgic B, Mete O, Oztürk SA, Demiryont M, Keles N, Basaran M. Synovial sarcoma: a rare tumor of larynx. Pathol Oncol Res 2003; 9: 242-5.

11. Papaspyrou S, Kyriakides G, Tapis M. Endoscopic CO2 laser surgery for large synovial sarcoma of the larynx. Otolaryngol Head Neck Surg 2003; 129: 630-1.

12. Taylor SM, Ha D, Elluru R, El-Mofty S, Haughey B, Wallace M. Synovial sarcoma of the pericricoidal soft tissue. Otolaryngol Head Neck Surg 2002; 126: 428-9.

13. Dei Tos AP, Dal Cin P, Sciot R, Furlanetto A, Da Mosto MC, Giannini C, et al. Synovial sarcoma of the larynx and hypopharynx. Ann Otol Rhinol Laryngol 1998; 107: 1080-5

14. Morland B, Cox G, Randall C, Ramsay A, Radford M. Synovial sarcoma of the larynx in a child: case report and histological appearances. Med Pediatr Oncol 1994; 23: 64-8.

15. Ferlito A, Caruso G. Endolaryngeal synovial sarcoma. An update on diagnosis and treatment. J Otorhinolaryngol Relat Spec 1991; 53: 116-9.

16. Pruszczynski M, Manni JJ, Smedts F. Endolaryngeal synovial sarcoma: case report with immunohistochemical studies. Head Neck 1989; 11: 76-80.

17. Quinn HJ Jr. Synovial sarcoma of the larynx treated by partial laryngectomy Laryngoscope 1984; 94: 1158-61.

18. Gatti WM, Strom CG, Orfei E. Synovial sarcoma of the laryngopharynx. Arch Otolaryngol 1975; 101: 633-6.

19. Ishiki H, Miyajima C, Nakao K, Asakage T, Sugasawa M, Motoi T. Synovia sarcoma of the head and neck: rare case of cervical metastasis. Head Neck 2009; 31: 131-5

20. Polverosi R, Muzzio PC, Panunzio A, Pasquotti G, Schiavon M, Rea F. Synovial sarcoma: CT imaging of a rare primary malignant tumour of the thorax. Radiol Med 2011; 116: 868-75.

21. Rangheard AS, Vanel D, Viala J, Schwaab G, Casiraghi O, Sigal R. Synovial sarcomas of the head and neck: CT and MR imaging findings of eight patients. Am J Neuroradiol 2001; 22: 851-57.

22. Ricard F, Cimarelli S, Deshayes E, Mognetti T, Thiesse P, Giammarile F. Additional Benefit of F-18 FDG PET/CT in the staging and follow-up of pediatric rhabdomyosarcoma. Clin Nucl Med 2011; 36: 672-7.

23. Erturhan S, Seçkiner I, Zincirkeser S, Erbagci A, Celik M, Yagci F, et al. Primary synovial sarcoma of the kidney: use of PET/CT in diagnosis and follow-up. Ann Nucl Med 2008; 22: 225-9.
24. Skytting B. Synovial sarcoma. A Scandinavian sarcoma group profect. Acta Orthop Scand 2000; Suppl. 291: 1-28.

25. Lee N, Shin E. Treatment outcomes for patients with synovial sarcoma of the head and neck. Expert Rev Anticancer Ther 2008; 8: 371-3.

26. Janevska V, Filipovski V, Banev S, Janevski V, Jovcevski A, Spasevska L, et al. Synovial Sarcoma of the liver - a case report. Maced J Med Sc 2011; 4: 185-91.

27. Kusuma S, Skarupa DJ, Ely KA, Cmelak AJ, Burkey BB. Synovial sarcoma of the head and neck: a review of its diagnosis and management and a report of a rare case of orbital involvement. Ear Nose Throat 2010; 89: 280-3.

28. Charest M, Hickeson M, Lisbona R, Novales-Diaz JA, Derbekyan V, Turcotte RE. FDG PET/CT imaging in primary osseous and soft tissue sarcomas: a retrospective review of 212 cases. Eur J Nucl Med Mol Imaging 2009; 36: 1944-51.

29. Huić $D$, Mutvar A, Kinda-Bašić S, Aurer I, Ciglar M, Grošev D, et al. Negative predictive value of F-18-FDG coincidence PET in patients with Hodgkin's disease and a residual mass after therapy: a retrospective diagnostic test study. Radiol Oncol 2009; 43: 258-63. 


\title{
MRI evaluation of tibial tunnel wall cortical bone formation after platelet-rich plasma applied during anterior cruciate ligament reconstruction
}

\author{
Mitja Rupreht ${ }^{1}$, Matjaž Vogrin², Mohsen Hussein ${ }^{3}$ \\ ${ }^{1}$ Radiology Department, University Medical Center Maribor, Medical Faculty, University of Maribor, Slovenia \\ ${ }^{2}$ Orthopaedics Department, University Medical Center Maribor, Medical Faculty, University of Maribor, Slovenia \\ ${ }^{3}$ Artros Center for Orthopaedic Surgery and Sports Medicine, Ljubljana, Slovenia
}

Radiol Oncol 2013; 47(2): 119-124.

Received 29 May 2012

Accepted 6 September 2012

Correspondence to: Mitja Rupreht, MD, PhD, Radiology Department, University Medical Center Maribor, Medical Faculty, University of Maribor, Slovenia. Phone: +386 (0)2 3212 255; Fax: +386 (0)2 3212 982; E-mail: mitja.rupreht@guest.arnes.si

Disclosure: No potential conflicts of interest were disclosed.

Background. After anterior cruciate ligament $(\mathrm{ACL})$ reconstruction, formation of cortical sclerotic bone encircling the femoral and tibial tunnel is a part of intratunnel graft healing. During the physiological cascades of soft tissue healing and bone growth, cellular and hormonal factors play an important role. The purpose of this study was to noninvasively but quantitatively assess the effect of intraoperatively applied platelet-rich plasma (PRP) on the formation of cortical bone encircling the tibial tunnel.

Patients and methods. In fifty patients, standard arthroscopic ACL reconstructions were performed. The PRP group $(n=25)$ received a local application of PRP while the control group $(n=25)$ did not receive PRP. The proximal tibial tunnel was examined by MRI in the paraxial plane where the portion of the tibial tunnel wall circumference consisting of sclerotic cortical bone was assessed with testing occurring at one, two and a half and six months after surgery. Results. At one month after surgery, differences between the groups in the amount of cortical sclerotic bone encircling the tunnel were not significant ( $p=0.928)$. At two and a half months, the sclerotic portion of the tunnel wall in the PRP group (36.2\%) was significantly larger than in the control $(22.5 \%)$ group $(p=0.004)$. At six months, the portion of sclerotic bone in the PRP group (67.1\%) was also significantly larger than in the control (53.5\%) group ( $p=0.003)$. Conclusions. Enhanced cortical bone formation encircling the tibial tunnel at 2.5 and 6 months after ACL graft reconstruction results from locally applied platelet-rich plasma.

Key words: anterior cruciate ligament graft; platelet-rich plasma; tibial tunnel; cortical bone; MRI

\section{Introduction}

After anterior cruciate ligament (ACL) reconstruction, two biological mechanisms take place: ligamentization of the intra-articular part of the graft and healing in the bone tunnel. ${ }^{1-5}$

Among other processes, in the chronic reparative phase of intratunnel graft healing, chondrification, neo-ossification and proliferative osteoblastic activity are present at about 6 weeks after recon- struction $^{3}$ with the formation of new cortical bone, creating the tunnel wall.

During the physiological cascades of soft tissue healing and bone growth, cellular and hormonal factors play an important role, the most important among them being various growth factors (GF).5,6 These proteins have a positive effect on fibroblast proliferation and the synthesis of extracellular matrix proteins and therefore on the enhancement of tissue healing. ${ }^{7-9}$ Platelet-derived growth factors 
(PDGFs) particularly enhance the graft incorporation process. ${ }^{9}$

Platelet-rich plasma (PRP), defined as a portion of the plasma fraction of autologous blood having a platelet concentration above the baseline, contains an autologous concentration of platelets and growth factors. ${ }^{9,10}$ PRP can be activated with thrombin to create platelet-rich plasma gel. The role of local application of various GF in ACL reconstruction has been analysed by previous studies in animals, where their effect has been evaluated with histological findings or biomechanical tests. ${ }^{11-14}$

MRI is because of its excellent contrast resolution $^{15,16}$ feasible method for demonstrating the osteosclerosis, where cortical bone is hypointense on all pulse sequences. To the best of our knowledge, no radiological research has yet evaluated the effect of PRP on the formation of cortical bone encircling the tibial tunnel after ACL reconstruction in humans. We found only one study, which assessed the cortication of the tunnel wall, however, only its presence was evaluated with $\mathrm{CT}$, in comparing two different graft fixation screws. ${ }^{17}$ The enhancing effect of PRP and bone GF on bone formation was demonstrated by histological studies in animal models as well as in periodontology. ${ }^{18-25}$

Therefore, the aim of presented study was to assess the effect of intraoperatively locally applied PRP, using MRI, for quantitative but noninvasive measurement of the cortical sclerotic bone formation, encircling the tibial tunnel. We hypothesized that PRP promotes the tunnel wall cortical bone (TCB) formation that can be quantitatively assessed by MRI.

\section{Patients and methods}

\section{Patient selection, surgical technique and platelet gel preparation}

The study was designed as a 6-month, single-centre trial, approved by the national ethics committee and was carried out in accordance with the ethical standards laid down in the 1964 Declaration of Helsinki. All patients gave their written informed consent for participation in the study.

The 50 patients included in the study, aged between 18 and 50, were treated for ACL rupture. The main indication for reconstruction was a symptomatic, unstable knee joint due to ACL rupture, assessed by the orthopaedic surgeon. All patients with inflammatory diseases, diabetes mellitus, advanced knee osteoarthrosis ( $3^{\text {rd }}$ and $4^{\text {rd }}$ degree), previous knee surgery (osteotomies, reconstructive ligament and meniscal procedures and chondral lesions treatment), malignant diseases, allergy to the contrast media, renal diseases and thrombocytopenia were excluded from the study.

All patients undergoing arthroscopic ACL reconstruction were randomized into 2 groups: PRP group comprised 25 patients ( 15 men, 10 women), and the control group (16 men, 9 women).

All procedures were performed by the same orthopaedic surgeon. In all cases, the standard arthroscopic reconstructive procedure, using the single-incision technique with a double-looped semitendinosus and gracilis tendon graft, was performed. The tunnel sizes in the tibia and femur were matched to the cross-sectional size of the graft and measured 7-9 $\mathrm{mm}$ in diameter. The graft was inserted antegrade via the tibial and femoral tunnel and fixed with 2 bioabsorbable cross pins $(3.2 \mathrm{~mm}$, DePuy Mitek, Massachussets, USA) in the femoral tunnel and with one bioabsorbable interference screw (8-10 mm, DePuy Mitek, Massachusetts, USA) in the tibial tunnel.

The patients in the platelet group received a local application of PRP, which the patients in the control group did not receive. PRP preparation was similar to that previously described. ${ }^{26,27}$ During the surgical procedure; autologous blood was obtained and centrifuged. The fraction of PRP was then mixed with activated autologous human thrombin and applied after autograft positioning, into the femoral and tibial tunnels $(1 \mathrm{ml}$ in each of them), as well as onto the graft itself $(3 \mathrm{ml})$, where the autologous PRP was formed. An interference screw was inserted after PRP application. All patients were blinded to the treatment with PRP. Both groups followed the same standard rehabilitation protocol.

\section{Radiological assessment}

The MRI was performed in proton density (PD) sequence with fat suppression (TR 2900ms, TE $22 \mathrm{ms,}$ 3 NEX, matrix 320×224, FOV 200, slice thickness 3 $\mathrm{mm}, 1 \mathrm{~mm}$ spacing). The tibial tunnel was examined in the paraxial plane, perpendicular to the tunnel axis. The tibial tunnel is more easily identified than the femoral tunnel on scout images, which facilitates planning and analysis. Examinations were performed one, two and a half and six months after the ACL reconstruction.

At the first examination one month after reconstruction, the slice with the most pronounced TCB between the tibial plateau and the tip of the inter- 

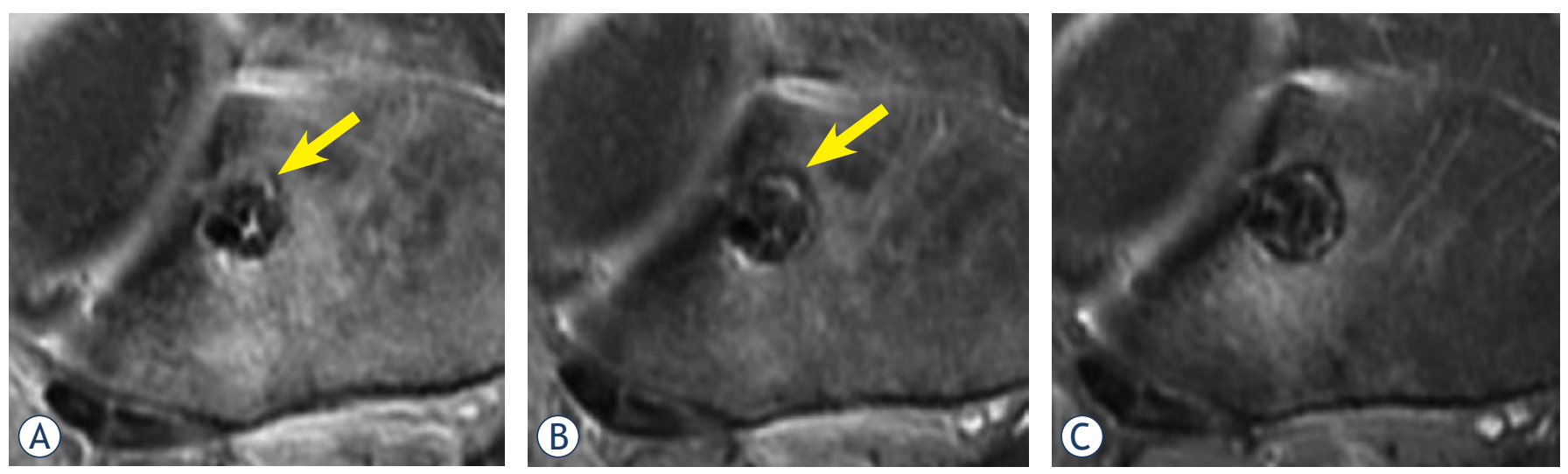

FIGURE 1. Proton-density weighted fat-suppressed paraxial images just below the tibial plateau from the same patient one (A), two and a half (B) and six (C) months after reconstruction. Because of the perpendicular orientation of slices the cross section of the tibial tunnel was in the rule circular. At the first month (A), only small part of the tunnel wall is sclerotic (estimated to be $10 \%$, arrow). At two and a half months (B) about $20 \%$ of the tunnel wall is sclerotic. At six months (C), a thick sclerotic rim encircles estimated $90 \%$ of the tunnel. Note also some high signal intensity surrounding the tunnel, representing oedema, which also decreased during the follow-up.

ference screw, where images were free of volume averaging from the plateau and artefacts from the screw, was chosen for the analysis. In each patient, the same slice was chosen in follow-up assessments.

TCB was defined as a clearly hypointense rim of the tibial tunnel wall that was at least $1 \mathrm{~mm}$ thick, assessed on paraxial slices. The portion of the tunnel wall circumference, consisting of TCB, was assessed by the consensus of the radiologist (M.R.) and the orthopaedic surgeon (M.V.), rounded off to ten percent (Figure 1). The examiners were blinded to group assignment but not to the time of the procedure.

\section{Statistical analysis}

Numerical data are presented as mean values, while categorical data are expressed as proportions. Differences in TCB between the platelet and control groups were analysed using the MannWhitney test. Changes in TCB from the first to the final examination after ACL reconstruction were analysed by the Friedman two-way analysis of variance. As the post-hoc tests, Wilcoxon's signed ranks tests with Keppels modification of the Bonferroni correction of alpha were used. A P value less than 0.05 were taken to represent statistical significance. Data were analysed using PASW 18 software (SPSS Inc., Chicago, IL, USA).

\section{Results}

20 patients from the control group and 21 patients from the PRP group were available for the followup and were analysed, while 9 patients from initial group were lost to follow-up. A comparison of the preoperative parameters of remaining patients in the PRP and the control groups showed that both groups were comparable in gender, age, injury site and body mass index (Table 1).

We observed a gradual increase in the percentage of the tunnel wall consisting of TCB during the follow-up (Table 2). In each group, post-hoc comparisons showed a significant increase in average

TABLE 1. Preoperative data on injured patients

\begin{tabular}{|c|c|c|c|}
\hline Characteristic & $\begin{array}{l}\text { Control group } \\
(n=20)\end{array}$ & $\begin{array}{l}\text { PRP group } \\
\quad(n=21)\end{array}$ & $\begin{array}{l}\text { p-value for differences } \\
\text { between groups }\end{array}$ \\
\hline Men, n (\%) & $15(75)$ & $13(62)$ & 0.505 \\
\hline Age, years & $32.6 \pm 12.3$ & $37.2 \pm 8.4$ & 0.112 \\
\hline Injured knee, n (\%) & & & 1.000 \\
\hline Right & $12(60 \%)$ & $12(57 \%)$ & \\
\hline Left & $8(40 \%)$ & 9 (43\%) & \\
\hline BMI & $24.5 \pm 2.1$ & $26.5 \pm 4.1$ & 0.078 \\
\hline
\end{tabular}

Numerical data are expressed as mean \pm standard deviation; $\mathrm{BMI}=$ body mass index 
TABLE 2. Percentage of tibial tunnel wall cortical bone in both groups at follow-up examinations. Values are given as means $(95 \%$ confidence interval)

\begin{tabular}{cccc}
\hline Period from surgery (months) & $\begin{array}{c}\text { Control group } \\
(\mathrm{n}=\mathbf{2 0})\end{array}$ & $\begin{array}{c}\text { PRP group } \\
(\mathbf{n}=21)\end{array}$ & $\begin{array}{c}\text { p-values for differences } \\
\text { between groups }\end{array}$ \\
\hline 1 & $5.0(1.8-8.2)$ & $6.7(1.8-11.5)$ & 0.928 \\
2.5 & $22.5(17.3-27.7)$ & $36.2(28.7-43.7)$ & 0.004 \\
6 & $53.5(47.0-60.0)$ & $67.1(61.0-73.3)$ & 0.003 \\
\hline
\end{tabular}

values of TCB between the three control examinations with increasing postoperative time $(\mathrm{p}<0.001$ in all paired comparisons).

At the first postoperative month we found only small amount of TCB in each group with a nonsignificant difference between the groups $(\mathrm{p}=0.928)$. At two and a half months as well as at six months after surgery, the mean percentage of TCB (Table 2) was significantly higher in the PRP group than in the control group (36.2 vs 22.5 and 67.1 vs 53.5, p = 0.004 and $\mathrm{p}=0.003$, respectively).

\section{Discussion}

For ethical reasons, histological evaluation of ACL graft incorporation in humans is impossible, particularly in the bone tunnels. However, MRI is a method of choice for the evaluation of the knee. ${ }^{28}$ Graft healing in the tibial tunnel starts immediately after the operation as an acute inflammatory response with oedema, neutrophils and recruited macrophages present in the tendon bone interface as early as 4 days after surgery. ${ }^{2,3}$ At 3 weeks, small vessels appear along with an increased number of osteoblasts on the bone surface. After 6 weeks, the vessels decrease in number and there is a shieldlike new bone formation surrounding the graft as well as an increased number of collagen fibers integrating along the tendon. ${ }^{6}$ Sharpey-like fibers, which anchor the fibroproliferative process to the bone, were found at 6 to 12 weeks after reconstruction in a dog model, followed by progressive bone ingrowth. ${ }^{2,3}$ The tissue maturation process is finished at about 26 weeks, although this differs among various animals and also between different types of grafts. ${ }^{1,2,4}$

In the intratunnel graft healing process, incorporation progresses through the formation of a new matrix at the tendon-bone interface. Proliferation of new bone trabeculae along the edge of the tunnel is seen as early as three weeks after surgery. ${ }^{6}$ This pattern is not uniform, as some areas exhibit a cartilaginous interface between tendon and bone. The zone of fibrocartilage may persist and represents a form of direct healing by tissue which may undergo enchondral bone formation. ${ }^{6}$ This histological evidence is consistent with our observation of focal areas of sclerosis, representing the TCB, which in the follow-up period in each group expanded and finally fused. Although, on average, two thirds of the tibial wall circumference were sclerotic in the PRPG group at six months, we did not detect complete TCB formation surrounding the entire tunnel in any patient. This could be the consequence of a relatively short follow-up period and is in accordance with the observation of increased osteoblastic activity up to two years after reconstruction. ${ }^{4}$

Several reports of the enhancing effect of PDGF and various other GFs on bone proliferation exist, particularly in periodontology. ${ }^{11-25}$ These factors have been shown to enhance murine osteoblast activity and proliferation in vitro. ${ }^{23}$ In vivo, 2-fold increases in uptake of the bone-seeking radiopharmaceutical Technetium 99-MDP, as well as histologically up to a 10-fold increase in new bone and cement were found in dogs. ${ }^{21}$ Locally added PRP accelerated bone healing after mandibular reconstruction in goats considerably. ${ }^{25}$ In humans, significant increase in alveolar bone formation in the process of periodontal regeneration was demonstrated as effects of locally applied PDGF and insulin-like GF. ${ }^{22,24}$

Despite the lack of radiological evidence in humans, assessment with MRI in sagittal and coronal slices demonstrated significantly more periosteal and intratunnel bone formation as effects of locally applied combination of bone GF during the ACL reconstruction in rabbits. This was histologically confirmed with more extensive new bone trabeculae and cartilage formation at the tendon-bone interface at two and eight weeks after reconstruction and generally more mature tissue at the grafttunnel interface. ${ }^{18}$ In addition, other histological studies in animal models also showed more new bone formation at this interface as effects of local application of bone morphogenetic protein (BMP)7 and BMP-2 up to eight weeks after reconstruc- 
tion. ${ }^{19,20}$ Moreover, all of the above-mentioned studies also found higher tensile strength in bone GF groups.

We did not detect differences in the TCB between the groups at one month, which is not in agreement with the above-mentioned animal studies, possibly because of a higher sensitivity with histological analysis, which may detect small changes in the TCB in the first weeks after surgery, when changes are too subtle for observation with MRI. The assessment of joint or even graft stability was not a part of the study protocol which could represent a limitation. However, increased anterior knee stability resulting from PDGF or bone GF has been demonstrated in various studies of humans and animals, ${ }^{18-20,27}$ although the clinical effect of the PRP is still debated. ${ }^{26,29}$ There are several other limitations of the study. We did not evaluate the femoral tunnel because such examination would have to be performed in a different plane, and would therefore significantly prolong the examination. In the tibial tunnel, the analysis of additional neighbouring slices could be more accurate, but also time-consuming, therefore in each patient the same slice was meticulously selected for analysis in all follow-up examinations. Owing to superior spatial resolution with even thinner slices, CT examination could be possibly more accurate, but at the cost of significant radiation exposure, important particularly in this young study population. We did not perform preoperative MRI, nor did we evaluate for lower degrees of osteoarthrosis and concurrent procedures during arthroscopy, as well as injury chronicity and possible drugs intake like NSAR and corticosteroids. All these factors could influence the inflammatory response in the healing process, which is, however, rather questionable in the intratunnel region compared with intra-articular inflammation.

In conclusion, the results of our study demonstrate that formation of focal areas of sclerotic cortical bone with subsequent fusion into a thick tibial tunnel wall is a part of the ACL graft incorporation process that can be quantitatively assessed by MRI. Furthermore, we observed that local application of PRP results in enhanced cortical bone formation encircling the tibial tunnel at 2.5 and 6 months but not 1 month after ACL reconstruction.

\section{References}

1. Arnoczky SP, Tarvin GB, Marshall JL. Anterior cruciate ligament replacement using patellar tendon. An evaluation of graft revascularisation in dog. J Bone Joint Surg Am 1982; 64: 217-24.
2. Rodeo SA, Arnoczky SP, Torzilli PA, Hidaka C, Warren RF. Tendon-healing in a bone tunnel. A biomechanical and histological study in a dog. J Bone Joint Surg Am 1993; 75: 1795-803.

3. Scranton PE Jr, Lanzer WL, Ferguson MS, Kirkman TR, Pflaster DS. Mechanisms of anterior cruciate ligament neovascularisation and ligamentisation. Arthroscopy 1998; 14: 702-16.

4. Goradia VK, Rochat MC, Grana WA, RohrerMD, Prasad HS. Tendon-to-bone healing of a semitendinosus tendon autograft used for $\mathrm{ACL}$ reconstruction in a sheep model. Am J Knee Surg 2000; 13: 143-51.

5. Kawamura S, Ying L, Kim HJ, Dynbyl C, Rodeo SA. Macrophages accumulate in the early phase of tendon-bone healing. J Orthop Res 2005; 23: 1425-32.

6. Deehan DJ, Clawston TE. The biology of integration of the anterior cruciate ligament. J Bone Joint Surg Br 2005; 87: 889-95.

7. Heldin $\mathrm{CH}$, Westermark B. PDGF-like growth factors in autocrine stimulation of growth. J Cell Physiol Suppl 1987; Suppl 5: 31-4.

8. Anitua E, Andia I, Ardanza B, Nurden P, Nurden AT. Autologous platelets as a source of proteins for healing and tissue regeneration. Thromb Haemost 2004; 91: 4-15.

9. Mehta S, Watson JT. Platelet rich concentrate: basic science and current clinical applications. J Orthop Trauma 2008; 22: 432-8.

10. Marx RE. Platelet-rich plasma (PRP): what is PRP and what is not PRP? Implant dent 2001; 10: 225-8.

11. Weiler A, Forster C, Hunt P, Falk R, Jung T, Unterhauser FN, et al. The influence of locally applied platelet-derived growth factor-BB on free tendon graft remodeling after anterior cruciate ligament reconstruction. $\mathrm{Am} J$ Sports Med 2004; 32: 881-91.

12. Yamazaki S, Yasuda K, Tomita F, Tohyama H, Minami A. The effect of transforming growth factor-beta1 on intraosseous healing of flexor tendon autograft replacement of anterior cruciate ligament in dogs. Arthroscopy 2005; 21: 1034-41.

13. Yoshikawa $T$, Tohyama $H$, Katsura $T$, Kondo $E$, Kotani $Y$, Matsumoto $H$, et al. Effects of local administration of vascular endothelial growth factor on mechanical characteristics of the semitendinosus tendon graft after anterior cruciate ligament reconstruction in sheep. Am J Sports Med 2006; 34: 1918-25.

14. Nagumo A, Yasuda K, Numazaki H, Azuma H, Tanabe Y, Kikuchi S, et al Effects of separate application of three growth factors (TGF-beta1, EGF and PDGF-BB) on biomechanical properties of the in situ frozen-thawed anterior cruciate ligament. Clin Biomech (Bristol, Avon) 2005; 20: 283-90.

15. Xu J, Shen J, Ding Y, Shen HY, Zeng ZP, Ma RF, et al. The clinical value of combined use of MR imaging and multi-slice spiral $\mathrm{CT}$ in limb salvage surgery for orthopaedic oncology patients: initial experience in nine patients. Radiol Oncol 2012; 46: 189-97.

16. Wang $\mathrm{X}, \mathrm{Xu} \mathrm{M}$, Liang $\mathrm{H}, \mathrm{Xu}$ L. Comparison of $\mathrm{CT}$ and MRI in diagnosis of cerebrospinal leak induced by multiple fractures of skull base. Radiol Oncol 2011; 45: 91-6.

17. Robinson J, Huber C, Jaraj P, Colombet P, Allard M, Meyer P. Reduced bone tunnel enlargement post hamstring $\mathrm{ACL}$ reconstruction with poly-L-lactic acid/hydroxyapatite bioabsorbable screws. Knee 2006; 13: 127-31.

18. Anderson K, Seneviratne AM, Izawa K, Atkonson BL, Potter HG, Rodeo SA. Augmentation od tendon healing in an intraarticular bone tunnel with use of a bone growth factor. Am J Sports Med 2001; 29: 689-98.

19. Mihelič R, Pecina $M$, Jelic $M$, Zoricic $S$, Kusec V, Simic $P$, et al. Bone morphogenetic protein-7 (osteogenic protein-1) promotes tendon graft integration in anterior cruciate ligament reconstruction in sheep. Am J Sports Med 2004; 32: 1619-25.

20. Rodeo SA, Suzuki K, Deng XH, Wozney J, Warren RF. Use of recombinant human bone morphogenetic protein-2 to enhance tendon healing in a bone tunnel. Am J Sports Med 1999; 27: 476-88.

21. Lynch SE, de Castilla GR, Williams RC, Kiritsy CP, Howell TH, Reddy MS, et al The effects of short-term application of a combination of platelet-derived and insulin-like growth factors on periodontal wound healing. I Periodontol 1991; 62: 458-67.

22. Howell TH, Fiorellini JP, Paquette DW, Offenbacher S, Giannobile WV, Lynch SE. A phase I/II clinical trial to evaluate a combination of recombinant human platelet-derived growth factor-BB and recombinant human insulin-like growth factor-I in patients with periodontal disease. J Periodontol 1997; 68: 1186-93. 
23. Mott DA, Mailhot J, Cuenin MF, Sharawy M, Borke J. Enhancement of osteoblast proliferation in vitro by selective enrichment of demineralized freeze-dried bone allograft with specific growth factors. J Oral Implantol 2002; 28: 57-66.

24. Nevins M, Camelo M, Nevins ML, Schenk RK, Lynch SE. Periodontal regeneration in humans using recombinant human platelet-derived growth factor-BB (rhPDGF-BB) and allogenic bone. J Periodontol 2003; 74: 1282-92.

25. Fennis JP, Stoelinga PJ, Jansen JA. Mandibular reconstruction: a histological and histomorphometric study of the use of autogenous scaffolds, particulate cortico-cancelous bone grafts and platelet rich plasma in goats. Int Oral Maxillofac Surg 2004; 33: 48-55.

26. Nin JR, Gasque GM, Azcárate AV, Beola JD, Gonzales MH. Has platelet-rich plasma any role in anterior cruciate ligament allograft healing? Arthroscopy 2009; 25: 1206-13.

27. Vogrin $M$, Rupreht $M$, Dinevski D, Hašpl $M$, Kuhta $M$, Jevsek $M$, et al. Effects ofa platelet gel on early graft revascularization after anterior cruciate ligament reconstruction: a prospective, randomized, double-blind, clinical trial. Eur Surg Res 2010; 45: 77-85.

28. Vasilevska V, Szeimies U, Staebler A. MRI diagnostis of Baker cyst and significance of associated medial compartment knee osteoarthritis. Radiol Oncol 2008; 42: 51-8.

29. Vavken P, Sadoghi P, Murray MM. The effect of platelet concentrates on graft maturation and graft-bone interface healing in anterior cruciate ligament reconstruction in human patients: a systematic review of controlled trials. Arthroscopy 2011; 27: 1573-83. 


\title{
An incidental case of biliary fascioliasis with subtle clinical findings: US and MRCP findings
}

\author{
Hakan Önder, Faysal Ekici, Emin Adin, Suzan Kuday, Hatice Gümüș, Aslan Bilici \\ Department of Radiology, Dicle University Medical Faculty, Diyarbakır, Turkey
}

Radiol Oncol 2013; 47(2): 125-127.

Received 6 March 2012

Accepted 20 September 2012

Correspondence to: Hakan Önder, MD, Dicle Üniversitesi Tıp Fakültesi, Radyoloji Anabilim Dalı, 21280 Diyarbakır, Turkey. Phone: +395333242691; Fax: +904122488523; E-mail: drhakanonder@hotmail.com

Disclosure: No potential conflicts of interest were disclosed.

Background. Fascioliasis is a disease caused by the trematode Fasciola hepatica. Cholangitis is a common clinical manifestation. Although fascioliasis may show various radiological and clinical features, cases without biliary dilatation are rare.

Case report. We present unique ultrasound (US) and magnetic resonance cholangiopancreatography (MRCP) findings of a biliary fascioliasis case which doesn't have biliary obstruction or cholestasis. Radiologically, curvilinear parasites compatible with juvenile and mature Fasciola hepatica within the gallbladder and common bile duct were found. The parasites appear as bright echogenic structures with no acoustic shadow on US and hypo-intense curvilinear lesions on T2 weighted MRCP images.

Conclusions. Imaging studies may significantly contribute to the diagnosis of patients with subtle clinical and laboratory findings, particularly in endemic regions.

Key words: fascioliasis; ultrasonography; magnetic resonance cholangiopancreatography

\section{Introduction}

Hepatobiliary fascioliasis is caused by the trematode Fasciola hepatica (F. hepatica). The worldwide increase in the diagnosis of fascioliasis is related to the increased availability and use of ultrasonography, awareness of the important role of imaging in diagnosis, and the recent development of specific serologic tests. The parasite is common in sheep, goat and cattle. It is transmitted to humans via contaminated water or green vegetables such as watercress. The disease is endemic in some Middle and Far East countries and in some parts of Central and South America. ${ }^{1,2}$

Human fascioliasis mainly involves the hepatobiliary system. It has two different phases: hepatic (acute), and biliary (chronic) phase. The hepatic phase of the disease occurs when immature parasites pass into the liver through its capsule. The parasites migrate through the liver parenchyma to the biliary system. Biliary phase of the disease occurs in the presence of parasites in the biliary system. ${ }^{2,3}$
Typical radiological findings of biliary fascioliasis have been reported previously. ${ }^{4,5}$ Herein we present a case of biliary fascioliasis with no biliary obstruction and cholestasis; unique MRCP and US imaging findings.

\section{Case report}

33-year-old female patient from the south-east part of Turkey has been referred to the department of gastroenterology with a stomach ache complaint. Laboratory results and physical examination were normal, except tenderness in the right upper quadrant and high erythrocyte sedimentation ratio.

MRCP revealed heterogeneous intensities within the gallbladder along with mild dilatation of common bile duct and intra-ductal linear hypointense lesion which was reached at $2 \mathrm{~cm}$ length on T2 weighted series (Figure 1A, B). Heterogeneous lesions at right liver lobe with maximum size of 1.5 $x 1 \mathrm{~cm}$ were also noted. F. hepatica was suspected 


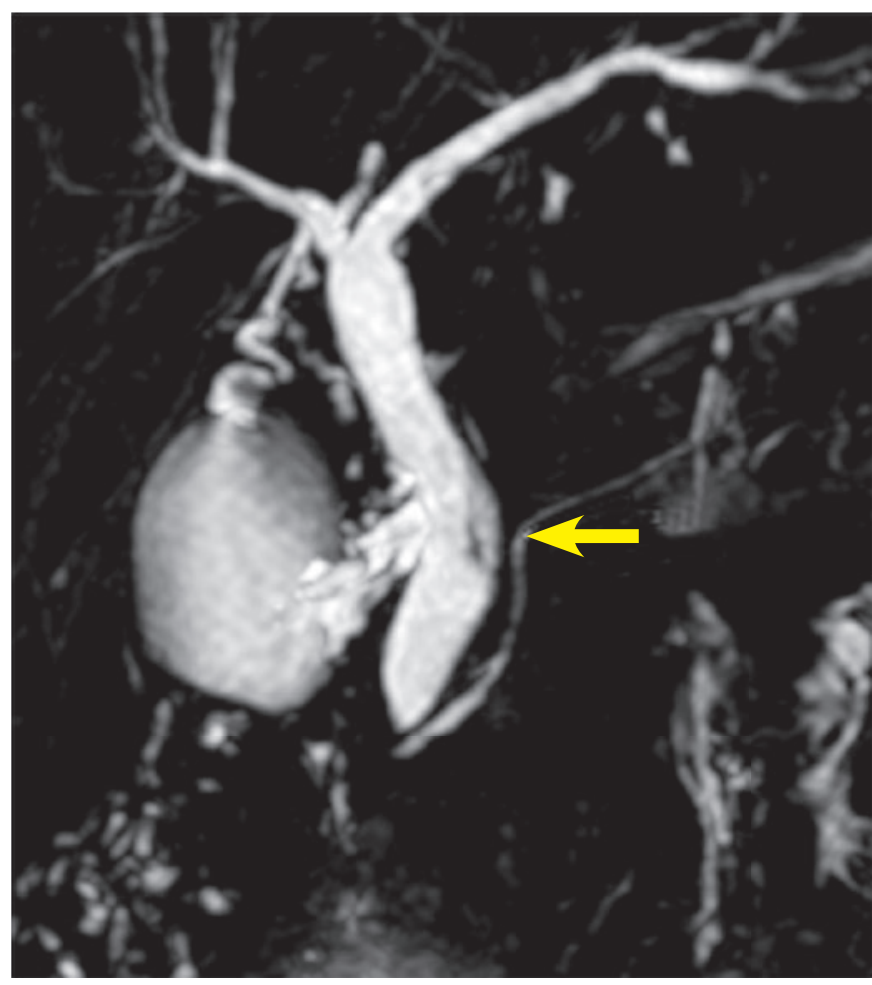

FIGURE 1A. MRCP image shows mild dilation of the common bile duct and linear hypointense signal changes in the distal common bile duct (arrow).

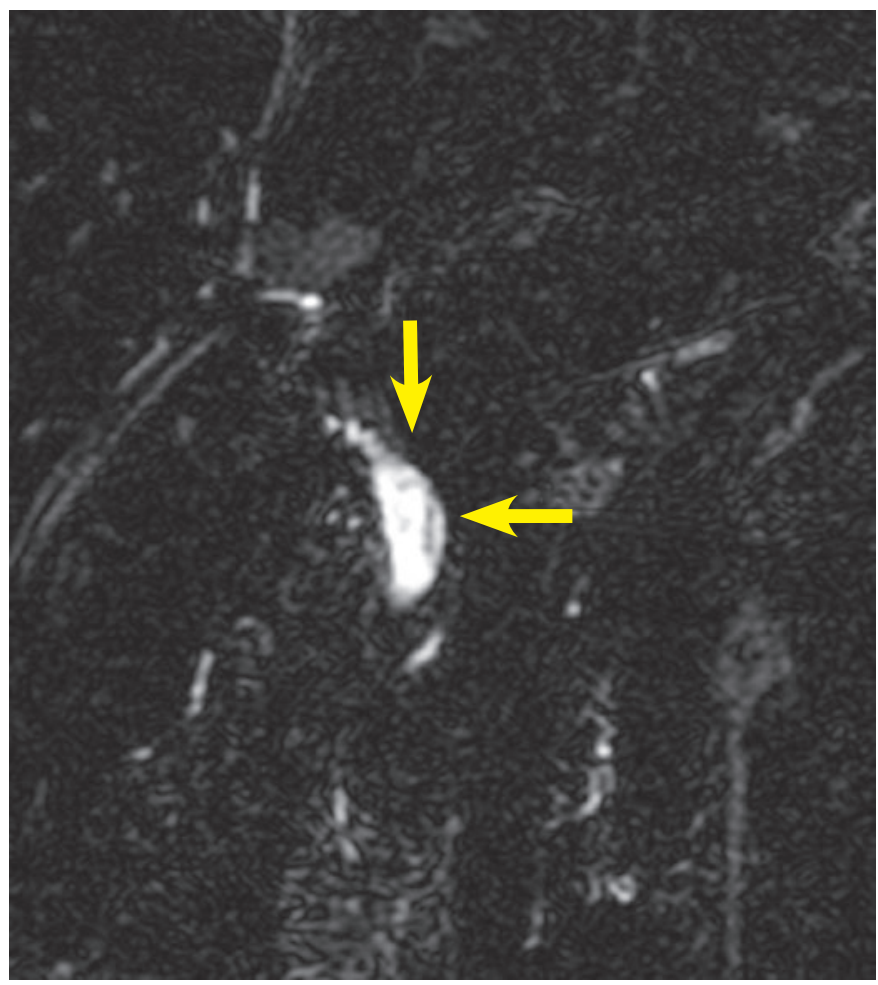

FIGURE 1B. Thick-section (7 mm) MRCP image demonstrates the curvilinear parasite. since our region was endemic for that parasite. Three days after MRCP imaging, the US examination of the patient had depicted multiple mature $F$. hepatica parasites in the gallbladder and main bile ducts lumen with approximately $2 \mathrm{~cm}$ mean size (Figure 2A,B). Stool sample was (+) for F. hepatica and Helicobacter pylori. In ELISA testing, the result was read photometrically at $450 \mathrm{~nm}$ (Tecan Sunrisemicro ELISA). The absorbance value of the patient was 18 DRG Units=DU/ml. The excretory/ secretory antigens was used for immuno-diagnosis of fascioliasis in the kit (values greater than 11.0 DRG Units=DU/ml are interpreted as seropositive, cut-off value 10).

The patient received $10 \mathrm{mg} / \mathrm{kg}$ of triclabendazole therapy per day. Six weeks after initial treatment, erythrocyte sedimentation rate value returned to normal and parasites were not seen any more with the US and MRCP investigation.

\section{Discussion}

F. hepatica is a zoonosis which can rarely infect people who have an oral contact with water or water plants contaminated by the larvae. Two different phases of fascioliasis with distinct signs and symptoms have been described. Hepatic (acute) phase is characterized by right upper quadrant pain, hepatomegaly, intermittent fever, urticaria and marked eosinophilia. In biliary (chronic) phase, patients have dyspeptic symptoms and intermittent right upper quadrant pain with or without cholestasis. Overlaps between both phases may occur. ${ }^{6-8}$

The diagnosis of fascioliasis is based on clinical symptoms, stool examination, serological and radiological studies the egg production rate of $F$. hepatica is low and. Therefore, stool examination is not very sensitive. Presently, serological studies are the main diagnostic tool and the most common serological method is ELISA which detects antibodies to the excretory-secretory antigen products from $F$. hepatica. ${ }^{9,10}$ Our patient had both positive serological tests and stool examination.

The presence of parasites in the biliary system in the chronic phase of the disease, is characterized by cholangitic fevers and dyspepsia caused by partial biliary obstruction. In this phase, US is able to demonstrate the floating parasites in the biliary system and accompanying oedema of gallbladder or distal bile duct. Parasites usually appear as leaf-like or snail-like non-shadowing oval echogenic structures. ${ }^{6-8}$ In our case, intraluminal 
floating parasites were visible but concomitant biliary system oedema was not present.

In case series of five patients, Koç et al. found hypo-intense expansive filling defect due to F. hepatica on T2W images on MRCP of one particular patient. ${ }^{11}$ Linear hypo-intense appearance with no luminal expansion on MRCP was seen in images of our patient.

Parasites have variable length between 5 and 25 $\mathrm{mm}$, but their characteristic size is $10 \mathrm{~mm}$. In our case parasites were not at the same location on US and MRCP images. In our opinion it might be due to the movement of parasites during the time period between the examination dates.

Cholangitis is a common clinical manifestation of biliary fascioliasis. Gallbladder or common bile duct wall thickening along with mild intrahepatic biliary ductal dilatation frequently occur in these cases. $8,9,12$ Mild dilatation of common bile duct with no apparent oedema was found in our case.

In conclusion, fascioliasis should be a differential diagnosis when bile duct dilatation or intraluminal curvilinear structures are encountered on the US or MRCP images of patients with subtle clinical findings, particularly at endemic regions. Imaging findings should be supported by ELISA testing. Fascioliasis may show various radiological features and radiologists should be aware of this information.

\section{References}

1. Arjona R, Riancho JA, Aguado JM, Salesa R, Gonzales-Macias J. Fascioliasis in developed countries: a review of classic and aberrant forms of the disease. Medicine 1995; 74: 13-23.

2. Aksoy DY, Kerimoglu U, Oto A, Ergüven $S$, Arslan $S$, Unal S, et al. Infection with Fasciola hepatica. Clin Microbiol Infect 2005; 11: 859-61.

3. Pagola Serrano MA, Vega A, Ortega E, Gonzalez A. Computed tomography of hepatic fascioliasis. J Comput Assist Tomogr 1987; 11: 269-72.

4. Yeşildağ A, Şenol A, Köroğlu M, Koçkar C, Oyar O, Işler M. Hepatobiliary fascioliasis: a case with unusual radiological features. Diagn Interv Radiol 2010; 16: 299-301.

5. Yesildağ A, Yıldız H, Demirci $M$, Gören I, Isler M. Biliary Fascioliasis: Sonographic appearance patterns. J Clin Ultrasound 2009; 37: 26-30.

6. Han JK, Choi BI, Cho JM, Chung KB, Han MC, Kim CW. Radiological findings of human fascioliasis. Abdom Imaging 1993; 18: 261-4.

7. Kabaalioglu A, Cubuk M, Senol U, Cevikol C, Karaali K, Apaydın A, et al Fascioliasis: US, CT, and MRI findings with new observations. Abdom Imaging 2000; 25: 400-4

8. Richter J, Freise S, Mull R, Millan JC. Fascioliasis: sonographic abnormalities of the biliary tract and evolution after treatment with triclabendazole. Trop Med Int Health 1999; 4: 774-81.

9. Kabaalioglu A, Apaydin A, Sindel T, Lüleci E. Fascioliasis: US-guided gallbladder aspiration: a new diagnostic methods for biliary fascioliasis. Eur Radiol 1999; 9: 880-2.

10. Markel EK, John DT, Krotoski WA. Immunodiagnostic techniques. In: Markel EK, John DT, Krotoski WA, editors. Markel and Woge's medical parasitology. Philadelphia: WB Saunders; 1999. p. 473-80.

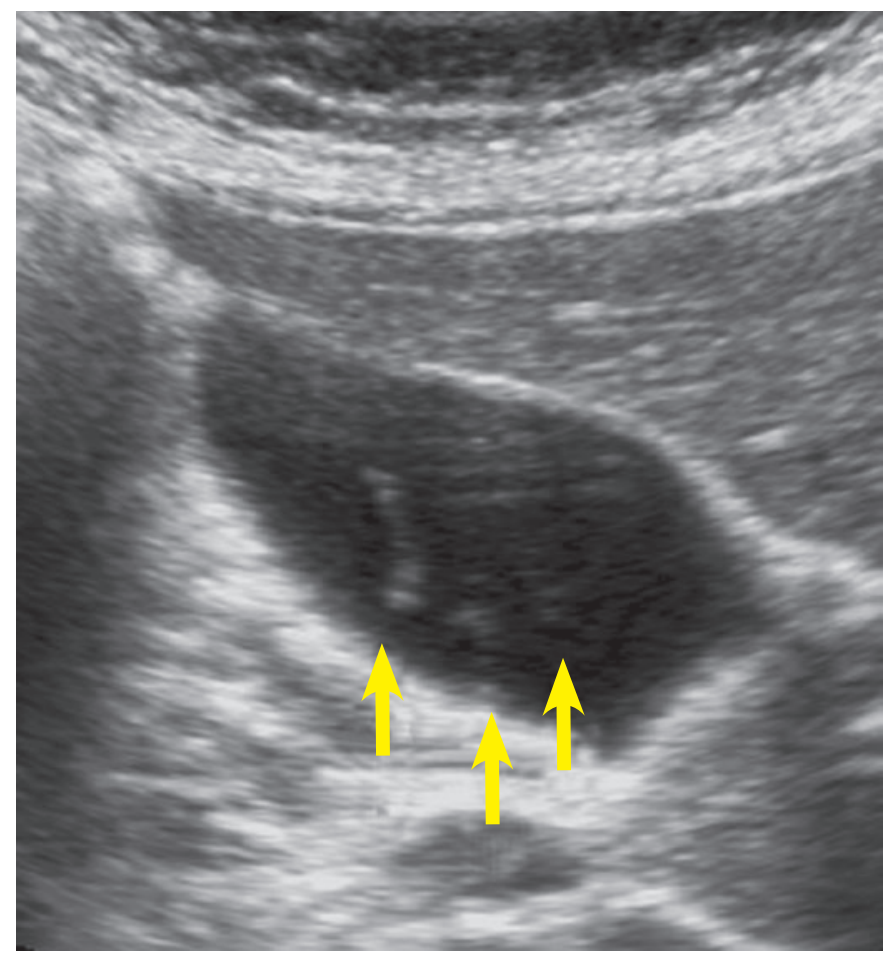

FIGURE 2A. Transverse sonogram shows floating echoes (arrows) with no acoustic shadowing in the gallbladder of patient with fascioliasis.

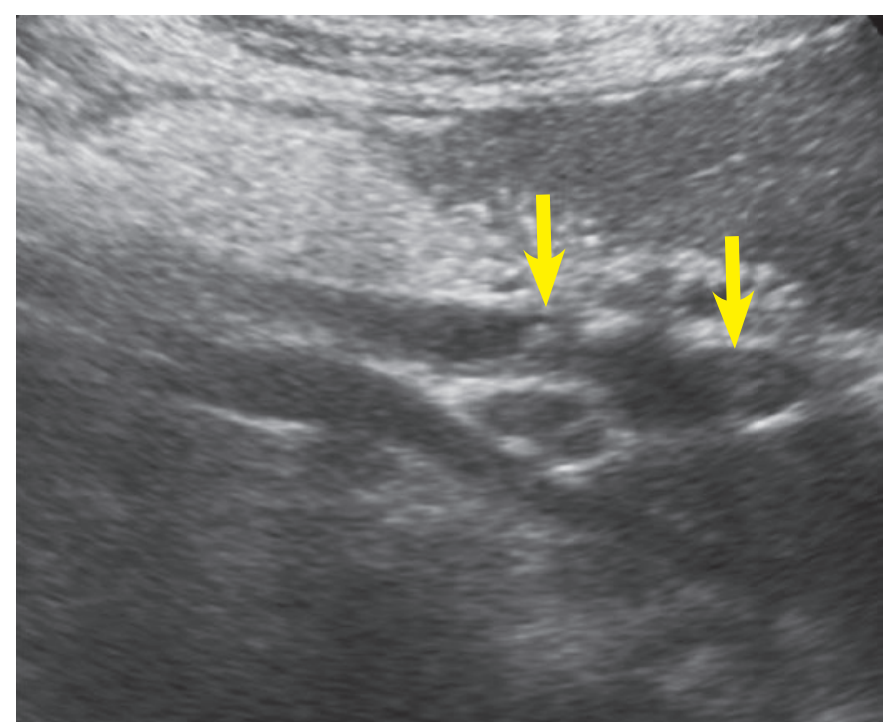

FIGURE 2B. Sonogram of the common bile duct depicts curvilinear echogenic parasites (arrow).

11. Koç Z, Ulusan S, Tokmak N. Hepatobiliary fascioliasis: imaging characteristics with a new finding. Diagn Interv Radiol 2009; 15: 247-51.

12. Ooms HWA, Puylaert JBCM, van der Werf SDJ. Biliary fascioliasis: US and endoscopic retrograde cholangiopancreatography findings. Eur Radiol 1995; 5:196-9. 


\title{
Development and characterization of a novel mAb against bilitranslocase - a new biomarker of renal carcinoma
}

\author{
Sendi Montanic ${ }^{1 *}$, Michela Terdoslavich ${ }^{2 *}$, Uros Rajcevic ${ }^{1}$, Luigina De Leo ${ }^{3}$, Serena Bonin ${ }^{4}$, \\ Vladka Curin Serbec ${ }^{1}$, Sabina Passamonti ${ }^{5}$ \\ ${ }^{1}$ Blood Transfusion Centre of Slovenia, Ljubljana, Slovenia \\ 2 Rottapharm Biotech S.r.l., Research Centre AREA Science Park, Trieste, Italy \\ 3 Institute of Child Health IRCCS “Burlo Garofolo" Trieste, Trieste, Italy \\ ${ }^{4}$ Department of Medical Sciences, University of Trieste, Cattinara Hospital, Trieste, Italy \\ ${ }^{5}$ Department of Life Sciences, University of Trieste, Trieste, Italy \\ Radiol Oncol 2013; 47(2): 128-137. \\ Received 18 December 2012 \\ Accepted 5 January 2013 \\ Correspondence to: Sendi Montanič, Blood Transfusion Centre of Slovenia, Šlajmerjeva 6, SI-1000, Ljubljana, Slovenia. \\ Phone: +386 1 5438220; E-mail: sendi.montanic@ztm.si \\ Disclosure: No potential conflicts of interest were disclosed. \\ * These authors contributed equally to this work.
}

Background. Bilitranslocase (TC 2.A.65.1.1) is a bilirubin-specific membrane transporter, found on absorptive (stomach and intestine) and excretory (kidney and liver) epithelia and in vascular endothelium. Polyclonal antibodies have been raised in rabbits in the past, using a synthetic peptide corresponding to AA65-77 of rat liver bilitranslocase, as an antigen. Affinity-purified antibodies from immune sera have been found to inhibit various membrane transport functions, including the bilirubin uptake into human hepatocytes and the uptake of some flavonoids into human vascular endothelial cells. It was described by means of immunohistochemistry using polyclonal antibodies that bilitranslocase expression is severely down-regulated in clear cell renal carcinoma. The aim of our work was development and characterization of high-affinity, specific mAbs against bilitranslocase, which can be used as a potential diagnostic tool in renal cell carcinoma as well as in a wide variety of biological assays on different human tissues.

Materials and methods. Mice were immunized with a multi-antigen peptide corresponding to segment 65-75 of predicted primary structure of the bilitranslocase protein. By a sequence of cloning, immune- and functional tests, we aimed at obtaining a specific monoclonal antibody which recognizes a 37 kDa membrane protein, and influences the transport activity of bilitranslocase.

Results. On the basis of previous results, specific IgM monoclonal antibodies were produced in BALB/C mice, in order to further improve and extend the immunological approach to the study of bilitranslocase in renal cancer cells as well as to develop its potential diagnostics use.

Conclusions. In this article we show an immunological approach, based on newly developed monoclonal antibodies, to a detailed biochemical and functional characterization of a protein whose gene and protein structure is still unknown. We were able to demonstrate our novel mAb as a tumor marker candidate of renal cell carcinoma, which may prove useful in the diagnostic procedures.

Key words: bilitranslocase; monoclonal antibody; peptide antigen; kidney; renal cell carcinoma; tumor marker

\section{Introduction}

Bilitranslocase (BTL, TC 2.A.65.1.1) is a plasma membrane transporter originally identified in rat liver. ${ }^{1}$ It transports various polyaromatic compounds, whether endogenous, such as bilirubin, or of plant origin, such as dietary flavonoids. It is expressed in various rat and human tissues, includ- 
ing the gastro-intestinal epithelium, the vascular endothelium ${ }^{2-4}$ and the kidney. ${ }^{5-7}$

Given the unusual feature of BTL coding sequence, which corresponds to the anti-sense strand of a segment of the gene encoding for the plasma protein ceruloplasmin ${ }^{1}$, the only way to assess the expression of this protein in cells and tissues is by immunological approach, using specific anti-peptide antibodies.

A previous investigation using anti-peptide polyclonal antibodies has shown that bilitranslocase expression is severely down-regulated in clear cell renal carcinoma. ${ }^{8}$

The most used anti-peptide, polyclonal antibody, targeting an extracellular domain of mammalian bilitranslocase was produced by immunizing rabbits with a multi-antigen peptide, corresponding to segment $65-75$ of the primary structure of rat liver bilitranslocase.

The affinity-purified antibody showed a wide biological activity, recognizing both the denatured protein in immunoblots and inhibiting its function in intact cells and purified plasma membrane fractions. ${ }^{9}$

To further improve and extend the immunological approach to the study of bilitranslocase expression in pathological tissue in kidney cancers, we aimed to produce a monoclonal antibody raised in $\mathrm{BALB} / \mathrm{c}$ mice that, while keeping the above biological features, would also meet the requirements of indefinite reproducibility, stability and long shelflife necessary for a reliable diagnostic tool.

Renal cell carcinoma (RCC), the most common neoplasm of the adult kidney accounts for $2-3 \%$ of all malignant diseases in adults. It is the seventh most common cancer in men and the ninth most common in women. Global incidence and mortality rates of RCC are rising ${ }^{10}$ with its incidence worldwide of about 209000 new cases per year and 102000 deaths per year. ${ }^{11}$ The majority of kidney tumors are of the clear cell (ccRCC) subtype. ${ }^{12}$ Although imaging techniques for abdominal screening have led to the increased incidental detection of renal tumors ${ }^{13}$, unfortunately $25-30 \%$ patients still have metastases at presentation. The prognosis of RCC is quite variable. The greatest risk of recurrence following nephrectomy is within the first 3-5 years. ${ }^{14}$ Metastatic renal cell carcinoma (RCC) is one of the most treatmentresistant malignancies and patients have a dismal prognosis with a $<10 \%$ five-year survival rate. The identification of markers that can predict the potential of metastases will have a great impact in improving the patient's outcome. ${ }^{15-17}$ Currently, the patient's prognosis is assessed by histological parameters and a multivariate analysis developed at Memorial Sloan Kettering ${ }^{18}$, but neither is sufficiently accurate. A more accurate assessment of the prognosis is urgently needed to better guide the patient's management. As our preliminary data on bilitranslocase expression in renal cancer compared to corresponding normal tissue showed remarkable differences, we aimed at producing a monoclonal antibody that would meet the requirement of serving as a tool for the assessment of bilitranslocase as a novel biomarker of human kidney cancers.

\section{Materials and methods}

\section{Experimental design}

Mice were immunized with a multi-antigen peptide corresponding to segment $65-75$ of predicted primary structure of the bilitranslocase protein. By a sequence of cloning, immune- and functional tests, we aimed at obtaining a specific IgM monoclonal antibody, which recognizes a $37 \mathrm{kDa}$ membrane protein, and influences the transport activity of BTL, by inhibiting the transfer of BSP in plasma membrane vesicles. Thus, we aimed at producing a tool suitable to give an insight into both the expression and the functioning of BTL.

\section{Peptide and peptide conjugate}

A multi-antigen peptide corresponding to segment 65-75 of the predicted amino acid sequence of bilitranslocase (AA 65-75; EDSQGQHLSSF) was conjugated to keyhole limpet hemocyanin (KLH) by Inject mcKLH Immunogen EDC Conjugation Kit (Pierce).

\section{Immunization of Balb/c mice and generation of mAbs}

Animals were fed with standard laboratory chow (Harlan Teklad) and tap water ad libitum; they were housed in temperature controlled rooms and humidity at the Animal House of the Blood Transfusion Centre of Slovenia (BTCS), Ljubljana, Slovenia. Three female BALB/c mice were immunized subcutaneously with $15 \mu \mathrm{g}$ of multi-antigen peptide in complete Freund's adjuvant followed by three intraperitoneal inoculations of $15 \mu \mathrm{g}$ of the same antigen in incomplete Freund's adjuvant after 2, 4 and 9 weeks (authorized by Veterinary Administration of the Republic of Slovenia, No.: 
3440-63/2006, of 31.7.2006). After $4^{\text {th }}$ immunization, polyclonal sera were collected from the tail vein and antibody titres were estimated with indirect ELISA (as described below). Two mice with the highest antibody titre were boosted with peptide in physiological saline and sacrificed. Fusion of spleen lymphocytes with mouse myeloma NS1 cells was performed by hybridoma technology, according to our protocols. After HAT (hypoxanthine-aminopterine-thymidine) selection, hybridomas producing antibodies of desired specificity were determined by indirect ELISA and cloned by limiting dilution.

\section{Screening of the sera and hybridoma supernatants by enzyme-linked immunosorbent assay (ELISA)}

The presence of specific antibodies in antisera and hybridoma supernatants was checked by indirect ELISA. Microtiter plates (Corning) were coated with $50 \mu \mathrm{l}$ of peptide $(3 \mu \mathrm{g} / \mathrm{ml})$ in $50 \mathrm{mM}$ carbonate/bicarbonate buffer ( $\mathrm{pH}$ 9.6), incubated overnight at $+4^{\circ} \mathrm{C}$, washed with PBS $0.05 \%$ Tween 20 , pH 7.2 (buffer B) and then blocked with 1\% BSA (bovine serum albumin) in the same buffer (buffer C). $50 \mu$ lof hybridoma supernatant or corresponding serum dilution was added to the wells and incubated for $90 \mathrm{~min}$ at $+37^{\circ} \mathrm{C}$. Negative isotypic controls were carried out by using equivalent amounts of hybridoma supernatants producing $\mathrm{mAbs}$ against blood group antigen $\mathrm{B}$. Plates were washed with buffer $B$ and $50 \mu l$ of HRP conjugated anti-mouse immunoglobulin (Jackson ImmunoResearch), diluted 1:3000 in buffer C, was added to each well and incubated for $90 \mathrm{~min}$ at $+37^{\circ} \mathrm{C}$. After a final wash in buffer B, $50 \mu \mathrm{l}$ ABTS substrate (Sigma) was added to each well. The absorbance was measured at $405 \mathrm{~nm}$ after $30 \mathrm{~min}$ of incubation at $+37^{\circ} \mathrm{C}$. Titres were evaluated by the end-point dilution approach (the highest dilution of the sera that was still two times higher as the background).

\section{Purification of monoclonal antibodies}

Monoclonal antibodies were purified from the cell culture supernatants by liquid chromatography system FPLC on HiTrap Protein G HP column (GE Healthcare) by affinity chromatography, using $0.1 \mathrm{M}$ glycine, $\mathrm{pH}$ 2.7, for elution or HiTrap IgM Purification HP column (GE Healthcare) by thiophilic adsorption on 2-mercaptopyridine, coupled to Sepharose at $1 \mathrm{M}$ ammonium sulphate.
Rat liver subfractions preparation: plasma membrane vesicles, microsome and cytosolic fraction

Subfractions were prepared from Wistar albino female rat liver $(250 \mathrm{~g})$. Animals were fed with standard laboratory chow (Harlan Teklad) and tap water ad libitum; they were housed in temperature controlled rooms and humidity at the Animal House of the University of Trieste according to the provision of the European Community Council Directive (n.86/609/CEE) and to the provision of Italy (D.L.vo 119/92). For the preparation of plasma membrane vesicles, microsomes and cytosolic fractions, 6 rats were used as described below.

\section{Plasma membrane vesicles preparation}

Plasma membrane vesicles were prepared from 3 rat livers as described. ${ }^{7}$ The final pellet was suspended in $10 \mathrm{mM}$ Hepes, $\mathrm{pH} 7.4 / 0.25 \mathrm{M}$ sucrose, and stored in liquid nitrogen. Vesicles, thawed at $+37^{\circ} \mathrm{C}$, were used for the bilitranslocase activity assay. When denaturized in SDS-PAGE sample buffer, they were subjected to the western blot analysis.

\section{Rat Liver Microsomes and cytosolic subfraction}

To get sufficient tissue preparation to perform all assays, three livers ( $7 \mathrm{~g}$ each) were rapidly excised, minced with scissors and washed out the excess of blood with chilled Homogenization Buffer (1 $\mathrm{mM}$ EDTA in PBS, pH 7.4). Liver pieces were further suspended in 4 volumes, equivalent to sample weight, of Homogenization Buffer with Protease Inhibitor Cocktail (PIC; Sigma) and homogenized by $30 \mathrm{ml}$ Glass/Teflon grinder, submerged in a small bucket of ice during homogenization. The homogenate was further centrifuged for $15 \mathrm{~min}$ at $15.000 \mathrm{~g}$ at $+4^{\circ} \mathrm{C}$. Mitochondrial pellet was discarded and the supernatant was centrifuged at 100.000 $\mathrm{g}$ for $60 \mathrm{~min}$ at $+4^{\circ} \mathrm{C}$. The upper lipid layer was removed, the cytosolic supernatant collected (SN2), whereas the microsome pellet was resuspended in Homogenisation Buffer with PIC (Sigma). Both samples (SN2 and microsomes) were aliquoted and stored at $-80^{\circ} \mathrm{C}$. Protein concentration was measured according to the Bradford method.

\section{Western blot}

Rat plasma membrane microsome and cytosolic preparations were denaturated with SDS-PAGE 
sample buffer at $+100^{\circ} \mathrm{C}$ for $5 \mathrm{~min} .50 \mu \mathrm{g}$ of sample per lane was loaded on SDS-tris-glycine polyacrilamide gels (9\%) and separated by PAGE. The separated proteins were transferred to $0.2 \mu \mathrm{m}$ nitrocellulose membranes (Whatman) by a standard procedure. The membranes were blocked in 5\% non-fat milk and incubated for $90 \mathrm{~min}$ at room temperature (RT) with tested hybridoma supernatants, diluted $1: 5$ in $1 \%$ milk/TBS-T, or purified monoclonal antibodies $(\mathrm{mAb})$ at a final concentration of $10 \mu \mathrm{g} / \mathrm{ml}$. As negative controls were used equivalent amounts of purified monoclonal antibody of the same isotype against blood group antigen B. Membranes were washed with TBS-T and incubated for 90 min at RT with HRP labeled anti-mouse secondary antibody (Jackson ImmunoResearch) 1:5000 in $1 \%$ milk/TBS-T. Chemiluminescence detection was performed using ECL reagent (Amersham Biosciences).

\section{Cell culture}

Human hepatoblastoma HepG2 cell line was obtained from American Type Culture Collection (Rockville, MD, USA) and maintained in Eagle's Minimum Essential Medium (Sigma), supplemented with $2 \mathrm{mM}$ glutamine, $1 \mathrm{mM}$ Sodium pyruvate, $10 \%(\mathrm{v} / \mathrm{v})$ fetal bovine serum, $100 \mu \mathrm{g} / \mathrm{ml}$ streptomycin and $100 \mathrm{U} / \mathrm{ml}$ penicillin (Sigma). Cells were grown in a humidified incubator at $5 \% \mathrm{CO}_{2}$ and $+37^{\circ} \mathrm{C}$. Cells for the flow-cytometry analysis were detached from the flasks after 6 days of culture with $20 \mathrm{mM}$ EDTA and used for the experiment. Alternatively, cells were seeded on cover glasses and after 4 days of culture, they were fixed with $2 \%$ paraformaldehyde, being ready for the immunocytochemistry assay.

\section{Flow cytometry}

For each sample, $5 \times 10^{5}$ HepG2 cells were first washed with staining solution (1\% FCS, $0.02 \%$ Na-azide in PBS), stained with primary $\mathrm{mAb}$ for 30 min at $+4^{\circ} \mathrm{C}$, then washed again with staining solution and incubated with secondary antibody (Sigma), diluted 1:50 for $30 \mathrm{~min}$ at $+4^{\circ} \mathrm{C}$. After staining, cells were washed three times and resuspended in $300 \mu \mathrm{l}$ of staining solution. Detection was immediately performed on a flow cytometer (Becton Dickinson). Polyclonal rabbit antibodies were used as a positive control. For the intracellular analysis, the cells were first fixed with $4 \%$ paraformaldehyde and permeabilised with permea- bilisation buffer $(0.5 \%$ saponine, $5 \%$ BSA, $0.02 \%$ Sodium Azide in PBS).

\section{Immunocytochemistry}

HepG2 cells, fixed on the cover glasses, were permeabilised with $0.2 \%$ Triton (Sigma) and blocked with blocking solution (5\% normal goat serum (Sigma) and 4\% BSA (Sigma) in PBS (Sigma) for 1 hour at $+37^{\circ} \mathrm{C}$. The staining with primary antibodies was performed at a final concentration of $50 \mu \mathrm{g} /$ $\mathrm{ml}$ overnight at $+4^{\circ} \mathrm{C}$. Negative controls were obtained by using purified monoclonal antibody of the same isotype against blood group antigen B. Next day, the cells were incubated with secondary antibodies (Sigma), diluted 1:100 in blocking solution for $1 \mathrm{~h}$ at $+37^{\circ} \mathrm{C}$. Cell nuclei were stained by incubating with $1 \mu \mathrm{g} / \mathrm{ml}$ DAPI (Sigma; 4-6-diamidino-2 phenylindole) in 1\% Tween-PBS for $5 \mathrm{~min}$ at RT. Between all incubation steps, cells were always washed with PBS. Finally, cells were dehydrated with $50 \%, 85 \%$ and $96 \%$ ethanol and mounted with 1.4-diazabicyclo (2.2.2.) octane (DABCO; Sigma) on microscope slides.

\section{Immunohistochemistry}

Immunohistochemistry (IHC) was performed on $3 \mu \mathrm{m}$ tissue sections of renal cancer carcinoma tissue section and a normal human kidney tissue section according to standard procedures. The immunostaining was performed with the Vectastain Universal Elite $A B C$ kit (Vector Laboratories). Briefly, after de-waxing and rehydration, slides were treated with $\mathrm{H}_{2} \mathrm{O}_{2}$ and blocked by Vectastain Elite $\mathrm{ABC}$ kit (Vector Laboratories) for $20 \mathrm{~min}$. Incubation with the primary antibody was performed at RT for 1 hour $(2 \mu \mathrm{g} / \mathrm{ml}$ in PBS containing $0.1 \%$ BSA). After washings in PBST, the slides were incubated for $30 \mathrm{~min}$ with the biotinylated secondary antibody and submitted to $A B C$ kit for the staining development. As described by the manufacturer, IHC was performed with DAB as chromogen and hematoxylin as counterstain. To investigate the specificity of the primary $\mathrm{mAb}$ in IHC detection, the primary antibody solution $(1 \mathrm{ml})$ was added on HepG2 monolayers grown on $75 \mathrm{~cm}^{2}$ flasks for $30 \mathrm{~min}$ at RT. Then, the same antibody solution was transferred to another flask of HepG2 cells monolayer and again incubated for 30 min. This procedure was repeated once more. This pre-adsorbed antibody solution was tested on tissue sections, where staining was negligible. 


\section{Bilitranslocase transport activity assay}

The transport activity of BTL was characterized in rat liver plasma membrane vesicles by real-time dual wavelength spectrophotometric recording of BSP (sulfobromophtalein) disappearance from the medium into the vesicles at $580 \mathrm{~nm}-514 \mathrm{~nm} .{ }^{19}$

\section{Electrogenic BSP uptake inhibition by antibodies}

The kinetics of bilitranslocase transport activity inhibition by antibodies was examined as described ${ }^{19}$ by pre-incubation of rat liver plasma membrane vesicles at $+37^{\circ} \mathrm{C}$ with antibody. Controls were carried out by using equivalent amounts of purified monoclonal antibody against blood group antigen B. Aliquots of the pre-incubation mixture were withdrawn during a $15 \mathrm{~min}$ span and added to the transport medium for the assay of bilitranslocase transport activity.

\section{Data analyses}

As previously described ${ }^{19}$, the extent of inactivation of each time sample was expressed as $\mathrm{T}_{\mathrm{I}} / \mathrm{T}_{0}$ (relative transport activity), where $\mathrm{T}_{0}$ and $\mathrm{T}_{\mathrm{I}}$ are transport activities, measured in the absence (or at time zero) or in the presence of the antibody, respectively. The following equation was fitted to data: $y=y_{0}+a e^{-k t}$, where $y=\mathrm{T}_{\mathrm{I}} / \mathrm{T}_{0}, y_{0}=$ residual $\mathrm{T}_{\mathrm{I}} /$ $\mathrm{T}_{0}$ at steady state, $a=1-y_{0}, e=2.7183, t=$ time and $k=$ inactivation rate constant $\left(\mathrm{min}^{-1}\right)$. Thus, the inactivation rate constants parameters in the absence $\left(k_{0}\right)$ and in the presence of increasing bilirubin concentrations (kS1, kS2, ... kSn) were obtained. The inhibition rate constants can be related to bilirubin concentration by the Scrutton \& Utter equation ${ }^{20}$

$\mathrm{kS} / \mathrm{k} 0=\mathrm{k} 2 / \mathrm{k} 1+\mathrm{Kd}(1-(\mathrm{kA} / \mathrm{k} 0)) /(\mathrm{S})$

where $\mathrm{kS}$ and $\mathrm{k} 0$ are the inactivation rate constants either in the presence or in the absence of various concentrations of a bilirubin, $\mathrm{k} 2$ and $\mathrm{k} 1$ are the rate constants of the inhibition of the bilitranslocase-bilirubin complex and of free bilitranslocase, respectively. $\mathrm{Kd}$ is the dissociation constant of the apparent bilitranslocase-bilirubin complex.

Data for the characterization of the kinetics of electrogenic BSP uptake fitted the MichaelisMenten equation and the apparent $\mathrm{Km}$ and Vmax values were derived with their standard errors. Data were analyzed by means of SigmaPlot 2001 (SPSS Science Software Gmbh).

\section{Statistics}

Immunoblotting experiments were performed 3 times, obtaining consistent results. Figure 1A displays the most successful one (less background, optimal protein load, optimal primary and secondary antibody dilutions and time of exposure).

The transport activity assay was performed on a single vesicle preparation. Tests were done in quintuplicate in preliminary experiments (not shown), testing the dependence of BSP uptake rate on either protein or valinomycin. The coefficients of variations were always below $2 \%$. All measurements, displayed in Figure 3A, B, C, are single. To assess the reproducibility of the parameters of the best fitting curves, experiments were performed at least three times.

\section{Results}

According to our previous results, the immunogenic epitope of interest is segment 65-75; EDSQGQHLSSF of the predicted primary structure of bilitranslocase. Polyclonal antibodies against such epitope already showed multiple biological activities, i.e. to react with 1) the protein, expressed in $E$. coli, 2) a single protein in rat liver microsomes, separated by SDS-PAGE electrophoresis and 3) the purified rat liver bilitranslocase. ${ }^{13}$ The same antibodies also inhibited a) the BSP electrogenic uptake in rat liver plasma membrane vesicles, $b$ ) the BSP electrogenic uptake in microsomes obtained from HepG2 cells and c) the uptake of bilirubin and BSP into HepG2 cells., ${ }^{9,21}$ These properties justified the development of a mAb raised against the same peptide in order to produce a reproducible and unlimited tool.

\section{Testing of the immune response against peptide, preliminary screening and cloning}

The polyclonal immune response to peptide EDSQGQHLSSF was tested by ELISA. Immunization resulted in a strong immune response in all injected $\mathrm{BALB} / \mathrm{c}$ mice. The mouse with the highest anti-peptide titre was chosen for the cell fusion. Two separate cell fusions of mouse splenocytes with NS1 mouse myeloma cells were performed.

Over 3000 hybridomas were obtained after the cell fusion and, after screening by ELISA, 67 clones produced antibodies, which reacted with the chosen peptide, but not with KLH. The second step of 
the selection was done by ELISA, using rat liver microsomes as antigen for coating the plate. The results restricted the number of positive clones to 22. The antibodies, produced by selected hybridoma cell lines, were also analyzed in western blot (WB), checking their binding capacity to rat liver microsome (M) or cytosolic fraction (SN2) denatured preparations (data not shown).

Selected hybridomas, which also satisfied cell culture freezing, growing features and production of stable mAbs, were chosen for cloning and additional screening. The first step of the screening confirmed the results, obtained before cloning of hybridomas by repeating ELISA and WB analysis. Fluorescence-activated cell sorting (FACS) and immunocytochemistry (ICC) were carried out as in vitro assays for the further antibody characterization. Selected antibodies recognized a protein with MW $37 \mathrm{kDa}$, both in microsomes rat liver and cytosolic preparations (data not shown). Clone 6E4/1F2 was the best candidate in our early selection criteria.

\section{Final screening, antibody characterization and applications}

Cell line 6E4/1F2 was further cloned and mAbs were purified. All ELISA/WB/FACS/ICC tests were repeated as described above and lead to the finally selected $\mathrm{mAb}$, named $6 \mathrm{E} 4 / 1 \mathrm{~F} 2 / 1 \mathrm{E} 2$, produced by stable hybridoma cell line.

WB analysis, performed with purified $\mathrm{mAb}$, as shown in Figure 1A, confirmed the binding to a protein with $\mathrm{MW}$ around $37 \mathrm{kDa}$. The purified mAb 6E4/1F2/1E2 was tested also in immunocytochemistry (Figure 1B) and as it is shown in this figure, the antibody formed immune complexes on the surface of fixed HepG2 cells.

Our findings show that the selected $\mathrm{mAb}$ displays the required features of selectivity and specificity of binding to BTL.

FACS analyses were carried out including both, intracellular and surface protocols. Fixed HepG2 cells were used only for intracellular staining, whereas surface staining was applied on non-fixed cells in order to limit any possible antigen damage due to the fixation procedure. This strategy was applied in order to confirm the apparent BTL localization, derived from ICC results. Figure 2 shows, as expected, prevalent extracellular staining.

The antibody was also tested for its inhibition of electrogenic bromosulphalein (BSP) transport in rat liver plasma membrane vesicles, a specific assay of bilitranslocase transport activity. Inhibition was time-dependent (Figure 3A) and linearly de-

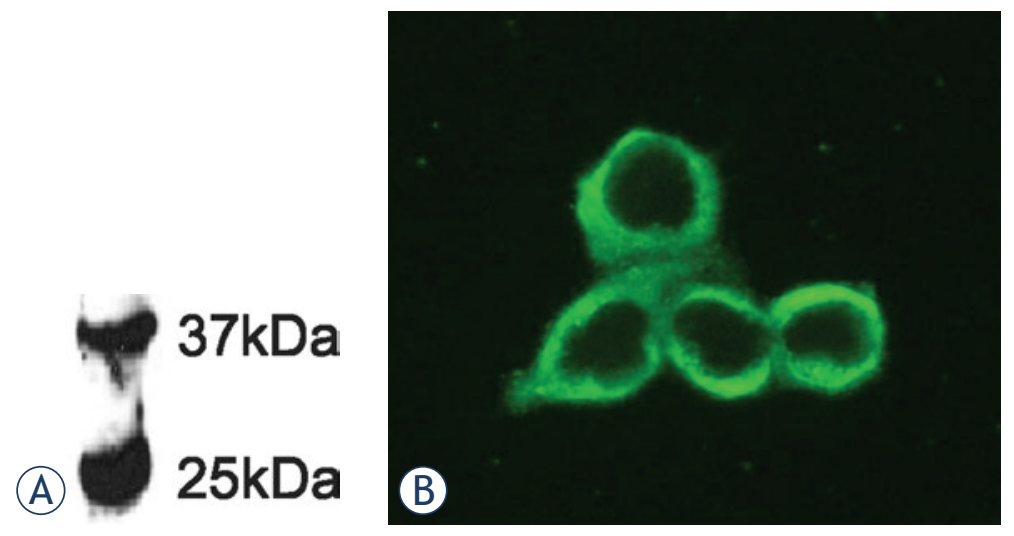

FIGURE 1. Application of purified anti-BTL $m A b$ in WB and ICC assays. (A) WB of rat liver denatured microsome (M), performed with purified mAb 6E4/1F2/1E2 (10 $\mu \mathrm{g} / \mathrm{ml}$ ). (B) ICC staining of HepG2 cells with mAb 6E4/1F2/1E2 (10 $\mu \mathrm{g} / \mathrm{ml})$.

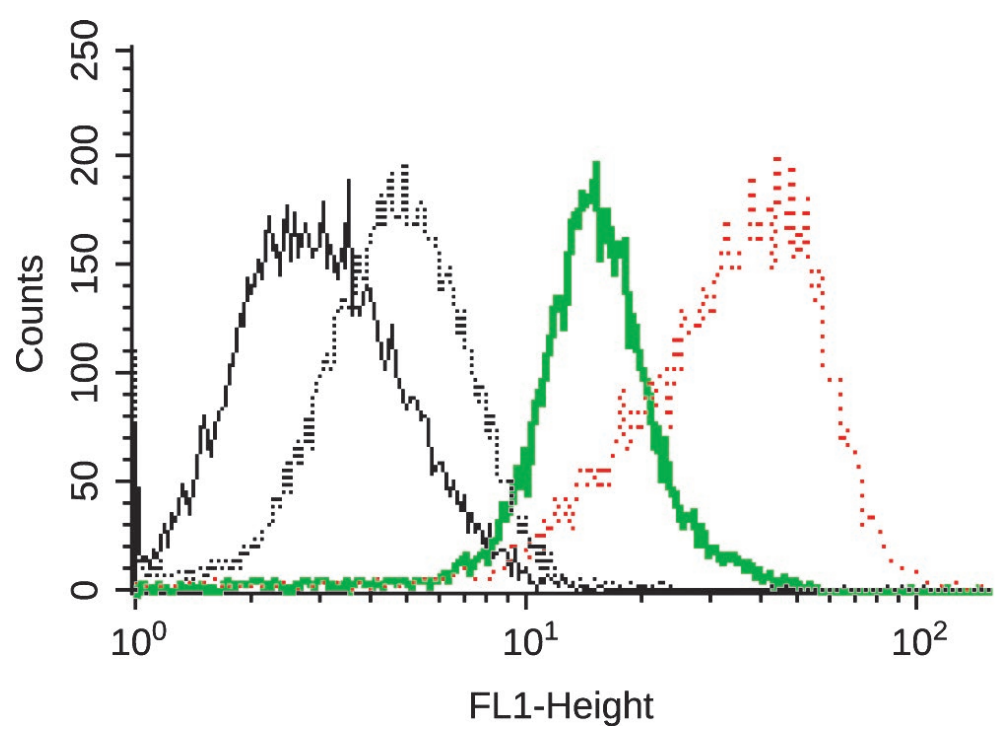

FIGURE 2. Application of purified anti-BTL mAb in FACS. Reactivity of mAb 6E4/1F2/1E2 with native BTL, expressed on HepG2 cells, determined by FACS as follows: cells only (black line), cells with secondary antibody (black dotted line), intracellular staining (green line), surface staining (red dotted line).

pendent on antibody concentration in the range tested (Figure 3A, inset). Two other clones of the cell line $6 \mathrm{E} 4 / 1 \mathrm{~F} 2$, i.e. $1 \mathrm{C} 8$ and $2 \mathrm{~A} 8$, were also included in testing. Clone 2A 8 was inactive. Clone $1 \mathrm{C} 8$ displayed a lower inhibition capacity than 1E2, the second order rate constant of inhibition of the latter being 1.4-fold higher than the former.

To check if the apparent transport inhibition observed might be due to an unspecific change of vesicles conductivity, the following experiment was carried out.

Vesicles $(2.4 \mathrm{mg} / \mathrm{ml}$ in $0.25 \mathrm{M}$ sucrose and 10 $\mathrm{mM}$ Hepes $\mathrm{pH}$ 7.4) were incubated with the anti- 

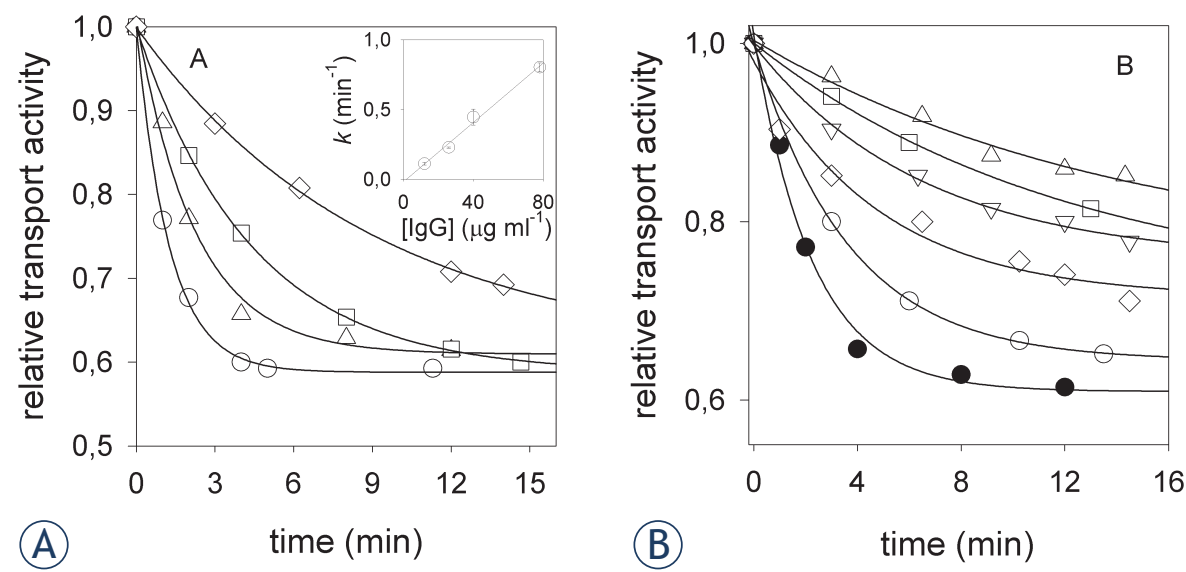

FIGURE 3. Application of purified anti-BTL mAb in protein activity inhibition. (A) Time-dependent inhibition of electrogenic BSP transport activity in rat liver plasma membrane vesicles by mAb 6E4/1F2/1E2. Vesicles (3.6 mg protein/ml) were pre-incubated with mAb $6 \mathrm{E} 4 / 1 \mathrm{~F} 2 / 1 \mathrm{E} 2$ ( $\mu \mathrm{g} / \mathrm{ml})$ as follows: 12 ( $\diamond)$, $26(\square), 40(\triangle), 78(O)$ at $37^{\circ} \mathrm{C}$. Aliquots $(3.5 \mu \mathrm{l})$ were assayed for electrogenic BSP transport (24 $\mu \mathrm{M}$ BSP). The calculated second order rate $\mathrm{k}$ was $0.01 \pm 0.00 \mathrm{~min}-1 \mathrm{ml} \mu \mathrm{g}-1 ; \mathrm{r} 2=0.99$. (B) Time-dependent inhibition of electrogenic BSP transport activity in rat liver plasma membrane vesicles by mAb

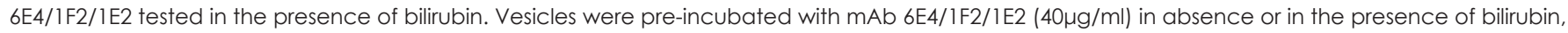
as follows $(\mathrm{nM}): 2(O), 5(\diamond), 12.5(\nabla), 30(\square), 50(\triangle)$ at $37^{\circ} \mathrm{C}$. Aliquots $(3.5 \mu \mathrm{ll}$ ) were assayed for electrogenic BSP transport $(24 \mu M \mathrm{BSP})$. (C) Scrutton \& Utter plot. The parameters of the regression curve are: $y_{0}=0.143 \pm 0.04 ;$ slope $=K_{d}=2.67 \pm 0.38 \mathrm{nM} ; r^{2}=0.94$. Best-fitting curves to the data were obtained as described in Materials and methods.

bodies $(12 \mu \mathrm{g} / \mathrm{ml})$ at $+37^{\circ} \mathrm{C}$ for $30 \mathrm{~min}$. Then, they were diluted twice in $0.15 \mathrm{M}$ potassium phosphate buffer $\mathrm{pH} 8.0$ at $+20^{\circ} \mathrm{C}$ and assayed for the electrogenic BSP uptake immediately after dilution and then after 1, 2 and $4 \mathrm{~min}$. In case of disruption of the membrane conductivity, it should be expected that $\mathrm{K}^{+}$would move from the medium into the vesicular compartment, whereas $\mathrm{H}^{+}$would move out from the vesicles ( $\mathrm{pH}$ 7.4) to the medium ( $\mathrm{pH}$ 8.0). So, the electrogenic BSP transport activity should be abolished, due to the collapse of the driving forces of BSP movement and accumulation into the vesicles, i.e. $\mathrm{K}^{+}$diffusion membrane potential and $\mathrm{pH} .{ }^{11}$ It was found that the transport activity was stable for 2 min following the addition of the potassium phosphate buffer $\mathrm{pH} 8.0(0.68 \pm 0.01$ of control) and decreased insignificantly at $4 \mathrm{~min}$ (from $0.68 \pm 0.01$ to $0.66 \pm 0.02$ ). This set of results indicates that the antibody changed neither the $\mathrm{K}^{+}$nor the $\mathrm{H}^{+}$ conductivity. Otherwise, a drastic and instantaneous effect of the assay should have occurred, i.e. a significant decrease of the transport rate.

To further assess if the antibody targeted a bilirubin-binding epitope, exposed on the surface on plasma membrane vesicles, inhibition tests were carried out in the presence of increasing concentrations of bilirubin, in the range $0 \mathrm{nM}-50 \mathrm{nM}$. As previously observed, bilirubin caused the inhibition reaction to proceed at a slower rate (Figure 3B,C). The rearrangement of the data by the Scrutton \& Utter equation enabled the derivation of the dis- sociation constant of the transporter-bilirubin complex $\left(\mathrm{K}_{\mathrm{d}}=2.67 \pm 0.38 \mathrm{nM}\right)$, which was similar to the one, previously obtained with polyclonal antisequence antibodies. It might be argued that the slower inactivation rate, observed in the presence of bilirubin might be ascribed to an interaction of the latter with the hypervariable region of the immunoglobulins, thus preventing antibody complexation with the protein epitope. A control experiment was, therefore, carried out to test this hypothesis. The antibody and bilirubin $(50 \mathrm{nM})$ were pre-incubated at $+37^{\circ} \mathrm{C}$ and then used to test the rate of BSP transport inactivation. No significant change of the rate constant could be observed (data not shown). However, the large decrease in the pseudo-first order rate constant of transport inactivation observed (dropping from $0.4456 \mathrm{~min}^{-1}$ in the absence of bilirubin to $0.0695 \mathrm{~min}^{-1}$ with $50 \mathrm{nM}$ bilirubin) cannot be accounted for by a sequestration of the antibody by bilirubin, since, under the prevailing experimental conditions, $\mathrm{mAb}$ was in 5 -fold molar or 10-fold equivalent excess, respectively, in favor of the highest bilirubin concentration tested, i.e. 50 $\mathrm{nM}$. Even by assuming that bilirubin complexed with the antibody so reducing its effective concentration by an over-estimated $20 \%$, this should be revealed by a decrease of the pseudo-first order rate constant of transport inactivation from ca. 0.45 $\mathrm{min}^{-1}$ to ca. $0.35 \mathrm{~min}^{-1}$, as predicted by the value of the second-order rate constant obtained from the experiment shown in Figure 3. However, $50 \mathrm{nM}$ 

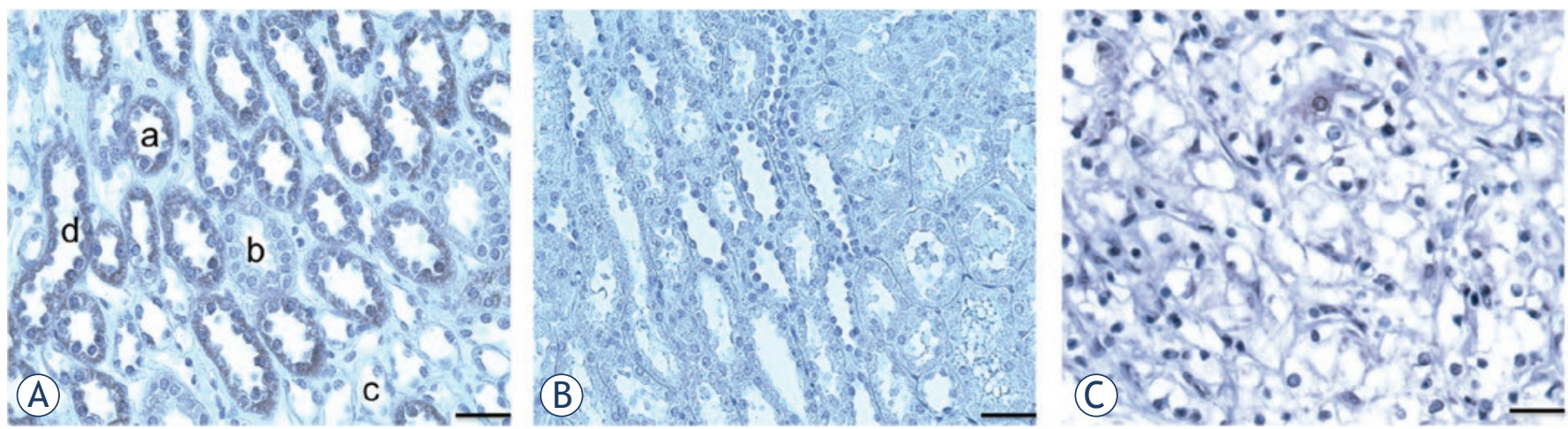

FIGURE 4. Application of purified anti-BTL mAb in IHC on human RCC and normal kidney tissue sections.

bilirubin caused a 5-fold higher decrease of the first-order rate constant (i.e. $0.0695 \mathrm{~min}^{-1}$ ).

The use of this antibody in immunohistochemistry was tested on human early stage (grade I.) RCC and on normal kidney. Figure $4 \mathrm{~A}$ shows that the antibody selectively stained distal tubules and the collecting tubules, but none of the segments of the Henle loop. The proximal tubules were also positive (not shown), whereas the vascular structures were negative (not shown). Significantly, staining was nearly abolished (Figure 4B) by pre-adsorption of the antibody solution on a monolayer of HepG2 cells. This outcome was expected, since the immunogenic peptide is a part of an extra-cellular domain of hepatic BTL (Figure 2). ${ }^{9}$ On the opposite, the staining of the grade I RCC tissue section (Figure 4C) was negative.

\section{Discussion}

\section{Bioactivity of the bilitranslocase mAb}

Data obtained by numerous immunological methods show that we selected an IgM mAb, specific for a bilirubin-binding membrane protein. This $\mathrm{mAb}$ upgraded properties of our previously tested polyclonal antibodies, with unlimited source and monospecific binding, binding not only unfolded protein, but also a native one, where it causes a functional loss (Figure 3). Such noteworthy property depends on the fact that antibody binds to an extracellular epitope involved in the transport cycle of the protein. Thus, antibodies targeting that epitope enable to follow not just the expression but also the function of bilitranslocase in viable experimental models, endowed with increasing morphologic complexity, as best seen in vascular endothelial cells. ${ }^{2,4}$

Such dual activity of the mAb is particularly advantageous, since bilitranslocase antibodies are currently the only probes to study the occurrence and the function of bilitranslocase. This transporter is, indeed, encoded by an antisense transcript, disabling its detection by current molecular biology tools and approaches. As a consequence, sense-antisense overlaps limit the choice of primers and probes and antisense RNA has usually very low expression levels. ${ }^{22}$ The coding sequence of bilitranslocase (GenBank: Y12178.1) is $94 \%$ homologous to a segment of the antisense strand of the ceruloplasmin transcript, including part of its 3'-UTR (NM_012532, segment 2621-3637). The bilitranslocase-ceruloplasmin sense-antisense pair is classified as entry RNO 30106 in the anti-CODE database (http://www.anticode.org) that collects these RNAs. Antisense RNAs are usually non-coding RNAs, though the bilitranslocase transcript has mRNA-like characteristics. ${ }^{1,21}$

In cell biology, the correlation between downregulation of the expression of a protein, by either RNA interference or gene deletion, and decreased cellular function, represents an essential piece of evidence. However, with proteins, translated from antisense transcripts, this cannot be accomplished by the currently validated approaches. Thus, it is clear that antibodies, especially if endowed with the abovementioned dual properties, still represent the reagents of election to track and functionally characterize the so far rare proteins, translated from antisense transcripts.

\section{Bilitranslocase as a negative tumor marker}

Our preliminary findings by immunohistochemical tests showed that bilitranslocase was severely down-regulated in grade I clear cell kidney cancer (cc RCC) compared to normal kidney tissue. The reason for such difference is currently unknown. 
Further investigations are needed to verify if it is associated to this renal cancer specific histological type and/or to renal cancer aggressiveness. Anyway, bilitranslocase could be inactivated in cancerous tissue for various reasons. This may point at bilitranslocase involvement in tumor suppressor activity and thus its inactivation potentially contributing to tumorigenesis in kidney tissue. Further analyses are needed to confirm this hypothesis.

\section{Further Applications of the anti-bilitranslocase mAb}

\section{Modulation of expression}

The mAb will be an invaluable tool for the study of the expression of bilitranslocase in various stages of rat embryogenesis and cell differentiation. In the intestinal epithelium, the onset of bilitranslocase expression appears to coincide with the differentiation of stem cells into mature epithelial cells. ${ }^{1}$ This observation would justify the employment of bilitranslocase mAb to mark the stages of normal differentiation as well as of de-differentiation in carcinogenesis.

\section{Tissue and cell distribution of bilitranslocase and its assessment as a membrane transporter}

Immunological assays allow the identification of the presence of bilitranslocase in tissues and cells, in both animal and plant species, helping to infer aspects of organ physiology depending on the bilitranslocase function. The expression of bilitranslocase in both absorptive (gastro-intestinal) and excretory (liver and kidney) epithelia as well as in the vascular endothelium makes it a tremendously interesting drug target. As shown above, bilitranslocase is assessed in plasma membrane vesicles as a BSP electrogenic transporter. ${ }^{19}$ This activity is competitively inhibited by various compounds, suggesting that they cannot only bind into the transport site of the protein, but also be directly transported. The latter demonstration can be obtained in isolated cultured cells, where the uptake of a given molecule is inhibited by cells pre-treatment with bilitranslocase antibodies. ${ }^{2,9}$ This antibody-based approach to the study of drug transport can be implemented in normal cells and tissues, without gene or RNA manipulation, which unbalance the transcription level of a protein, or counterbalance on the expression level of function- ally subsidiary proteins, or disrupt the intracellular network of protein-protein interactions. For these features, the immunological approach to the study of other drug and nutrient transporters might become standard.

\section{Conclusions}

In conclusion, the described $\mathrm{mAb}$ might be used as a research tool to assess bilitranslocase as a marker of transition from normal tissue to its neoplastic transformation in human kidney. In further studies, this antibody could be developed as a diagnostic reagent for kidney cancer. However, this $\mathrm{mAb}$ will enable us to screen a variety of tissue samples to prove its use in diagnostics.

\section{Authors' contributions}

MT, SM, LD, SB and UR participated in the design of the study, carried out cell fusions, cell culturing, immune- and functional tests, acquisition, analysis and interpretation of data and manuscript drafting. SP and VČŠ have made contributions to conception, coordination and design of the study, drafting the manuscript and critical revisions. All authors read and approved the final manuscript.

\section{Acknowledgements}

We thank Prof. Giorgio Stanta (University of Trieste) for support in IHC tests, dr. Federica Tramer (University of Trieste) for providing Figure 1A, dr. Miha Kosmač, BTCS for the help with figure preparation, dr. Adriano Savoini (T\&B Associati, Area Science Park, Trieste, Italy) for his help in framing the Discussion, based on a previous grant application (ERC-2010-AdG_20100317), Melita Gracar and Marjana Šprohar, BTCS for technical assistance.

The financial support by the following bodies is acknowledged: University of Trieste (co-funding of a post-doctoral fellowship to M.T.), Regione Friuli Venezia Giulia (Progetto GISVI, L.R. 26/2010), Ministero degli Affari Esteri (Cooperazione scientifica e tecnologica Italia-Slovenia 2006-2009) and European Regional Development Fund (Crossborder cooperation Programme Italy-Slovenia 2007-2013, Trans2care strategic project). 


\section{References}

1. Passamonti S, Terdoslavich M, Franca R, Vanzo A, Tramer F, Braidot E, et al. Bioavailability of flavonoids: a review of their membrane transport and the function of bilitranslocase in animal and plant organisms. Curr Drug Metab 2009; 10: 369-94.

2. Maestro A, Terdoslavich M, Vanzo A, Kuku A, Tramer F, Nicolin V, et al. Expression of bilitranslocase in the vascular endothelium and its function as a flavonoid transporter. Cardiovasc Res 2010; 85: 175-83.

3. Ziberna L, Tramer F, Moze S, Vrhovsek U, Mattivi F, Passamonti S. Transport and bioactivity of cyanidin 3-glucoside into the vascular endothelium. Free Radic Biol Med 2012; 52: 1750-9.

4. Ziberna L, Lunder M, Tramer F, Drevensek G, Passamonti S. The endothelial plasma membrane transporter bilitranslocase mediates rat aortic vasodilation induced by anthocyanins. Nutr Metab Cardiovasc Dis 2013; 23: 68-74.

5. Elias MM, Lunazzi GC, Passamonti S, Gazzin B, Miccio M, Stanta G, et al. Bilitranslocase localization and function in basolateral plasma membrane of renal proximal tubule in rat. Am J Physiol 1990; 259: 559-64.

6. Vanzo A, Terdoslavich M, Brandoni A, Torres AM, Vrhovsek U, Passamonti S. Uptake of grape anthocyanins into the rat kidney and the involvement of bilitranslocase. Mol Nutr Food Res 2008; 52: 1106-16.

7. Brandoni A, Di Giusto G, Franca R, Passamonti S, Torres AM. Expression of kidney and liver bilitranslocase in response to acute biliary obstruction. Nephron Physiol 2010; 114: 35-40.

8. Golijanin DJ, Marshall J, Cardin A, Singer EA, Wood RW, Reeder JE, et al. Bilitranslocase (BTL) is immunolocalised in proximal and distal renal tubules and absent in renal cortical tumors accurately corresponding to intraoperative near infrared fluorescence (nirf) expression of renal cortical tumors using intravenous indocyanine green (ICG). [Abstract]. J Urol 2008; 179(4 Suppl S): 137.

9. Passamonti S, Terdoslavich M, Margon A, Cocolo A, Medic N, et al. Uptake of bilirubin into HepG2 cells assayed by thermal lens spectroscopy. Febs $J$ 2005; 272: 5522-35.

10. Hollingsworth JM, Miller DC, Daignault S, Hollenbeck BK. Rising incidence of small renal masses: a need to reassess treatment effect. J Natl Cancer Inst 2006; 98: 1331-4.

11. Gupta K, Miller JD, Li JZ, Russell MW, Charbonneau C. Epidemiologic and socioeconomic burden of metastatic renal cell carcinoma (mRCC): a literature review. Cancer Treat Rev 2008; 34: 193-205.

12. Cheville JC, Lohse $\mathrm{CM}$, Zincke $\mathrm{H}$, Weaver AL, Leibovich BC, Frank I, et al. Sarcomatoid renal cell carcinoma: an examination of underlying histologic subtype and an analysis of associations with patient outcome. Am J Surg Pathol 2004; 28: 435-41.

13. Russo P. Renal cell carcinoma: clinical features and management. Methods Mol Med 2001; 53: 3-33.

14. John S Lam, Tobias Klatte, Alberto Breda. Staging of renal cell carcinoma: Current concepts. Indian J Urol 2009; 25: 446-54.

15. Masui O, White NM, Desouza LV, Krakovska O, Matta A, Metias S, et al. Quantitative proteomic analysis in metastatic renal cell carcinoma reveals a unique set of proteins with potential prognostic significance. $\mathrm{Mol} \mathrm{Cell}$ Proteomics 2012 Oct 17. 2013; 12: 132-44..

16. Rajer M. Kidney cancer. Radiol Oncol 2007; 41: 64-71.

17. Linnert $\mathrm{M}$, Iversen $\mathrm{H}$, Gehl J. Multiple brain metastases - current management and perspectives for treatment with electrochemotherapy. Radiol Oncol 2012; 46: 271-8.

18. Motzer RJ, Mazumdar M, Bacik J, Berg W, Amsterdam A, Ferrara J. Survival and prognostic stratification of 670 patients with advanced renal cell carcinoma. J Clin Oncol 1999; 17: 2530-40.

19. Passamonti S, Tramer F, Petrussa E, Braidot E, Vianello A. Electrogenic bromosulfalein transport in isolated membrane vesicles: implementation in both animal and plant preparations for the study of flavonoid transporters. In: Fett-Neto AG editor. Plant Secondary Metabolism Engineering, Methods in molecular biology. Totowa: Humana Press Inc; 2010. pp. 307-36.

20. Scrutton MC, Utter MF. Pyruvate carboxylase. V. Interaction of the enzyme with adenosine triphosphate. J Biol Chem 1965; 240: 3714-23.
21. Passamonti S, Cocolo A, Braidot E, Petrussa E, Peresson C, Medic N, et al. Characterization of electrogenic bromosulfophthalein transport in carnation petal microsomes and its inhibition by antibodies against bilitranslocase. Febs J 2005; 272: 3282-96.

22. Werner A, Berdal A. Natural antisense transcripts: sound or silence? Physiol Genomics 2005; 23: 125-31. 


\title{
The correlation between the levels of tissue inhibitor of metalloproteinases 1 in plasma and tumour response and survival after preoperative radiochemotherapy in patients with rectal cancer
}

\author{
Irena Oblak¹, Vaneja Velenik¹, Franc Anderluh¹, Barbara Mozina², Janja Ocvirk \\ ${ }^{1}$ Department of Radiotherapy, Institute of Oncology Ljubljana, Ljubljana, Slovenia \\ 2 Department of Laboratory Diagnostics, Institute of Oncology Ljubljana, Ljubljana, Slovenia \\ ${ }^{3}$ Department of Medical Oncology, Institute of Oncology Ljubljana, Ljubljana, Slovenia
}

Radiol Oncol 2013; 47(2): 138-144.

Received 18 January 2013

Accepted 3 February 2013

Disclosure: No potential conflicts of interest were disclosed.

Correspondence to: Assist. Prof. Irena Oblak, MD, PhD, Department of Radiotherapy, Institute of Oncology Ljubljana, Zaloška 2, SI-1000 Ljubljana, Slovenia. Phone: +386 15879 661; Fax: +386 15879 304; E-mail: ioblak@onko-i.si

Background. The aim of this study was to analyse whether the level of tissue inhibitor of metalloproteinases (TIMP) 1 is associated with the tumour response and survival to preoperative radiochemotherapy in rectal cancer patients. Patients and methods. Ninety-two patients with histologically confirmed non-metastatic rectal cancer of clinical stage I- III were treated with preoperative radiochemotherapy, surgery and postoperative chemotherapy. Plasma TIMP-1 concentrations were measured prior to the start of the treatment with an enzyme-linked immunosorbent assay (ELISA). Results. Median follow-up time was 68 months (range: 3-93 months) while in survivors it was 80 months (range: 68-93 months). The 5-year locoregional control (LRC), disease-free survival (DFS), disease-specific survival (DSS) and overall survival (OS) rates for all patients were $80.2 \%, 56.4 \%, 63.7 \%$ and $52.2 \%$, respectively. The median TIMP- 1 level was $185 \mathrm{ng} / \mathrm{mL}$ (range: $22-523 \mathrm{ng} / \mathrm{mL}$ ) and the mean level ( \pm standard deviation) was 192 ( \pm 87 ) ng/mL. Serum TIMP-1 levels were found to be significantly increased in patients with preoperative CRP $>12 \mathrm{mg} / \mathrm{L}$ and in those who died from rectal cancer or had CT4 tumours. No correlation was established for age, gender, carcinoembriogenic antigene (CEA) level, platelets count, histopathological grade, response to preoperative therapy, resectability and disease reappearance. On univariate analysis, various parameters favourably influenced one or more survival endpoints: TIMP-1 $<170 \mathrm{ng} / \mathrm{mL}, \mathrm{CRP}<12 \mathrm{mg} / \mathrm{L}$, platelets count <290 10E9/L, CEA <3.4mg/L, age <69 years, male gender, early stage disease (CNO and/or CT2-3), radical surgery (RO) and response to preoperative radiochemotherapy. In multivariate model, LRC was favourably influenced by N-downstage, DFS by lower CRP and N-downstage, DSS by lower CRP and $\mathrm{N}$-downstage and OS by lower TIMP-1 level, lower CRP and N-downstage.

Conclusions. Although we did not find any association between pretreatment serum TIMP-1 levels and primary tumour response to preoperative radiochemotherapy in our cohort of patients with rectal cancer, TIMP-1 levels were recognized as an independent prognostic factor for OS in these patients.

Key words: tissue inhibitor of metalloproteinases; rectal cancer; preoperative radiochemotherapy; prognostic factors

\section{Introduction}

Rectal cancer is one of the major health problems due to increasing incidence and in a substantial proportion of patients it is diagnosed in advanced stages. The primary treatment is surgery. In transmural extension of tumour or node positive disease, preoperative radiochemotherapy is recommended to improve local control and survival. ${ }^{1-3}$ 
Poor prognosis of rectal cancer patients was attributed to the increase in tumour aggressiveness and its metastatic potential which, among others, were found to be determined by the activity of matrix metalloproteinases (MMPs) and their inhibitors (TIMPs) which play an important role in the process of degradation of the extracellular matrix and the basal membranes. The activity of the MMPs depends on the balance between the level of the active enzyme and its inhibitor. ${ }^{4-7}$ The TIMP family consists of 4 members: TIMP-1, TIMP-2, TIMP-3 and TIMP-4, which act as negative regulators of the degradation processes in the extracellular matrix. The latest evidences suggest that they also participate in cell signalling by regulating cell growth, apoptosis, angiogenesis and genomic instability. $6,8,9$

By analysing TIMP (and TIMP-1 in particular) expression in tumour tissue or circulating TIMP levels in plasma, they were recognized as candidate markers for diagnosis and prognosis in patients with different types of cancer, e.g. colorectal, breast, lung, ovarian, bladder carcinoma ${ }^{6,9}$, but, also in a variety of nonmalignant conditions: asthma, diabetes, cardiovascular and autoimmune diseases. ${ }^{9}$ Increased TIMP-1 plasma levels were found in patients with colorectal cancer compared to healthy controls, and were associated with advanced disease stage and poor prognosis. ${ }^{6,8-12} \mathrm{In}$ metastatic colorectal cancer, elevated level of the inhibitor in plasma was predictive for low probability of response to chemotherapy. ${ }^{13}$ Finally, Unsal et al. reported that matrix metalloproteinase- 9 expression correlates with a poor tumour response to preoperative chemoradiotherapy in patients with locally advanced rectal cancer. ${ }^{14}$

The aim of this study was to determine the predictive and prognostic value of TIMP-1 plasma level for local tumour response to preoperative radiochemotherapy and survival in patients with rectal cancer.

\section{Patients and methods}

\section{Patients and treatment}

Ninety-two patients (63 male, 29 female), aged from 42 to 86 years (mean 73 years), with histologically confirmed non-metastatic rectal cancer of clinical stage I- III, were included in prospective study. They were treated with preoperative radiochemotherapy at the Institute of Oncology in Ljubljana between March 2005 and October 2006. In majority of patients $(76 ; 82.6 \%)$ tumours were staged as cT3
TABLE 1. Characteristics of patients and their tumours ( $N=92)$

\begin{tabular}{|c|c|c|c|c|}
\hline \multicolumn{5}{|l|}{ Patients } \\
\hline \multicolumn{4}{|c|}{ Sex (female/male) } & $29 / 63$ \\
\hline \multicolumn{4}{|l|}{ Age } & $73(42-86)^{*}$ \\
\hline \multicolumn{5}{|c|}{ Primary tumour site } \\
\hline \multicolumn{4}{|c|}{ Lower rectum } & $30(32.6 \%)$ \\
\hline \multicolumn{4}{|c|}{ Middle rectum } & $44(47.8 \%)$ \\
\hline \multicolumn{4}{|c|}{ Upper rectum } & $18(19.6 \%)$ \\
\hline \multicolumn{5}{|c|}{ UICC cTNM-classification } \\
\hline & $\mathrm{N}_{0}$ & $N_{1}$ & $\mathrm{~N}_{2}$ & Total \\
\hline $\mathrm{T}_{1}$ & & & & 0 \\
\hline $\mathrm{T}_{2}$ & 4 & 3 & 0 & 7 \\
\hline $\mathrm{T}_{3}$ & 40 & 33 & 3 & 76 \\
\hline $\mathrm{T}_{4}$ & 0 & 6 & 3 & 9 \\
\hline Total & 44 & 42 & 6 & 92 \\
\hline
\end{tabular}

\begin{tabular}{cl}
\hline Overall stage & \\
\hline Stage I & $4(4.3 \%)$ \\
Stage II & $40(43.5 \%)$ \\
Stage IIIA & $3(3.3 \%)$ \\
Stage IIIB & $42(45.6 \%)$ \\
Stage IIIC & $3(3.3 \%)$ \\
\hline
\end{tabular}

\begin{tabular}{cc}
\hline Histopathological grading & \\
\hline Well differentiated, $G_{1}$ & $14(15.2 \%)$ \\
Moderately differentiated, $G_{2}$ & $59(64.1 \%)$ \\
Poorly differentiated $G_{3}$ & $19(20.7 \%)$ \\
\hline
\end{tabular}

Median (range), in years

UICC = International Union Against Cancer; CTNM = clinical stage tumour-nodes-metastases

and clinically positive regional nodes were found in more than half of the cases $(48 ; 52.2 \%)$ (Table 1$)$. Patients underwent a general clinical examination, including rectal examination by two independent examiners, complete blood tests, chest radiography and abdominopelvic computer tomography (CT), rectoscopy, endoscopic ultrasound (US) and magnetic resonance imaging (MRI) of the pelvis.

Preoperatively, patients were irradiated using CT-based 3-dimensional (3D) planning and four- 
field box technique at a $15 \mathrm{MV}$ linear accelerator. Radiotherapy regimen was comprised of $45 \mathrm{~Gy}$ in 25 fractions to small pelvis with a boost of 5.4 Gy to the primary tumour. In case of cT4 tumour or unresectable disease the booster dose was 9 Gy. Concurrently with irradiation all patients had chemotherapy (ChT), consisted of continuous peroral capecitabine $\left(825 \mathrm{mg} / \mathrm{m}^{2}\right.$ b.i.d., days 1-33, without weekend breaks) or of 2 cycles of intravenous 5-fluorouracil (5-FU) and leucovorine (425 $\mathrm{mg} / \mathrm{m}^{2} /$ day and $20 \mathrm{mg} / \mathrm{m}^{2} /$ day, days 1 to 5 ).

Surgery employing total mesorectal excision (TME) technique was scheduled 6-8 weeks after the completion of chemoradiation and sphincter preservation procedure was done whenever possible. Four cycles of chemotherapy with either capecitabine $\left(1250 \mathrm{mg} / \mathrm{m}^{2}\right.$ bid, days $1-14$, every 3 weeks) or bolus 5-FU and leucovorine $\left(425 \mathrm{mg} / \mathrm{m}^{2} /\right.$ day and $20 \mathrm{mg} / \mathrm{m}^{2} /$ day, days $1-5$, every 4 weeks) were planned postoperatively. The choice of postoperative chemotherapy was left to the oncologist's discretion. After finishing all therapies, patients were followed in 3 month intervals for the first 2 years and thereafter every six months up to the 5 th year.

\section{Determination of plasma TIMP-1 level}

Blood samples were collected in vacutainer EDTA tubes. After centrifugation at $1500 \mathrm{xg}$ at $4^{\circ} \mathrm{C}$ for 10 minutes, plasma was separated and kept at $-80^{\circ} \mathrm{C}$ until analysis. Plasma TIMP-1 concentrations were measured with an enzyme-linked immunosorbent assay (ELISA) using commercially available TIMP-1 ELISA kit, purchased from Oncogen Science, Cambridge (MA), USA. Procedures were performed according to the manufacturer's protocol in duplicate, using diluted samples. All measurements differed by less than $10 \%$ and the mean value was calculated and used for statistical analysis. The inter-assay precision coefficients of variation of TIMP-1 ranged between $3.9 \%$ and $8.8 \%$ at the different levels.

\section{Statistical analysis}

Statistical analysis was performed using personal computer and software statistical package SPSS, version 13 (SPSS Inc., USA). The main endpoints of the study were response to preoperative therapy, locoregional control (LRC, the event was local or regional recurrence), disease-free survival (DFS, the event was local, regional or systemic recurrence), disease-specific survival (DSS, the event was death due to the rectal cancer) and overall survival (OS, the event was death from any cause). MannWhitney test for independent groups was used to assess the differences in plasma TIMP-1 levels and clinicopathological parameters between various groups of patients. The survival of patients was computed from the first day of therapy to the closure date (December 31 ${ }^{\text {st }}$, 2012). The Kaplan-Meier estimate was employed to calculate survival probability $^{15}$, and the differences between plotted curves were tested using the log-rank test. ${ }^{16}$ Independent prognostic value of factors that appeared statistically significant on univariate analysis was tested in multivariate Cox proportional hazard regression analysis. ${ }^{17}$ A two-tailed $\mathrm{P}<0.05$ was considered as statistically significant.

The study protocol was approved by the Medical Ethics Committee at the Ministry of Health of the Republic of Slovenia. All of the included patients gave their informed consent to the voluntary participation in the study.

\section{Results}

\section{Course of treatment and outcome}

Therapy as planned was delivered to 67 (70.8\%) of patients: 16 (17.4\%) patients had no concurrent ChT (history of ischaemic heart disease or other serious comorbidity) and in $9(11.8 \%)$ patients it was omitted before the end of the preoperative treatment due to the acute side effects. Abdominoperineal excision, low anterior resection, and palliative surgery only was performed in 32 (34.8\%), 52 (56.6\%) and $4(4.3 \%)$ patients, respectively. Four (4.3\%) patients had no surgery (refusal 1, disease progression 3). Histopathologically radical resection (R0) was performed in 80 (87\%) patients and pathological complete and partial response rates after preoperative radiochemotherapy were $16.3 \%$ and $34.8 \%$, respectively. Postoperatively, adjuvant ChT was administered to $27(29.3 \%)$ patients and $70.4 \%$ of them received 4 cycles.

Median follow-up time was 68 months (range: 3-93 months) and was 80 months (range: 68-93 months) in survivors. On the close-out date, 42 $(45.7 \%)$ patients were alive, 33 (35.9\%) patients died of rectal cancer whereas in 11 (12\%) patients the causes of death were vascular events (8) and pneumonia, metachronous bronchus cancer and malignant melanoma in 1 patient each, but, in 6 patients the cause of death was unknown. Sites of failure were as follows: local/locoregional 9 (9.8\%), distant 23 (25\%), local/locoregional and distant 5 
TABLE 2. Tissue inhibitor of metalloproteinases (TIMP-1) and clinicopathological variables

\begin{tabular}{|c|c|c|c|}
\hline Clinicopathological variables & $N=92$ & $\begin{array}{l}\text { TIMP-1 level in } \mathrm{ng} / \mathrm{mL} \\
\text { Median (range) }\end{array}$ & $p$-value \\
\hline $\begin{array}{l}\text { Age } \\
<69 \text { years } \\
\geq 69 \text { years }\end{array}$ & $\begin{array}{l}37 \\
55\end{array}$ & $\begin{array}{l}172(55-350) \\
204(22-523)\end{array}$ & NS \\
\hline $\begin{array}{l}\text { Gender } \\
\text { Male } \\
\text { Female }\end{array}$ & $\begin{array}{l}63 \\
29\end{array}$ & $\begin{array}{l}183(22-421) \\
200(79-523)\end{array}$ & NS \\
\hline $\begin{array}{l}\text { cT } \\
\text { сT2+3 } \\
\text { CT4 }\end{array}$ & $\begin{array}{l}83 \\
9\end{array}$ & $\begin{array}{l}187(22-421) \\
227(129-523)\end{array}$ & 0.084 \\
\hline $\begin{array}{l}\mathrm{CN} \\
\mathrm{CNO} \\
\mathrm{CN}+\end{array}$ & $\begin{array}{l}44 \\
48\end{array}$ & $\begin{array}{l}172(66-318) \\
190(22-523)\end{array}$ & NS \\
\hline $\begin{array}{l}\text { Resectability } \\
\text { R0 } \\
\text { R1+R2 }\end{array}$ & $\begin{array}{l}80 \\
8\end{array}$ & $\begin{array}{l}185(22-523) \\
183(82-263)\end{array}$ & NS \\
\hline $\begin{array}{l}\text { pT } \\
\text { рT0+1+2 } \\
\text { pT3+4 }\end{array}$ & $\begin{array}{l}32 \\
56\end{array}$ & $\begin{array}{l}187(22-421) \\
183(66-523)\end{array}$ & NS \\
\hline $\begin{array}{l}\mathrm{pN} \\
\mathrm{pNO} \\
\mathrm{pN}+\end{array}$ & $\begin{array}{l}66 \\
22\end{array}$ & $\begin{array}{l}187(22-362) \\
183(82-523)\end{array}$ & NS \\
\hline $\begin{array}{l}\text { T- downstage } \\
\text { Yes } \\
\text { No }\end{array}$ & $\begin{array}{l}32 \\
56\end{array}$ & $\begin{array}{l}190(22-523) \\
175(66-350)\end{array}$ & NS \\
\hline $\begin{array}{l}\text { N-downstage } \\
\text { Yes } \\
\text { No }\end{array}$ & $\begin{array}{l}72 \\
16\end{array}$ & $\begin{array}{l}190(22-523) \\
168(82-255)\end{array}$ & NS \\
\hline $\begin{array}{l}\mathrm{PCR} \\
\text { Yes } \\
\text { No }\end{array}$ & $\begin{array}{l}15 \\
73\end{array}$ & $\begin{array}{l}182(80-421) \\
187(22-523)\end{array}$ & NS \\
\hline $\begin{array}{l}\text { CEA (mg/L) } \\
<3.4 \\
<3.4\end{array}$ & $\begin{array}{l}44 \\
48\end{array}$ & $\begin{array}{l}187(22-523) \\
185(66-350)\end{array}$ & NS \\
\hline $\begin{array}{l}\text { Platelets count (10E9/L) } \\
<290 \\
<290\end{array}$ & $\begin{array}{l}40 \\
52\end{array}$ & $\begin{array}{l}185(55-421) \\
190(22-523)\end{array}$ & NS \\
\hline $\begin{array}{l}\text { CRP }(\mathrm{mg} / \mathrm{L}) \\
<12 \\
\geq 12\end{array}$ & $\begin{array}{l}73 \\
19\end{array}$ & $\begin{array}{l}175(22-421) \\
227(92-523)\end{array}$ & 0.031 \\
\hline $\begin{array}{l}\text { Recurrence } \\
\text { Yes } \\
\text { No }\end{array}$ & $\begin{array}{l}37 \\
55\end{array}$ & $\begin{array}{l}187(66-523) \\
190(22-421)\end{array}$ & NS \\
\hline $\begin{array}{l}\text { Death from rectal cancer } \\
\text { Yes } \\
\text { No }\end{array}$ & $\begin{array}{l}33 \\
59\end{array}$ & $\begin{array}{l}200(66-523) \\
172(22-421)\end{array}$ & NS \\
\hline $\begin{array}{l}\text { Dead } \\
\text { Yes } \\
\text { No }\end{array}$ & $\begin{array}{l}50 \\
42 \\
\end{array}$ & $\begin{array}{l}207(55-523) \\
167(22-362)\end{array}$ & 0.02 \\
\hline
\end{tabular}

$\mathrm{NS}=$ not significant; $\mathrm{PCR}=$ pathologic complete remission; $\mathrm{CEA}=$ carcinoembriogenic antigene, $\mathrm{CRP}=\mathrm{C}$ reactive protein

(5.5\%). The 5-year LRC, DFS, DSS and OS rates for all patients were $80.2 \%, 56.4 \%, 63.7 \%$ and $52.2 \%$, respectively.

\section{TIMP-1 and prognosis}

The median TIMP-1 level was $185 \mathrm{ng} / \mathrm{mL}$ (range: $22-523 \mathrm{ng} / \mathrm{mL}$ ) and the mean level ( \pm standard deviation) was $192( \pm 87) \mathrm{ng} / \mathrm{mL}$. Serum TIMP-1 levels were found to be significantly increased in patients with preoperative $\mathrm{CRP}>12 \mathrm{mg} / \mathrm{L}$ and in those who died from rectal cancer and were marginally increased in patients who had cT4 tumours. No correlations were established for age, gender, $\mathrm{cN}$ stage, CEA level, platelets count, histopathological grade, response to preoperative therapy, resectability and disease re-appearance.

On univariate analysis, various parameters favourablyinfluenced oneormoresurvivalendpoints: TIMP-1 < 170 ng/mL (Figure 1), CRP < 12 mg/L, platelets count $<29010^{9} \mathrm{~L}, \mathrm{CEA}<3.4 \mathrm{mg} / \mathrm{L}$, age $<69$ years, male gender, early stage disease $(\mathrm{cN} 0$ 


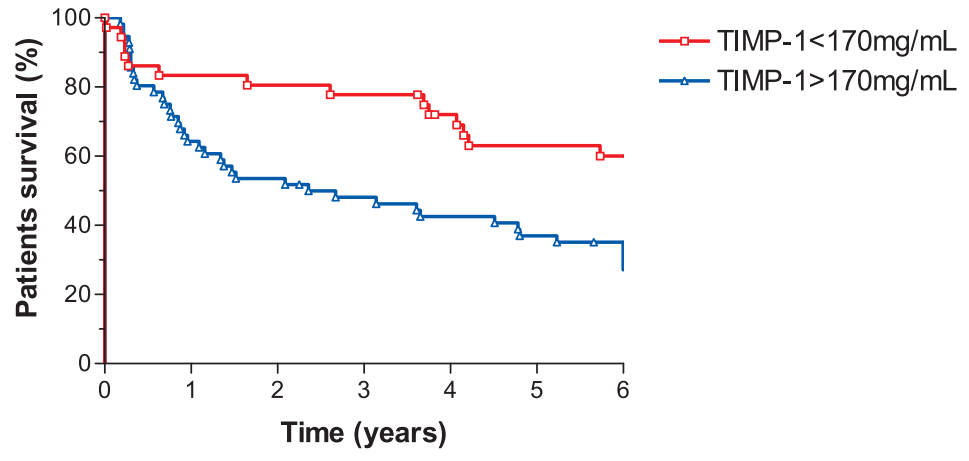

FIGURE 1. Overall survival and tissue inhibitor of metalloproteinases 1 (TIMP-1).

and/or cT2-3), radical surgery (R0) and response to preoperative radiochemotherapy (Table 2). The following parameters were introduced in multivariate model to test their independent prognostic value: CRP, T-downstage, N-downstage and pretreatment TIMP-1 level. LRC was favourably influenced by $\mathrm{N}$-downstage (Hazard ratio $[\mathrm{HR}]=6.10 ; 95 \%$ confidence interval $[\mathrm{CI}]: 1.78-20.89$; $p=0.004) ;$ DFS by CRP $(\mathrm{HR}=3.09 ; 95 \%$ CI: $1.33-7.18 ; p=0.009)$ and $\mathrm{N}$-downstage (HR $=3.66 ; 95 \%$ CI: $1.58-8.52 ; p=0.003)$; DSS by CRP $(\mathrm{HR}=2.77 ; 95 \% \mathrm{CI}: 1.13-6.76 ; p=0.03)$ and $\mathrm{N}$-downstage $(\mathrm{HR}=3.88 ; 95 \% \mathrm{CI}: 1.62-9.32 ; p=0.002)$; OS by TIMP-1 level (HR = 2.15; 95\% CI: 1.01-4.56; $p=0.047), \mathrm{CRP}(\mathrm{HR}=2.14 ; 95 \% \mathrm{CI}: 1.08-4.25 ; p=0.029)$ and $\mathrm{N}$-downstage $(\mathrm{HR}=2.89$; 95\% CI: 1.35-6.18; $p=0.006$ ) (Table 3).

\section{Discussion}

Several authors reported on the positive correlation between elevated serum or tissue TIMP-1 levels and increased aggressiveness of the disease. We can assume that elevated TIMP-1 levels reflect the degree of proteolytic activity which is an essential process implicated in invasiveness of tumour cells. Therefore, it was hypothesized that if TIMP-1 is predictive for relapse and survival $6,8,10,11,18,19$ it could be used to distinguish between patients with higher and those with lower risk for the disease recurrence. Detailed knowledge on risk level for disease re-appearance would allow us to avoid over- or undertreatment. Furthermore, the ability to predict efficiency of specific type of therapy, e.g. preoperative radiochemotherapy in rectal cancer patients, could help us to tailor the entire "treatment package" more according to individual tumour characteristics which are usually not taken into consideration.

In this view, our intention was to assess the predictive value of serum TIMP-1 levels in cohort of patients with rectal cancer who were treated with preoperative radiochemotherapy. The studied population was representative in regards to the treatment results such as percentage of radical resections $(87 \%)$, T- and N-downstaging (34.8\%), pathological complete responses $(16.3 \%)$ and survivals (at 5 years: LRC-80.2\%, DFS-56.4\%, DSS-63.7\%, and OS-52.2\%) which are from all aspects comparable with the results of other researchers. ${ }^{20,21}$

When analysing the association between established clinicopathological parameters and TIMP-1, we found elevated TIMP-1 levels in patients with higher cT-stage and those who died from rectal cancer or had increased CRP before the start of the preoperative treatment. In several studies, higher TIMP-1 levels were associated with advanced stage of the disease and poor prognosis.6,10,11,22 On the other hand, Holten-Anderson et al. did not find any differences in the TIMP-1 levels between Dukes stage A, B or C; patients with Dukes D rectal can-

TABLE 3. Multivariate analysis of survival

\begin{tabular}{|c|c|c|c|c|c|c|c|c|c|c|c|c|}
\hline \multirow[t]{2}{*}{ Parameter } & \multicolumn{3}{|c|}{ Locoregional control } & \multicolumn{3}{|c|}{ Disease-free survival } & \multicolumn{3}{|c|}{ Disease-specific survival } & \multicolumn{3}{|c|}{ Overall survival } \\
\hline & HR & $95 \% \mathrm{Cl}$ & P-VALUE & HR & $95 \% \mathrm{Cl}$ & P-VALUE & HR & $95 \% \mathrm{Cl}$ & P-VALUE & HR & $95 \% \mathrm{Cl}$ & P-VALUE \\
\hline $\begin{array}{l}\text { TIMP-1 } \\
<170 \mathrm{ng} / \mathrm{mL} / \\
\geq 170 \mathrm{ng} / \mathrm{mL}\end{array}$ & 1.33 & $0.33-5.31$ & NS & 1.16 & $0.50-2.72$ & NS & 1.34 & $0.53-3.37$ & NS & 2.15 & $1.01-4.56$ & 0.047 \\
\hline $\begin{array}{l}\text { T- downstage } \\
\text { Yes/No }\end{array}$ & 2.76 & $0.59-12.99$ & NS & 1.93 & $0.82-4.57$ & NS & 1.72 & $0.71-4.17$ & NS & 1.55 & $0.79-3.01$ & NS \\
\hline $\begin{array}{l}\text { N- downstage } \\
\text { Yes/No }\end{array}$ & 6.10 & $1.78-20.89$ & 0.004 & 3.66 & $1.58-8.52$ & 0.003 & 3.88 & $1.62-9.32$ & 0.002 & 2.89 & $1.35-6.18$ & 0.006 \\
\hline $\begin{array}{l}\text { CRP } \\
<12 \mathrm{mg} / \mathrm{L} / \\
\geq 12 \mathrm{mg} / \mathrm{L}\end{array}$ & 2.75 & $0.68-11.17$ & NS & 3.09 & $1.33-7.18$ & 0.009 & 2.77 & $1.13-6.76$ & 0.03 & 2.14 & $1.08-4.25$ & 0.029 \\
\hline
\end{tabular}

$\mathrm{HR}=$ hazard ratio; $\mathrm{Cl}$-confidence interval; NS = not significant 
cer, however, had significantly increased TIMP-1 level compared to less advanced stages. ${ }^{23}$ In many studies TIMP-1 was reported to be in positive correlation with patients' age $\mathrm{e}^{6,10,22,24}$, although Tayebjee et al. and our study found just the opposite. ${ }^{25}$ The relationship between TIMP-1 and CRP can be explained with the fact that they both participate in the processes of inflammation. Frederiksen et al. who systematically followed TIMP-1 levels before and after the surgery suggested that prolonged recovery due to postoperative infections may contribute to prolonged increase of plasma TIMP-1 level. ${ }^{9}$

In the present study, no correlation between TIMP-1 levels and the response to preoperative radiochemotherapy was observed, although Sørensen et al. reported that TIMP-1 can be predictive for the response to chemotherapy in colon carcinoma ${ }^{13}$ and Unsal et al. found that positive MMP9 expression correlated with poor tumour response in patients with locally advanced rectal cancer undergoing preoperative radiochemotherapy. ${ }^{14}$

We also analysed the relationship between TIMP-1 levels and CEA, which is well recognized and still the only recommended marker used in the diagnosis and follow-up of patients with colorectal cancer. As confirmed in several studies, increased pretreatment CEA levels reflect an advanced disease stage and are forecasting an increased risk of recurrence and poor prognosis. ${ }^{26}$ However, similar as Holten-Andersen et al. ${ }^{23}$ we also did not find any association between TIMP-1 and CEA levels.

In Cox multivariate analysis we confirmed that patients in whom $\mathrm{N}$-downstaging resulted from preoperative radiochemotherapy had more favourable LRC, DFS, DSS and OS, but patients with T-downstage did not have better survival at all. Furthermore, in patients younger than 69 years we did not find better survival rates, which is opposite to the results of other studies. ${ }^{27,28}$ Finally, we found increased TIMP-1 levels $(\geq 170 \mathrm{mg} / \mathrm{mL})$ to be an independent risk factor for worse OS ( $p=0.047)$. This observations are consistent with the reports of other authors who also identified higher TIMP-1 levels as being a negative predictor of shorter survival. 6,8-13,18,19,23 To the opposite, Ishida et al. were not able to confirm any prognostic value of TIMP-1 for colorectal cancer patients. ${ }^{24}$

\section{Conclusions}

Although we did not find any association between pretreatment serum TIMP-1 levels and primary tu- mour response to preoperative radiochemotherapy in our cohort of patients with rectal cancer, TIMP-1 levels were recognized as an independent prognostic factor for OS in these patients.

\section{References}

1. Kim DY, Jung KH, Kim TH, Kim DW, Chang HJ, Jeong JY, et al. Comparison of 5 -fluorouracil/leucovorin and capecitabine in preoperative chemoradiotherapy for locally advanced rectal cancer. Int J Radiat Oncol Biol Phys 2007; 67: 378-84.

2. Chan AK, Wong AO, Jenken DA. Preoperative capecitabine and pelvic radiation in locally advanced rectal cancer-is it equivalent to 5 -FU infusion plus leucovorin and radiotherapy? Int J Radiat Oncol Biol Phys 2010; 76: 1413-9.

3. Velenik V, Ocvirk J, Oblak I, Anderluh F. Cetuximab in preoperative treatment of rectal cancer - term outcome of the XERT trial. Radiol Oncol 2012; 46: 252-7.

4. Pesta M, Topolcan O, Holubec L Jr, Rupert K, Cerna M, Holubec LS, et al. Clinicopathological assesment and quantitative estimation of the matrix metalloproteinases MMP-2 and MMP-7 and the inhibitors TIMP- 1 and TIMP- 2 in colorectal carcinoma tissue samples. Anticancer Res 2007; 27: 1863-7.

5. Li M, Yamamoto $H$, Adachi $Y$, Maruyama $Y$, Shinomura Y. Role of matrix metaloproteinase-7 (matrilysin) in human cancer invasion, apoptosis, growth and angiogenesis. Exp Biol Med 2006; 231: 20-7.

6. Giaginis C, Nikiteas N, Margeli A, Tzanakis N, Rallis G, Kouraklis G, et al. Serum tissue inhibitor of metalloproteinase 1 and 2 (TIMP-1 and TIMP-2) levels in colorectal cancer patients: association with clinicopathological variables and patients survival. Int J Biol Markers 2009; 24: 245-52.

7. Alo-Aho R, Kahari VM: Colagenases in cancer. Biochemie 2005; 87: 273-86.

8. Sørensen NM, Sørensen IV, Wúrtz S $\varnothing$, Schrohl AS, Dowell B, Davis G, et al. Biology and potential clinical implications of tissue inhibitor of metalloproteinases-1 in colorectal cancer treatment. Scan J Gastroenterol 2008; 43: $774-86$.

9. Frederiksen CB, Lomholt AF, Lottenburger T, Davis GJ, Dowell BL, Blankenstein MA, et al. Assesment of the biological variation of plasma tissue inhibitor of metalloproteinase-1. Int J of Biological Markers 2008; 23: 42-7.

10. Wass ET, Hendriks T, Lomme RM, Woblles T. Plasma levels of matrix metalloproteinase-2 and tissue inhibitor of metalloproteinase-1 correlate with disease stage and survival in colorectal cancer patients. Dis Colon Rectum 2005; 48: 700-10.

11. Kahlert C, Bandapalli OR, Schirmacher P, Weitz J, Brand K. Invasion frontspecific overexpression of tissue inhibitor of metalloproteinase-1 in liver metastases from colorectal cancer. Anticancer Res 2008; 28: 1459-66.

12. Oblak I, Anderluh F, Velenik V, Mozina B, Ocvirk J, Ciric E, et al. Differences in plasma TIMP-1 levels between healthy people and patients with rectal cancer stage II-III. Radiol Oncol 2011; 45: 209-12.

13. Sørensen NM, Byström P, Christensen IJ, Berglund A, Nielsen HJ, Brüner N et al. TIMP-1 is significantly associated with objective response and survival in metastatic colorectal cancer patients reciving combination of irinotecan, 5-fluorouracil, and folinic acid. Clin Cancer Res 2007; 13: 4117-22.

14. Unsal D, Under A, Akyurek N, Erpolat P, Dursun A, Pak Y. Matrix metalloproteinase-9 expression correlates with tumor response in patients with locally advanced rectal cancer undergoing preoperative chemoradiotherapy. Int $J$ Radiol Oncol Biol Phys 2007; 67: 196-203.

15. Kaplan EL, Meier P. Nonparametric estimation from incomplete observations. J Am Stat Assoc 1958; 53: 457-81.

16. Peto R, Pike MC, Armitage P, Breslow NE, Cox DR, Howard SV, et al. Design and analysis of randomized clinical trials requiring prolonged observation of each patient. II. Analysis and examples. Br J Cancer 1977; 35: 1-39.

17. Cox DR. Regression models and life-tables. J R Stat Soc Bull 1972; 34: 187 220.

18. Holten-Andersen $M N$, Nielsen $H J$, Sørensen $S$, Jensen V, Brüner $N$, Christensen IJ. Tissue inhibitor of metalloproteinases-1 in the postoperative monitoring of colorectal cancer. Eur J Cancer 2006; 42: 1886-96. 
19. Yakawa N, Yoshikawa T, Akaike $M$, Sugimasa $Y$, Rino $Y$, Masuda $M$, et al. Impact of plasma tissue inhibitor of matrix metalloproteinase-1 on longterm survival in patients with colorectal cancer. Oncology 2007; 72: 205-8.

20. Velenik V, Oblak I, Anderluh F. Long-term results from a randomized phase II trial of neoadjuvant combined-modality therapy for locally advanced rectal cancer. Radiat Oncol 2010; 5: 88-96.

21. Dunst J, Debus J, Rudat V, Wulf J, Budach W, Hoelscher T, et al. Neoadjuvant capecitabine combined with standard radiotherapy in patients with locally advanced rectal cancer: mature results of a phase II trial. Strachlenther Oncol 2008; 184: 450-6.

22. Holten-Anderson MN, Christensen IJ, Nielsen HJ, Lilja H, Murphy G, Jensen $\mathrm{V}$, et al. Measurement of the non-complex free fraction of tissue inhibitor of metalloproteinases I in plasma by immunoassay. Clin Chem 2002; 48 : 1305-13.

23. Holten-Anderson $\mathrm{M}$, Christensen IJ, Nilberg M, Bendahl PO, Nielsen $\mathrm{HJ}$ Brúnner $\mathrm{N}$, et al. Association between preoperative plasma levels of tissue inhibitor of metalloproteinases 1 and rectal cancer patient survival: a validation study. Eur J of Cancer 2004; 40: 64-72.

24. Ishida H, Murata N, Hayashi Y, Tada M, Hashimoto D. Serum levels of tissue inhibitor of metalloproteinases-1 (TIMP-1) in colorectal cancer patients. Surg Today 2003; 33: 885-92.

25. Tayebjee MH, Lip GYH, Blann AD, MacFadyen RJ. Effects of age, gender, ethnicity, diurnal variations and exercise on circulating levels of matrix metalloproteinases (MMP)-2 and-9, and their inhibitors, tissue inhibitors of matrix metalloproteinases (TIMP)-1 and -2. Thromb Res 2005; 115: 205-10.

26. Duffy MJ, van Dalen A, Haglund C, Hansson L, Klapdor R, Lamerz R, et al. Clinical utility of biochemical markers in colorectal cancer: European Group on Tumour Markers (EGTM) guidelines. Eur J Cancer 2003; 39: 718-27.

27. Sprenger T, Rothe H, Jung K, Christiansen H, Conradi LC, Ghadimi BM, et al. Stage II/III rectal cancer with intermediate response to preoperative radiochemotherapy: do we have indications for individual risk stratification? World J Surg Oncol 2010; 8: 27.

28. Laohavinij S, Maneechavakaiorn J, Techatanol P. Prognostic factors for survival in colorectal cancer patients. J Med Assoc Thai 2010; 93: 1156-66. 


\title{
Comparison of continuous local anaesthetic and systemic pain treatment after axillary lymphadenectomy in breast carcinoma patients - a prospective randomized study
}

\author{
Branka Strazisar ${ }^{1}$, Nikola Besic ${ }^{2}$ \\ ${ }^{1}$ Department of Anaesthesiology and ${ }^{2}$ Department of Surgical Oncology, Institute of Oncology, Ljubljana, Ljubljana, Slovenia
}

Radiol Oncol 2013; 47(2): 145-153.

Received 8 December 2012

Accepted 22 December 2012

Correspondence to: Nikola Besic, MD, PhD, Department of Surgical Oncology, Institute of Oncology, Zaloska 2, 1000 Ljubljana, Slovenia. Phone: +386 15879 953; Fax: +386 15879 400; E-Mail: nbesic@onko-i.si

Disclosure: No potential conflicts of interest were disclosed.

\begin{abstract}
Background. Acute pain after axillary lymphadenectomy is often related mainly to axillary surgery. The aim of the prospective randomized study was to find out if continuous wound infusion of local anaesthetic reduces postoperative pain, consumption of opioids and the incidence of chronic pain compared to the standard intravenous piritramide analgesia after axillary lymphadenectomy in breast carcinoma patients.

Methods. Altogether 60 patients were enrolled in the prospective randomized study; half in wound infusion of local anaesthetic and half in the standard (piritramide) group.

Results. In the recovery room and on the first day after surgical procedure, the wound infusion of local anaesthetic group reported less acute and chronic pain, a lower consumption of piritramide and metoclopramide, but their alertness after the surgical procedure was higher compared to the standard group.

Conclusions. After axillary lymphadenectomy in breast carcinoma patients, wound infusion of local anaesthetic reduces acute pain and enables reduced opioid consumption, resulting in less postoperative sedation and a reduced need for antiemetic drugs. After wound infusion of local anaesthetic there is a statistical trend for reduction of chronic pain.
\end{abstract}

Key words: breast carcinoma; pain treatment; wound infusion of local anaesthetic; elastomeric pump

\section{Introduction}

The sense of pain is an individual experience; it depends on pain memories and present circumstances. ${ }^{1}$ Breast cancer surgery can be painful, but acute pain is often related mainly to axillary surgery. Furthermore, breast surgery can be emotionally distressing. ${ }^{2}$ The risk of clinically meaningful acute pain was increased in patients with preoperative anxiety. ${ }^{3}$ Surgery and inflammation that follow the intervention, activate peripheral nociceptors in the skin, ligaments and muscles. A noxious stimulus is propagated by thin, unmyelinated $C$ type fibres and thinly myelinated A-delta fibres to the central nervous system. ${ }^{4}$ During axillary lymph node dissection, the surgeon should avoid an injury of the long thoracic and thoracodorsal nerve, because the lesion of these nerves causes a muscular deficit and results in physical deformities. ${ }^{5}$ However, the intercostobrachial nerves are often injured or cut during axillary surgery. Already a small lesion of these nerves can cause pain. ${ }^{6}$ Despite a meticulous surgical technique; there is a $35 \%-50 \%$ risk of developing numbness, pain, or paraesthesia.

Patients who develop pain or paraesthesia are at risk of progression to chronic pain syndromes. Five years after axillary dissection, as many as 30\% of patients continue to experience symptoms. ${ }^{7,8}$ 
Chronic pain syndrome after axillary dissection for breast carcinoma is known as post-mastectomy syndrome. ${ }^{9}$ The chronic pain syndrome is often a result of inadequately treated acute pain. ${ }^{10}$ Persistent pain can be avoided through continuous use of a sufficient level of pain medication. ${ }^{9}$ Perioperative analgesia has traditionally been provided by opioid analgesics. Large doses of opioids can be associated with an increased incidence of postoperative complications: respiratory depression, sedation, nausea and vomiting, pruritus and ileus. ${ }^{11,12}$ Therefore, anaesthesiologists and surgeons are increasingly turning to non-opioid analgesic techniques for managing pain during the perioperative period in order to minimize the adverse effects of analgesic medications. ${ }^{12}$ Local anaesthetics can improve postoperative pain management. ${ }^{11}$ They suppress the afferent nociceptive signal and inflammatory reaction. ${ }^{13}$ Local anaesthetics for postoperative analgesia are used in many fields of surgery: orthopaedics, abdominal surgery, gynaecology, urology, cardiothoracic surgery and breast cancer surgery. ${ }^{14}$ Continuous administration of local anaesthetics into the wound via a catheter placed directly at the end of surgery represents a simple and efficacious means to provide postoperative analgesia. ${ }^{15,16}$ At the Institute of Oncology in Ljubljana, intravenous administration of the opioid piritramide is a standard treatment of perioperative pain. Piritramide is used because it has lower frequency of side effects, i.e. vomiting, respiratory depression or cardiovascular instability, compared to morphine. ${ }^{17}$ Based on good results of our retrospective data; we wanted to prove the superiority of the continuous local anaesthetic infusion over the standard intravenous analgesia after axillary dissection in breast cancer patients. We hypothesized that a continuous infusion of the local anaesthetic through a wound catheter after axillary dissection in breast carcinoma patients reduces acute pain more effectively than the standard intravenous opioid analgesia. Our prospective randomized study was also aimed at testing if the patients with a continuous infusion of the local anaesthetic have a lower consumption of opioids, a lower need for antiemetic drugs, lower sedation, and less frequent chronic pain compared to the standard opioid based analgesia group of patients.

\section{Patients and methods}

\section{Methods}

Patients listed for axillary dissection because of breast cancer were screened preoperatively dur- ing the routine anaesthetic assessment. Allergies to local anaesthesia, male gender or dependence on analgesics were the criteria excluding patients from our study. Female patients undergoing breast cancer surgery with axillary dissection were randomized into a group having a wound catheter with elastomeric pump, or into the standard piritramide treatment group. From December 2010 to July 2011, a total of 60 patients were enrolled: 30 patients in the test group and 30 in the standard group.

The study was reviewed by the appropriate Ethics Committee and performed in accordance with the ethical standards laid down in an appropriate version of the 1964 Declaration of Helsinki. Our study was approved by the Institutional Review Board and conducted with the understanding and consent of the involved human subjects.

The surgical procedure was performed during general anaesthesia. Anaesthetic technique for all the patients was the same. All of them received a single prophylactic dose of antibiotic before the beginning of the surgical procedure. Patients without diabetes received $4 \mathrm{mg}$ of dexamethasone. All patients were given $1 \mathrm{mg}$ of granisetron in order to prevent nausea. Before the end of the surgical procedure, all patients received our standard analgesic infusion mixture, which contained $7.5 \mathrm{mg}$ of piritramide, $2.5 \mathrm{~g}$ metamizole and $10 \mathrm{~g}$ of metoclopramide.

\section{Test group of patients}

Before wound closure, 30 patients, who were randomized into the test group, received a fenestrated wound catheter, which was placed into their wound cavity near to the axillary vein and upon the whole length over the upper side of the wound. The wound catheter was fenestrated in the distal part, $15 \mathrm{~cm}$ in length. A bolus of $15 \mathrm{ml}$ of $0.25 \%$ levobupivacaine was injected in the wound through the catheter immediately after wound closure. Soon after the administration of bolus, the elastomeric pump was connected to the wound catheter.

Surgical drains and the fenestrated catheter were clamped for five minutes after the administration of levo-bupivacaine in order to enable the absorption of levo-bupivacaine from the bolus. After five minutes, clamps on surgical drains were released in order to evacuate seroma. After another 15 minutes, the clamp on fenestrated catheter was also released, enabling a continuous infusion of levo-bupivacaine. The amount of the local anaesthetic in the elastomeric pump was $100 \mathrm{~mL}$ at the 
TABLE 1. Characteristics of patients

\begin{tabular}{|c|c|c|c|c|}
\hline Number of patients & & 30 & 30 & - \\
\hline Age (years) - mean & & 57.4 & 62.9 & 0.79 \\
\hline Height $(m)$ - mean & & 1.62 & 1.637 & 0.43 \\
\hline Weight (kg) - mean & & 72.7 & 73.8 & 0.76 \\
\hline Associated diseases & & 19 & 24 & 0.25 \\
\hline Diabetes mellitus & & 4 & 8 & 0.33 \\
\hline Fibromyalgia & & 0 & 1 & - \\
\hline Type of invasive carcinoma & $\begin{array}{l}\text { Ductal } \\
\text { Lobular }\end{array}$ & $\begin{array}{c}28 \\
2\end{array}$ & $\begin{array}{c}27 \\
3\end{array}$ & 1.00 \\
\hline Gradus & $\begin{array}{l}\text { I } \\
\text { II } \\
\text { III }\end{array}$ & $\begin{array}{c}0 \\
11 \\
19\end{array}$ & $\begin{array}{c}1 \\
11 \\
18\end{array}$ & 1.00 \\
\hline Metastatic lymph nodes - mean & & 5.2 & 5.3 & 0.37 \\
\hline Resected lymph nodes - mean & & 17.3 & 19.3 & 0.15 \\
\hline Hormone receptors positive & & 24 & 26 & 0.73 \\
\hline HER-2 positive & & 5 & 6 & 1.00 \\
\hline
\end{tabular}

flow rate of $2 \mathrm{~mL}$ per hour. The catheter was removed 50 hours later, when the elastomeric pump reservoir was empty.

\section{Control group of patients}

The control patients, i.e. those who received the standard intravenous piritramide treatment, were on a continuous intravenous infusion with piritramide $(30 \mathrm{mg})$, metoclopramide $(20 \mathrm{mg})$ and metamizole $(2.5 \mathrm{~g})$ in total amount of $100 \mathrm{~mL}$ of saline until the next morning. The rate of infusion was between $3 \mathrm{~mL} / \mathrm{h}$ and $6 \mathrm{~mL} / \mathrm{h}$. The nursing staffs were instructed to maintain the lowest rate of drip infusion, which relieved the patient of her pain. The volume of infusion has been registered.

Both groups of patients could get the intravenous bolus of a rescue analgesic or an antiemetic drug whenever needed. In a case of severe pain, the patient received a bolus of $3 \mathrm{mg}$ of piritramide. A total consumption of all intravenous drugs used during the first 24 hours after the surgical procedure was registered.

\section{Pain measurement}

Pain was measured using a standard visual analogue scale (VAS) score in ranging from 0 to 10 . The first measurement was made in the recovery room. Thereafter, pain was measured three, six and nine hours after the surgical procedure. Over the next two days, pain was measured every eight hours. Six hours after the surgical procedure, we measured their alertness using the Observer's Assessment of Alertness/Sedation Scale (OAA/S Scale) in a composite way. ${ }^{18}$ The OAA/S Scale score is composed of four categories: 1) responsiveness, 2) speech, 3) facial expression, and 4) eyes. The composite score ranges from 1 (deep sleep) to 5 (alert). The result is the lowest level observed by the nurse in any one of the four component scores.

In case of nausea or vomiting, the patient received a bolus of an antiemetic drug, the first one being metoclopramide. If no relief was achieved, $1 \mathrm{mg}$ of granisetron or, with persistent nausea, $1.25 \mathrm{mg}$ of droperidol was administered intravenously. On the first postoperative day, all patients 
TABLE 2. Treatment of patients and chronic pain after adjuvant therapy

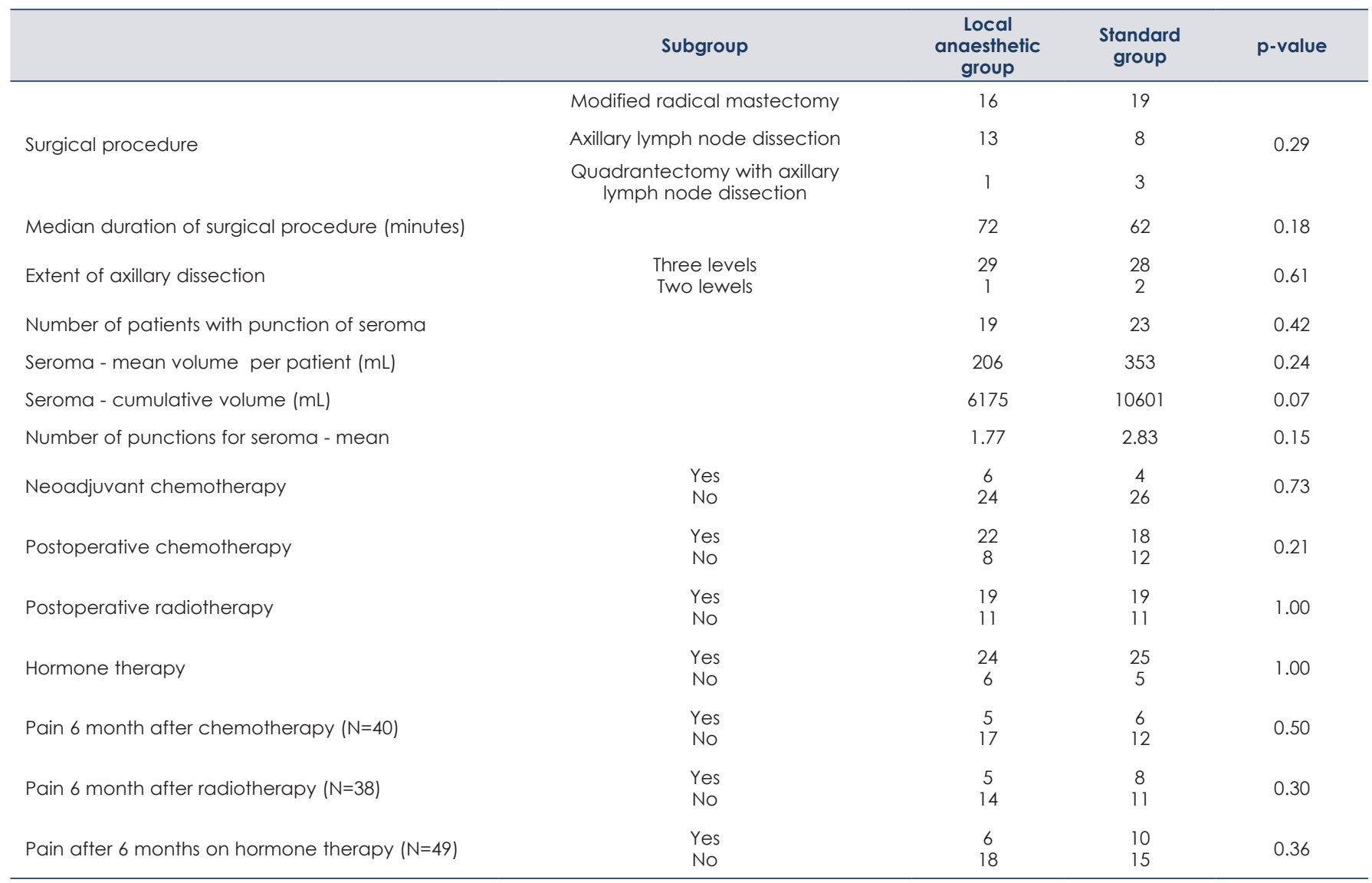

received analgesics in the form of tablets. They were administered $100 \mathrm{mg}$ of diclofenac, a combination of paracetamol and tramadol and, in case of nausea, an antiemetic drug. The consumption of drugs during hospitalization was registered.

After surgical procedure the majority of patients had adjuvant treatment: chemotherapy $(67 \%)$, irradiation (63\%) and/or hormone therapy (82\%).

All complications (inflammation, hematoma and others) were recorded. Three months after the surgical procedure the patients were asked about pain in the postoperative area or the upper extremities. All patients were examined and asked about neuropathic pain in the axilla, shoulder, arm or chest wall six months after the surgical procedure.

\section{Statistical analysis}

The student t-test or the Mann-Whitney $U$ test was conducted according to the data distribution. The association between categorical variables was tested by chi $^{2}$ or Fisher's exact test, as appropriate. All comparisons were two-sided and a p-value of $\leq 0.05$ was considered statistically significant. Statistical packages PASW 18 (SPSS Inc., Chicago, IL, USA) and R 2.11.1 (R Foundation for Statistical Computing, Vienna, Austria) were used for the analysis.

\section{Results}

The mean age of patients was 60 years (range 3084), height $163 \mathrm{~cm}$ (range 150-176), weight $73 \mathrm{~kg}$ (range 43-114), and body mass index (BMI) 27.4 (range 15.4-41.4). There were no significant differences between both study groups either in BMI, American Society of Anaesthesiology (ASA) score, comorbidities (Table 1), length of surgical procedure, extent of lymph node dissection, volume of seroma or number of punctions of seroma (Table 2).

All lymphadenectomies were performed by eleven experienced surgeons. There were no significant differences between various surgeons either in VAS score during hospital stay $(\mathrm{p}=0.66)$ or in rate of chronic pain after six months $(\mathrm{p}=0.32)$. 

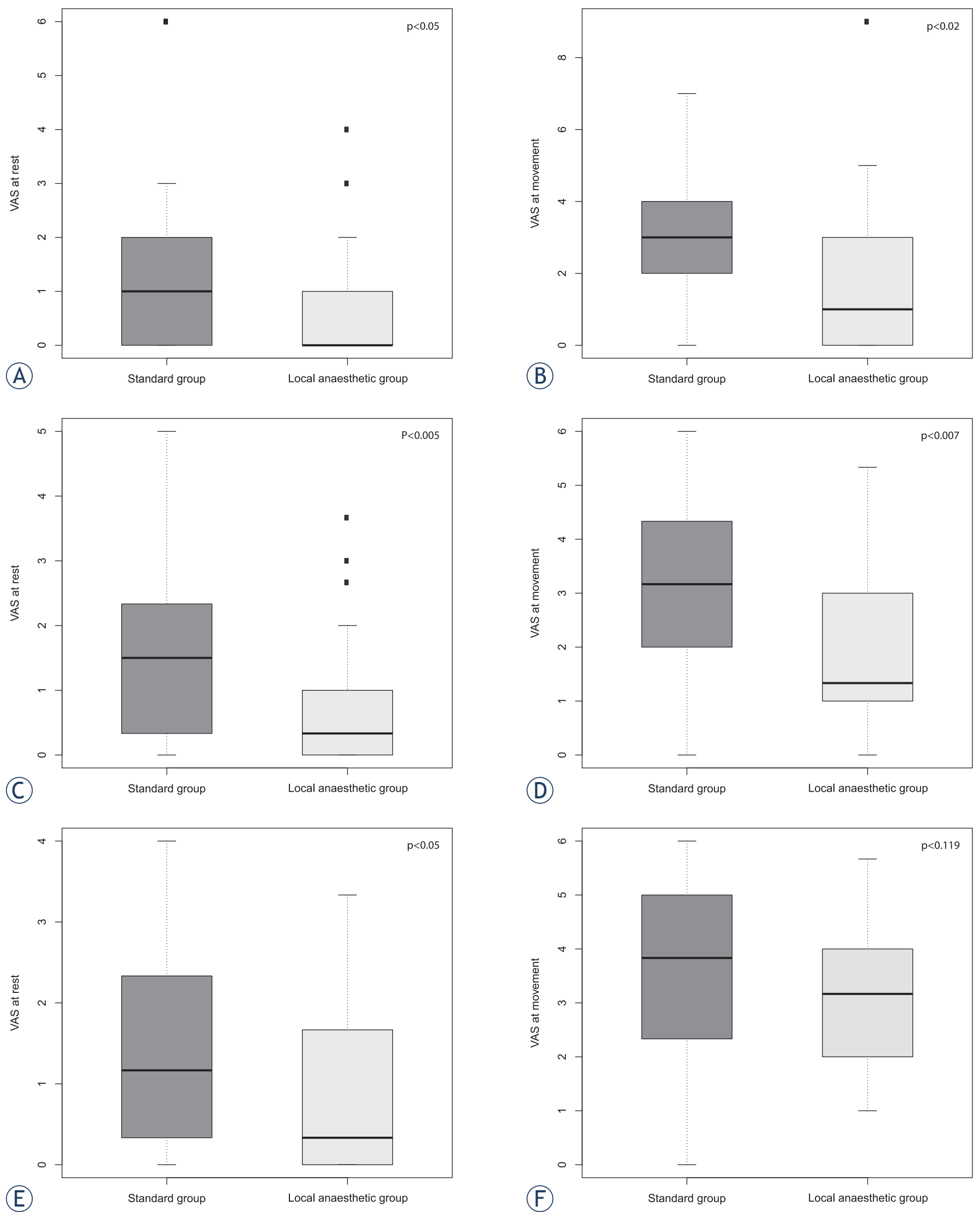

FIGURE 1. Visual analogue scale (VAS) scores at rest and at movement in the recovery room (A, B) on the day of the surgery (C, D) on the first postoperative day (E, F). 
TABLE 3. Pain, consumption of drugs and alertness in local anaesthetic group and standard group of patients

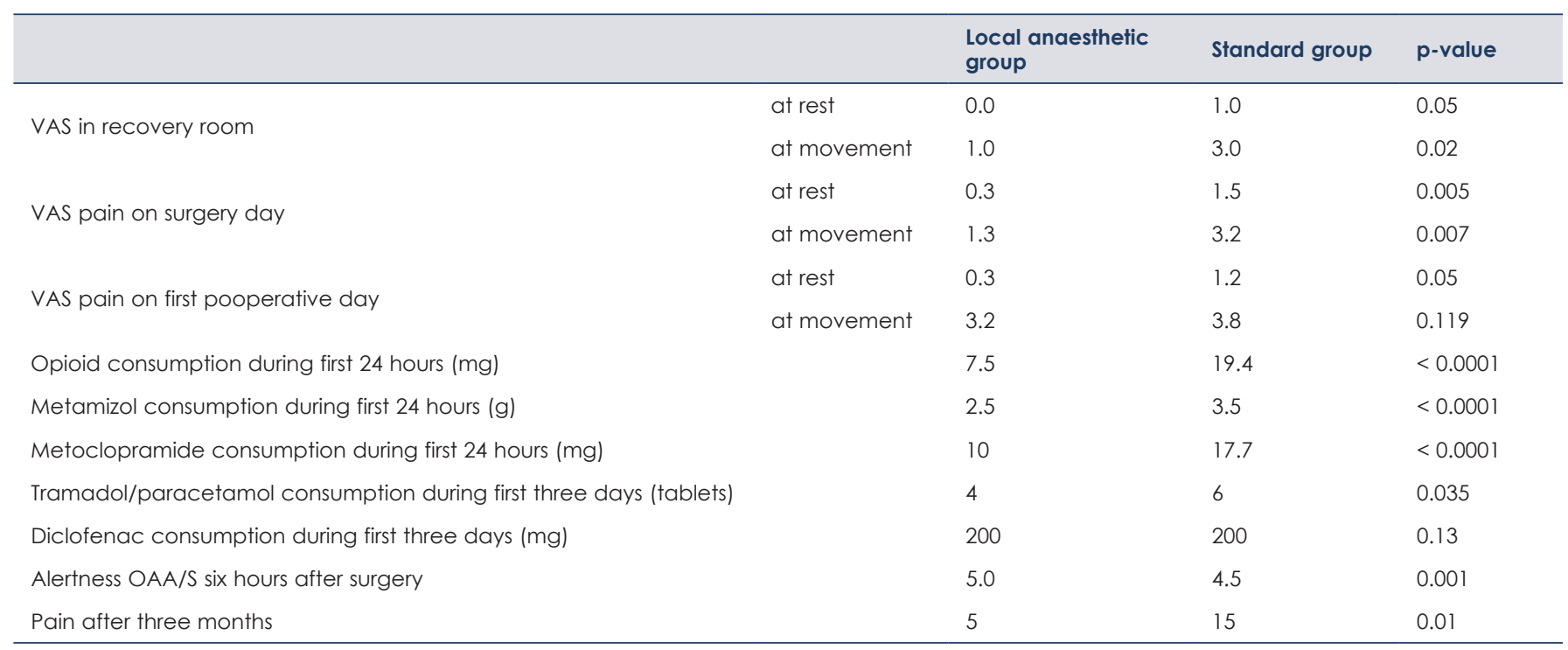

Data is presented as the median value or number.

$\mathrm{VAS}=$ visual analogue scale; OAA/S = Observer's Assessment of Alertness/Sedation

\section{Acute pain}

Figure 1 shows VAS scores at rest and during movement in the recovery room on the day of the surgery and on the first postoperative day. Data about postoperative pain in VAS scores are presented in Table 3.

\section{Opioid consumption}

Consumption of piritramide during the first 24 hours after the surgical procedure was smaller in the test group compared to the control group $(\mathrm{p}<$ 0.0001) (Figure 2). Alertness, as measured six hours after the surgical procedure, was higher in the test group compared to the test group $(p=0.001)$.

\section{Nausea}

Patients in the test group reported less nausea than patients in the standard group. Consumption of metoclopramide during the first 24 hours (Figure 3 ) after the surgical procedure was also smaller in the test group compared to the standard group ( $\mathrm{p}<$ 0.0001).

\section{Complications}

No local signs of infection were observed in the area, where the wound catheter was inserted. All microbiological samples taken were negative. There were no significant differences in the complications following the surgical procedure between the two groups. Altogether three patients (5\%) underwent another surgical procedure because of haematoma: two cases from the test group and one case from the standard group. Inflammation after the surgical procedure occurred in nine cases (15\%): five cases $(17 \%)$ in the test group and four cases $(13 \%)$ in the standard group.

\section{Hospital stay}

The average postoperative hospital stay was 1.7 days. There was no significant difference in the duration of the hospital stay between the two groups.

\section{Late complications (three and six months after lymphadenectomy)}

Questionnaire about pain was answered by patients three months after lymphadenectomy. Pain was reported by $17 \%$ and $50 \%$ of patients from test and control group ( $p=0.01)$, respectively. Six months after surgical procedure patients from test and control group had neuropathic chronic pain in $20 \%$ and $40 \%(p=0.09)$, respectively. Oedema of the arm was present in 21 patients, 10 from the control group and in 11 from the test group. Limited shoulder movement was present in 14 patients $(23.3 \%), 7$ from each group. Chronic pain after 6 month was present in 18 patients (30\%). There 
were no differences in both arms of our study in frequency of chronic pain after adjuvant treatment (Table 2).

\section{Discussion}

Axillary lymph node dissection is a standard surgical procedure in case of positive lymph nodes in breast cancer or melanoma. ${ }^{19-21}$ Unfortunately, it can cause long-term morbidities: chronic postoperative pain, limited shoulder movement and/ or lymphoedema. ${ }^{5,6}$ Our present prospective randomized study showed that acute pain following breast cancer surgery and axillary lymph node dissection was less frequent and intense after a continuous wound infusion of local anaesthetic compared to a systemic intravenous analgesia with opioids. To our knowledge, there were only six studies dealing with the use of a local anaesthetic for postoperative analgesia in breast carcinoma patients. ${ }^{2,5,9,13,22,23}$ In only three of these studies $^{2,5,9}$, a local anaesthetic was applied to the surgical wound, and only two ${ }^{2,5}$ of these three studies were designed as a prospective randomized trial. Jacobs and Morrison reported the results of a retrospective study, where the wound catheter was connected to an elastomeric pump containing local anesthetic. ${ }^{9}$ They found out that this type of analgesia was safe and reduced postoperative pain. ${ }^{9}$ Talbot et al. performed a prospective, double-blind, randomized, placebo controlled trial on 42 patients after a modified radical mastectomy. ${ }^{2}$ They did not find any difference in analgesia between the treatment group, which received levo-bupivacaine irrigation through the axillary wound drain every four hours for the first 24 hours postoperatively, and the control group, which received irrigation with normal saline. ${ }^{2}$ Instead of perforated catheters, they used axillary drains, which were clamped for 20 minutes every four hours following the application. Thus, a local anaesthetic was not administered continuously. Furthermore, because they did not use perforated catheters, it is questionable if the local anaesthetic irrigated the entire wound area. Our patients with a continuous infusion of local anaesthetic had a lower consumption of opioids and a reduced need for antiemetic drugs compared to the standard opioid-based analgesia group of patients. These results are in accordance with the conclusions of the majority of investigators who studied the role of local anaesthetics in postoperative pain management. 5,9,13,23-29

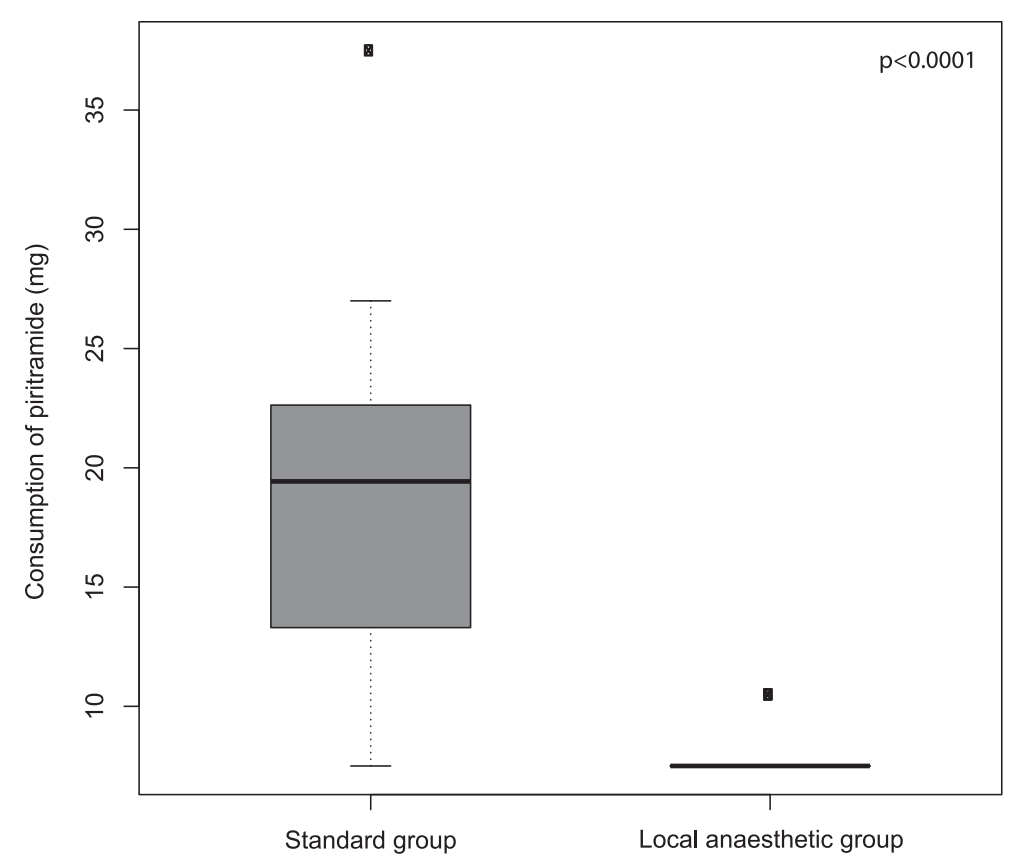

FIGURE 2. Consumption of piritramide during the first 24 hours after the surgical procedure in the local anaesthetic group and in the standard group.

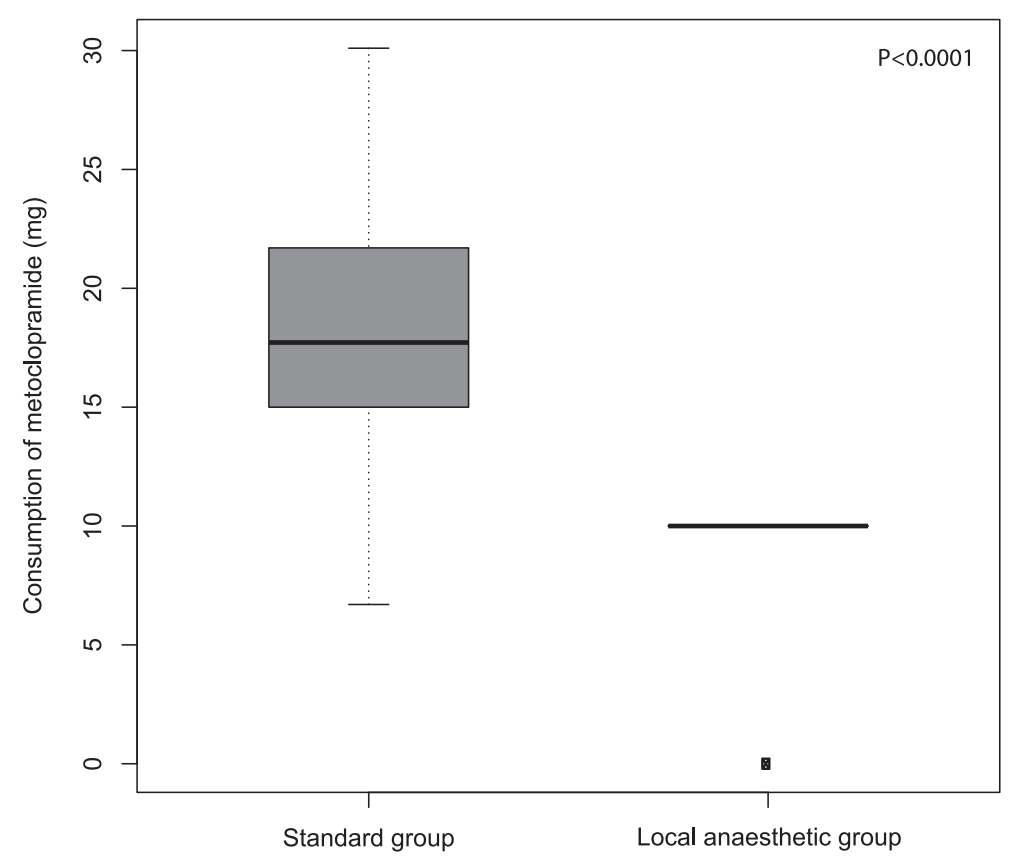

FIGURE 3. Consumption of metoclopramide during the first 24 after the surgical procedure in the local anaesthetic group and in the standard group.

In our study a cost-benefit analysis was not done. The costs of drugs were almost the same in both study arms. So, the cost of treatment with elastomeric pain pump is bigger than standard pain treatment for a price of elastomeric pain pump and perforated catheter, which is 175 Euros 
in our country. But, we proved that patients with a continuous infusion of local anaesthetic had a lower sedation rate compared to the standard opioid-based analgesia group of patients as a consequence of a reduced use of opioids. Almost all patients from the test group had an excellent alertness OAA/S score already on the day of the surgical procedure. Therefore, we believe that a continuous infusion of a local anaesthetic represents a very good pain management option for patients who underwent axillary lymph node dissection and are thus treated as a day case, which has already become a standard of care in some hospitals, for example in the Memorial Sloan-Kettering Cancer Centre in New York. ${ }^{30}$ Painless postoperative patient management and a shorter hospital stay are an essential part of a patient-friendly treatment.

Another very important issue of breast cancer treatment after axillary surgery is the incidence of chronic pain. Many factors are involved in the development of chronic pain after a surgical procedure: genetic susceptibility, psycho-social background, age and gender. ${ }^{31}$ Smith et al. identified age, marital status, employment status and housing as risk factors for post-mastectomy pain syndrome. ${ }^{32}$ Many studies and reviews have noted that a more severe acute postoperative pain is a risk factor for the development of chronic postoperative pain. ${ }^{16}$ It is well known that an infusion of local anaesthetics in the peripheral nerve sheath reduces the incidence of chronic pain after a lower limb amputation ${ }^{33}$, and that an epidural block with a local anaesthetic before the surgery reduces longterm post-thoracotomy pain. ${ }^{34}$ However, the use of a local anaesthetic immediately after the surgical procedure to prevent the post-mastectomy pain syndrome is still not sufficiently defined. Three months after lymphadenectomy, our patients on postoperative continuous infusion of local anaesthetic reported pain less often than patients on opioid-based analgesia ( $17 \%$ vs. $50 \%$; p $=0.01)$.

However, six months after lymphadenectomy there was just a trend for lower pain in continuous infusion of local anaesthetic group of patients in comparison to opioid-based analgesia (20\% vs. $40 \%$; $p=0.09$ ). Similar outcome on occurrence of post-mastectomy pain syndrome was reported by Fassoulaki et al. ${ }^{13,22}$ In their first study, the anaesthetic cream was applied locally on the skin just before and at the end of the surgical procedure, and daily thereafter for a total of four days. The incidence of pain three months after surgical procedure was $43 \%$ in the study group and $91 \%$ in the placebo group $(p=0.002) .{ }^{22}$ In their second study, the incidence of pain after three months was $45 \%$ in the treatment group (local anaesthetic cream, irrigation of brachial plexus and intercostal places with a local anaesthetic and gabapentin tablets) versus $82 \%$ in the placebo group $(\mathrm{p}=0.028)$. They reported that chronic pain after six months in the treatment group $(30 \%)$ was less common than in the placebo group (57\%), but the difference was not significant. Obviously, a local anaesthetic applied at the time of a surgical procedure can affect the frequency and intensity of pain in subacute phase, but it is questionable if it can affect chronic post-mastectomy pain syndrome. In our study, the complication rate after a surgical procedure did not differ in patients treated with a continuous infusion of a local anaesthetic through the wound catheter and those who received a standard intravenous opioid analgesia. The experience of other authors who used local anaesthetics is similar. The rates of inflammation or hematomas were not higher after the use of local anaesthetics compared to the placebo or standard analgesia treatment gro ups. $2,5,9,13,15,24-26,28,29,33,34$ On the contrary, local anaesthetics seem to reduce the occurrence of inflammation. ${ }^{35,36,37}$ Like other authors, we also did not notice any toxic side effects of local anaesthetics. In our study, we used $0.25 \%$ levo-bupivacaine, which was reported as one of the safest local anesthetics. ${ }^{38}$

To conclude, our prospective randomized study confirmed that the application of a wound catheter with an elastomeric pump with a local anaesthetic is safe, easy and effective for reducing acute postoperative pain. Continuous infusion of a local anaesthetic into the wound reduces opioid consumption and results in less postoperative sedation and a reduced need for antiemetic drugs. The elastomeric pump is comfortable for both the patients and a nursing stuff. Thus, the patients are more alert, do not feel pain and, consequently, do not need intensive monitoring and nursing care.

\section{References}

1. Merskey H, Bogduk N, editors. Classification of chronic pain: descriptions of chronic pain syndromes and definitions of pain terms. Second edition. Seattle: IASP Press; 1994

2. Talbot $H$, Hutchinson P, Edbrooke DL, Wrench I, Kohlhardt SR. Evaluation of a local anaesthesia regimen following mastectomy. Anaesthesia 2004; 59: 664-7.

3. Katz J, Poleshuck EL, Andrus CH, Hogan LA, Jung BF, Kulick DI, et al. Risk factors for acute pain and its persistence following breast cancer surgery. Pain 2005; 119: 16-25.

4. Almeida TF, Roizenblatt $S$, Tufik S. Afferent pain pathways: a neuroanatomical review. Brain Research 2004; 1000: 40-56. 
5. Schell SR. Patient outcome after axillary lymph node dissection for breast cancer: use of postoperative continuous local anaesthesia infusion. J Surg Res 2006; 134: 124-32

6. Lahajnar CS. [Pain after the surgical treatment in breast cancer patients at the Institute of Oncology Ljubljana.] [Slovenian]. Onkologija 2007; 2: 114-8.

7. Tasmuth T, von Smitten K, Hietaneon P, Kataja M, Kalso E. Pain and other symptoms after different treatment modalities of breast cancer. Ann Oncol 1995; 6: 453-9.

8. Warmuth MA, Bowen G, Prosnitz LR, Chu LO, Broadwater G, Peterson B, et al. Complications of axillary lymph node dissection for carcinoma of the breast: A report based on a patient survey. Cancer 1988; 83: 1362-8.

9. Jacobs VR, Morrison JE Jr. Application of a locally placed anaesthesia catheter for reduction of postoperative pain after mastectomy for breast cancer. Int J Fertil Womens Med 2006; 51: 225-9.

10. Lubenow TR, Ivankovich AD, McCarthy RJ. Management of acute postoperative pain. In: Barash PG, Cullen BF, Stoelting RK, editors. Clinical Anesthesia. 3rd edition. Philadelphia: Lippincott-Rawen; 1997. p. 1305-37.

11. White PF. The role of non-opioid analgesic techniques in the management of pain after ambulatory surgery. Anesth Analg 2002; 94: 577-85.

12. White PF. The changing role of non-opioid analgesic techniques in the management of postoperative pain. Anesth Analg 2005; 101: S5-22.

13. Fassoulaki A, Triga A, Melemeni A, Sarantopoulos C. Multimodal analgesia with gabapentin and local anesthetics prevents acute and chronic pain after breast surgery for cancer. Anesth Analg 2005; 10: 1427-32.

14. Borgeat A, Rawal N, editors. Wound catheter techniques for postoperative analgesia. Cardiff: Darwin Grey Communications; 2008.

15. Rawal N, Axelsson K, Hylander J, Allvin R, Amilon A, Lidegran G, Hallén J. Postoperative patient-controlled local anesthetic administration at home. Anesth Analg 1998; 86: 86-9.

16. Liu SS, Kehlet H. Clinical approach to the patient with postoperative pain. ACS surgery: principles and practice. Hamilton: BC Decker Inc.; 2009.

17. Kay B. A clinical investigation of piritramide in the treatment of postoperative pain. Brit J Anesth 1971; 43: 1167-71.

18. Chernik DA, Gillings D, Laine $H$, Hendler J, Silver JM, Davidson AB, et al. Validity and Reliability of the Observer's Assessment of Alertness/Sedation Scale: study with intravenous midazolam. J Clin Psychpharmacol 1990; 10(4): 244-51.

19. Pilko G, Besic N, Zgajnar J, Hocevar M. Prognostic heterogeneity after the excision of lymph node metastases in patients with cutaneous melanoma. Surg Oncol 2011; 20: 26-34

20. Strojan P. Role of radiotherapy in melanoma management. Radiol Oncol 2010; 44: 1-12.

21. Adamczyk B, Murawa D, Połom K, Spychała A, Nowaczyk P, Murawa P. Preliminary experience in sentinel node and occult lesion localization (SNOLL) technique - One center study. Rep Pract Oncol Radiother 2011; 16: $221-6$

22. Fassoulaki A, Sarantopoulos C, Mlemeni A, Hogan Q. EMLA reduces acute and chronic pain after breast surgery for cancer. Reg Anesth Pain Med 2000; 25: 350-5.

23. Fassoulaki A, Sarantopoulos C, Melemeni A, Hogan Q. Regional block and mexiletine: the effect on pain after cancer breast surgery. Reg Anesth Pain Med 2001; 26: 223-8.

24. Gottschalk A, Burmeister MA, Radtke P, Krieg M, Farokhzad F, Keissl S, et al. Continuous wound infiltration with ropivacaine reduces pain and analgesic requirement after shoulder surgery. Anesth Analg 2003; 97: 1086 -91.

25. Vintar N, Rawal N, Veselko M. Intraarticular patient-controlled regional anesthesia after artroscopically assisted anterior cruciate ligament reconstruction: Ropivcaine/Morphine/Ketrorolac versus Ropivacaine/Morphine. Anesth Analg 2005; 101: 573-8.

26. Beaussier $M$, El'Ayoubi $H$, Schiffer $E$, Rolling $M$, Parc $Y$, Mazoi JX, et al. Continuous preperitoneal infusion of ropivacaine provides effective analgesia and accelerates recovery after colorectal surgery: a randomized, double-blind, placebo-controlled study. Anesthesiology 2007; 107: 461-8.

27. Vintar N, Pozlep G, Rawal N, Godec M, Rakovec S. Incisional self-administration of bupivacaine or ropivacaine provides effective analgesia after inguinal hernia repair. Can J Anaesth 2002; 49: 481-6.
28. Fredman B, Shapiro A, Zohar E, Feldman E, Shorer S, Rawal N, et al. The analgesic efficacy of patient-controlled ropivacaine instillation after Cesarean delivery, Anesth Analg 2000; 91: 1436-40.

29. White PF, Rawal S, Latham P. Use of a continuous local anesthetic infusion for pain management after median sternotomy. Anaesthesiology 2003; 99: 918-23.

30. Barry M, Weber WP, Lee S, Mazzella A, Sclafani LM. Enhancing the clinical pathway for patients undergoing axillary lymph node dissection. Breast 2012; 21: 440-3.

31. Kehlet $\mathrm{H}$, Jensen TS, Woolf $\mathrm{C}$. Persistent postsurgical pain: risk factors and prevention. Lancet 2006; 367: 1618-25.

32. Smith WC, Bourne D, Squair J, Phillips DO, Chambers WA. A retrospective cohort study of post mastectomy pain syndrome. Pain 1999; 83: 91-5.

33. Fisher A, Meller Y. Continuous postoperative regional analgesia by nerve sheath block for amputation surgery - a pilot study. Anesth Analg 1991; 72: $300-3$

34. Obata H, Saito S, Fujina N, Fuse Y, Ishizaki K, Goto F. Epidural block with mepivacaine before surgery reduces long-term post-thoratcotomy pain. Can $J$ Anesth 1999; 46: 1127-32.

35. Sakuragi T, Ishino H, Dan K. Bacatericidal activity of clinically used local anesthetics on Staphylocossus aureus. Reg Anesth 1996; 21: 239-42.

36. Stratford AF, Zoutman DE, Davidson JS. Effect of lidocaine and epinephrine on Staphylococcus aureus in a guinea pig model of surgical wound infection. Plast Reconstr Surg 2002; 110: 1275-9.

37. Parr AM, Zoutman DE, Davidson JS. Antimicrobial activity of lidocaine against bacteria associated with nosocomial wound infection. Ann Plast Surg 1999; 43: 239-45.

38. Mather LE, Copeland SE, Ladd LA. Acute toxicity of local anesthetics: underlying pharmacokinetic and pharmacodynamic concepts. Reg Anesth Pain Med 2005; 30: 553-66. 


\title{
Uterine perforation - 5-year experience in 3-D image guided gynaecological brachytherapy at Institute of Oncology Ljubljana
}

\author{
Barbara Segedin ${ }^{1}$, Jasenka Gugic ${ }^{1}$, Primoz Petric ${ }^{1,2}$ \\ ${ }^{1}$ Institute of Oncology Ljubljana, Department of Radiotherapy, Ljubljana, Slovenia \\ ${ }^{2}$ National Center for Cancer care and Research Doha, Qatar
}

Radiol Oncol 2013; 47(2): 154-160.

Received 19 November 2012

Accepted 1 March 2013

Correspondence to: Barbara Šegedin, MD, Institute of Oncology Ljubljana, Department of Radiotherapy, Zaloška 2, SI-1000 Ljubljana, Slovenia. E-mail: bsegedin@onko-i.si

Disclosure: No potential conflicts of interests were disclosed.

Background. Accurate applicator placement is a precondition for the success of gynaecological brachytherapy (BT). Unrecognized uterine perforation can lead to bleeding, infection, high doses to pelvic organs and underdosage of the target volume, resulting in acute morbidity, long-term complications and reduced chance of cure. We aimed to assess the incidence and clinical characteristics of our cases with uterine perforation, review their management and impact on the treatment course.

Patinets and methods. In all patients, treated with utero-vaginal image guided BT for gynaecological cancer between January 2006 and December 2011, the CT/MR images with the applicator in place were reviewed. The incidence of uterine perforations was recorded. Clinical factors that may have predisposed to increased risk of perforation were recorded. Management of perforations and their impact on treatment course was assessed.

Results. 219 patients (428 applications) were suitable for analysis. Uterine perforation was found in 13 (3.0\%) applications in $10(4.6 \%)$ patients. The most frequent perforation site was posterior uterine wall $(n=9)$, followed by anterior wall $(n=2)$ and fundus $(n=2)$. All cases were managed conservatively, without complications. Prophylactic antibiotics were administered in 8 cases. In 4 patients, abdominal and/or transrectal ultrasound (US) guidance was used on subsequent applications for applicator insertion; adequate applicator placement was achieved and treatment completed as planned in all cases.

Conclusions. 3D imaging for BT planning enables accurate identification of uterine perforations. The incidence of perforations at our department is one of the lowest reported in the literature. US guidance of applicator insertion is useful and feasible, allowing to complete the planned treatment even in challenging cases.

Key words: uterine perforation; brachytherapy; 3D imaging; ultrasound guidance

\section{Introduction}

Combination of external beam radiotherapy, concomitant chemotherapy and brachytherapy (BT), is an essential treatment modality for locally advanced gynaecological cancer. In intracavitary (IC) BT of cervix cancer, an applicator (uterine tandem and vaginal ovoids, cylinder or ring) is placed in the uterine cavity and vaginal fornices in general or spinal anaesthesia. In the definitive treatment of endometrial cancer, Heyman capsules are inserted into the uterus. Intraoperative complications of IC BT include vaginal lacerations, penetration of the tandem into the uterine wall and perforation of the uterus and other pelvic organs with the applicator. ${ }^{1-3}$ Reported incidence of uterine perforations by an IC BT applicator ranges from $1.75 \%$ to $13.7 \% .^{1,4-6}$ The most common site of perforations, cited in the literature, is uterine fundus. ${ }^{4}$ Patients over 60 years are particularly predisposed to uterine perforation, probably due to vaginal atrophy and anatomical distortion of the cervix..$^{2,3,6}$ Uterine perforation oc- 
curs more frequently in patients with anatomical distortions of the cervix and/or cervical stenosis due to advanced disease, post irradiation fibrosis and previous cone biopsy. ${ }^{1,3,4}$ Other predisposing factors include retroverted/retroflexed and extremely anteverted/anteflexed uterus. ${ }^{6-8}$

Most cases of uterine perforations resolve without squeals after conservative treatment. Nevertheless, infection, haemorrhage or peritoneal tumour-cell seeding may occur. ${ }^{1,9}$ Uterine perforations therefore require specific attention from the medical team, including the radiation oncologist, gynaecologist and anaesthesiologist. An important potential consequence of an unrecognized uterine perforation is a high level of uncertainty regarding the dose distribution in the tissues around the incorrectly inserted IC applicator. Accurate positioning of the IC applicator is critical for delivering appropriate doses of irradiation to the target volume while keeping the doses to the surrounding organs at risk below their tolerance limits. Consequently, unrecognized uterine perforation can lead to under dosage of the target volume, compromising local control probability. ${ }^{10}$ In addition, the perforating applicator may come in direct contact or in vicinity of the organs at risk, leading to their exposure to excessive doses, resulting in acute and long-term gastrointestinal and genitourinary complications.

At the Institute of Oncology Ljubljana, we have been using 3D MRI/CT - assisted BT for gynaecological malignancies since 2006. ${ }^{11-18}$ The main advantages of this approach over conventional $2 \mathrm{D}$ radiography-based techniques ${ }^{19}$ include: (1) the ability for individualized treatment tailoring, based on patients anatomy, tumour size and topography, (2) analysis of the dose volume histogram parameters and (3) straightforward recognition of applicator misplacement, including the uterine perforations.

The main objective of the present study was to determine the incidence and characteristics of uterine perforations at our department between January 2006 and December 2011 in the era of 3D MRI/CT - assisted BT. We evaluated the characteristics of the patients presenting with perforation and reviewed their management and impact of the perforations on the treatment course.

\section{Patients and methods}

\section{Patients}

All patients, treated with utero-vaginal 3D image based BT at our department for gynaecological cancer between January 2006 and December 2011, were included in analysis, irrespective of the treatment intent (curative vs. palliative). Pelvic MRI and/or CT images from each insertion with the applicator in place were reviewed retrospectively to find the incidence and location of uterine perforations. Patients' medical records were studied to evaluate their management and assess the impact on the treatment course. Clinical factors, known to predispose to an increased risk of perforation, were recorded.

\section{Treatment}

Our general treatment approach, as applied in the cases of radiotherapy with curative intent, was described elsewhere in detail. ${ }^{11-15}$ In palliative treatments, judicious individualisation of treatment techniques and dose fractionation schedules was applied. However, the brachytherapy technique did not differ considerably from the method used in curative treatment, as described below.

3D conformal CT based external beam radiotherapy (EBRT) was delivered at a 15 MV linear accelerator with a 4-field box technique, planning to apply a total dose of 45 to 50.4 Gy (1.8-2 Gy per fraction, five fractions per week) to the whole pelvis. Prophylactic paraaortic or inguinal irradiation was planned in selected patients. A boost to bring the total EBRT dose up to 60-65 Gy was planned for macroscopically involved metastatic lymph nodes. Concomitant chemotherapy (weekly cisplatin, $40 \mathrm{mg} / \mathrm{m}^{2}$ ) was considered in all cervix cancer patients. Following EBRT, 1-3 BT applicator insertions were scheduled in weeks 6 and 7 of treatment. Prior to each insertion, gynaecological examination was carried out by the radiation oncologist. Application technique was individually adapted to each patient at each insertion, based on the clinical and radiological findings. The insertion was carried out with the patient in subarachnoid, general or paracervical anaeasthesia, placed in lithotomy position. All applications were performed by a radiation oncologist alone, except in 3 cases, where the procedure was carried out in cooperation with a gynaecologic oncologist. Following dilatation of the cervical canal, a CT or MRI-compatible applicator was inserted into the uterus and vaginal fornices. In cervix cancer patients, a plastic or metallic tandem was used as the intrauterine part. In endometrial cancer, plastic Heyman capsules or metallic Rotte Y-applicator were applied. For other tumour sites, the decision on the intrauterine part was individual. In selected patients, a combined intracavitary/interstitial (IS) application was performed, using a ring template-cap for guidance of 


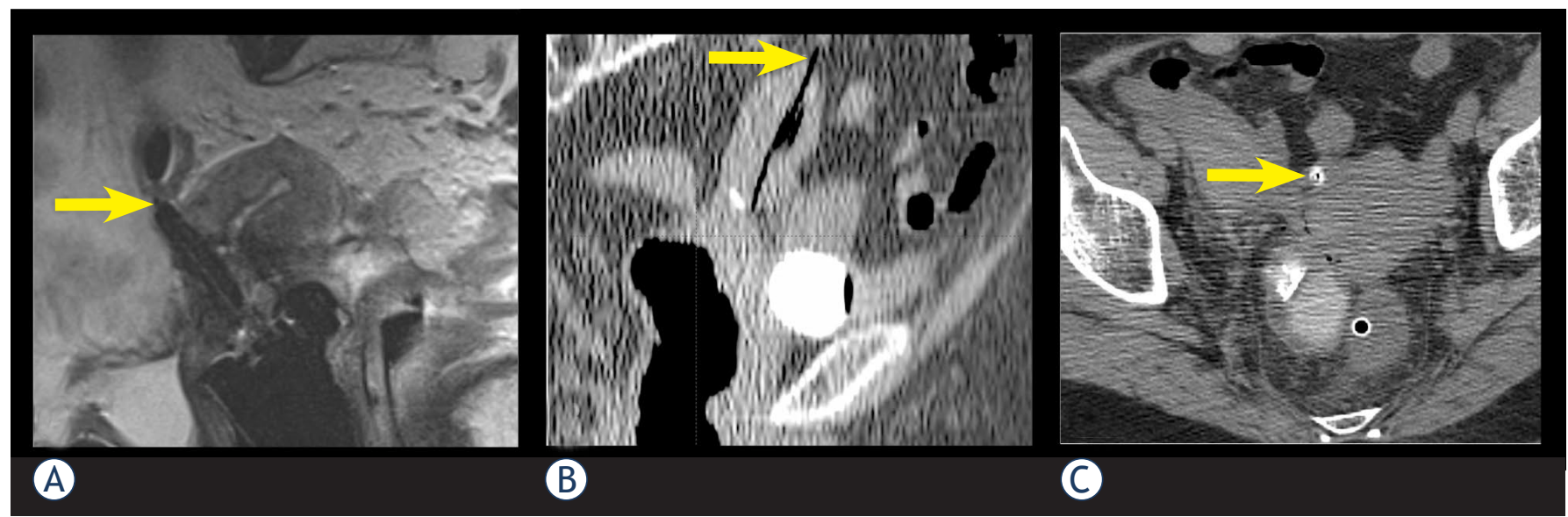

FIGURE 1. The sites of the uterine perforations. White arrows point at a perforation of posterior uterine wall (A), uterine fundus (B) and anterior uterine wall (C) by an IU applicator (tandem or Heyman capsule).

plastic needles into the parametria ${ }^{20-22}$ or by freehand transvaginal insertion. Ultrasound guidance was used to aid applicator placement in challenging situations. Vaginal packing was used to fix the applicator position. Following the insertion, the patients were transported to the MRI or CT scanner. The paradigm of our BT dose prescription and treatment planning practice is represented by the image guided adaptive BT of cervix cancer and was described elsewhere. ${ }^{11-18}$

\section{Results}

\section{Incidence and characteristics of perforations}

From January 2006 to December 2011, 496 uterovaginal applicator insertions were performed in 253 patients. After exclusion of 34 patients with plain radiography based BT, 428 applications in 219 patients were left for analysis.

Median patient age was 62 years (range 31 to 88), $126(57.5 \%)$ of them were older than 60 years. The most common tumour site was cervix (172 patients; $78.5 \%)$. FIGO stage distribution of the cervix cancer patients was as follows: stage I in 21, stage II A or B in 69, stage III A or B in 67 and stage IV A in 15 cases. Thirty nine $(17.8 \%)$ patients were treated for endometrial cancer (FIGO stage distribution: stage I in 14, stage II in 3, stage III in 4 and stage IV in 1 case; in 17 cases FIGO stage had not been defined). Four patients $(1.8 \%)$ were treated for vaginal carcinoma, two $(0.9 \%)$ for recurrent ovarian cancer with palliative intent and two $(0.9 \%)$ for atypical endometrial hyperplasia.
One hundred and nineteen out of 219 patients $(54.3 \%)$ underwent 2 BT insertion procedures. The maximum number of insertions was 3 in $48(21.9 \%)$ patients. Tandem - ring applicator was used in 329 (76.9\%), tandem - ovoids applicator in $25(5.8 \%)$ and Heyman capsules in 74 (17.3\%) applications.

Uterine perforation was identified in $13(3.0 \%)$ applications in $10(4.6 \%)$ patients. Median age of patients, presenting with perforation, was 74 years (range 51-83 years). All patients with perforation, except two, were older than 60 years. Seven $(70 \%)$ patients were treated for cervical cancer, two $(20 \%)$ for endometrial and one (10\%) for ovarian cancer. Five patients were treated with palliative intent. Posterior uterine wall was the most common site of perforation with $9(70 \%)$ cases, followed by the anterior wall and fundus with $2(15 \%)$ cases each (Figure 1). In most cases, one or more of the characteristics, previously described as the predisposing factors to uterine perforation (necrotic cervical tumour, cervical polyp, submucosal myoma, stenosis or distortions of cervical canal, especially due to prior conization, retroflexed or extremely anteflexed uterus) was found. ${ }^{1-4,6}$ The most commonly identified risk factor was age over 60 years in $8(80 \%)$, followed by necrosis and distortion of cervical canal in $4(40 \%)$ cases. Table 1 summarizes the frequency of these characteristics in individual patients, presenting with uterine perforation.

\section{Management and impact on the treatment course}

In all cases where a perforation was identified on post-insertion imaging, the applicator was re- 
moved and the patient treated conservatively. General condition, blood pressure and blood count were monitored for a minimum of 24 hours. Prophylactic antibiotics, in most cases dual prophylaxis with ciprofloxacin and metronidazol, were administered in $8(62 \%)$ patients. Three $(20 \%)$ patients received blood transfusion due to a drop in haemoglobin level (median 22g/L (15-26)). All patients with uterine perforation that were treated with definitive irradiation with curative intent, completed their treatment as planned. In $4(40 \%)$ patients, transabdominal and/or transrectal ultrasound guidance was used in subsequent application(s) and in three (30\%), the subsequent application(s) were performed by a gynaecologic oncologist. At subsequent application, re-perforation occurred in three patients (without the use of ultrasound guidance) and was followed by a successful reposition of the applicator to an appropriate position under same anaesthesia in one patient. No other major intraoperative complications occurred in our series. The incidence of vaginal lacerations was not systematically recorded.

At the time of the last follow up there were no signs of intraperitoneal tumour-cell seeding in any of the patients in whom uterine perforation occurred.

\section{Discussion}

Age over 60 years, anatomical distortion of the cervix, cervical stenosis, especially due to prior cone biopsy and variations of the physiological position of the uterus have already been reported as risk factors for uterine perforation in the literature. ${ }^{1-4,6}$ In our group of patients, age over 60 was the most common identified risk factor; most of the patients had at least one additional risk factor.

Corn et al. analysed whether the technical qualities of a BT application have an impact on the outcome of patients with locally advanced cervix cancer treated by definitive irradiation. ${ }^{10}$ The analysis was restricted to patients having only one intracavitary application to avoid potential interference effect of subsequent implants, which could compensate the presumed negative impact of a technically suboptimal first application. Plain radiographs of 66 patients were reviewed by a radiation physicist and a radiation oncologist with expertise in gynaecologic radiotherapy. On the basis of assessment of four parameters, the applications were scored as »ideal«, »unacceptable« or »adequate«. Significantly improved 5-year local control was seen when comparing ideal and adequate placement to unacceptable placement $(68 \%$ vs. $34 \%$, p $=$ 0.02 ). A strong trend toward improved 5-year survival was also noted among the group with ideal and adequate placement as opposed to unacceptable placement $(61 \%$ vs. $42 \%, \mathrm{p}=0.13)$. This study demonstrates direct influence of competent technical implant performance on local tumour control and even on survival.

Insertion of the intrauterine tandem or Heyman capsules at the time of IC BT procedure has traditionally been performed by relying only on the experience of the operating physician, the clinical assessment of the insertion adequacy and the postoperative 2D imaging - plain radiography. Specific radiological criteria, based on assessment of two orthogonal pelvic radiographs with the applicator in place were proposed to identify cases at risk of a misplaced applicator. ${ }^{7}$ However, notwithstanding the importance of clinical evaluation and the experience accumulated with conventional radiography-based techniques, this approach has some inherent limitations. Conventional radiography can only show the applicator position relative to the bony structures, not to the adjacent organs, even the uterus itself. Consequently, it can be expected that the techniques that employ only the clinical evaluation and plain radiography may underestimate the incidence of uterine perforations. Accordingly, the published results on the use of postoperative plain radiography show that even in case where tandem position is considered ideal according to 2D radiological criteria, 3D imaging reveals uterine perforation in approximately $3-10 \%{ }^{23,24}$ Recent review of medical records of the patients treated at our department from 2001 to 2005 with IC +/- IS BT for gynaecological tumours revealed only 1 clinically suspicious perforation (unpublished results). We can assume that this underestimates the true incidence of uterine perforations at our department during that period when only conventional 2-D radiography was used for BT planning.

Over past decades a variety of techniques have been described to determine the position of the tandem and to identify uterine perforation. Pelvic pneumography was proposed, but was soon abandoned, because it was technically too demanding. ${ }^{25}$ Hysterography with contrast application through a modified tandem was proposed, but fell out of favour due to the potential risk of dissemination of tumour cells into the pelvic cavity. ${ }^{26}$ Matsuyama et al. proposed attachment of surgical haemostatic clips in the uterine serosa during laparotomy in order to visualise the outer contour of the uterus on 
TABLE 1. Presence of the known risk factors in individual patients and tumours in which uterine perforation occurred

\begin{tabular}{|c|c|c|c|c|c|c|c|c|c|c|}
\hline \multirow[t]{2}{*}{ Risk factor } & \multicolumn{10}{|c|}{ Patient presenting with uterine perforation } \\
\hline & 1 & 2 & 3 & 4 & 5 & 6 & 7 & 8 & 9 & 10 \\
\hline Age $>60$ y & - & - & • & - & • & & • & - & - & \\
\hline Necrosis & & & • & & & - & & - & & - \\
\hline Cervical polyp & & & & & • & & & & & \\
\hline Myoma & & - & & & & & & & - & \\
\hline RF uterus & & & & - & & & & . & • & \\
\hline Conization & & & & - & & & & & & \\
\hline
\end{tabular}

$y=$ years; $C C=$ cervical canal; $R F=$ retroflected

plain radiographs. ${ }^{5}$ The need for surgery and also the possibility to underestimate the incidence of uterine perforation led to the abandonment of the technique. ${ }^{4}$ Laparoscopic evaluation is also used, but it is now reserved only for rare cases in which 3D imaging techniques are not suitable or do not give enough information about tandem position. ${ }^{7}$

3D imaging techniques are superior to the above listed approaches, because they allow for a direct, non-invasive and unequivocal visual assessment of the tandem position and identification of eventual uterine perforation. They allow for an excellent visualization of the tandem and surrounding structures and also the relationship of the tandem to these structures. These imaging techniques are performed post-insertion with the main purpose of 3D optimization of the dose distribution. Detection of the uterine perforation by using CT or MRI requires removal of the tandem and another attempt of insertion, which consequently delays the treatment and requires anaesthesia. If the procedure is conducted in spinal anaesthesia, it is in some cases possible to correct the position of the tandem in the same anaesthesia session. Repeated imaging (CT/ $M R I)$ is required for verification of applicator position and treatment planning, delaying the procedure and increasing the cost.

Recently, 3D image guided BT has been implemented at a growing number of institutions worldwide, tailoring the dose distribution individually, according to the patient's patho-anatomical situation. Favourable reports on dosimetric outcome of the 3D techniques are reflected in encouraging clinical results. ${ }^{13,27-32}$ However, to our knowledge, the value of $3 \mathrm{D}$ imaging in identifying the cases of uterine perforations in BT has not been addressed so far. Uterine perforation was found in 13 (3.0\%) applications in our series. By using 3D imaging, these cases were identified immediately following applicator insertion, allowing taking appropriate medical measures on time. In this way, the risk of potentially serious sequels of the perforation could be avoided in all patients and curative treatment could be completed as planned.

Ultrasound allows for a good visualization of the intrauterine applicator, uterus and urinary bladder, and can, consequently, demonstrate uterine or urinary bladder perforation. Importantly, ultrasound can be performed either postoperatively or intraoperatively as on-line guidance of the insertion. ${ }^{433-35}$ Granai et al. reported on the use of postoperative ultrasound in 50 consecutive applications (28 patients). In 17 applications (34\%), the tandem was found to be suboptimally positioned. In 12 (24\%) applications, it penetrated the myometrium and in $5(10 \%)$ cases it frankly perforated the uterine wall. In all cases where postoperative ultrasound identified the applicator malposition, clinical and radiographic assessment indicated accurate placement. In a report on the use of intraoperative ultrasound in 73 consecutive applications, ultrasound allowed direct visualization of the procedure and accurate tandem positioning in 72 cases. ${ }^{4}$ Consistent with findings of other authors, intraoperative transabdominal ultrasound was identified as a useful adjunct at the time of IC BT, allowing to complete the planned treatment even in difficult cases. ${ }^{35,36}$ According to Davidson et al., the use of transabdominal US influenced the length and angle of IU tandem chosen in $49 \%$ of patients. ${ }^{24}$ They also report on shortening of the applicator insertion time and fewer requests for assistance of a gynaecologist at the procedure. Fleischer et al. reported on use of transrectal sonography (TRUS) at 
the time of IC BT and also for some other gynaecological procedures. ${ }^{37}$ They found transrectal ultrasound to be useful in providing guidance for these procedures and at the same time helpful in avoiding uterine perforation and urinary bladder injury. Sharma et al. published a series of 40 gynecological BT procedures performed with TRUS guidance. ${ }^{38}$ In all cases, adequate position of IU tandem was achieved, while the procedure time was not prolonged with the use of TRUS. In our experience, the use of intraoperative US provides immediate feedback information and enables a guided application. At our department, intraoperative, mainly transrectal ultrasound guidance of insertion is used in challenging cases. In the present study, transabdominal and/or transrectal ultrasound was used in $4(31 \%)$ patients with prior uterine perforation, and enabled successful insertion in all cases. Importantly, corrective measures can be made immediately and therefore there is no need for an additional anaesthesia. Moreover, ultrasound is widely available, feasible and associated with low costs compared to other imaging modalities.

\section{Conclusions}

3D imaging techniques, used for BT planning, not only allow for the treatment optimisation, but also enable accurate identification of uterine perforations, preventing potential acute and chronic complications, including treatment failure due to underdosage of the tumour and overdosage of the organs at risk. Transrectal or transabdominal intraoperative ultrasound guidance is a very useful tool at the time of IC BT and generally allows for an appropriate positioning of the applicator, even in difficult cases. Special care is warranted when performing BT applications in elderly patients in the presence of known predisposing factors for uterine perforation. In such cases, as well as in patients with prior perforation, US guidance is recommended.

\section{References}

1. Kim RY, Levy DS, Brascho DJ, Hatch KD. Uterine perforation during intracavitary application. Prognostic significance in carcinoma of the cervix. Radiology 1983; 147: 249-51.

2. Corn BW, Shaktman BD, Lanciano RM, Hogan WM, Cater JR, Anderson L, et al. Intra- and perioperative complications associated with tandem and colpostat application for cervix cancer. Gynecol Oncol 1997; 64: 224-9.

3. Jhingran A, Eifel PJ. Perioperative and postoperative complications of intracavitary radiation for FIGO stage I-III carcinoma of the cervix. Int J Radiat Oncol Biol Phys 2000; 46: 1177-83.
4. Granai CO, Doherty F, Allee P, Ball HG, Madoc-Jones H, Curry SL. Ultrasound for diagnosing and preventing malplacement of intrauterine tandems. Obstet Gynecol 1990; 75: 110-3.

5. Matsuyama T, Tsukamoto N, Matsukuma K, Kamura T, Jingu K. Uterine perforation at the time of brachytherapy for the carcinoma of the uterine cervix. Gynecol Oncol 1986; 23: 205-11.

6. Barnes EA, Thomas G, Ackerman I, Barbera L, Letourneau, Lam K, et al. Prospective comparison of clinical and computed tomography assessment in detecting uterine perforation with intracavitary brachytherapy for carcinoma of the cervix. Int J Gynecol Cancer 2007; 17: 821-6.

7. Irvin W, Rice L, Taylor P, Anderson W, Schneider B. Uterine perforation at the time of brachytherapy for carcinoma of the cervix. Gynecol Oncol 2003; 90: 113-22.

8. Milman RM, Goodman P. Uterine perforation by an intracavitary tandem: CT demonstration. Clin Imaging 1991; 15: 223-6.

9. Mills SE, Sugg NK, Mahnesmith RC. Endometrial carcinoma with pelvic involvement following uterine perforation. Diagn Gynecol Obstet 1981; 3: 149-54.

10. Corn BW, Hanlon AL, Pajak TF, Owen J, Hanks GE. Technically accurate intracavitary insertions improve pelvic control among patients with locally advanced carcinoma of the uterine cervix. Gynecol Oncol 1994; 53: 294-300.

11. Petric P, Hudej R, Marolt-Music M. MRI-based brachytherapy for cervical cancer. Onkologija 2008; 12: 46-51.

12. Petric P. Moving from 2D to 3D brachytherapy in gynecological tumors. $J$ Contemp Brachyther 2011; 3: 161-2.

13. Petric P, Hudej R, Segedin B, Zobec Logar HB. MRI assisted treatment planning improves the DVH parameters in cervix cancer brachytherapy. Radiother Oncol 2011; 99: 264-5.

14. Petric P, Hudej R, Rogelj P, Blas M, Segedin B, Zobec Logar HB, et al. Comparison of 3D MRI with high sampling efficiency and 2D multiplanar MRI for contouring in cervix cancer brachytherapy. Radiol Oncol 2012; 46:242-51.

15. Petric P, Dimopoulos JCA, Kirisits C, Berger D, Hudej R, Pötter R. Inter- and intraobserver variation in HR-CTV contouring: intercomparison of transverse and paratransverse image orientation in 3D-MRI assisted cervix cancer brachytherapy. Radiother Oncol 2008; 89: 164-71.

16. Haie-Meder C, Pötter R, Van Limbergen E, Briot E, De Brabandere M, Dimopoulos JCA, et al. Recommendations from Gynaecological (GYN) GEC-ESTRO Working Group (I): concepts and terms in 3D image based 3D treatment planning in cervix cancer brachytherapy with emphasis on MR assessment of GTV and CTV. Radiother Oncol 2005; 74: 235-45.

17. Pötter R, Haie-Mader C, Van Limbergen E, Barillot I, De Brabandere M, Dimopoulos J, et al. Recommendations from gynaecological (GYN) GEC ESTRO working group (II): concepts and terms in 3D image-based treatment planning in cervix cancer brachytherapy-3D dose volume parameters and aspects of 3D image-based anatomy, radiation physics, radiobiology. Radiother Oncol 2006; 78: 67-77.

18. Petric P, Pötter R, Van Limbergen E, Haie-Meder C. Adaptive contouring of the target volume and organs at risk. In: Viswanathan AN, Kristis C, Erickson $\mathrm{BE}$, Pötter R, editors. Gynecologic radiation therapy, novel approaches to image-guidance and management. Berlin Heidelberg: Springer Verlag; 2011. p. 99-118.

19. Gerbaulet A, Pötter R, Haie-Meder C. Cervix cancer. In: Gerbaulet A, Pötter $\mathrm{R}$, Mazeron JJ, Meertens $\mathrm{H}$, Van Limbergen E, editors. The GEC ESTRO handbook of brachytherapy. Brussels: European Society of Therapeutic Radiology and Oncology; 2002. p. 301-63.

20. Petric $P$, Hudej R, Marolt-Music M. MRI assisted cervix cancer brachytherapy pre-planning, based on insertion of the applicator in para-cervical anaesthesia: preliminary results of a prospective study. J Contemp Brachyther 2009; 1: 163-9.

21. Dimopoulos JCA, Kirisits C, Petric P, Georg P, Lang S, Berger D, et al. The Vienna applicator for combined intracavitary and interstitial brachytherapy of cervical cancer: clinical feasibility and preliminary results. Int J Radiat Oncol Biol Phys 2006; 66: 83-90.

22. Petric P, Hudej R, Rogelj P, Lindegaard J, Tanderup K, Kirisits C, et al. Frequency-distribution mapping of HR CTV in cervix cancer: possibilities and limitations of existent and prototype applicators. Radiother Oncol 2010; 96: 70 
23. Makin WP, Hunter, RD. CT scanning in intracavitary therapy: Unexpected findings in "straightforward" insertions. Radiother Oncol 1988; 13: 253-5.

24. Davidson MTM, Yuen J, D'Souza DP, Radwan JS, Hammond JA, Batchelar DL. Optimization of high-dose-rate cervix brachytherapy applicator placement: The benefits of intraoperative ultrasound guidance. Brachytherapy 2008; 7: 248-53.

25. Brascho DJ. Use of pelvic pneumography in planning radiotherapy of endometrial carcinoma. Radiology 1970; 97: 113-20.

26. Scott WP. Tandem hysterography during intrauterine radium application. Am J Roentgenol Radium Ther Nucl Med 1975; 124: 636-7.

27. Pötter R, Dimopoulos J, Georg P, Lang S, Waldhäusl C, Wachter-Gerstner $\mathrm{N}$, et al. Clinical impact of MRI assisted dose volume adaptation and dose escalation in brachytherapy of locally advanced cervix cancer. Radiother Oncol 2007; 83: 148-55.

28. Haie-Meder C, Chargari C, Rey A, Dumas I, Morice P, Magné N. MRI-based low dose-rate brachytherapy experience in locally advanced cervical cancer patients initially treated by concomitant chemoradiotherapy. Radiother Oncol 2010; 96: 161-5.

29. De Brabandere M, Mousa AG, Nulens A, Swinnen A, Van Limbergen E. Potential of dose optimisation in MRI-based PDR brachytherapy of cervix carcinoma. Radiother Oncol 2008; 88: 217-26.

30. Lindegaard JC, Tanderup K, Nielsen SK, Haack S, Gelineck J. MRI-guided 3D optimization significantly pmproves DVH Parameters of pulsed-dose-rate brachytherapy in locally advanced cervical cancer. Int J Radiat Oncol Biol Phys 2008; 71: 756-64.

31. Pötter R, Georg P, Dimopoulos J, Grimm M, Berger D, Nesvacil N, et al. Clinical outcome of protocol based image (MRI) guided adaptive brachytherapy combined with 3D conformal radiotherapy with or without chemotherapy in patients with locally advanced cervical cancer. Radiother Oncol 2011; 100: 116-23.

32. Jürgenliemk-Schulz IM, Tersteeg RJ, Roesink JM, Bijmolt S, Nomden CN Moerland MA, et al. MRI-guided treatment-planning optimisation in intracavitary or combined intracavitary/interstitial PDR brachytherapy using tandem ovoid applicators in locally advanced cervical cancer. Radiother Oncol 2009; 93: 322-30.

33. Granai CO, Allee P, Doherty F, Madoc-Jones H, Curry SL. Ultrasound used for assessing the in situ position of intrauterine tandems. Gynecol Oncol 1984; 18: 334-8.

34. Granai CO, Allee P, Doherty F, Ball HG, Madoc-Jones H, Curry SL. Intraperative real-time ultrasonography during intrauterine tandem placement. Obstet Gynecol 1986; 67: 112-4.

35. Wong F, Bhimji S. The usefulness of ultrasonography in intracavitary radiotherapy using selectron applicators. Int J Radiat Oncol Biol Phys 1990; 19: $477-82$.

36. Tanaka S, Hishikawa Y, Kamikonya N, Miura T. Sonographic positioning of endouterine applicator. Radiat Med 1987; 5: 92-3.

37. Fleischer AC, Burnett LS, Murray MJ, Jones HW. Intraoperative guidance for intrauterine procedures with transrectal sonography. Radiology 1990; 176: 576-7.

38. Sharma DN, Rath GK, Thulkar S, Kumar S, Subramani V, Julka PK. Use of transrectal ultrasound for high dose rate interstitial brachytherapy for patients of carcinoma of uterine cervix. J Gynecol Oncol 2010; 21: 12-7. 


\title{
Long term follow-up report of cardiac toxicity in patients with multiple myeloma treated with tandem autologous hematopoietic stem cell transplantation
}

\author{
Mirta Kozelj¹, Samo Zver², Vesna Zadnik \\ ${ }^{1}$ Department of Cardiology, University Medical Centre Ljubljana, Ljubljana, Slovenia \\ ${ }^{2}$ Department of Haematology, University Medical Centre Ljubljana, Ljubljana, Slovenia \\ ${ }^{3}$ Institute of Oncology Ljubljana, Ljubljana, Slovenia
}

Radiol Oncol 2013; 47(2): 161-165.

Received 23 November 2012

Accepted 13 January 2013

Correspondence to: Prof. Mirta Koželj, MD, PhD., Department of Cardiology, University Medical Centre Ljubljana, Zaloska 7, SI-1525 Ljubljana, Slovenia. Phone: +386 1522 2844; Fax: +386 1522 2828; E-mail: mirta.kozelj@mf.uni-lj.si

Disclosure: No potential conflicts of interest were disclosed.

Background. Tandem autologous hematopoietic stem cell transplantation (ta-HSCT) is a standard treatment for multiple myeloma (MM). Patients receive a high-dose cyclophosphamide (CY), followed by two myeloablative cycles of melphalan (MEL). There are scarce data about long term cardiotoxicity.

Patients and methods. We studied 12 patients ( $62.25 \pm 8.55$ years) six years after the completion of MM treatment with ta-HCST. Late cardiotoxic effects were evaluated clinically and echocardiographically.

Results. None of the patients developed clinical signs of heart failure, all were in sinus rhythm and NT-pro BNP concentration was elevated $(778 \pm 902.76 \mathrm{pg} / \mathrm{mL})$. The left ventricular (LV) size remained normal. The LV ejection fraction did not decrease $(73.75 \pm 5.67 \%, 69.27 \pm 6.13 \%, p=N S)$. The LV diastolic function parameters $(E, A$, ratio $E / A$ and $A / a)$ did not change significantly. In tissue Doppler parameters we observed a nonsignificant decrease in $E_{m}(10.26 \pm 2.63$ $\mathrm{cm} / \mathrm{s}, 7.57 \pm 1.43 \mathrm{~cm} / \mathrm{s})$ and $S_{m}$ velocities $(8.7 \pm 0.87 \mathrm{~cm} / \mathrm{s}, 7.14 \pm 1.17 \mathrm{~cm} / \mathrm{s}, p=N S)$. The $E / E_{m}$ values were in an abnormal range $(8.66 \pm 1.05,10.55 \pm 2.03)$.

Conclusions. The treatment of MM with ta-HSCT, during which patients receive a high dose CY followed by two myeloablative cycles of MEL, causes mild, chronic, partially reversible and clinically silent cardiotoxic side-effects. However, ta-HSCT in patients with MM is a safe regarding cardiotoxic side effects, but, because of increasing life expectancy needs long term attention.

Key words: cardiotoxicity; cyclophosphamide; melphalan; echocardiography

\section{Introduction}

Autologous hematopoietic stem cell transplantation (HSCT) is one of the most efficient therapies for patients with multiple myeloma (MM). ${ }^{1}$ The intensification of the treatment with double autologous HSCT very likely results in increased survival rates for MM patients. ${ }^{2,3}$ In the era of novel agents, upfront tandem autologous hematopoietic stem cell transplantation (ta-HSCT) may not be as strong a treatment modality as some years ago. ${ }^{4}$ However, the procedure remains a powerful tool against the disease and if not performed upfront, it represents a reliable treatment option at the time of disease relapses.

Because our study started in the era before novel agents were available, included patients received vincristine, epirubicin, dexamethasone (VED) induction chemotherapy, followed by cyclophosphamide with granulocyte colonies stimulating factor (G-CSF) as part of the stem cell mobilization and collection regimen and first myeloablative cycle 
of melphalan $\left(200 \mathrm{mg} / \mathrm{m}^{2}\right)$. Patients who did not respond completely underwent a second ta-HSCT with the same dosage of melphalan within the next few months. They, thus, received a high dose cyclophosphamide and two myeloablative dosages of melphalan during a short period of time. Both chemotherapeutic agents may exert cardiotoxic side effects. ${ }^{5}$ Namely, neurohormonal activation of heart failure was observed during the treatment. Doppler echocardiography studies revealed the worsening of the left ventricular diastolic function and the occurrence of functional mitral regurgitation. We have found no reports on chronic cardiotoxic effects of cyclophosphamide and melphalan administered as a part of the treatment with taHSCT.

Cyclophosphamide doses greater than $1.5 \mathrm{~g} / \mathrm{m}^{2}$ may be associated with the acute cardiotoxic risk. ${ }^{6}$ Cardiac toxicity is manifested as acute-onset heart failure and/or myocarditis occurring within 1 to 10 days after the administration of the first dose of cyclophosphamide. Echocardiography revealed reversible reduction in the left ventricular ejection fraction. ${ }^{7}$

Cardiac toxicity of melphalan may manifests itself as paroxysmal artial fibrillation. ${ }^{8}$ The use of melphalan in combination with purine analogue fludarabine has been reported to produce acuteonset left heart failure, even in individuals with no history of prior heart disease. ${ }^{9}$ Prospective studies conducted to date have not confirmed long-term cardiotoxic effects of melphalan, yet cardiotoxic side effects of the drug cannot be ruled out completely.

In the former echocardiographic study of acute, subacute and early chronic cardiotoxic side effects of cyclophospamide and melphalan in the treatment MM with ta-HSCT, we confirmed a diastolic left ventricular dysfunction and the increase incidence of mitral regurgitation. ${ }^{5}$

Therefore, this study was undertaken to evaluate possible late cardiotoxic effects of the aforementioned drugs and prospectively investigate the cardiac function and indicators of heart failure in individuals six years after the treatment with taHSCT.

\section{Patients and Methods}

\section{Patients}

We studied 12 surviving patients (out of 30 patients initially treated) six years after successful autologous HSCT because of MM: 7 men and 5 women, aged 53 to $75(62.25 \pm 8.55)$. None of them had at presentation, nor later on in the course of the disease, the extramedullary form of MM involving the chest, cervical or thoracic spine that would require the treatment by irradiation of the thoracic cavity.

\section{Study design}

During the year 2005, thirty patients with MM underwent ta-HSCT. The trial started four weeks after the completion of the last out of three cycles of VAD chemotherapy, i.e. prior to the administration of cyclophosphamide at a dose of $4 \mathrm{~g} / \mathrm{m}^{2}$ as a part of the stem cell mobilization/collection regimen. Prior to the initiation of cyclophosphamide therapy, physical examination and baseline echocardiography (phase 0) were carried out. Two months after the cyclophosphamide therapy, the administration of the first myeloablative cycle of melphalan (200 $\mathrm{mg} / \mathrm{m}^{2}$ ) was administered. Four months after the administration of the first cycle of melphalan, the patients received the second myeloablative cycle of melphalan $\left(200 \mathrm{mg} / \mathrm{m}^{2}\right)$ as a part of the ta-HSCT. Three months after the patients had received the second cycle of melphalan, we made the final assessment of cardiac toxicity of cyclophosphamide and two successive myeloablative dosages of melphalan, based on the last physical examination and echocardiography (phase 1). In the year 2007, two years after the completion of autologous HSCT, the echocardiographic study was repeated (phase 2) and in the year 2011, six years after double autologous HSCT, we finished the trial with the fourth echocardiographic follow up (phase 3). At each step of the study we examined the patients regarding clinical signs and symptoms of heart failure and performed ECG. N-terminal prohormone of brain natriuretic peptide (NT-proBNP) serum concentrations were measured at the very end of the study.

\section{Echocardiography}

Each patient was evaluated at each phase with two-dimensional, conventional Doppler and tissue Doppler echocardiographic studies. All examinations at all times were carried out by the same examiner. We determined left ventricular end-diastolic (LV EDD), left ventricular systolic diameter (LV ESD), calculated the left ventricular ejection fraction (LV EF), and assessed the left atrial transversal and longitudinal diameters. The systolic function of the left ventricle was assessed also by 
the velocity time integral of the left outflow tract (VTI). The left ventricular diastolic function was assessed by measuring diastolic mitral inflow velocities (E and A-wave), pulmonary vein systolic (s-wave), diastolic (d-wave) and atrial reversal (awave) velocities. The E/A velocities index and A/a duration ratio were calculated as markers of the diastolic left ventricular function. In addition, tissue Doppler echocardiography of the mitral annulus was used for assessing the left ventricular systolic and diastolic function. Mitral annulus systolic $\left(\mathrm{S}_{\mathrm{m}}\right)$ and early mitral annulus diastolic tissue velocity $\left(E_{m}\right)$ were measured. The $E / E_{m}$ index was calculated as a marker of increased left atrial pressure. We used the mean value of three consecutive measurements for each of the parameters examined. Each examination included the evaluation for potential mitral regurgitation and pericardial effusion.

\section{Statistical analysis}

All analysed variables are distributed normally and are presented as the mean \pm standard deviation. The associations between NT-proBNP serum concentration and individual echocardiographic parameters were evaluated by calculating Pearson correlation coefficients. Repeated measures general linear model (GLM) with Bonferroni's multiple comparisons test was used to assess the possible significance of changes in each echocardiographic parameter in successive time points. A $p$ value of 0.05 or less was considered statistically significant. Statistical analyses were performed with the SPSS software package (version 13.0).

\section{Results}

\section{Patient data}

The study included 12 surviving patients with MM (7 men and 5 women) after successful double autologous HSCT. Eighteen out of 30 patients initially included in the study died because of MM progression during the period of six years. None of them complained of heart failure symptoms, nor did they manifest signs of it during the period mentioned.

All 12 enrolled surviving patients had normal baseline cardiovascular status. None of them developed clinically overt heart failure at any point in the study and electrocardiographic recordings disclosed no dynamic changes or rhythm abnormalities. In phase 3 , the average value of NT-proBNP was $778 \pm 902.76 \mathrm{pg} / \mathrm{mL}$ (range $124-2724 \mathrm{pg} / \mathrm{mL}$ ).

\section{Echocardiography}

The echocardiographic parameters are indicated in Table $1 .{ }^{10,11}$ The left ventricular and atrial size did not increase significantly in any phase of the study and remained within normal limits. Standard parameters for the left ventricular systolic function and LVEF did not decrease. The VTI decreased significantly between phases 0 and $2(p=0.04)$ but it recovered as late as six years after the treatment with double autologous HSCT. However, the values were within normal. The $S_{m}$ a very sensitive tissue Doppler parameter, mildly decreased throughout the study and finally the values dropped under the lowest reference values.

The standard parameters identifying the diastolic left ventricular function by mitral flow velocities (E, A, E/A, A duration) did not change significantly during the six years of observation. Nevertheless, the E/A ratio reached the criteria of the abnormal relaxation in phases 1 and 2 and was restored in the phase 3 . The duration of a wave in pulmonary vein flow decreased from phase 1 to 2 significantly $(p=$ $0.04)$. The ratio A/a improved from phase 1 to 2 significantly $(p=0.001)$. No statistical differences regarding the baseline values could be found in other pulmonary vein parameters. In tissue Doppler parameters we observed a mild progressive decrease of $E_{m}$ velocities without statistical significance. The $\mathrm{E} / \mathrm{E}_{\mathrm{m}}$ values were in an abnormal range for this age group from the phase $1 \mathrm{on}$. The $\mathrm{E} / \mathrm{E}_{\mathrm{m}}$ ratio showed the gradual increase during all phases with statistical significance $(p=0.01)$ and was in an abnormal range from phase $1 \mathrm{on}$. The only positive correlation was found between serum NT-proBNP concentration and $\mathrm{E} / \mathrm{E}_{\mathrm{m}}(\mathrm{p}=0.009)$.

The frequency of mitral regurgitation and pericardial effusion did not reach statistically significant difference between the baseline study and phase 2 or 3 .

There were no statistically significant differences between results of the baseline study (phase 0) and the results of the long term study in any of the observed parameters.

\section{Discussion}

Ta-HSCT is an efficient and frequently used treatment modality regimen for patients with MM. Patients treated with ta-HSCT develop immediate but transient, mostly reversible and clinically nonovert neurohormonal activation of heart failure in each phase of their treatment. Early post-treatment 
TABLE1. Echocardiographic characteristics

\begin{tabular}{|c|c|c|c|c|c|c|c|c|}
\hline & Phase 0 & Phase 1 & Phase 2 & Phase 3 & $\begin{array}{c}\text { Signif phase } \\
1: 2 p\end{array}$ & $\begin{array}{c}\text { Signif phase } \\
1: 3 p\end{array}$ & $\begin{array}{c}\text { Signf phase } \\
0: 2 p\end{array}$ & $\begin{array}{c}\text { Signif phase } \\
0: 3 p\end{array}$ \\
\hline LV EDD $(\mathrm{cm})$ & $4.69 \pm 0.4$ & $4.45 \pm 0.63$ & $4.63 \pm 0.38$ & $4.65 \pm 0.85$ & NS & NS & NS & NS \\
\hline LV ESD (cm) & $2.65 \pm 0.3$ & $2.7 \pm 0.35$ & $2.6 \pm 0.31$ & $2.87 \pm 0.56$ & NS & NS & NS & NS \\
\hline LA tr $(\mathrm{cm})$ & $4.06 \pm 0.54$ & $3.85 \pm 0.53$ & $3.93 \pm 0.45$ & $4.08 \pm 0.92$ & NS & NS & NS & NS \\
\hline LA long (cm) & $4.4 \pm 0.43$ & $4.42 \pm 0.47$ & $4.34 \pm 0.75$ & $4.67 \pm 0.79$ & NS & NS & NS & NS \\
\hline LV EF (\%) & $73.75 \pm 5.67$ & $68.66 \pm 9.3$ & $75 \pm 4.41$ & $69.27 \pm 6.13$ & NS & NS & NS & NS \\
\hline$E(m / s)$ & $0.86 \pm 0.16$ & $0.76 \pm 0.16$ & $0.74 \pm 0.18$ & $0.79 \pm 0.13$ & NS & NS & 0.02 & NS \\
\hline$A(m / s)$ & $0.88 \pm 0.16$ & $0.89 \pm 0.16$ & $0.82 \pm 0.21$ & $0.82 \pm 0.2$ & 0.05 & NS & NS & NS \\
\hline$E / A$ & $1.03 \pm 0.34$ & $0.88 \pm 0.26$ & $0.97 \pm 0.43$ & $1.05 \pm 0.42$ & NS & NS & NS & NS \\
\hline$A / a$ & $1.3 \pm 0.28$ & $0.98 \pm 0.22$ & $1.34 \pm 0.3$ & $1.28 \pm 0.25$ & 0.001 & NS & NS & NS \\
\hline $\mathrm{S}_{\mathrm{m}}(\mathrm{cm} / \mathrm{s})$ & $8.7 \pm 0.87$ & $8.1 \pm 2.68$ & $7.77 \pm 0.97$ & $7.14 \pm 1.17$ & NS & NS & NS & NS \\
\hline $\mathrm{E}_{\mathrm{m}}(\mathrm{cm} / \mathrm{s})$ & $10.26 \pm 2.63$ & $7.78 \pm 1.44$ & $7.68 \pm 1.58$ & $7.57 \pm 1.43$ & NS & NS & NS & NS \\
\hline$E / E_{m}$ & $8.66 \pm 1.05$ & $10.43 \pm 1.69$ & $9.69 \pm 1.79$ & $10.55 \pm 2.03$ & NS & 0.01 & NS & NS \\
\hline $\mathrm{PE}(\mathrm{N})$ & 1 & 2 & 2 & 3 & NS & NS & NS & NS \\
\hline$M R(N)$ & 4 & 6 & 1 & 3 & NS & NS & NS & NS \\
\hline
\end{tabular}

LV EDD = left ventricular end diastolic volume; LV ESD = left ventricular end systolic volume; LA tr = transverse diameter of the left atrium; LA long = longitudinal diameter of the left atrium: $\mathrm{LVEF}=$ left ventricular ejection fraction: $\mathrm{E}=$ mitral early diastolic flow velocity: $\mathrm{A}=$ mitral $\mathrm{A}$-wave velocity: $\mathrm{E} / \mathrm{A}=\mathrm{ratio}$ in mitral diastolic flow; $\mathrm{A} / \mathrm{a}=\mathrm{ratio}$ between the left atrium; $L \mathrm{EF}=$ left ventricular ejection fraction; $E=$ mitral early diastolic flow velocity; $A=$ mitral $A$-wave velocity; $E / A=$ ratio in mitral diastolic flow; $A / a=$ ratio between the
duration of mitral $A$-wave and duration of $a$-wave in pulmonary venous flow; $s / d=$ ratio between systolic and diastolic pulmonary venous flow; $S_{m}=$ mitral annulus systolic tissue velocity; $\mathrm{E}_{m}=$ early mitral annulus diastolic tissue velocity; $\mathrm{E}_{\mathrm{m}}=\mathrm{E}_{\mathrm{m}}=$ ratio between mitral early diastolic flow velocity ( $\mathrm{E}$ ) and early mitral annulus diastolic tissue velocity ( $\mathrm{E}_{\mathrm{m}}$ ); Phase $0=$ at baseline; Phase 1 = three months after the patients had received the second dosage of melphalan; Phase $2=$ two years after successful double autologous HSCT; Phase 3 = six years after double autologous HSCT; Normal values ${ }^{10,11}$; PE = pericardial effusion; MR = mitral regurgitation

abnormalities including diastolic dysfunction with abnormal left ventricular relaxation, elevated left atrial pressure and functional mitral regurgitation are detected echocardiographicaly. ${ }^{12}$ Very little is known about eventual chronic cardiac toxicity of this treatment regimen, which includes cyclophosphamide and a high dose melphalan as part of stem cell mobilisation and collection, followed by two autologous HSCT. There are no long term trials on cardiotoxicity in this treatment modality.

In this long term study, we followed up 12 survivors out of 30 treated MM patients with ta-HSCT. The rest, 18 out of 30 patients initially included, died of MM progression and did not suffer from symptoms of heart failure at any time. In the 12 currently alive MM patients, the clinical examination did not reveal any signs of heart failure.

ECG did not show any rhythm disturbances. They were all in sinus rhythm. The left atrial and ventricular size and left ventricular ejection fraction did not show any significant changes in a period of six years after the completion of therapy and all parameters were in the range of normal values. This result is as expected, because these are rather nonsensitive parameters of cardiotoxicity. The VTI, Doppler echocardiographic marker of systolic left ventricular function, was in the range of normal values as well, although it showed a mild decrease for the period of the follow-up but recovered to baseline values within six years. The tissue Doppler parameters are more sensitive and we noticed a gradual decrease in $S_{\mathrm{m}}$ the values did not reach normal range even after the completion of the therapy. The $S_{m}$ decrease was not statistically significant, probably because of the small number of patients. Therefore, we can conclude that there are some indices for a mild systolic dysfunction at long term follow-up. We suggest that stress echocardiography may be helpful in solving this question.

Diastolic dysfunction parameters are much more reliable and sensitive markers of cardiotoxicity than parameters of the left ventricular systolic function, as has been proven for acute cardiotoxicity in many Doppler echocardiographic studies. ${ }^{12-14}$ The E/A ratio reached the criteria of the abnormal 
relaxation after the treatment and we noticed its recovery to the normal ratio not earlier than at the six-year follow-up. The changes in duration of the transmitral A wave and the pulmonary a wave also showed a pattern significant for the elevated left atrial pressure, especially the $\mathrm{A} / \mathrm{a}$ ratio. Despite that, the A/a ratio normalized as early as two years after the completion of the therapy. At the end of the follow-up there were no significant differences in Doppler echocardiographic diastolic function markers between the baseline study and the final study. So the conventional echocardiographic methods exclude late complications of cardiotoxic therapy with a high dose cyclophosphamide and melfalan in the context of ta-HSCT. In recent years, tissue Doppler echocardiography has been used with the aim to identify acute and chronic subclinical alterations of the left ventricular function in patients treated by antracyclines, while we have very little experience with tissue Doppler echocardiography in patients treated by other chemotherapeutic agents. ${ }^{15}$ In our group of patients, we noticed abnormal $\mathrm{E}_{\mathrm{m}}$ velocities from the completion of therapy on and further on the values reached neither normal nor baseline levels at the end of the study. We believe that the decrease of $\mathrm{E}_{\mathrm{m}}$ velocities did not reach statistical significance because of the small number of patients. The $\mathrm{E} / \mathrm{E}_{\mathrm{m}}$ ratio, a marker of elevated filling pressure of the left ventricle, was increasing throughout the follow-up and at the end of the study exceeded the value immediately after the completion of the therapy; it was in the abnormal range. ${ }^{11}$ This result is suggestive of the elevated filling pressures late after the therapy. Positive correlation in the serum concentration of NT-proBNP and $E / E_{m}$ supports this fact. The highest values of NT-proBNP were present in two patients with the lowest $\mathrm{E} / \mathrm{E}_{\mathrm{m}}$.

\section{Conclusions}

We conclude that the treatment of patients with MM with ta-HSCT, in which the patients receive a high dose cyclophosphamide and two myeloablative dosages of melphalan, causes mild, chronic, partially reversible, but clinically silent cardiotoxic side effects. The most reliable parameters were NTproBNP and tissue Doppler parameters of diastolic function of the left ventricle. To determine this with certainty, we need studies with a greater number of patients and stress echocardiographic studies. Long term studies with a longer period of observational time would not improve our knowledge of long term side effects because of the influence of age on Doppler and especially on tissue Doppler parameters.

However, our study shows that ta-HSCT in patients with MM represents a safe treatment modality regarding cardiotoxic side effects, but because life expectancy in these patients is increasing, needs a long term follow up.

\section{References}

1. Attal M, Harousseau JL, Stoppa AM, Sotto JJ, Fuzibet JG, Rossi JF, et al. A prospective, randomized trial of autologous bone marrow transplantation and chemotherapy in multiple myeloma. N Engl J Med 1996; 335: 91-7.

2. Barlogie B, Jagannath S, Vesole DH, Naucke S, Cheson B, Mattox S, et al. Superiority of tandem autologous transplantation over standard therapy for previously untreated multiple myeloma. Blood 1997; 89: 789-93.

3. Attal M, Harousseau JL, Facon T, Guilhot F, Doyen C, Fuzibet JG, et al. Single versus double autologous stem-cell transplantation for multiple myeloma. N Engl J Med 2003; 349: 2495-502.

4. Palumbo A, Anderson K. Medical progress. Multiple myeloma. N Engl J Med 2011; 364: 1046-60.

5. Zver S, Zadnik V, Cernelc P, Koželj M. Cardiac toxicity of high-dose cyclophosphamide and melphalan in patients with multiple myeloma treated with tandem autologous hematopoietic stem cell transplantation. Int $J$ Haematology 2008; 88: 227-36.

6. Braverman AC, Antin JH, Plappert MT. Cyclophosphamide cardiotoxicity in bone marrow transplantation: a prospective evaluation of new dosing regimens. J Clin Oncol 1991; 9: 15-33.

7. McDonagh TA, Robb SD, Murdoch DR Morton JJ, Ford I, Morrison CE, et al. Biochemical detection of left-ventricular systolic dysfunction. Lancet 1998; 351: 9-13.

8. Tsuruda T, Boerrigter G, Huntley BK, Noser JA, Cataliotti A, CostelloBoerrigter LC, et al. BNP is produced in cardiac fibroblasts and induces matrix metalloproteinases. Circ Res 2002; 91: 1127-34.

9. Maisel AS, Krishnaswamy P, Nowak RM, McCord J, Hollander JE, Duc P, et al Rapid measurement of B-type natriuretic peptide in the emergency diagnosis of heart failure. N Engl J Med 2002; 347: 61-7.

10. Lang RM, Bierig M, Devereux RB, Flachskampf FA, Foster E, Pellikka PA et al. Recommendations for chamber quantification: a report from the American Society of Echocardiography's Guidelines and Standards Committee and the Chamber Quantification Writing Group, developed in conjunction with the European Association of Echocardiography, a branch of the European Society of Cardiology. J Am Soc Echocardiogr 2005; 18: 1440-63.

11. Chahal NS, Lim TK, Jain P, Chambers JC, Kooner JS, Senior R, et al. Normative reference values for the tissue Doppler imaging parameters of left ventricular function: a population-based study. Eur J Echocardiogr 2010; 11: 51-6.

12. Giannuzzi $\mathrm{P}$, Temporelli $\mathrm{PL}$, Bosimini $\mathrm{E}$, Silva $\mathrm{P}$, Imparato $\mathrm{A}$, Corrà $\mathrm{U}$, et al. Independent and incremental prognostic value of Doppler-derived mitral deceleration time of early filling in both symptomatic and asymptomatic patients with left ventricular dysfunction. J Am Coll Cardiol 1996; 28: 83-90.

13. Galderisi M, Marra F, Esposito R, Lomoriello VS, Pardo M, de Divitiis O. Cancer therapy and cardiotoxicity: the need of serial Doppler echocardiography. Cardiovascular Ultrasound 2007; 5: 4.

14. Benvenuto GM, Ometto R, Fontanelli A, Fortunato A, Ruffini PA, Fosser V, et al. Chemotherapy related cardiotoxicity: new diagnostic and preventive strategies. Ital Heart J 2003; 4: 655-67.

15. Tassan-Mangina, S, Codorean D, Metivier M, Costa B, Himberlin C, Jouannaud C, et al. Tissue Doppler imaging and conventional echocardiography after anthracycline treatment in adults: early and late alterations of left ventricular function during prospective study. Eur J Echocardiography 2006; 7: 141-6. 


\title{
A review of the treatment options for skin rash induced by EGFR-targeted therapies: Evidence from randomized clinical trials and a meta- analysis
}

\author{
Janja Ocvirk ${ }^{1}$, Steffen Heeger², Philip McCloud ${ }^{3}$, Ralf-Dieter Hofheinz ${ }^{4}$ \\ ${ }^{1}$ Institute of Oncology, Ljubljana, Slovenia \\ ${ }^{2}$ Merck KGaA, Darmstadt, Germany \\ ${ }^{3}$ McCloud Consulting Group, Sydney, Australia \\ ${ }^{4}$ Universitätsmedizin Mannheim, University of Heidelberg, Germany
}

Radiol Oncol 2013; 47(2): 166-175.

Received: 15 November, 2012

Accepted: 15 December, 2012

Correspondence to: Assist. Prof. Janja Ocvirk, M.D., Ph.D., Institute of Oncology Ljubljana, Zaloška 2, SI-1000 Ljubljana, Slovenia. Phone: +386 15879 220; Fax: +386 15879 305; E-mail: jocvirk@onko-i.si.

Disclosure: No potential conflicts of interest were disclosed.

Background. Agents targeting the epidermal growth factor receptor (EGFR) are amongst the most extensively used of the targeted agents in the therapy of some of the most common solid tumors. Although they avoid many of the classic side effects associated with cytotoxic chemotherapy, they are associated with unpleasant cutaneous toxicities which can affect treatment compliance and impinge on patient quality of life. To date, despite a plethora of consensus recommendations, expert opinions and reviews, there is a paucity of evidence-based guidance for the management of the skin rash that occurs in the treatment of patients receiving EGFR-targeted therapies.

Methods. A literature search was conducted as a first step towards investigating not only an evidence-based approach to the management of skin rash, but also with a view to designing future randomized trials.

Results. The literature search identified seven randomized trials and a meta-analysis was conducted using the data from four of these trials involving oral antibiotics. The meta-analysis of the data from these four trials suggests that prophylactic antibiotics might reduce the relative risk of severe rash associated with EGFR-targeted agents by $42-77 \%$. Vitamin $\mathrm{K}$ cream was also identified as having a potential role in the management EGFR-targeted agent induced rash.

Conclusions. This review and meta-analysis clearly identify the need for further randomized studies of the role of oral antibiotics in this setting. The results of the ongoing randomized trials of the topical application of vitamin $\mathrm{K}$ cream plus or minus doxycycline and employing prophylactic versus reactive strategies are eagerly awaited.

Key words: acne-like skin rash; cetuximab; antibiotics; erlotinib; gefitinib; panitumumab; vitamin K

\section{Introduction}

Recognition of the importance of the epidermal growth factor receptor (EGFR [HER1]) in tumorigenesis and tumor progression ${ }^{1-5}$ led to the development of EGFR-targeted therapies, including the monoclonal antibodies (mAbs) cetuximab and panitumumab, and the EGFR tyrosine kinase inhibitors (TKIs) gefitinib, erlotinib and lapatinib, for use in the therapy of a range of solid tumors including those of the colon and rectum, head and neck, lung, pancreas and breast. ${ }^{6}$ Although, the toxicity profiles of EGFR-targeted therapies, across the different malignancies, largely exclude many of the severe side-effects observed with cytotoxic agents (e.g. hematological side-effects and hair loss), they are associated with the development of cutaneous toxicities. ${ }^{7-12}$ The earliest and most com- 
TABLE 1. Summary of the different forms of cutaneous toxicities induced by epidermal growth factor receptor (EGFR)-targeted agents $8,12,14$

\begin{tabular}{|c|c|}
\hline Site & Description of cutaneous toxicity \\
\hline Skin & $\begin{array}{l}\text { Acneiform skin rash } \\
\text { Erythema } \\
\text { Eczema } \\
\text { Photosensitivity } \\
\text { Fissures, rhagades } \\
\text { Xerotic skin and pruritus } \\
\text { Hyperpigmentation and teleangiectasia }\end{array}$ \\
\hline Nails & $\begin{array}{l}\text { Paronychia } \\
\text { Fissures }\end{array}$ \\
\hline Hair & $\begin{array}{l}\text { Trichomegaly (growth of eyelashes) } \\
\text { Hypertrichosis } \\
\text { Alopecia }\end{array}$ \\
\hline Eyes & $\begin{array}{l}\text { Conjunctivitis } \\
\text { Blepharitis } \\
\text { Sicca syndrome, increased lacrimation }\end{array}$ \\
\hline
\end{tabular}

mon of these, for both classes of EGFR-targeted agents, is a papulopustular skin rash ${ }^{13}$, although the variety of terms used to describe skin rash has made direct comparisons of its incidence between trials and agents complicated..$^{10}$ Other well-documented cutaneous toxicities associated with these agents include xerosis, pruritus, and specific hair and nail changes (Table 1). ${ }^{8,12,14}$ An overview of the incidence of skin toxicities with different EGFRtargeted agents, according to their licensed indications is presented in Table 2.

Generally, the cutaneous toxicities associated with these targeted agents are classified as mild to moderate, but if left untreated they can potentially affect both patient quality of life (QoL) $)^{15-17}$ and treatment compliance. ${ }^{18,19}$ They can also predispose the skin to bacterial, fungal, or viral infections. Also, given the association between the manifestation of cutaneous toxicities and the clinical efficacy of EGFR-targeted treatment approaches, ${ }^{10,20-24}$ it is becoming increasingly clear that therapeutic and preventive strategies need to be, and should be, adopted in the management of such toxicities to facilitate treatment continuation whilst maintaining maximal patient tolerability and the avoidance of treatment delays and interruptions.

This narrative review will focus on the reactive and prophylactic approaches, for the improved management of the cutaneous toxicities, specifically rash, induced by EGFR-targeted agents, investigated in recent randomized clinical trials. As a meta-analysis is a systematic approach to identification and abstraction of critical information from different randomized ${ }^{25}$, controlled trials, we also made the meta-analysis of the data obtained from four of these recent randomized trials investigating the use of antibiotics.

\section{Methods}

A search of Medline and of the abstracts on oncology meeting databases provided the literature sources on which this review is based. This search identified four manuscripts, reporting the data from four individual randomized trials on the skin toxicities caused by EGFR targeted agents that could be combined in a meta-analysis of skin toxicity. The primary results from the meta-analysis of these four trials will also be reported.

\section{Results}

\section{EGFR-targeted agent induced skin toxicity \\ Grading and treatment strategies}

To date, the National Cancer Institute Common Terminology Criteria for Adverse Events (NCICTCAE) version 3.0 grading scale, published in 2006, is the scale that has been used most often for grading the skin toxicities induced by EGFRtargeted therapies. However, this scale is not optimal for grading the skin toxicities, and in particular the papulopustular acneiform rash, induced by this class of agent. As a consequence an updated version of this grading scale ${ }^{26}$, and other grading systems have been proposed..$^{27,28}$ In addition, the lack of a standardized approach to both the grading and management of skin reactions induced by EGFR-targeted therapies has been identified. ${ }^{29}$

It is against this background that at least five sets of consensus recommendations have been published ${ }^{8,9,29-31}$, together with expert opinions and recommendations for the treatment of the skin toxicities associated with EGFR-targeted therapies in general ${ }^{12,19,26,33,34}$, and the radiation dermatitis seen in patients with locally advanced squamous cell carcinoma of the head and neck (SCCHN) receiving cetuximab concomitantly with radiotherapy ${ }^{35}$, in particular. There have also been several reviews. ${ }^{10,11,36-38}$ Most have detailed strategies utilizing the topical application of emollient and antibiotic and steroid creams, administration of systemic steroids and antibiotics, avoidance of sun exposure, and the use of high-protection factor sun creams, for the management of skin rash. To date, there has been at least one randomized trial of the prophylactic use of sunscreen ${ }^{39,40}$, five randomized 
TABLE 2. Incidences of skin toxicity with EGFR-targeted agents in different licensed indications ${ }^{8,63-67}$

\begin{tabular}{|c|c|c|c|}
\hline \multirow[t]{2}{*}{ Drug } & \multirow[t]{2}{*}{ Indication } & \multicolumn{2}{|c|}{ Skin toxicities, \% } \\
\hline & & All grades & Grade 3/4 \\
\hline \multicolumn{4}{|c|}{ EGFR-specific monoclonal antibodies } \\
\hline Panitumumab & $\begin{array}{l}\text { Metastatic KRAS wild-type CRC } \\
\text { Monotherapy (after failure of fluoropyrimidine-, oxaliplatin- and irinotecan regimen) } \\
\text { Combination with oxaliplatin-containing chemotherapy } \\
\text { Combination with irinotecan-containing chemotherapy }\end{array}$ & $\begin{array}{l}90 \\
22^{a} \\
96 \\
N R\end{array}$ & $\begin{array}{l}16 \\
10 \\
36 \\
37\end{array}$ \\
\hline \multicolumn{4}{|c|}{ EGFR small molecule tyrosine kinase inhibitors } \\
\hline
\end{tabular}

aRash only

EGFR = epidermal growth factor receptor; $\mathrm{mCRC}=$ metastatic colorectal cancer; $\mathrm{NR}=$ not reported; $\mathrm{NSCLC}=$ non-small cell lung cancer; $\mathrm{SCCHN}=$ squamous cell carcinoma of the head and neck

trials of oral antibiotics (Table 3$)^{16,41-44}$, and one of the prophylactic use of topical pimecrolimus, an immunomodulator ${ }^{45}$, developed for the treatment of inflammatory skin disease. These randomized studies were conducted with a view to providing a much needed evidence base for the refinement of the treatment approaches used in the management of the cutaneous toxicities experienced by patients receiving EGFR-targeted therapies. They are discussed in detail below according to individual category.

\section{Randomized trials}

\section{Antibiotics and skin rash}

Standard tetracycline is commonly used for the treatment of acne, and the similarity between acne and EGFR-targeted agent-induced skin rash suggested a possible role for tetracycline in the treatment or prevention of the skin rash that occurs following treatment with these agents. ${ }^{41}$ It was also thought that the anti-inflammatory effects of tetracycline might provide effective rash palliation. Thus, following on from several case/small studies $^{46-48}$, there have been five randomized trials of the use of standard tetracycline and tetracyclineclass (minocycline and doxycycline) antibiotics in the treatment of skin rash in patients receiving EGFR-targeted therapies.

\section{Tetracycline}

The first of these was a randomized, placebo-controlled, phase III trial (N03CB), conducted in the US by the North Central Cancer Treatment Group (NCCTG). In this trial, 61 cancer patients (31 lung, 16 CRC and 15 other malignancies), starting treatment with an EGFR-targeted agent (cetuximab or gefitinib), were randomized to receive either tetracycline (500 mg/bid for 28 days) or placebo. ${ }^{41}$ The primary objective of the study was to compare the incidence of rash in patients treated with prophylactic tetracycline versus placebo. Patients were evaluated at the end of weeks 4 and 8, for performance status, adverse events and rash according to NCI-CTCAE version 3.0. In addition, the skin-specific, health-related QoL questionnaire Skindex-16 ${ }^{49}$ was used to measure the effects on patient QoL.

The incidence of rash was found to be comparable across the two arms. Sixteen (70\%) patients treated with tetracycline and 22 (76\%) patients treated with placebo developed physician-reported rash $(p=0.61)$, during the first 4 weeks. During the subsequent 4 weeks, when patients were no longer receiving either tetracycline or placebo, physician-reported rash was recorded for 13 tetracycline-treated patients (87\%) and 16 placebo-treated patients $(84 \%)(\mathrm{p}=0.84)$. There were however, some indications that tetracycline might have had 
an effect on rash severity. By week 4, physicianreported grade 2 rash was recorded for 4 (17\%) tetracycline-treated patients compared with 16 $(55 \%)$ placebo-treated patients $(\mathrm{p}=0.04)$ (Table 3$)$. By week 8, physician-reported rash was reported in $27 \%$ and $47 \%$ of tetracycline- and placebotreated patients $(p=0.5)$, respectively. Results for patient-reported rash also suggested slightly better outcomes for those patients receiving tetracycline. In addition, patients treated with tetracycline also reported less itching, burning, stinging and skin irritation than patients treated with placebo.

The results of this study suggested therefore that the prophylactic use of oral tetracycline might be beneficial ${ }^{41}$, despite the fact that the primary endpoint of the trial was not achieved. As a consequence the authors concluded that because of the preliminary benefits observed and the general acceptance of tetracycline for the treatment of skin rashes, there should be no objection to its prescription to reduce the severity of the rash associated with EGFR-targeted agents. Unfortunately, however, the confirmatory NCCTG supplementary randomized N03CB trial conducted in 65 patients (33 in the tetracycline arm and 32 in the placebo arm) of whom $>50 \%$ had metastatic colorectal cancer ( $\mathrm{mCRC}$ ) and $>60 \%$ received cetuximab, failed to demonstrate any benefit conferred by tetracycline in terms of either the incidence or severity of rash in patients receiving therapy with EGFR-targeted agents. ${ }^{42}$

\section{Tetracycline-class antibiotics Minocycline}

However, an earlier randomized trial of the broadspectrum tetracycline antibiotic minocycline, in 48 patients with $\mathrm{mCRC}$, showed that patients receiving prophylactic oral minocycline, on the same day as initiation of therapy with the EGFR-targeted $\mathrm{mAb}$ cetuximab, had a lower mean facial lesion count than those receiving placebo (Table 3$){ }^{43}$

In this trial, the time course of the development of cetuximab-induced rash was comparable with that reported for previous studies, ${ }^{12,13}$ with rash developing rapidly following the start of therapy, peaking weeks 2-4, and becoming less severe as treatment continued. Clinical assessments included questionnaires and skin examinations at the end of weeks 1, 2, 4 and 8. In addition, photographic images of the face were reviewed by two independent dermatologists who were blind to the treatment arms. Total facial lesion counts were significantly lower in patients receiving minocycline weeks 1-4. Also, a lower proportion of patients in the minocycline arm reported moderate-severe itch than in the placebo arm $(20 \%$ versus $50 \%$, p = $0.05)$. A review of the severity of facial rash showed 4 patients (20\%) with severe rash in the minocycline arm compared with 8 patients (42\%) in the placebo arm $(\mathrm{p}=0.13)$. The difference in the number of facial lesions and subjectively assessed itch had reduced by week 8 . Prophylactic minocycline was therefore considered to be effective at reducing the severity of rash occurring during the first 4 weeks of treatment with cetuximab, but could not be recommended for use beyond 8 weeks.

\section{Doxycycline}

The randomized phase II trial 'Skin Toxicity Evaluation Protocol with Panitumumab' (the STEPP study) was the first to investigate the preemptive (prophylactic) versus reactive use of skin treatment strategies which included the use of moisturizers, sunscreen, topical steroids and oral doxycyline (100 mg/bid), a semi-synthetic tetracycline. Patients with mCRC $(n=95)$ being treated with the EGFR-targeted agent panitumumab and randomly assigned 1:1 to one or other treatment strategy, showed grade $\geq 2$ skin toxicity to be reduced from $62 \%$ in the reactive patient group to $29 \%$ in the prophylactic patient group during the 6 -week skin treatment period (odds ratio 0.3, 95\% confidence interval [CI] 0.1-0.6). ${ }^{16}$ In addition, grade 2 and 3 skin toxicities of interest were reported in $23 \%$ and $40 \%$ of patients, and $6 \%$ and $21 \%$ of patients, for the prophylactic and reactive treatment arms, respectively. Furthermore, patients in the prophylactic treatment group reported improved QoL, between weeks 2 and 3 in particular, which was the median time to development of the first grade $\geq 2$ skin toxicity in the reactive treatment group..$^{50}$ The results of this study clearly support the prophylactic use of antibiotics in the management of EGFR-targeted agent-induced skin toxicity.

In another large randomized trial (CYTAR) investigating the prophylactic use of doxycycline versus placebo in 147 NSCLC patients being treated with the TKI erlotinib, those patients receiving doxycycline showed a marked reduction in the severity of Grade $\geq 2$ erlotinib-induced folliculitis from $82 \%$ to $39 \%$ (Table 3 ). ${ }^{44}$ Patients were randomly assigned to erlotinib with or without doxycycline (100 mg/day). Serial photographs were taken for blind review. The primary objective of the study was to assess the efficacy of doxycycline in reducing the incidence of erlotinib-induced folliculitis during the first 4 months of treatment and the 
TABLE 3. Oral antibiotics in the treatment of skin rash

\begin{tabular}{|c|c|c|c|c|c|c|c|c|}
\hline Reference & $\begin{array}{l}\text { Patients } \\
\text { (n) }\end{array}$ & $\begin{array}{l}\text { Patient } \\
\text { characteristics }\end{array}$ & Antibiotic & $\begin{array}{l}\text { Duration of } \\
\text { skin treatment }\end{array}$ & Endpoint & $\begin{array}{l}\text { Assessment } \\
\text { tools }\end{array}$ & $\begin{array}{l}\text { Skin toxicity } \\
\text { Results } \\
\text { Placebo vs } \\
\text { intervention }\end{array}$ & $\begin{array}{l}\text { Skin-related } \\
\text { quality of life }\end{array}$ \\
\hline $\begin{array}{l}\text { NCCTG NO3CB } \\
\text {-Jatoi et al., } \\
2008^{39}\end{array}$ & 61 & $\begin{array}{l}\text { Lung/ } \\
\text { gastrointestinal/ } \\
\text { other patients } \\
\text { treated with } \\
\text { gefitinib, } \\
\text { cetuximab, } \\
\text { erlotinib/other } \\
\text { investigational } \\
\text { agent }\end{array}$ & Tetracycline & $\begin{array}{l}500 \mathrm{mg} \text { bid } \\
4 \text { weeks }\end{array}$ & $\begin{array}{l}\text { Incidence of } \\
\text { Grade }>2 \text { skin } \\
\text { rash, QoL }\end{array}$ & $\begin{array}{l}\mathrm{NCl}-\mathrm{CTCAE} \\
\text { version } 3.0, \\
\text { Skindex } 16\end{array}$ & $\begin{array}{l}76 \% \text { vs } 70 \% \\
\text { developed a } \\
\text { rash. } \\
\text { Grade } 2 \\
55 \% \text { vs } 17 \% \text { at } \\
\text { week } 4\end{array}$ & $\begin{array}{l}\text { Less skin } \\
\text { irritation, } \\
\text { burning or } \\
\text { stinging } \\
\text { (Skindex-16) } \\
\text { in tetracycline } \\
\text { arm }\end{array}$ \\
\hline $\begin{array}{l}\text { Supplementary } \\
\text { NCCTG NO3CB- } \\
\text { Jatoi et al., } 2011^{40}\end{array}$ & 65 & As above & Tetracycline & $\begin{array}{l}500 \mathrm{mg} \text { bid } \\
4 \text { weeks }\end{array}$ & $\begin{array}{l}\text { Incidence of } \\
\text { Grade }>2 \text { skin } \\
\text { rash, } \\
\text { QoL }\end{array}$ & $\begin{array}{l}\mathrm{NCI}-\mathrm{CTCAE} \\
\text { version } 3.0 \text {, } \\
\text { Skindex } 16 \text { and } \\
\text { LASA. }\end{array}$ & $\begin{array}{l}\text { Grade } 2 \\
\text { identical }\end{array}$ & $\begin{array}{l}\text { Identical } \\
\text { (Skindex-16) }\end{array}$ \\
\hline $\begin{array}{l}\text { Scope et al., } \\
2007^{41}\end{array}$ & 48 & $\begin{array}{l}\text { mCRC patients } \\
\text { treated with } \\
\text { cetuximab }\end{array}$ & Minocycline & $\begin{array}{l}100 \mathrm{mg} / \mathrm{d} \\
8 \text { weeks }\end{array}$ & $\begin{array}{l}\text { Total facial } \\
\text { lesion counts; }\end{array}$ & $\begin{array}{l}\text { Photography } \\
\text { and } \\
\text { patient- } \\
\text { assessed } \\
\text { rash severity } \\
\text { and other } \\
\text { cutaneous } \\
\text { changes }\end{array}$ & $\begin{array}{l}\text { Lower facial } \\
\text { lesion count } \\
\text { during } \\
\text { weeks } 1-4 \text { in } \\
\text { minocyline } \\
\text { arm ( } p=0.005 \text { ), } \\
\text { Less severe } \\
\text { facial rash } \\
\text { (42\% vs } 20 \%\end{array}$ & $\begin{array}{l}\text { Less severe } \\
\text { itching in } \\
\text { minocycline } \\
\text { arm, } \\
50 \% \text { vs } 20 \% \\
(p=0.05)\end{array}$ \\
\hline $\begin{array}{l}\text { Lacouture et al., } \\
2010^{15}\end{array}$ & 95 & $\begin{array}{l}\text { Previously treated } \\
\text { mCRC patients } \\
\text { treated with } \\
\text { panitumumab- } \\
\text { containing } \\
\text { therapy; }\end{array}$ & Doxycycline & $\begin{array}{l}100 \mathrm{mg} \text { bid } \\
6 \text { weeks } \\
+ \\
\text { skin moisturizer } \\
\text { and sunscreen }\end{array}$ & $\begin{array}{l}\text { Prophylactic } \\
\text { vs reactive. } \\
\text { incidence } \\
\text { of protocol } \\
\text { specified } \\
\text { Grade }>2 \text { skin } \\
\text { rash, } \\
\text { QoL }\end{array}$ & $\begin{array}{l}\mathrm{NCl}-\mathrm{CTCAE} \\
\text { version 3.0, } \\
\text { DLQI }\end{array}$ & $\begin{array}{l}\text { Grade } 2 \\
\text { reduced from } \\
62 \% \text { in the } \\
\text { reactive group } \\
\text { to } 29 \% \text { in the } \\
\text { prophylactic } \\
\text { group }\end{array}$ & $\begin{array}{l}\text { Better (DLQI) } \\
\text { in prophylactic } \\
\text { group; } \\
\text { change from } \\
\text { baseline } \\
\text { score less for } \\
\text { prophylactic } \\
\text { than reactive } \\
\text { group }\end{array}$ \\
\hline $\begin{array}{l}\text { Deplanque et al., } \\
2010^{42}\end{array}$ & 147 & $\begin{array}{l}\text { Non-small cell lung } \\
\text { cancer patients } \\
\text { treated with } \\
\text { erlotinib; all }\end{array}$ & Doxycycline & $\begin{array}{l}100 \mathrm{mg} / \mathrm{d} \\
4 \mathrm{months}\end{array}$ & $\begin{array}{l}\text { Incidence } \\
\text { of erlotinib } \\
\text { folliculitis }\end{array}$ & $\begin{array}{l}\mathrm{NCl}-\mathrm{CTCAE} \\
\text { version } 3.0\end{array}$ & $\begin{array}{l}\text { Incidence } 82 \% \\
\text { vs } 68 \% \text {, } \\
\text { Grade } \geq 2 \\
\text { reduced from } \\
82 \% \text { to } 39 \% \text {, } \\
\text { also significant } \\
\text { decrease } \\
\text { in other } \\
\text { cutaneous } \\
\text { AEs. }\end{array}$ & na \\
\hline
\end{tabular}

$\mathrm{AEs}=$ adverse events; $\mathrm{DLQI}=$ Dermatological Life Quality Index; na = not available; NCI-CTCAE = National Cancer Institute Common Terminology Criteria for Adverse Events; $\mathrm{mCRC}=$ metastatic colorectal cancer

secondary objective to assess the impact of doxycycline on rash severity. The incidence of folliculitis was $71 \%$ in patients pretreated with doxycycline and $82 \%$ in those who were not pretreated $(\mathrm{p}=$ $0.117)$. This difference approached significance $(p$ $=0.055)$ when those patients who did not actually take doxycycline were excluded from the analysis of the doxycyline arm. Doxycyline was shown to significantly reduce the severity of all erlotinibinduced folliculitis ( $p=<0.001$ ), and the severity of other treatment-induced cutaneous AEs. The trial results also indicated that QoL was significantly less impaired in the doxycycline arm compared with the placebo arm at day $14(\mathrm{p}=0.04)$.

Thus, overall, a significant reduction in the severity of skin rash was noticed in three ${ }^{16,41,44}$ out of the four randomized studies ${ }^{16,41,2,44}$ using NCI-CTCAE version 3.0 criteria for the evaluation. The greatest effect of the use of oral antibiotics probably occurs during the first 6-8 weeks of treatment, and the best data at this time would appear to derive from the two studies using doxycycline. ${ }^{16,44}$ Certainly, the CYTAR trial is the largest randomized trial to date to investigate the prophylactic use of a drug against EGFR-targeted agent-induced skin rash, and while the overall incidence of skin rash was not reduced, patients receiving doxycycline showed a significant reduction in the severity of their erlotinib-induced folliculitis and significantly less impairment of their QoL. Furthermore, the use of doxycycline did not affect the clinical efficacy outcomes of the EGFR-targeted therapies, namely panitumumab or erlotinib, in either trial. The data, therefore, would appear to support the prophylactic use of doxycycline for patients receiving EGFR-targeted therapy. Significantly also, with the exception of one standard tetracycline trial ${ }^{42}$, all the other antibiotic trials (Table 3) indicate that the skin-related QoL is better in the group receiving prophylactic antibiotics regardless of the assessment tool used. These obser- 
vations are in part consistent with the data from a small meta-analysis which was conducted on four of the trials as outlined below.

\section{Meta-analysis of antibiotics in the treatment of skin rash}

From a review of the literature it was determined that the data from four of the above studies investigating the use of antibiotics could be combined ${ }^{41-44}$ (Table 3) in meta-analyses of rash incidence and rash severity. The randomized STEPP study ${ }^{16}$, with panitumumab, was excluded from the analysis because it had no placebo arm.

For each study the relative risk was used to measure and test the differences in rash incidence between treatments ${ }^{51}$, with Cochran's Q statistic used to test the heterogeneity of the relative risks between the studies. Fixed and random effects estimates were calculated and compared..$^{52}$ Two metaanalyses of the incidence of EGFR-targeted agent associated rash were performed, one including and one excluding the study of minocycline in patients with $\mathrm{mCRC}$ treated with cetuximab..$^{43}$ This was because the relative risk for this study ${ }^{43}$ was based on the presence of rash rather than the incidence of moderate/severe rash. Both analyses of rash incidence, i.e.: without and with the minocyline study (Figures 1 and 2), showed non-significant heterogeneity between the relative risks according to Cochran's $Q$ statistic. The fixed and random effect analyses were identical/nearly identical and the combined relative risks were not significantly different from 1.0. Neither meta-analysis provided strong evidence that the use of an antibiotic reduced the incidence of rash associated with treatment with EGFR-targeted agents.

Conversely, the meta-analysis of severe rash (Figure 3) showed significant heterogeneity between relative risks according to Cochran's $Q$ statistic, and the fixed and random effect analyses were not similar. The estimated relative risk that appears to have caused the significant heterogeneity is the negative tetracycline study. ${ }^{42}$ The estimated relative risks indicate that the risk of severe rash was reduced by $42-47 \%$ with the use of an antibiotic to control rash associated with the use of EGFR targeted agents (Figure 3). However, based on these data it is possible that tetracycline is not effective and that additional confirmatory studies are needed to validate the potential roles of doxycycline and minocycline. Thus, it is probably too early to claim doxycycline to be a 'pseudo-stand-

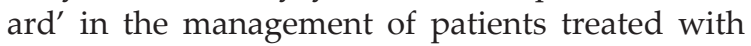
EGFR-targeted therapies.

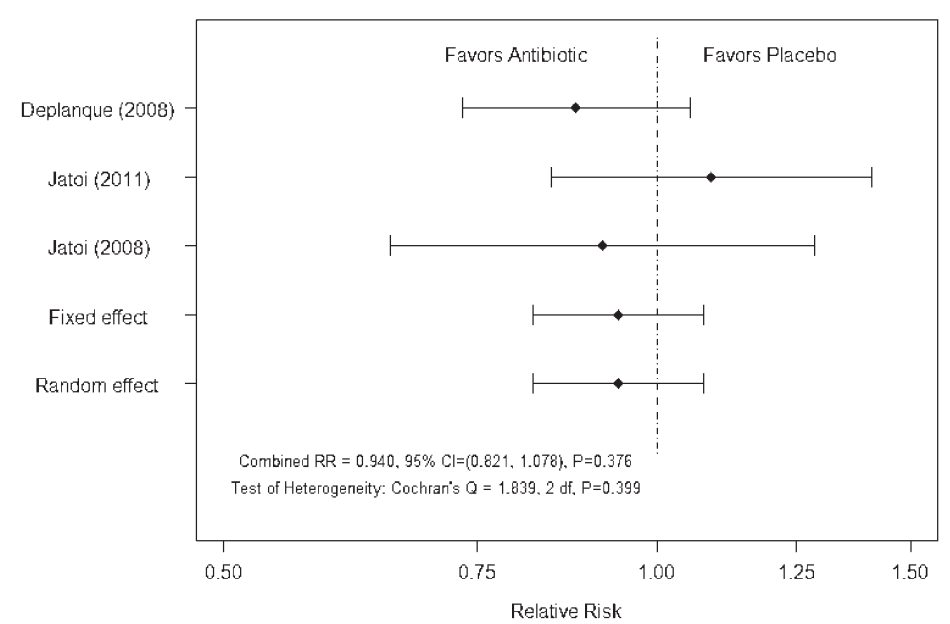

FIGURE 1. Meta-analysis of rash incidence excluding study of Scope et al. ${ }^{43}$

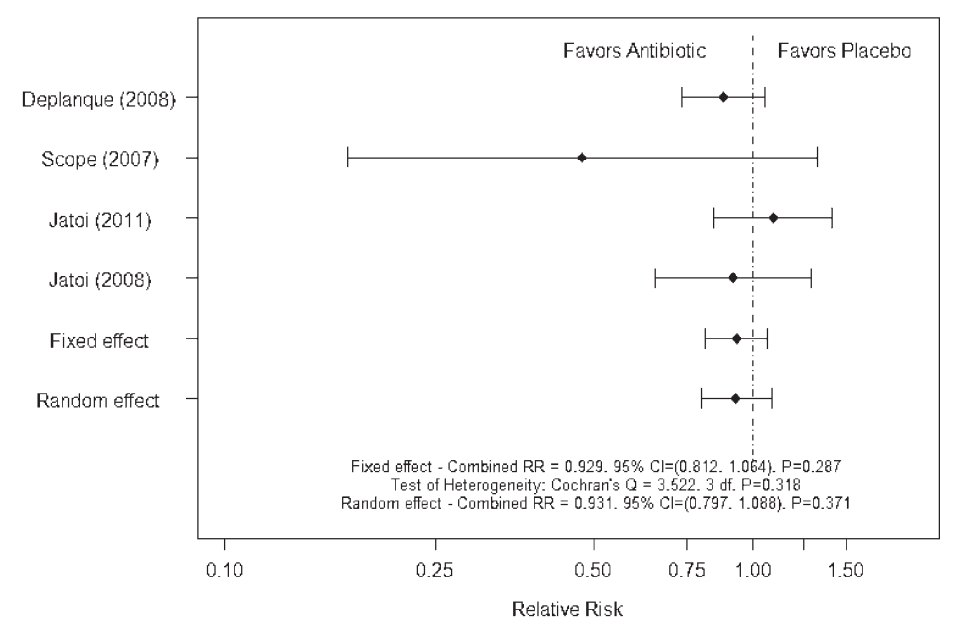

FIGURE 2. Meta-analysis of rash incidence including study of Scope et al. ${ }^{43}$

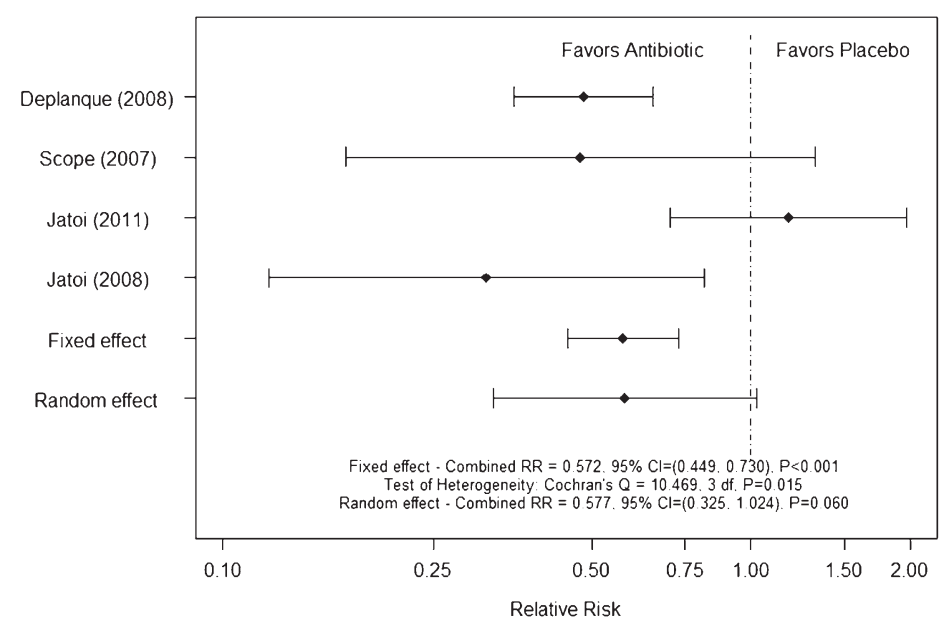

FIGURE 3. Meta-analysis of rash severity 
TABLE 4. Ongoing trials of Vitamin $\mathrm{K}$ in the management of skin toxicities induced by EGFR-targeted agents

\begin{tabular}{|c|c|c|c|c|c|}
\hline Trial & $\begin{array}{l}\text { Planned } \\
\text { patients (n) }\end{array}$ & Patient characteristics & Design & Primary endpoint & Secondary endpoint(s) \\
\hline $\begin{array}{l}\text { EVITA } \\
\text { NCTO1345526 }\end{array}$ & 124 & $\begin{array}{l}\text { mCRC } \\
\text { FOLFIRI + cetuximab }\end{array}$ & $\begin{array}{l}\text { Vitamin } \mathrm{K} 1 \text { cream } \\
+ \\
\text { Oral doxycycline } \\
\text { versus } \\
\text { Placebo } \\
+ \\
\text { Oral doxycycline }\end{array}$ & $\begin{array}{l}\text { Occurrence of acne-like } \\
\text { skin rash grade >2 } \\
\text { National Cancer Institute } \\
\text { Common Terminology } \\
\text { Criteria for Adverse } \\
\text { Events version } 4.0\end{array}$ & $\begin{array}{l}\text { Response } \\
\text { Quality of life } \\
\text { WoMo score } 25\end{array}$ \\
\hline NCT01094444 & 36 & $\begin{array}{l}\text { Chemotherapy plus } \\
\text { cetuximab in } \\
\text { mCRC or squamous cell } \\
\text { carcinoma of the head } \\
\text { and neck patients }\end{array}$ & $\begin{array}{l}\text { Prophylactic } \\
\text { versus } \\
\text { Reactive application of } \\
\text { vitamin K3 lotion }\end{array}$ & $\begin{array}{l}\text { Reduction of cutaneous } \\
\text { side effects }\end{array}$ & $\begin{array}{l}\text { Investigation of possible } \\
\text { side-effects of lotion }\end{array}$ \\
\hline
\end{tabular}

EGFR = epidermal growth factor receptor; FOLFIRI = irinotecan, 5-fluorouracil, leucovorin; $\mathrm{mCRC}=$ metastatic colorectal cancer

\section{Topical treatment approaches}

Topical treatments that might provide an alternative treatment approach to oral antibiotics also need to be considered, as topical treatment approaches are used extensively in this setting.

\section{Sunscreen and prevention of skin rash}

Some patients receiving EGFR-targeted agents have been reported to show widespread erythema, infiltration and pustules in sun-exposed areas. ${ }^{19,53,54}$ One randomized study, N05C4, conducted in the US by NCCTG, has investigated the prophylactic use of SPF60 sunscreen in cancer patients receiving an EGFR-targeted agent as part of their therapy. ${ }^{39}$

Prior to randomization, patients in the study were stratified according to first-line cancer therapy, type of EGFR-targeted agent prescribed or anticipated (TKI versus $\mathrm{mAb}$ ), and the use of any concurrent medication that might be associated with increased sensitivity to sun exposure. ${ }^{40}$ Overall, 110 rash-free patients (39 lung, 45 CRC and 26 other malignancy) were randomized to receive an application of SPF60 sunscreen twice daily for 4 weeks versus placebo. Patients were monitored for rash and QoL using the skin-specific questionnaire Skindex-16 ${ }^{49}$ during the 4 weeks of topical application and for the 4 weeks following cessation of the treatment intervention. ${ }^{40}$ The primary objective of the study was to compare the incidence of rash in sunscreen- and placebo-treated patients. During the 4 weeks of intervention 78\% and 80\% of patients developed physician-reported skin rash in the sunscreen and placebo arms, respectively. Furthermore, no significant difference in the incidence of rash was reported for the subsequent 4 weeks. However, the incidence of higher-grade skin rash (grade 2 or $>50 \%$ of body surface area) was lower, $33 \%$ versus $52 \%(p=0.06)$, in the sunscreen arm of the study at 4 weeks. ${ }^{40}$

Although in this study, prophylactic treatment with sunscreen failed to reduce the overall incidence of skin rash, the fact that the incidence of higher-grade skin rash was markedly reduced in the sunscreen arm of the study led the study investigators to conclude that the prophylactic use of sunscreen should be continued in patients receiving EGFR-targeted therapy.

\section{Tazarotene}

A parallel study of topical tazarotene, a retinoic acid receptor specific retinoid, in the same patients as those investigated in the randomized minocyline trial, showed tazarotene to be ineffective with considerable patient attrition in the tazarotene portion of the study. ${ }^{43}$

\section{Pimecrolimus}

The authors of the randomized minocycline trial also conducted a randomized trial of the topical application of the immunomodulator pimecrolimus to one side of the face for patients with $\mathrm{mCRC}$ receiving cetuximab therapy ${ }^{45}$, and although the treated sides of the face had a greater decrease in lesion counts, this did not translate into a clinically meaningful benefit for patients. 


\section{Vitamin K}

Vitamin K1 (phylloquinone) is naturally occurring. Vitamin K has been used for the treatment of blood vessel disorders of the skin, cosmetic skin treatment and skin treatment following laser treatment. It is thought to prevent disruption of the balance between proliferation and differentiation, thinning of the epidermis, immune reaction and inflammatory reactions leading to folliculitis. Topical vitamin K3 (Menadione) prevents erlotinib- and cetuximab-induced EGFR inhibition in the skin. ${ }^{55-57}$

To date, there have been four observational studies investigating the topical application of $0.1 \%$ vitamin $\mathrm{K} 1$ cream, three reactive ${ }^{58-60}$ and one prophylactic. ${ }^{61}$ In all three reactive studies good control of EGFR-targeted therapy-induced rash was observed. In one study, in $79 \mathrm{mCRC}$ patients premedicated with an $\mathrm{H} 1$ antagonist and corticosteroids, receiving weekly cetuximab plus chemotherapy ${ }^{59}$, the topical application of a cream containing urea and $0.1 \%$ vitamin $\mathrm{K} 1$ twice daily on the appearance of acne-like rash (NCI-CTCAE v. 3.0), reduced the severity of the rash. The median time to improvement was 1.2 weeks and the median time to downstaging of the rash by $\geq 1$ grade was 2.3 weeks. ${ }^{59}$ In another study, patients receiving cetuximab/panitumumab for the treatment of mCRC were treated with vitamin $\mathrm{K} 1$ cream at the first onset of grade $\geq 2$ skin rash. The median duration of vitamin $\mathrm{K} 1$ cream treatment was 24 weeks (range, 6-28). Thirteen patients (39.4\%) also received oral tetracycline therapy. A decrease in skin rash to grade $0-1$ was observed in $12(36.4 \%)$ patients, and $13(39.4 \%)$ patients showed unchanged grade 2 skin toxicity. Overall, good skin rash symptom control was obtained in $69.2 \%$ of patients. ${ }^{60}$

In the prophylactic study ${ }^{61}$, the use of vitamin $\mathrm{K} 1$ cream $(0.1 \%$ bid) applied to the face and chest was very effective at reducing the severity of cetuximab-induced skin toxicity (NCI-CTCAE 3.0) in 48 patients with $\mathrm{mCRC}$ receiving cetuximab in combination with chemotherapy, first-line. Application of vitamin $\mathrm{K} 1$ cream delayed the development of acne-like rash which peaked in the third week, and reduced the need for topical and systemic antibiotic treatment. All skin toxicities were grade $1 / 2$ and no cetuximab dose reductions or treatment delays were required.

Thus, overall, topical vitamin K1 treatment has demonstrated significant clinical efficacy in the absence of any toxicity, with the limited evidence favoring a prophylactic treatment approach. Currently, there are no randomized trial data for the use of vitamin $\mathrm{K}$ therapy in the management of EGFR-targeted therapy-induced skin toxicities, although three randomized trials are ongoing (Table 4), one of which (EVITA), a double-blind, controlled, phase II study is evaluating the efficacy and safety of the prophylactic use of doxycycline +/- vitamin K cream in mCRC patients treated with chemotherapy plus cetuximab, first-line.

\section{Discussion}

EGFR-agent-induced skin toxicities can be effectively treated at all stages and grade and are generally considered to be completely reversible except for telangiectasias. Their management is important and needs to be considered at as early a stage as possible as a prerequisite to maintaining patient QoL while continuing EGFR-targeted therapy. Evidence from two of the studies described above, with different treatment approaches, namely the STEPP study $^{16}$ for interventions containing oral antibiotics and the prophylactic study of vitamin $\mathrm{K} 1 \mathrm{cream}^{61}$, suggest that prophylactic approaches should be the strategy of choice, but the agents of choice remain to be fully established as well as their schedule of administration. Certainly the data for doxycycline and vitamin $\mathrm{K}$ cream provide considerable cause for optimism in standardizing the management particularly of the early skin toxicities associated with therapy involving EGFRtargeted agents. The most recent guidelines by MASCC for the prevention of EGFR-targeted agent induced skin toxicities, recommend the preventive/prophylactic management of skin rash whenever possible, based on the observations from the randomized sunscreen trial ${ }^{40}$, and three of the randomized antibiotic trials. ${ }^{16,41,43}$ The recommended preventive therapy for the development of rash from the MASCC group would be the topical application of a steroid cream with moisturizer and sunscreen twice daily and systemic minocycline or doxycycline. ${ }^{62}$ Other studies that might be considered with a view to establishing and/or further defining the role of such treatment approaches in the management of EGFR-targeted agent-induced rash might include prophylactic doxycycline at different doses and randomized studies of:

Prophylactic doxycycline versus prophylactic vitamin K therapy

Prophylactic doxycycline plus prophylactic vitamin $\mathrm{K}$ therapy versus prophylactic vitamin $\mathrm{K}$ therapy versus prophylactic doxycycline therapy. 


\section{Acknowledgements}

The authors wish to thank Dr. Anne Kinsella Ph.D. (Cancer Communications \& Consultancy Ltd, Knutsford, UK, funded by Merck KGaA, Darmstadt, Germany) for medical writing services.

\section{References}

1. Bianco R, Gelardi T, Damiano V, Ciardiello F, Tortora G. Rational bases for the development of EGFR inhibitors for cancer treatment. Int J Biochem Cell Biol 2007; 39: 1416-31

2. Downward J, Yarden Y, Mayes E, Scrace G, Totty N, Stockwell P, et al. Close similarity of epidermal growth factor receptor and v-erb-B oncogene protein sequences. Nature 1984; 307: 521-7.

3. Nicholson RI, Gee JM, Harper ME. EGFR and cancer prognosis. Eur J Cancer 2001; 37(Suppl 4): S9-15.

4. Shelton JG, Steelman LS, Abrams SL, Bertrand FE, Franklin RA, McMahon $\mathrm{M}$, et al. The epidermal growth factor receptor gene family as a target for therapeutic intervention in numerous cancers: what's genetics got to do with it? Expert Opin Ther Targets 2005; 9: 1009-30.

5. Yarden Y, Sliwkowski MX. Untangling the ErbB signalling network. Nat Rev Mol Cell Biol 2001; 2: 127-37.

6. Rebersek M, Boc M, Cerkovnik P, Benedik J, Hlebanja Z, Volk N, Novakovic $S$, Ocvirk J. Efficacy of first-line systemic treatment in correlation with BRAF V600E and different KRAS mutations in metastatic colorectal cancer - a single institution retrospective analysis. Radiol Oncol 2011; 45: 285-91.

7. Jia Y, Lacouture ME, Su X, Wu S. Risk of skin rash associated with erlotinib in cancer patients: a meta-analysis. J Support Oncol 2009; 7: 211-7.

8. Lynch TJ Jr, Kim ES, Eaby B, Garey J, West DP, Lacouture ME. Epiderma growth factor receptor inhibitor-associated cutaneous toxicities: an evolving paradigm in clinical management. Oncologist 2007; 12: 610-21.

9. Melosky B, Burkes R, Rayson D, Alcindor T, Shear N, Lacouture M Management of skin rash during EGFR-targeted monoclonal antibody treatment for gastrointestinal malignancies: Canadian recommendations. Curr Oncol 2009; 16: 16-26.

10. Perez-Soler R, Delord JP, Halpern A, Kelly K, Krueger J, Sureda BM, et al. HER1/EGFR inhibitor-associated rash: future directions for management and investigation outcomes from the HER1/EGFR inhibitor rash management forum. Oncologist 2005; 10: 345-56.

11. Saif MW, Kim R. Incidence and management of cutaneous toxicities associated with cetuximab. Expert Opin Drug Saf 2007; 6: 175-82.

12. Segaert S, Van Cutsem E. Clinical signs, pathophysiology and management of skin toxicity during therapy with epidermal growth factor receptor inhibitors. Ann Oncol 2005; 16: 1425-33.

13. Agero AL, Dusza SW, Benvenuto-Andrade C, Busam KJ, Myskowski P Halpern AC. Dermatologic side effects associated with the epidermal growth factor receptor inhibitors. J Am Acad Dermatol 2006; 55: 657-70.

14. Ehmann LM, Ruzicka T, Wollenberg A. Cutaneous side-effects of EGFR inhibitors and their management. Skin Therapy Lett 2011; 16: 1-3.

15. Eilers RE Jr, Gandhi M, Patel JD, Mulcahy MF, Agulnik M, Hensing T, et al. Dermatologic infections in cancer patients treated with epidermal growth factor receptor inhibitor therapy. J Natl Cancer Inst 2010; 102: 47-53.

16. Lacouture ME, Mitchell EP, Piperdi B, Pillai MV, Shearer H, lannotti N, et al. Skin toxicity evaluation protocol with panitumumab (STEPP), a phase II, open-label, randomized trial evaluating the impact of a pre-emptive skin treatment regimen on skin toxicities and quality of life in patients with metastatic colorectal cancer. J Clin Oncol 2010; 28: 1351-7.

17. Oslo A, Mateurs C, Soria JC, Smith A. Cutaneous side effects in patients on long-term treatment with epidermal growth factor receptor inhibitors. $\mathrm{Br} J$ Dermatol 2009; 161: 515-21.

18. Lacouture M. The growing importance of skin toxicity in EGFR inhibito therapy. Oncology (Williston Park) 2009; 23: 194-6.
19. Robert C, Soria JC, Spatz A, Le Cesne A, Malka D, Pautier P, et al. Cutaneous side-effects of kinase inhibitors and blocking antibodies. Lancet Oncol 2005; 6: 491-500.

20. Peeters M, Price T, Van Laethem JL. Anti-epidermal growth factor receptor monotherapy in the treatment of metastatic colorectal cancer: where are we today? Oncologist 2009; 14: 29-39.

21. Perez-Soler R, Saltz L. Cutaneous adverse effects with HER1/EGFR-targeted agents: is there a silver lining? J Clin Oncol 2005; 23: 5235-46.

22. Racca P, Fanchini L, Caliendo V, Ritorto G, Evangelista W, Volpatto R, et al. Efficacy and skin toxicity management with cetuximab in metastatic colorectal cancer: outcomes from an oncologic/dermatologic cooperation. Clin Colorectal Cancer 2008; 7: 48-54.

23. Saltz LB, Meropol NJ, Loehrer PJ Sr, Needle MN, Kopit J, Mayer RJ. Phase II trial of cetuximab in patients with refractory colorectal cancer that expresses the epidermal growth factor receptor. J Clin Oncol 2004; 22: 1201-8.

24. Wacker B, Nagrani T, Weinberg J, Witt K, Clark G, Cagnoni PJ. Correlation between development of rash and efficacy in patients treated with the epidermal growth factor receptor tyrosine kinase inhibitor erlotinib in two large phase III studies. Clin Cancer Res 2007; 13: 3913-21.

25. Kovac V, Smrdel U. Meta-analyses of clinical trials in patients with non-small cell lung cancer. Minireview. Neoplasma 2004; 51: 334"1-40.

26. Potthoff K, Hofheinz R, Hassel JC, Volkenandt M, Lordick F, Hartmann JT, et al. Interdisciplinary management of EGFR-inhibitor-induced skin reactions: a German expert opinion. Ann Oncol 2011; 22: 524-35.

27. Wollenberg A, Moosmann $\mathrm{N}$, Klein $\mathrm{E}$, Katzer $\mathrm{K}$. A tool for scoring of acneiform skin eruptions induced by EGF receptor inhibition. Exp Dermatol 2008; 17: 790-2.

28. Lacouture ME, Maitland ML, Segaert S, Setser A, Baran R, Fox LP, et al. A proposed EGFR inhibitor dermatologic adverse event-specific grading scale from the MASCC skin toxicity study group. Support Care Cancer 2010; 18: 509-22.

29. Hassel JC, Kripp M, Al-Batran S, Hofheinz RD. Treatment of epidermal growth factor receptor antagonist-induced skin rash: results of a survey among German oncologists. Onkologie 2010; 33: 94-8.

30. Bernier J, Bonner J, Vermorken JB, Bensadoun RJ, Dummer R, Giralt J, et al. Consensus guidelines for the management of radiation dermatitis and coexisting acne-like rash in patients receiving radiotherapy plus EGFR inhibitors for the treatment of squamous cell carcinoma of the head and neck. Ann Oncol 2008; 19: 142-9.

31. Lacouture ME. Insights into the pathophysiology and management of dermatologic toxicities to EGFR-targeted therapies in colorectal cancer. Cancer Nurs 2007; 30(4 Suppl 1): S17-26

32. Segaert S, Van Cutsem E. Clinical management of EGFRI dermatologic toxicities: the European perspective. Oncology (Williston Park) 2007; 21: 22-6.

33. Galimont-Collen AF, Vos LE, Lavrijsen AP, Ouwerkerk J, Gelderblom H Classification and management of skin, hair, nail and mucosal side-effects of epidermal growth factor receptor (EGFR) inhibitors. Eur J Cancer 2007; 43: $845-51$.

34. Segaert S, Tabernero J, Chosidow O, Dirschka T, Elsner J, Mancini L, et al. The management of skin reactions in cancer patients receiving epidermal growth factor receptor targeted therapies. J Dtsch Dermatol Ges 2005; 3: 599-606.

35. Bernier J, Russi EG, Homey B, Merlano MC, Mesia R, Peyrade F, et al. Management of radiation dermatitis in patients receiving cetuximab and radiotherapy for locally advanced squamous cell carcinoma of the head and neck: proposals for a revised grading system and consensus management guidelines. Ann Oncol 2011; 22: 2191-200.

36. Hu JC, Sadeghi P, Pinter-Brown LC, Yashar S, Chiu MW. Cutaneous side effects of epidermal growth factor receptor inhibitors: clinical presentation, pathogenesis, and management. J Am Acad Dermatol 2007; 56: 317-26.

37. Tsimboukis S, Merikas I, Karapanagiotou EM, Saif MW, Syrigos KN. Erlotinibinduced skin rash in patients with non-small-cell lung cancer: pathogenesis, clinical significance, and management. Clin Lung Cancer 2009; 10: 106-11.

38. Wu PA, Balagula Y, Lacouture ME, Anadkat MJ. Prophylaxis and treatment of dermatologic adverse events from epidermal growth factor receptor inhibitors. Curr Opin Oncol 2011; 23: 343-51. 
39. Jatoi A. Sunshine and rash: testing the role of sunscreen to prevent epidermal growth factor receptor inhibitor-induced rash. Support Cancer Ther 2007; 4: 198-202.

40. Jatoi A, Thrower A, Sloan JA, Flynn PJ, Wentworth-Hartung NL, Dakhil SR, et al. Does sunscreen prevent epidermal growth factor receptor (EGFR) inhibitor-induced rash? Results of a placebo-controlled trial from the North Central Cancer Treatment Group (N05C4). Oncologist 2010; 15: 1016-22.

41. Jatoi A, Rowland K, Sloan JA, Gross HM, Fishkin PA, Kahanic SP, et al. Tetracycline to prevent epidermal growth factor receptor inhibitor-induced skin rashes: results of a placebo-controlled trial from the North Central Cancer Treatment Group (NO3CB). Cancer 2008;113: 847-53.

42. Jatoi A, Dakhil SR, Sloan JA, Kugler JW, Rowland KM Jr, Schaefer PL, et al. Prophylactic tetracycline does not diminish the severity of epiderma growth factor receptor (EGFR) inhibitor-induced rash: results from the North Central Cancer Treatment Group (Supplementary NO3CB). Support Care Cancer 2011; 19: 1601-7.

43. Scope A, Agero AL, Dusza SW, Myskowski PL, Lieb JA, Saltz L, et al. Randomized double-blind trial of prophylactic oral minocycline and topical tazarotene for cetuximab-associated acne-like eruption. J Clin Oncol 2007; 25: 5390-6.

44. Deplanque G, Chavaillon J, Vergnenegre A, Falchero L. CYTAR: A randomized clinical trial evaluating the preventive effect of doxycycline on erlotinibinduced folliculitis in non-small cell lung cancer patients [Abstract]. J Clin Oncol 2010; 28: Abstract 9019.

45. Scope A, Lieb JA, Dusza SW, Phelan DL, Myskowski PL, Saltz L, et al. A prospective randomized trial of topical pimecrolimus for cetuximab-associated acnelike eruption. J Am Acad Dermatol 2009; 61: 614-20.

46. Fernandez-Guarino M, Perez Garcia B, Aldanondo Fernandez de la Mora I, Garcia-Millan C, Garrido Lopez P, Jaen Olasolo P. [Treatment of acneiform rash by epidermal growth factor inhibitors with oral tetracyclines] [Spanish]. Actas Dermosifiliogr 2006; 97: 503-8.

47. Micantonio T, Fargnoli MC, Ricevuto E, Ficorella C, Marchetti P, Peris K Efficacy of treatment with tetracyclines to prevent acneiform eruption secondary to cetuximab therapy. Arch Dermatol 2005; 141: 1173-4.

48. Molinari E, De Quatrebarbes J, Andre T, Aractingi S. Cetuximab-induced acne. Dermatology 2005; 211: 330-3.

49. Chren MM, Lasek RJ, Sahay AP, Sands LP. Measurement properties of Skindex-16: a brief quality-of-life measure for patients with skin diseases. $J$ Cutan Med Surg 2001; 5: 105-10.

50. Mitchell E, Lacouture $M$, Shearer $\mathrm{H}$, lannotti N. Final STEPP results of prophylactic versus reactive skin toxicity (ST) treatment (tx) for panitumumab (pmab)-related ST in patients (pts) with metastatic colorectal cancer (mCRC) [Abstract]. J Clin Oncol 2009; 27: Abstract CRA4027.

51. Hedges LV, Olkin I. Statistical methods for meta-analysis. Orlando: Academic Press; 1985.

52. Laird NM, Ware JH. Random-effects models for longitudinal data. Biometrics 1982; 38: 963-74.

53. Luu M, Lai SE, Patel J, Guitart J, Lacouture ME. Photosensitive rash due to the epidermal growth factor receptor inhibitor erlotinib. Photodermatol Photoimmunol Photomed 2007; 23: 42-5.

54. Segaert S, Simonart T. The epidermal vitamin D system and innate immunity: some more light shed on this unique photoendocrine system? Dermatology 2008; 217: 7-11.

55. Li T, Perez-Soler R. Skin toxicities associated with epidermal growth factor receptor inhibitors. Target Oncol 2009; 4: 107-19.

56. Perez-Soler R, Zou Y, Li T, Ling Y. Steroids and immunosuppressive agents potentiate the cytotoxicity of the EGFR inhibitor erlotinib (E) in human skin keratinocytes whereas Vit K3 exerts a protective effect: implications for the management of the skin rash [Abstract]. J Clin Oncol 2007; 25: Abstract 9124

57. Perez-Soler R, Zou Y, Li T, Tornos C, Ling Y. Topical vitamin K3 (Vit K3, Menadione) prevents erlotinib and cetuximab-induced EGFR inhibition in the skin [Abstract]. J Clin Oncol 2006; 23: Abstract 3036.

58. Ocvirk J, Rebersek M. Management of cutaneous side effects of cetuximab therapy with vitamin K1 creme. Radiol Oncol 2008; 42: 215-24.

59. Ocvirk J, Rebersek M. Topical application of vitamin K1 cream for cetuximabrelated skin toxicities [Abstract]. Ann Oncol 2009; 20: Abstract PD-0021.
60. Pinto C, Barone C, Martoni A, Di Tullio P, Di Fabio F, Cassano A. Vitamin K1 cream in the management of skin rash during anti-EGFR monocloncal antibody (mAb) treatment in patients with metastatic cancer: first analysis of an observational Italian study [Abstract]. J Clin Oncol 2011; 29: Abstract 594.

61. Ocvirk J, Rebersek M, Boc M, Mesti T, Ebert M. Prophylactic use of K1 cream for reducing skin toxicity during cetuximab treatment in patients with metastatic colorectal cancer (mCRC) [Abstract]. J Clin Oncol 2010; 28: Abstract e14011.

62. Lacouture ME, Anadkat MJ, Bensadoun RJ, Bryce J, Chan A, Epstein JB, et al. Clinical practice guidelines for the prevention and treatment of EGFR inhibitor-associated dermatologic toxicities. Support Care Cancer 2011; 19: 1079-95.

63. Potthoff K, Hassel JC, Wollenberg A. Therapie und prophylaxe EGFRinhibitor-induzierter hautreaktionen. Arzneimitteltherapie 2010; 28: 191-8.

64. Peeters M, Price TJ, Cervantes A, Sobrero AF, Ducreux M, Hotko Y, et al. Randomized phase III study of panitumumab with fluorouracil, leucovorin, and irinotecan (FOLFIRI) compared with FOLFIRI alone as second-line treatment in patients with metastatic colorectal cancer. J Clin Oncol 2010; 28: 4706-13.

65. Douillard JY, Siena S, Cassidy J, Tabernero J, Burkes R, Barugel M, et al Randomized, phase III trial of panitumumab with infusional fluorouracil, leucovorin, and oxaliplatin (FOLFOX4) versus FOLFOX4 alone as first-line treatment in patients with previously untreated metastatic colorectal cancer: the PRIME study. J Clin Oncol 2010; 28: 4697-705.

66. Bokemeyer C, Bondarenko I, Makhson A, Hartmann JT, Aparicio J, de Braud $\mathrm{F}$, et al. Fluorouracil, leucovorin, and oxaliplatin with and without cetuximab in the first-line treatment of metastatic colorectal cancer. J Clin Oncol 2009; 27: 663-71.

67. Van Cutsem E, Kohne CH, Lang I, Folprecht G, Nowacki MP, Cascinu S, et al. Cetuximab plus irinotecan, fluorouracil, and leucovorin as first-line treatment for metastatic colorectal cancer: updated analysis of overall survival according to tumor KRAS and BRAF mutation status. J Clin Oncol 2011; 29: 2011-9. 


\title{
Does initial 45Gy of pelvic intensity-modulated radiotherapy reduce late complications in patients with locally advanced cervical cancer? A cohort control study using definitive chemoradiotherapy with high-dose rate brachytherapy
}

\author{
Shang-Wen Chen ${ }^{1,3,4}$, Ji-An Liang ${ }^{1,3}$, Yao-Ching Hung ${ }^{2,3}$, Lian-Shung Yeh ${ }^{2,3}$, \\ Wei-Chun Chang ${ }^{2,3}$, Wu-Chou Linn ${ }^{2,3}$, Chun-Ru Chien ${ }^{1,3}$ \\ ${ }^{1}$ Department of Radiation Therapy and Oncology, China Medical University Hospital, Taichung, Taiwan
2 Department of Obstetrics and Gynecology, China Medical University Hospital, Taichung, Taiwan
${ }^{3}$ College of Medicine, China Medical University, Taichung, Taiwan
${ }^{4}$ College of Medicine, Taipei Medical University, Taipei, Taiwan
}

Radiol Oncol 2013; 47(2): 176-184

Received 12 May 2012

Accepted 24 December 2012

Correspondence to: Shang-Wen Chen, Department of Radiation Oncology, China Medical University Hospital, No. 2, Yuh-Der Road, Taichung, Taiwan 404. Phone: +886-4-2205-2121, E-mail: vincent1680616@yahoo.com.tw

Disclosure: No potential conflicts of interest were disclosed.

Background. Comparing initial 45 Gy of pelvic intensity-modulated radiation therapy (IMRT) and non-IMRT in terms of the late toxicities associated with advanced cervical cancer that has also been treated with definitive concurrent chemoradiotherapy and high-dose rate intracavitary brachytherapy (HDRICB).

Patients and methods. This retrospective study included 320 stage IB2-IIIB cervical cancer patients treated with CCRT (83 IMRT and 237 non-IMRT). The two groups had similar stage and HDRICB ratings. Following 45 Gy to the pelvis, HDRICB of $24 \mathrm{~Gy}$ in four courses was prescribed. Late toxicities, including rectal complications (RC), bladder complications (BC) and non-rectal intestinal injury (NRRII), were scored by the Common Terminology Criteria for Adverse Events. A logistic regression was used to estimate the odds ratio (OR) of the complications.

Results. With a median follow-up duration of 33 and 77 months for IMRT and non-IMRT, 33 patients had Grade 2 or higher late RC (7.2\% IMRT, 1 1.4\% non-IMRT), whereas that for BC was 40 (9.6\% IMRT, 13.5\% non-IMRT) and for NRRIl was 48 (12.0\% IMRT, 16.0\% non-IMRT). The cumulative rate for total grade 3 or higher gastrointestinal or genitourinary toxicities was $8.4 \%$ and $11.8 \%$ ( $p=0.33$ ). IMRT did not reduce the OR for all endpoints; however, the ORs for rectum and bladder reference doses to Point A were associated with RC and BC.

Conclusions. Locally advanced cervical cancer patients treated with initial 45Gy of pelvic IMRT and HDRICB have similar treatment-related late toxicities as those treated with non-IMRT. Optimization of the brachytherapy scheme is essential to minimize late toxicities.

Key words: cervical cancer; IMRT; brachytherapy; complication

\section{Introduction}

The use of intensity-modulated radiation therapy (IMRT) for gynecological malignancies has grown considerably ${ }^{1}$, despite limited data on long-term toxicities and survival. Several studies have shown that IMRT reduces bowel, rectal, bladder, and bone marrow dose and is associated with lower rates of 
TABLE 1. Patient and tumor characteristics for the IMRT and non-IMRT groups

\begin{tabular}{|c|c|c|c|c|c|}
\hline \multirow{2}{*}{ Characteristicss } & \multicolumn{2}{|c|}{$\begin{array}{l}\text { IMRT } \\
\mathrm{N}=83\end{array}$} & \multicolumn{2}{|c|}{$\begin{array}{c}\text { Non-IMRT } \\
\mathrm{N}=237\end{array}$} & \multirow{2}{*}{$\begin{array}{c}p \\
\text { value }\end{array}$} \\
\hline & $\mathrm{n}$ & (\%) & $\mathrm{n}$ & (\%) & \\
\hline Median age (years) & \multicolumn{2}{|c|}{54} & \multicolumn{2}{|c|}{54} & $0.47^{*}$ \\
\hline Stage & & & & & 0.80 \\
\hline IB2-IIA2 & 20 & $(24.1)$ & 49 & (20.7) & \\
\hline$\| B$ & 38 & $(45.8)$ & 113 & $(47.7)$ & \\
\hline$\|I \mid A-\| B$ & 25 & $(30.1)$ & 75 & $(31.6)$ & \\
\hline Histology & & & & & 0.87 \\
\hline squamous cell ca. & 74 & $(89.2)$ & 214 & $(90.3)$ & \\
\hline adenocarcinoma or adenosquamous & 9 & $(10.8)$ & 23 & $(9.7)$ & \\
\hline Pelvic lymph node status & & & & & 0.83 \\
\hline negative & 59 & $(71.1)$ & 181 & (76.4) & \\
\hline positive & 24 & $(28.9)$ & 56 & (23.6) & \\
\hline Patients with brachytherapy number $=5$ & 12 & $(14.5)$ & 24 & $(10.1)$ & 0.75 \\
\hline Patients with reduced point A dose ( $\leq 5 \mathrm{~Gy}$ ) & 17 & $(20.5)$ & 33 & (13.9) & 0.37 \\
\hline Six or more courses of concurrent cisplatin & 58 & $(71.1)$ & 164 & (69.2) & 0.90 \\
\hline Diabetes & 8 & $(9.6)$ & 22 & $(9.2)$ & 0.88 \\
\hline Median follow-up (months) & \multicolumn{2}{|c|}{33 (range:13 54) } & \multicolumn{2}{|c|}{77 (range: 36 115) } & $0.000^{*}$ \\
\hline
\end{tabular}

*examined by t-test

early gastrointestinal, genitourinary, and hematological toxicity compared with conventional techniques. ${ }^{1-9}$ Furthermore, many IMRT studies have been associated with better outcomes. ${ }^{1-3}$ Although current findings suggest a favorable impact of IMRT on outcomes, so far, no randomized phase 3 trial has been conducted to examine its benefits probably due to ethical concerns. Despite IMRT became increasingly popular for gynecological cancer patients treated at many institutions, it remains unclear whether the lower rates of toxicity observed in our patients with an intact uterus. In this circumstance, brachytherapy might be an important factor affecting local control and late toxicities..$^{10}$ Particularly, concurrent cisplatin may also cause an increased incidence of hematological and gastrointestinal side effects. ${ }^{11}$ A large cohort study has compared the impact on patients of IMRT with patients treated by non-IMRT as a control cohort. ${ }^{2}$ They showed that pseudo-step-wedge intensity modulation can achieve better survival and less treatment-related late toxicities. However, currently this technique is not commonly used in most institutes. Furthermore, all the patient and treatment variables should be compared together before IMRT can be assumed to improve therapeutic ratio.

Previous studies have suggested that organ motion and deformation of the target volumes occur during IMRT for cervical cancer. ${ }^{12-16}$ Furthermore, a study showed a relative reduction in volume during treatment of $0.02-0.79 .{ }^{16}$ In consequence, adjacent normal organs might receive an unexpected irradiation dose following the shrinkage of the tumor during fractionated external beam radiotherapy (EBRT). The combination of unpredictable organ motion and substantial tumor regression has resulted in a consensus guideline suggestion that margins of 1.5 to $2 \mathrm{~cm}$ around clinical target volume (CTV) are to be recommended if good quality daily soft tissue verification was available during treatment. ${ }^{17}$ Thus, certain limitations exist when the physician try to reduce the physical dose affecting the adjacent normal tissues when using IMRT.

In definitive chemoradiotherapy for locally advanced cervical cancer, in which brachytherapy is still an essential component of radiotherapy, the net impact of pelvic IMRT on late toxicities remains to be determined. We conducted a retrospective cohort comparison study with controlled stage and demographic distributions in order to clarify the impact of IMRT on late complications. All the included patients were treated with the same pelvic EBRT dose of 45 Gy in 1.8 Gy daily fractions, concurrent chemotherapy and a standard brachytherapy scheme, namely high-dose rate intracavitary brachytherapy (HDRICB). Furthermore, several 


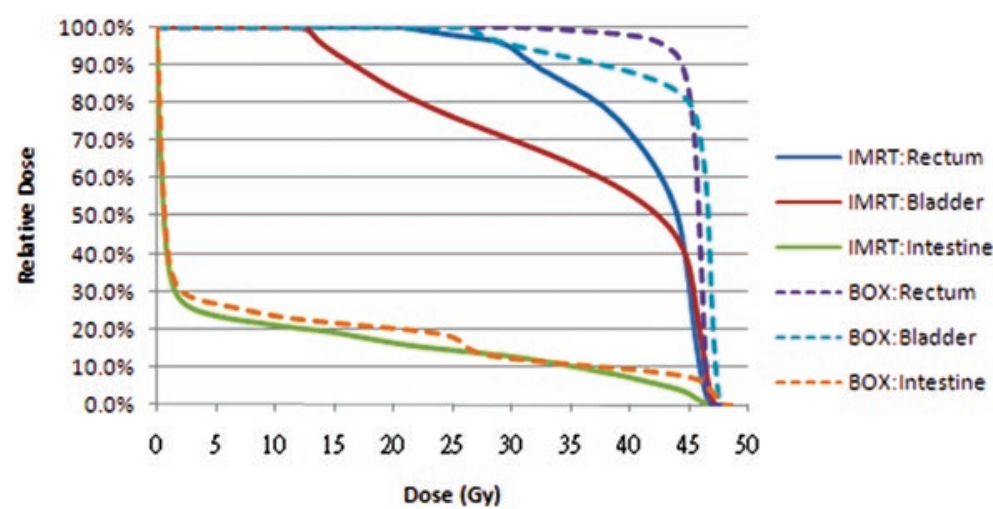

FIGURE 1. Dose-volume histiogram of adjacent normal organs between the IMRT and 4 -field box field for one patient.

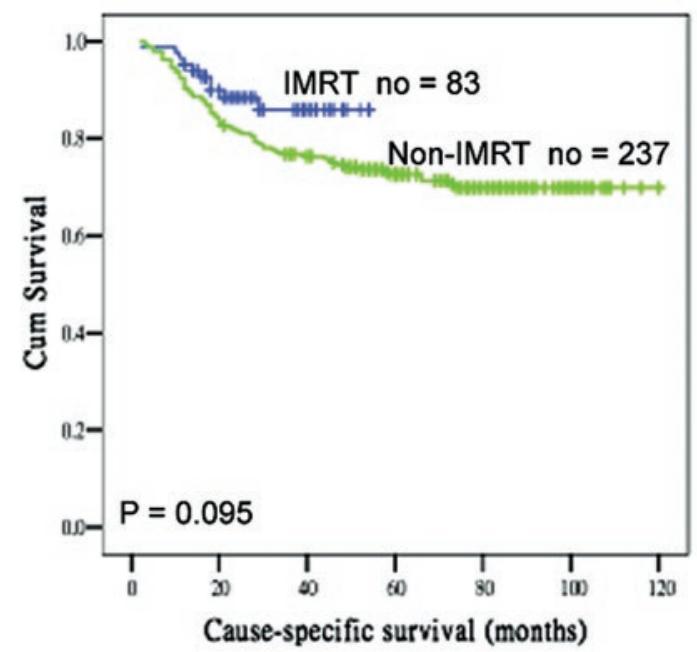

FIGURE 2. Cause-specific survival curves according to IMRT and non-IMRT groups.

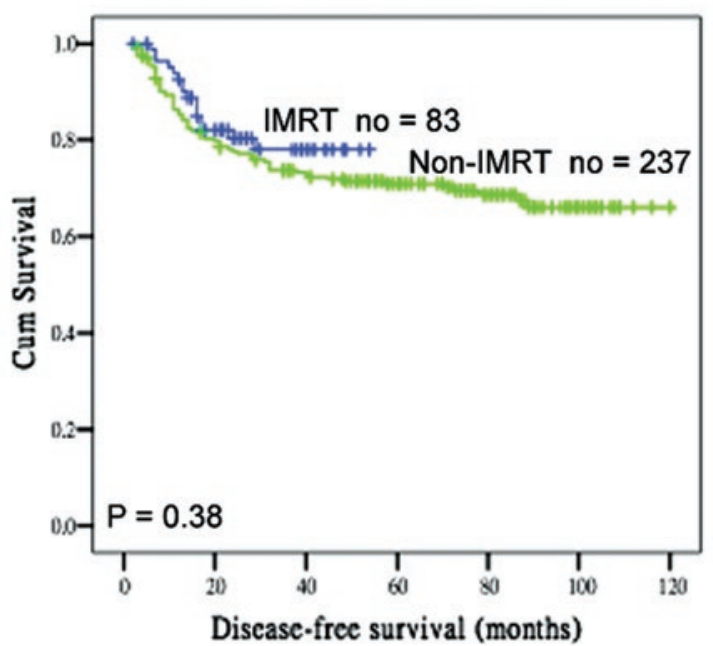

FIGURE 3. Disease-free survival curves according to IMRT and non-IMRT groups. patient and treatment variables were analyzed to assess the impact of IMRT. The result will be helpful to institutions where the cost-effectiveness of IMRT is a concern, particularly where resources are limited.

\section{Patients and Methods}

\section{Patients}

This retrospective cohort study included 320 patients with stage IB2-IIIB cervical cancer treated with curative intent (83 IMRT and 237 non-IMRT) between 2002 and 2009 at China Medical University Hospital. Since 2007, most patients with newly diagnosed cervical cancer have been prospectively treated with an IMRT to the pelvis, and are labeled as the IMRT group. All patients in the two groups received comprehensive pretreatment workup, including computed tomography, and completed an allocated CCRT course. Positron emission tomography (PET) was used for the workup in selected patients who were observed to have a maximum diameter of lymph node of more than $1 \mathrm{~cm}$. The two groups had similar stage and histological distributions. Patients with positive paraaortic lymph node, an extended field, or distant metastasis at diagnosis were excluded. The characteristics of the two groups are listed in Table 1. Except for the follow-up duration, all the patient-related or brachytherapy-related factors were similar. The study was approved by the institutional review board.

\section{Conventional external beam radiotherapy}

All patients underwent CT-based planning with custom immobilization. Initially, the whole pelvis was treated with $10 \mathrm{MV}$ X-ray via anterior and posterior parallel fields or box fields when the AP diameter was greater than $18 \mathrm{~cm}$. We prescribed an EBRT dose of 45 Gy in 25 fractions over 5 weeks to the whole pelvis. Generally, a field margin of at least $1.5 \mathrm{~cm}$ around the gross tumor was used, as described previously. ${ }^{18}$ Then, bilateral parametrial disease was boosted to 50.4 to $59.6 \mathrm{~Gy}$ via anterior and posterior parallel field technique with a rectangular central shielding of $4 \mathrm{~cm}$ width. By this technique, the mean doses to the rectum were kept less than $10 \%$ of the boost doses, whereas that for the bladder fewer than $40 \%$. Accordingly, we made the assumption that the major contributor of EBRT to the both organs would derive from the initial pelvic radiotherapy (RT). 
TABLE 2. Late complications between the IMRT and non-IMRT groups

\begin{tabular}{|c|c|c|c|c|c|c|}
\hline \multirow{2}{*}{ Classification of complication } & \multicolumn{2}{|c|}{$\begin{array}{l}\text { IMRT } \\
\mathrm{N}=83\end{array}$} & \multicolumn{2}{|c|}{$\begin{array}{c}\text { Non-IMRT } \\
\mathrm{N}=237\end{array}$} & \multirow{2}{*}{ total } & \multirow{2}{*}{$\begin{array}{c}p \\
\text { value }\end{array}$} \\
\hline & $\mathbf{n}$ & $(\%)$ & $\mathbf{n}$ & (\%) & & \\
\hline $\begin{array}{l}\text { Grade } 2 \text { or higher RC } \\
\text { Grade } 3 \text { or higher RC }\end{array}$ & $\begin{array}{l}6 \\
2\end{array}$ & $\begin{array}{l}(7.2) \\
(2.4)\end{array}$ & $\begin{array}{c}27 \\
6\end{array}$ & $\begin{array}{c}(11.4) \\
(2.5)\end{array}$ & $\begin{array}{c}33 \\
8\end{array}$ & $\begin{array}{l}0.24 \\
0.99\end{array}$ \\
\hline $\begin{array}{l}\text { Grade } 2 \text { or higher } B C \\
\text { Grade } 3 \text { or higher } B C\end{array}$ & $\begin{array}{l}8 \\
3\end{array}$ & $\begin{array}{l}(9.6) \\
(3.6)\end{array}$ & $\begin{array}{l}32 \\
14\end{array}$ & $\begin{array}{c}(13.5) \\
(5.9)\end{array}$ & $\begin{array}{l}40 \\
17\end{array}$ & $\begin{array}{l}0.25 \\
0.32\end{array}$ \\
\hline $\begin{array}{l}\text { Grade } 2 \text { or higher NRRII } \\
\text { Grade } 3 \text { or higher NRRII }\end{array}$ & $\begin{array}{c}10 \\
4\end{array}$ & $\begin{array}{c}(12.0) \\
(4.8)\end{array}$ & $\begin{array}{l}38 \\
16\end{array}$ & $\begin{array}{l}(16.0) \\
(6.7)\end{array}$ & $\begin{array}{l}48 \\
21\end{array}$ & $\begin{array}{l}0.38 \\
0.76\end{array}$ \\
\hline $\begin{array}{l}\text { Total grade } 2 \text { or higher gastrointestinal } \\
\text { or genitourinary complications }\end{array}$ & 19 & $(22.9)$ & 71 & $(30.0)$ & 90 & 0.24 \\
\hline $\begin{array}{l}\text { Total grade } 3 \text { or higher gastrointestinal } \\
\text { or genitourinary complications }\end{array}$ & 7 & $(8.4)$ & 28 & $(11.8)$ & 35 & 0.33 \\
\hline Grade 2 or higher leg edema & 9 & (10.8) & 16 & $(6.8)$ & 25 & 0.43 \\
\hline
\end{tabular}

$\mathrm{RC}=$ rectal complication; $\mathrm{BC}=$ bladder complication; NRR $\|$ = non-rectal intestinal injury.

\section{IMRT technique}

Intensity-modulated radiation therapy plans consisted of 7 coplanar fields using 10 MV photons. The prescription dose to the whole pelvis was 45 Gy. Following IMRT, bilateral parametrial disease was boosted to 50.4 to 59.6 Gy via anterior and posterior parallel fields with the same central shielding as mentioned above.

In the IMRT group, the CTV included the gross disease, cervix, parametrium, uterus, superior half of the vagina, cardinal ligament, presacral region, and regional lymph nodes (common, internal, and external iliac). Inguinal nodes were treated in women with involvement of the inferior third of the vagina. The CTV delineation was similar to the consensus guidelines on CTV delineation emerged. ${ }^{17}$ Uniform planning margins were added to account for organ motion and setup uncertainty. According to previous studies ${ }^{12,13}$, we applied a 15 $\mathrm{mm}$ planning margin around the cervix, a $10 \mathrm{~mm}$ margin around the uterus and the vagina, and a 8 $\mathrm{mm}$ margin around the remainder of the CTV.

Target planning constraints became standardized as follows: (1) more than $97 \%$ of the planning target volume (PTV) receives more than $97 \%$ of the prescription dose, (2) less than $1 \%$ of the PTV receives less than $93 \%$ of the prescription dose, (3) less than $5 \%$ of the PTV receives more than $107 \%$ of the prescription dose. When prescribing $45 \mathrm{~Gy}$ of dose to the whole pelvis, normal tissue planning constraints were consistent and were as follows: (1) rectum, less than $50 \%$ of volume receives greater than $45 \mathrm{~Gy}$; (2) bladder, less than $50 \%$ of volume receives greater than $45 \mathrm{~Gy}$, and (3) non-rectal bowel, less than $10 \%$ of volume receives greater than 45
Gy. No special constraint was used for the bone marrow. The constraints on dose-volume histogram (DVH) for a normal organ given above were not mandatory when the physician considered it necessary not to compromise the PTV coverage. Figure 1 depicts the DVH of adjacent normal organs between the IMRT and box field for one patient.

\section{Brachytherapy}

After adequate tumor regression, HDRICB was performed using an Ir-192 remote after-loading technique at 1 week intervals and concurrently with pelvic irradiation or parametrial boosting. The standard prescribed dose for each HDRICB was 6.0 Gy to Point A for four sessions. The Point A dose was reduced to 5.0 Gy for those with higher reference doses to the rectum or bladder, or whose age was over 70 years. The total prescribed Point A doses (EBRT + HDRICB) of a radiobiological equivalent dose in 2 Gy fractions $\left(\mathrm{EQD}_{2}\right.$ ) ranged from 69.25 to 84.25 (median, 76.25). The details of the radiotherapy technique have been reported previously. ${ }^{19}$

The geometric sparing factor (GSF) is defined as the average of the ratios between the reference dose and the Point A dose during each HDRICB insertion. The mean GSF for the rectum (abbreviated as RGSF) = the average of the ICRU rectal dose/ Point A dose. The mean GSF for the bladder (abbreviated as BGSF) $=$ the average of the ICRU bladder dose/Point A dose. The description of GSF was described in our previous study..$^{19}$ Accordingly, in this study the RGSFs and BGSFs were stratified with cuff-offs of 0.7 and 0.9 , respectively. 
TABLE 3. Multivariate logistic regression estimated odds ratios (OR) for developing grade 2 or higher RC across different variables

\begin{tabular}{lccc}
\hline Variables & $\begin{array}{c}p \\
\text { value }\end{array}$ & OR & $95 \%$ Cl \\
\hline Age $<65$ vs. $\geq 65$ years & 0.12 & 1.34 & $0.84 \sim 2.82$ \\
Age $<70$ vs. $\geq 70$ years & 0.43 & 1.12 & $0.38 \sim 2.29$ \\
Diabetes & 0.70 & 1.32 & $0.39 \sim 8.31$ \\
negative vs. positive & 0.93 & 1.03 & $0.52 \sim 2.07$ \\
Stage IB2-IIA vs. IIB-IIIB & 0.44 & 0.68 & $0.26 \sim 1.77$ \\
Non-IMRT vs. IMRT & 0.67 & 0.76 & $0.22 \sim 2.56$ \\
Parametrial dose $\geq 54$ vs. > 54 Gy & 0.065 & 3.49 & $0.93 \sim 13.17$ \\
RAL-IC number 4 vs. 5 & 0.40 & 1.64 & $0.51 \sim 5.25$ \\
RGSF $<0.7$ vs. $\geq 0.7$ & 0.003 & 3.36 & $1.52 \sim 7.43$ \\
$\geq$ Grade 2 BC & 0.000 & 2.18 & $1.44 \sim 3.30$ \\
\hline
\end{tabular}

$\mathrm{RC}=$ rectal complication; $\mathrm{BC}=$ bladder complication; $\mathrm{NRRII}=$ non-rectal intestinal injury; $\mathrm{RGSF}=$ geometric sparing factor of the rectum; $\mathrm{BGSF}=$ geometric sparing factor of the bladder.

\section{Chemotherapy}

Chemotherapy consisted of cisplatin delivered weekly at a dose of $40 \mathrm{mg} / \mathrm{m}^{2}$ intravenously, with a total maximal dose of up to $60 \mathrm{mg}$. The first cycle of cisplatin was initiated at the first RT treatment. In accordance with the duration of RT, the treatment plan included a total of five to six cycles of cisplatin. The detailed drug administration protocol was described in our previous study. ${ }^{18}$

\section{Follow-up}

After completion of radiotherapy, patients received regular follow-up every 1 to 2 months for the first year, and then every 3 months afterward. A pelvic examination was performed during each follow-up; in addition, tumor markers (squamous cell carcinoma antigen and carcinoembryonic antigen) were checked. A radiographic examination was carried out every 3 to 6 months and routine urine and stool examinations were done every 6 to 12 months. Patients who had persistent cramping abdominal pain, bloody stools or hematuria underwent sigmoidoscopy or cystoscopy to identify the source of bleeding, and underwent blood counts every 2 to 4 weeks for surveillance of the severity of the complications.

\section{Complication analysis}

Common Terminology Criteria for Adverse Events Version 3.0 was used to score the maximum late toxicities, including rectal complications (RC), bladder complications (BC), non-rectal intestinal injury (NRRII), and leg edema. The definition of the NRRII was reported in our previous study. ${ }^{20}$ There were several study endpoints, including grade 2 and higher RC, grade 2 and higher $\mathrm{BC}$, grade 2 and higher NRRII, total grade 2 and higher gastrointestinal or genitourinary complications, and total grade 3 and higher gastrointestinal or genitourinary complications.

\section{Statistics}

A comparison of the categorical variables was performed using the $\chi^{2}$ test. A Student's $t$ test was used to compare differences in continuous variables when patients were stratified into the two groups. A logistic regression was used to estimate the odds ratio (OR) of complications among the variables examined. Although we believe that a longer follow-up duration is needed to estimate survival differences between the two groups, cause-specific survival (CSS) and disease-free survival (DFS) were calculated using the Kaplan-Meier method to provide preliminary results for the two treatment regimes. Statistical significance was considered to have occurred when a two-sided $p$ value of $<0.05$ was found. Patient survival was measured from the date of radiotherapy initiation to the last followup. The latency of complications was measured from the end of radiotherapy to the last followup. All statistical analyses were performed using a commercial software package (SPSS 13.0 for Windows, Chicago, IL, USA). 
TABLE 4. Multivariate logistic regression estimated odds ratios (OR) for developing grade 2 or higher BC across different variables

\begin{tabular}{lccc}
\hline Variables & $\begin{array}{c}p \\
\text { value }\end{array}$ & OR & $95 \%$ Cl \\
\hline Age $<65$ vs. $\geq 65$ years & 0.57 & 0.69 & $0.18 \sim 2.56$ \\
Age $<70$ vs. $\geq 70$ years & 0.66 & 1.43 & $0.32 \sim 6.40$ \\
Diabetes $\quad$ negative vs. positive & 0.47 & 1.61 & $0.43 \sim 8.52$ \\
Stage IB2-IIA vs. IIB-IIIB & 0.66 & 1.13 & $0.65 \sim 1.97$ \\
Stage IB2-IIB vs. IIIA-IIIB & 0.36 & 0.68 & $0.29 \sim 1.57$ \\
Non-IMRT vs. IMRT & 0.10 & 0.42 & $0.15 \sim 1.19$ \\
Parametrial dose $\geq 54$ vs. $>54$ Gy & 0.57 & 0.78 & $0.29 \sim 2.01$ \\
RAL-IC number 4 vs. 5 & 0.055 & 2.39 & $0.98 \sim 5.82$ \\
BGSF $<0.9$ vs. $\geq 0.9$ & 0.04 & 2.01 & $1.01 \sim 4.32$ \\
$\geq$ Grade 2 RC & 0.000 & 2.47 & $1.65 \sim 3.70$ \\
\hline
\end{tabular}

$\mathrm{RC}=$ rectal complication; $\mathrm{BC}=$ bladder complication; NRRII = non-rectal intestinal injury; $\mathrm{RGSF}=$ geometric sparing factor of the rectum; $\mathrm{BGSF}=$ geometric sparing factor of the bladder.

\section{Results}

The mean follow-up duration for the 320 patients enrolled at the time of last visit was 62 months (33 months IMRT, 77 months non-IMRT). At the time of last follow-up, 78 patients died of cancer, 10 in the IMRT group, and 68 in the non-IMRT group. Eighty-eight patients had developed a recurrence, 16 in the IMRT group, and 72 in the non-IMRT group. There was a similar pattern of recurrences between the two groups, with more than $80 \%$ of patients having distant recurrences. The 3 years CSS for the IMRT and non-IMRT groups were $86 \%$ and $76 \%(p=0.095)$, whereas the 3 years DFS was $78 \%$ and $74 \%(p=0.37)$ (Figure 1$)$.

Of the patients, 33 patients had Grade 2 or higher late RC (6 IMRT, 27 non-IMRT). In all, 40 patients had Grade 2 or higher late BC (8 IMRT, 32 non-IMRT), whereas 48 patients had Grade 2 or higher NRRII (10 IMRT, 38 non-IMRT). The median time for the development of RC, BC and NRRII was 12 months (range, 7-35 months), 19 months (range, 3-49 months) and 13 months (range, 3-28 months). The cumulative rate for total grade 2 or higher gastrointestinal or genitourinary complications was $22.9 \%(19 / 83)$ in the IMRT group and $30.0 \%(71 / 237)$ in the non-IMRT group $(p=0.24)$, whereas that for total grade 3 or higher complications was $8.4 \%(7 / 83)$ among IMRT patients and $11.8 \%(28 / 237)$ among non-IMRT patients $(p=$ 0.33). Among IMRT patients, the cumulative rate for grade 3 or higher RC, BC and NRRII were $2.4 \%$,
$3.6 \%$ and $4.8 \%$, respectively. Details of the various complication endpoints for the IMRT and non-IMRT patients are listed in Table 2.

The correlation of patient and treatment related factors with several complication endpoints are summarized in Tables 3 to 5. Logistic regression analysis demonstrated a high risk of Grade 2 and higher $\mathrm{RC}$ in patients who developed $\mathrm{BC}$ complications ( $p=0.000$; odd ratio [OR], 2.18, 95\% confidence interval $[\mathrm{CI}], 1.44-3.30)$ and in those with higher RGSF values $(p=0.003$; OR, 3.36; 95\% CI, 1.52-7.43). Furthermore, there was a high risk of Grade 2 or higher BC in patients who developed RC ( $p=0.000$; OR, 2.47, 95\% CI, 1.65-3.70) and in those with a higher BGSF values $(p=0.04$; OR, 2.01; 95\% CI, 1.01-4.32). There were high risk factors for Grade 2 or higher NRIII among those patients having $\geq$ grade 2 RC ( $p=0.001 ; \mathrm{OR}, 1.99 ; 95 \% \mathrm{CI}, 1.26-$ 3.16). In general, IMRT was associated with a lower risk of developing most late toxicities; however, the trend was not statistically significant. Age, clinical stage, diabetes, parametrial dose and ICB number were not associated with increased risk of late sequelae by multivariate analysis.

To minimize the confounding impact of the GSFs on toxicities analysis, patients were further stratified according to lower and higher $50 \%$ percentile of the GSFs for rectum and bladder. As showed in Table 6, there was no significant difference between IMRT and non-IMRT groups for developing grade 2 or greater complications when they were categorized according to the median GSF values. 
TABLE 5. Multivariate logistic regression estimated odds ratios (OR) for developing grade 2 or higher NRRll across different variables

\begin{tabular}{lccc}
\hline Variables & $\begin{array}{c}p \\
\text { value }\end{array}$ & OR & $95 \% \mathrm{Cl}$ \\
\hline Age $<65$ vs. $\geq 65$ years & 0.29 & 0.46 & $0.11 \sim 1.94$ \\
Age $<70$ vs. $\geq 70$ years & 0.70 & 1.38 & $0.27 \sim 7.10$ \\
Diabetes & 0.96 & 1.25 & $0.23 \sim 8.95$ \\
$\quad$ negative vs. positive & 0.47 & 1.20 & $0.72 \sim 2.00$ \\
Stage IB2-IIA vs. IIB-IIIB & 0.34 & 1.45 & $0.68 \sim 3.09$ \\
Stage IB2-IIB vs. IIIA-IIIB & 0.33 & 0.64 & $0.26 \sim 1.58$ \\
Non-IMRT vs. IMRT & 0.31 & $0.63 \sim 1.54$ \\
Parametrial dose $\leq 54$ vs. > 54 Gy & 0.34 & 0.59 & $0.20 \sim 1.76$ \\
RAL-IC number 4 vs. 5 & 0.42 & 2.14 & $0.33 \sim 13.75$ \\
RGSF $<0.7$ vs. $\geq 0.7$ & 0.68 & 0.72 & $0.15 \sim 3.41$ \\
BGSF $<0.9$ vs. $\geq 0.9$ & 0.001 & 1.99 & $1.26 \sim 3.16$ \\
$\geq$ Grade 2 RC & 0.74 & 1.08 & $0.68 \sim 1.73$ \\
\hline Grade 2 BC & & 0.25 \\
\hline
\end{tabular}

$\mathrm{RC}=$ rectal complication; $\mathrm{BC}=$ bladder complication; $\mathrm{NRRII}=$ non-rectal intestinal injury; $\mathrm{RGSF}=$ geometric sparing factor of the rectum; $\mathrm{BGSF}=$ geometric sparing factor of the bladder.

\section{Discussions}

The application of IMRT is believed to result in the organs at risk being exposed to a lower dose of radiation and consequently there will be a reduction in toxicities. Currently, long-term comparison data remain limited in the setting of definitive CCRT for locally advanced cervical cancer. Taking into consideration that the follow-up duration in this study was not very long (median, 33 months), we found that initial 45 Gy of IMRT to the pelvis did not reduce significantly the long-term complications as reported in other comparable studies., ${ }^{2,3}$ By analyzing several complication endpoints, not simply classify the late toxicities as gastrointestinal and genitourinary system, or solely reporting grade 3 late toxicities, this study comprehensively explored the CCRT-related toxicities during definitive treatment for cervical cancer. Unlike those treated in the postoperative setting, in which irradiation dose is mainly prescribed by external beam, brachytherapy always plays an important role in determining the final outcome for patients with an intact uterus. Thus, the application of IMRT ought to be one of the determinants of outcome. The impact of external beam and brachytherapy should be assessed together due to the cumulative effect of both modalities.

With the longest median follow-up duration among other similar IMRT studies ${ }^{1-3,9}$, we report here that the cumulative rates for grade 3 or higher RC, BC and NRRII to be $2.4 \%, 3.6 \%$ and $4.8 \%$.
Furthermore, our study disclosed that total grade 3 or greater toxicities among patients were $8.4 \%$. Despite the significant variation in follow-up duration, the figure seems to be comparable with the other studies..$^{1-3,9}$ In a study by Hasselle et al. ${ }^{1}$, the rate of grade 3 and greater complications in 89 patients with intact cervix treated with IMRT plus low-dose rate was $4 \%$ and $5 \%$ for gastrointestinal and genitourinary system, respectively. Particularly, Kidd et al. ${ }^{2}$, showed a great difference in the incidence of late toxicities between the groups. Their IMRT group had only a $6 \%$ rate of Grade 3 or greater GI or GU toxicity, versus 17\% for the non-IMRT group ( $p=0.0017)$, whereas the median follow-up duration for IMRT and non-IMRT was 22 and 72 months, respectively. Despite the majority of the urinary and rectal complications occurring within 2 to 3 years after the completion of therapy, the risk of developing grade 3 late complications might occur up to 25 years after treatment, as pointed out by Eifel et al. ${ }^{21}$ Thus, a long-term observational study is essential to verify the findings of the recent IMRT studies.

This study showed that RGSF and BGSF were treatment-related factors in determining grade 2 or greater $\mathrm{RC}$ and $\mathrm{BC}$, respectively. In addition, a close relationship between the two complications was observed. All these findings verify the results of our previous study. ${ }^{19}$ Thus, an optimization of the HDRICB is essential to minimizing late complications. Although our current treatment scheme (45 Gy to whole pelvis plus 24 Gy of HDRICB Point 
TABLE 6. Patients with or without IMRT on having grade 2 or higher complications according to lower and higher $50 \%$ percentile of geometric sparing factor for rectum and bladder

\begin{tabular}{|c|c|c|c|c|}
\hline Groups & Complication & IMRT (\%) & non-IMRT (\%) & $\begin{array}{c}p \\
\text { value* }\end{array}$ \\
\hline $\begin{array}{l}\text { Lower RGSF group } \\
\text { (RGSF <0.635) } \\
\text { no }=156\end{array}$ & $\begin{array}{l}R C(+) \\
R C(-)\end{array}$ & $\begin{array}{c}1(2.2 \%) \\
43(97.8 \%)\end{array}$ & $\begin{array}{c}6(5.4 \%) \\
106(94.6 \%)\end{array}$ & 0.88 \\
\hline $\begin{array}{l}\text { Higher RGSF group } \\
\text { (RGSF }>0.635) \\
\text { no }=155\end{array}$ & $\begin{array}{l}R C(+) \\
R C(-)\end{array}$ & $\begin{array}{c}5(13.2 \%) \\
33(86.8 \%)\end{array}$ & $\begin{array}{c}16(13.7 \%) \\
101(86.3 \%)\end{array}$ & 0.75 \\
\hline $\begin{array}{l}\text { Lower BGSF group } \\
\text { (BGSF < } 0.695) \\
\text { no }=157\end{array}$ & $\begin{array}{l}\mathrm{BC}(+) \\
\mathrm{BC}(-)\end{array}$ & $\begin{array}{c}3(7.0 \%) \\
40(93.0 \%)\end{array}$ & $\begin{array}{l}17(14.9 \%) \\
97(85.1 \%)\end{array}$ & 0.30 \\
\hline $\begin{array}{l}\text { Higher BGSF group } \\
(\text { BGSF }>0.695) \\
\text { no }=158\end{array}$ & $\begin{array}{l}B C(+) \\
B C(-)\end{array}$ & $\begin{array}{r}5(12.5 \%) \\
35(87.5 \%)\end{array}$ & $\begin{array}{c}18(15.3 \%) \\
100(84.7 \%)\end{array}$ & 0.97 \\
\hline
\end{tabular}

Note: *examined by Chi-square test

$\mathrm{RC}=$ rectal complication; $\mathrm{BC}=$ bladder complication; NRRII = non-rectal intestinal injury; $\mathrm{RGSF}=$ geometric sparing factor of the rectum; $\mathrm{BGSF}=$ geometric sparing factor of the bladder.

A divided into four courses) is able to achieve a similar outcome compared with other IMRT studies $^{1-3,9}$, many aspects remains to be improved. First, it is imperative to continue efforts to explore genetic predisposition in order to determine which patients are susceptible to radiation-induced normal tissues injury. Second, image-based HDRICB studies are appropriate approaches that might be used to minimize complications further. ${ }^{22}$ In this context, despite the fact that our study classified late gastrointestinal toxicities into RC and NRRII, the irradiated intestinal DVH due to each brachytherapy remains unknown. This is also a major drawback when investigating the NRRII. In the future, image-based brachytherapy might be used to calculate the intestinal volume during each application. By counting the intestinal DVH due to IMRT and brachytherapy separately and together, a comprehensive dosimetric analysis for NRRII could be obtained.

The results should be interpreted with several limitations. When comparing outcomes using a historical control, the follow-up duration between groups is always a weakness when presuming the final treatment outcome. Although IMRT patients had a shorter follow-up duration, the result showed a trend towards similar complications between the groups. Accordingly, we assumed the irradiated strategy of combining IMRT with HDRICB needs to be optimized further. Second, despite most volumes of the rectum and bladder were spared during the parametrial boost field, the IMRT benefit could be somewhat diluted by the non-IMRT boost technique. To optimize the preferable dose distribution made by IMRT, our study highlighted a need to develop a special IMRT boost technique to exactly match the isodose of brachytherapy. Finally, larger margins in the pelvic IMRT tend to diminish the degree of organ sparing; the advantage of the dose distribution from IMRT should be intensified by the general implementation of image-guided RT and adaptive RT to circumvent interfraction or intrafraction motion. By correcting the uncertainty after tumor regression, the adaptive approaches allow a reduction in the margin that is added to the CTV. In this way, the therapeutic index ought to be promoted. Nonetheless, by investigating several complication endpoints via an analysis of many patient and treatment factors, our experience will be helpful to those institutions where HDRICB is performed. In the future, monitoring the information of quality of life before and after CCRT would be essential to clarify the benefit of IMRT. ${ }^{23}$ Although we failed to demonstrate the robust advantage of IMRT in definitive CCRT for advanced cervical cancer, the result will also help where the cost-effectiveness of IMRT is a concern, particularly when the resources are limited.

\section{Conclusions}

Locally advanced cervical cancer patients treated with a combination of $45 \mathrm{~Gy}$ of pelvic IMRT and HDRICB have similar treatment-related late toxicities compared with those treated with a similar non-IMRT regimen. The ratios of rectum and bladder reference doses to Point $A$ are associated with $\mathrm{RC}$ and BC. For those institutions where HDRICB is performed, optimization of the combining IMRT 
and brachytherapy scheme is essential to minimize late toxicities.

\section{Acknowledgements}

We want to thank the grant support from Taiwan Department of Health, Cancer Research Centers for Excellence (DOH101-TD-C-111-005).

\section{References}

1. Hasselle MD, Rose BS, Kochanski JD, Nath SK, Bafana R, Yashar CM, et al. Clinical outcome of intensity-modulated pelvic radiation therapy for carcinoma of the cervix. Int J Radiat Oncol Biol Phys 2011; 80: 1436-45.

2. Kidd EA, Siegel BA, Dehdashti F, Rader JS, Mutic S, Mutch DG, et al. Clinical outcomes of definitive intensity-modulated radiation therapy with fluorodeoxyglucose-positron emission tomography simulation in patients with locally advanced cervical cancer. Int J Radiat Oncol Biol Phys 2010; 77: 1085-91.

3. Mundt AJ, Mell LK, Roeske JC. Preliminary analysis of chronic gastrointestinal toxicity in gynecology patients treated with intensity-modulated whole pelvic radiation therapy. Int J Radiat Oncol Biol Phys 2003; 56: 1354-60.

4. Ferrigno R, Santos A, Martins LC, Weltman E, Chen MJ, Sakuraba R, et al. Comparison of conformal and intensity modulated radiation therapy techniques for treatment of pelvic tumors. Analysis of acute toxicity. Radiat Oncol 2010; 5: 117

5. Brixey CJ, Roeske JC, Lujan AE, Yamada SD, Rotmensch J, Mundt AJ. Impact of intensity-modulated radiotherapy on acute hematologic toxicity in women with gynecologic malignancies. Int J Radiat Oncol Biol Phys 2002; 54: 1388-96

6. Chen MF, Tseng CJ, Tseng CC, Kuo YC, Yu CY, Chen WC. Clinical outcome in posthysterectomy cervical cancer patients treated with concurrent cisplatin and intensity-modulated pelvic radiotherapy: Comparison with conventional radiotherapy. Int J Radiat Oncol Biol Phys 2007; 67: 1438-44.

7. Roeske JC, Bonta D, Mell LK, Lujan AE, Mundt AJ. A dosimetric analysis of acute gastrointestinal toxicity in women receiving intensitymodulated whole-pelvic radiation therapy. Radiother Oncol 2003; 69: 201-7.

8. Mell LK, Kochanski JD, Roeske JC, Haslam JJ, Mehta N, Yamada SD, et al. Dosimetric predictors of acute hematologic toxicity in cervical cance patients treated with concurrent cisplatin and intensity-modulated pelvic radiotherapy. Int J Radiat Oncol Biol Phys 2006; 66: 1356-65.

9. Chen CC, Lin JC, Jan JS, Ho SC, Wang L. Definitive intensity-modulated radiation therapy with concurrent chemotherapy for patients with locally advanced cervical cancer. Gynecol Oncol 2011; 122: 9-13.

10. Rogelj P, Hudej R, Petric P. Distance deviation measure of contouring variability. Radiol Oncol 2013; 47: 86-96.

11. Keys HM, Bundy BN, Stehman FB, Muderspach LI, Chafe WE, Suggs CL, et al. Cisplatin, radiation, and adjuvant hysterectomy compared with radiation and adjuvant hysterectomy for bulky stage IB cervical carcinoma. $N$ Engl J Med 1999; 340: 1154-61.

12. Taylor A, Rockall AG, Reznek RH, Powell ME. Mapping pelvic lymph nodes: Guidelines for delineation in intensitymodulated radiotherapy. Int J Radiat Oncol Biol Phys 2005; 63: 1604-12.

13. Lim K, Chan P, Dinniwell R, Fyles A, Haider M, Cho YB, et al. Cervical cance regression measured using weekly magnetic resonance imaging during fractionated radiotherapy: Radiobiologic modeling and correlation with tumor hypoxia. Int J Radiat Oncol Biol Phys 2008; 70: 126-33.

14. van de Bunt L, Jürgenliemk-Schulz IM, de Kort GA, Roesink JM, Terstee $\mathrm{RJ}$, van der Heide UA. Motion and deformation of the target volumes during IMRT for cervical cancer: What margins do we need? Radiother Oncol 2008; 88: 233-40.
15. Chan P, Dinniwell R, Haider MA, Cho YB, Jaffray D, Lockwood G, et al. Interand intrafractional tumor and organ movement in patients with cervica cancer undergoing radiotherapy: A cinematic-MRI point-of interest study. Int J Radiat Oncol Biol Phys 2008; 70: 1507-15.

16. Beadle BM, Jhingran A, Salehpour M, Sam M, Iyer RB, Eifel PJ. Cervix regression and motion during the course of external beam chemoradiation for cervical cancer. Int J Radiat Oncol Biol Phys 2009; 73: 235-41.

17. Lim K, Small W, Portelance L, et al. Consensus guidelines for delineation of clinical target volume for intensity-modulated pelvic radiatherapy for the definitive treatment of cervix cancer. Int J Radiat Oncol Biol Phys 2011; 79: 348-55.

18. Chen SW, Liang JA, Hung YC, Yeh LS, Chang WC, Lin WC, et al. Concurrent weekly cisplatin plus external beam radiotherapy and high-dose rate brachytherapy for advanced cervical cancer: a control cohort comparison with radiation alone on treatment outcome and complications. Int J Rad Oncol Biol Phys 2006; 66: 1370-77.

19. Chen SW, Liang JA, Hung YC, Yeh LS, Chang WC, Lin WC, et al. Geometrica sparing factors for the rectum and bladder in the prediction of grade 2 and higher complications following high-dose-rate brachytherapy for cervical cancer. Int J Rad Oncol Biol Phys 2009; 75: 1335-43.

20. Chen SW, Liang JA, Yang SN, Hung YC, Yeh LS, Shiau AC, et al. Radiation injury to intestine following hysterectomy and adjuvant radiotherapy for cervical cancer. Gynecol Oncol 2004; 95: 208-14.

21. Eifel PJ, Levenback C, Wharton JT, Oswald MJ. Time course and incidence of late complications in patients treated with radiation therapy for FIGO Stage IB carcinoma of the uterine cervix. Int J Rad Oncol Biol Phys 1995; 32: $1289-300$.

22. Potter R, Haie-Meder C, Van Limbergen E, Barillot I, De Brabandere $M$ Dimopoulos J, et al. Recommendations from gynaecological GEC ESTRO working group (II): Concepts and terms in 3D image-based treatment planning in cervical cancer brachytherapy-3D dose volume parameters and aspects of 3D image-based anatomy, radiation physics, radiobiology. Radiother Oncol 2006; 78: 67-77.

23. Ljuca D, Marosevic G. Quality of life in patients with cervical cancer FIGO IIb stage after concomitant chemoradiotherapy. Radiol Oncol 2009; 43: 293-8. 